\title{
Proteasomal ATPases Hard at Work: The Inner Workings of a Protein Destruction Machine.
}

Aaron M. Snoberger

Follow this and additional works at: https://researchrepository.wvu.edu/etd

\section{Recommended Citation}

Snoberger, Aaron M., "Proteasomal ATPases Hard at Work: The Inner Workings of a Protein Destruction Machine." (2018). Graduate Theses, Dissertations, and Problem Reports. 8186.

https://researchrepository.wvu.edu/etd/8186

This Dissertation is protected by copyright and/or related rights. It has been brought to you by the The Research Repository @ WVU with permission from the rights-holder(s). You are free to use this Dissertation in any way that is permitted by the copyright and related rights legislation that applies to your use. For other uses you must obtain permission from the rights-holder(s) directly, unless additional rights are indicated by a Creative Commons license in the record and/ or on the work itself. This Dissertation has been accepted for inclusion in WVU Graduate Theses, Dissertations, and Problem Reports collection by an authorized administrator of The Research Repository @ WVU.

For more information, please contact researchrepository@mail.wvu.edu. 


\title{
Proteasomal ATPases Hard at Work: The Inner Workings of a Protein Destruction Machine
}

\author{
Aaron M. Snoberger
}

\author{
Dissertation submitted \\ to the School of Medicine \\ at West Virginia University \\ in partial fulfillment of the requirements for the degree of \\ Doctor of Philosophy in \\ Biochemistry and Molecular Biology
}

\author{
David M. Smith, Ph.D., Chair \\ Michael D. Schaller, Ph.D. \\ Maxim Sokolov, Ph.D. \\ Michael R. Gunther, Ph.D. \\ David P. Siderovski, Ph.D.
}

Department of Biochemistry

Morgantown, West Virginia

2018

Keywords: proteasome, molecular motor, Rpt, ATPase, AAA, AAA+ Copyright 2018 Aaron M. Snoberger 


\section{Abstract \\ Proteasomal ATPases Hard at Work: The Inner Workings of a Protein Destruction Machine Aaron M. Snoberger}

Across all domains of life, the proteasome is responsible for the majority of targeted protein degradation in the cell. Often, the proteasome is thought of as the molecular "garbageman" of the cell. While it is true that the proteasome degrades and eliminates misfolded proteins, the proteasome is also capable of degrading fully folded, functional proteins whose presence is no longer required (e.g. during embryonic development, cell cycle changes, etc.). Despite its crucial role in virtually every cellular process, our understanding of how the proteasome operates from a mechanistic perspective is still highly limited. In order to prevent unregulated degradation the protease sites of the proteasome are sequestered inside its hollow interior. While loosely folded proteins can enter the degradation chamber without the requirement of energy, proteins with secondary structure can only be degraded when they are properly recognized (e.g. by ubiquitin tags), unfolded, and injected into the protease chamber for degradation. Protein recognition, unfolding, and injection into the protease chamber all depend on ATP. However, very little is known about how such chemical energy is converted to mechanical work. In this dissertation we sought to understand the logistics of nucleotide binding and hydrolysis, and also to determine the conformational changes that can regulate protein entry and degradation by the proteasome.

To this end, we focused on one of the most common regulators from eukaryotes-- the heterohexameric 19S ATPases, as well as its homohexameric archaeal homolog-- "PAN" (proteasome activating nucleotidase). Based on our extensive analysis, our data support a neighbor-binding sequential hydrolysis mechanism for the proteasomal ATPases. Furthermore, we show that these ATPases are highly processive, even when they reach more tightly folded domains of a protein, which is unlike what had been proposed previously based on studies of other ATP-dependent proteases (e.g. ClpX, which often "slips" and "stalls" at these more tightly folded domains). This tight binding of the proteasomal ATPases appears to be due to its crucial trans-arginine fingers that "sense" bound nucleotides in its neighboring subunit (which ClpX lacks), and we propose that this processivity arose due to the diverse client proteins the proteasome must encounter (e.g. it must engage and unfold many types of proteins, even ones that are fully folded and functional).

Lastly, we have developed a disulfide engineering approach to show that PAN's N-terminal domains adopt distinct conformations that set the rate of ATP hydrolysis. This novel approach has allowed us to isolate specific subunits from a homohexamer that are identical in their amino acid sequence, but that adopt different conformations when they form a hexameric ring. This disulfide engineering approach we've developed is a powerful method to analyze structural asymmetries in homomeric protein complexes with minimal structural perturbations, which has not been accomplished before and opens the door to an entire new approach to studying the function of the molecular motors.

We started this work with the goal of understanding the logistics of the mechano-chemical cycle of the proteasomal ATPases. Indeed, we have developed novel methods to better understand the inner workings of this complex multimeric machine, and the groundwork we lay here has contributed greatly to our knowledge of the proteasomal ATPases, and will also push forward our understanding of other AAA+ ATPases and molecular motors in general. Ultimately, a better understanding of these complex machines will aid in the development of new therapies to combat diseases where these machines are dysregulated. 


\section{Dedication}

"It's easier to steer a moving ship"

To my family and friends who encouraged me to keep moving, even in those times that I didn't know what I was moving toward. It's always easier to change course if you don't stagnate, a philosophy that's proven just as relevant in the 'science world' as it has in the 'real world'.

To Jess, my best friend, there's no other co-pilot l'd rather share this crazy journey with. 


\section{Acknowledgments}

Thanks to my graduate mentor, David Smith, for guiding me through this process. You've given me the freedom to pursue projects I'm passionate about, and your mentoring style has helped me to grow and be independent in ways that many don't experience until their post-docs. I couldn't have asked for a better mentor-- you've been patient enough to let me learn from my mistakes, yet have always been there to lend help when I need it. My wife and I are also grateful for how you lead by example-you always work hard, yet always find time for what is most important in life. You've truly been a great mentor in every sense of the word.

Thanks to Rob Wysolmerski, my undergraduate research advisor, for introducing me to the fascinating world of biomedical research. Thanks to my committee members, Michael Schaller, Max Sokolov, Michael Gunther, Dave Siderovski, and former member Bill Wonderlin for guiding my research. Thanks to the members of the "old guys" journal club for challenging me and helping to develop my critical reading of the literature.

Thanks to Evan Brettrager for substantially increasing my productivity in your time here-with your help I had the time to pursue the more risky projects that turned out to be quite fruitful, and thanks to Taylor Thomas for carrying on this exciting research after my departure. Lastly, thanks to present and past members of the Smith Lab for making this journey an enjoyable one: Jane Schupp, Tiffany Thibaudeau, Young-Chan Kim, Evan Brettrager, Ray Anderson, and Taylor Thomas. 


\section{Table of Contents}

Title Page

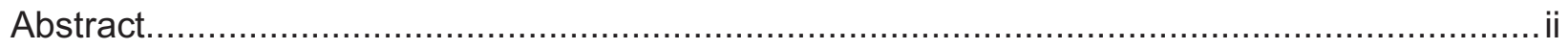

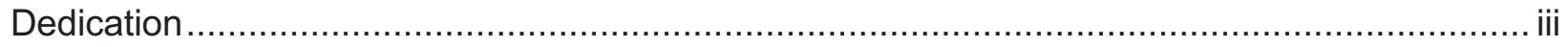

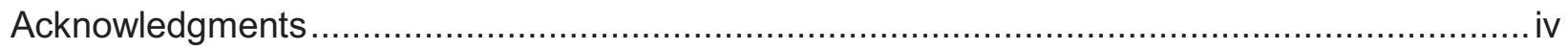

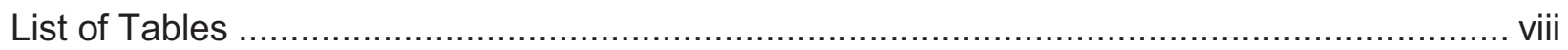

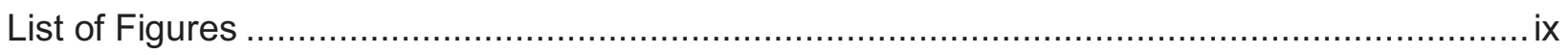

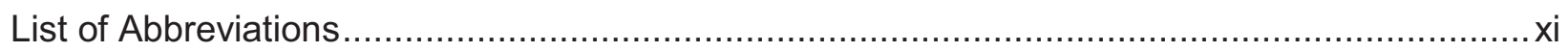

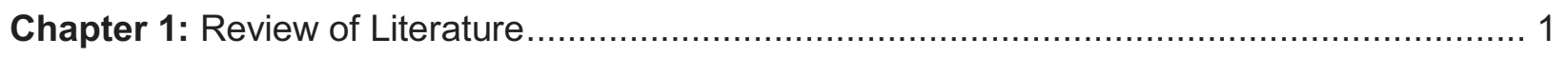

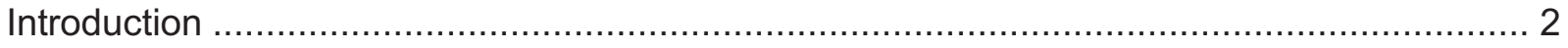

Proteasomal ATPases from other species are closely related to the 19S ............................. 4

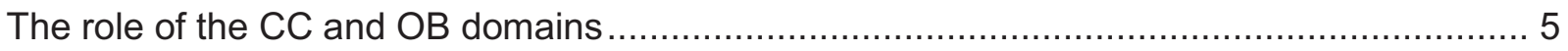

The role of the AAA+ ATPase domain in proteasomal ATPases.......................................... 5

How do substrates stimulate their own degradation: A role for the $\mathrm{CC}$ domains? ................... 8

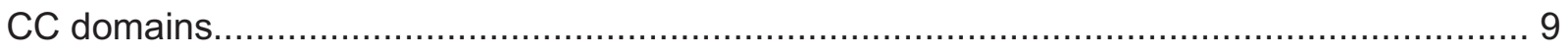

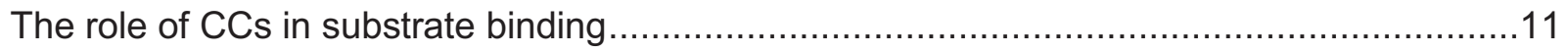

The role of CCs in chaperone activity and ATPase activity ..........................................13

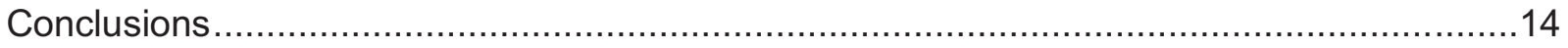

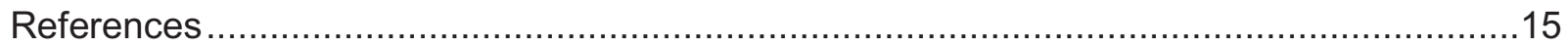

Chapter 2: ATP Binding to neighboring subunits and intersubunit coupling underlie proteasomal

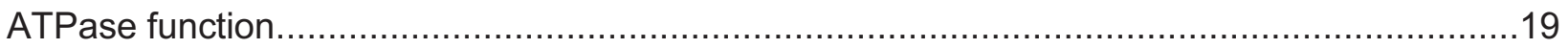

Preface

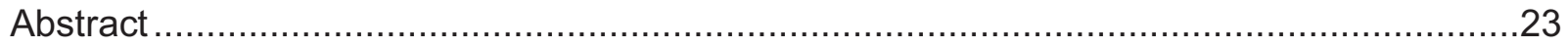

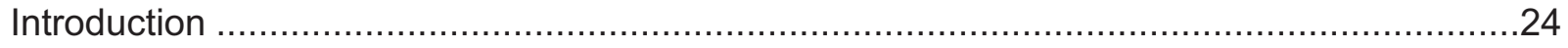

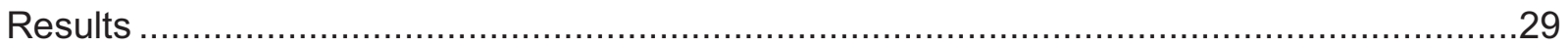

ATP binds to neighboring subunits ("Ortho" pattern) in the proteasomal ATPases .............29

Selection of the conserved arginine residues and generation of mutants .........................34

Both conserved arginine residues are required for ATP hydrolysis but not ATP-binding ....35

Neither stoichiometry nor binding pattern mutation of the conserved arginines ..................37 
ATP-binding cooperativity in PAN does not require the conserved arginine residues .........39

The conserved arginines are required for ATP-dependent substrate binding ..................41

The conserved arginines are required for ATPase-20S association and 20S gate opening43

The conserved arginines do not play a role in the dissociation kinetics of ADP

ATP-binding is in equilibrium during normal ATP hydrolysis- the ATP off rate is similar to

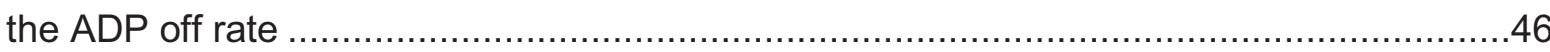

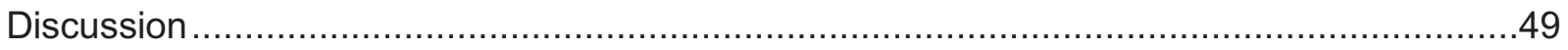

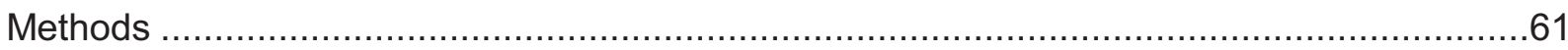

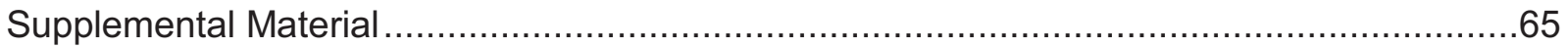

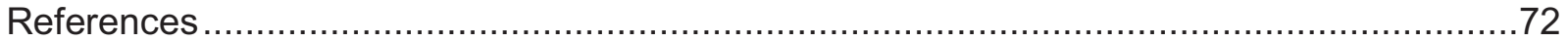

Chapter 3: The Proteasomal ATPases use a slow but highly processive strategy to unfold

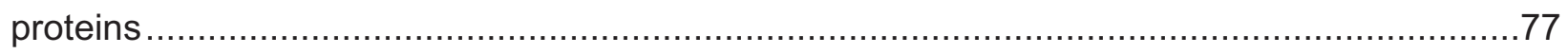

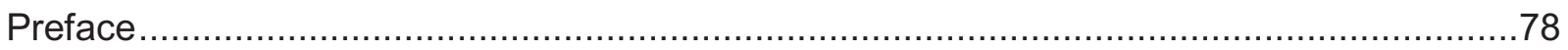

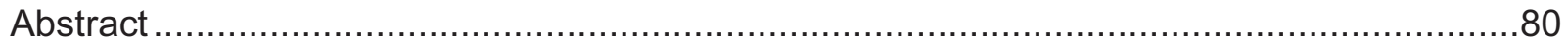

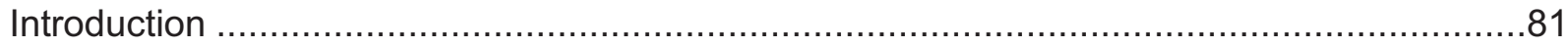

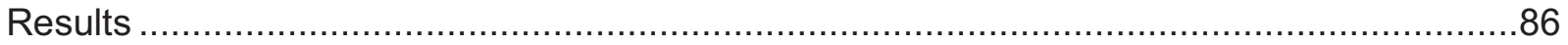

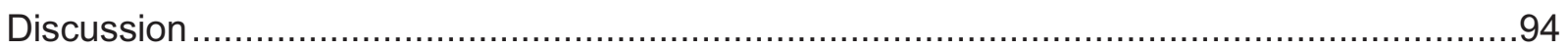

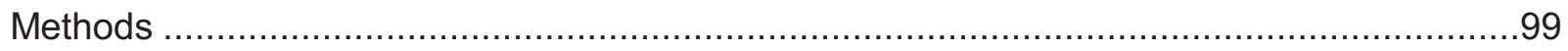

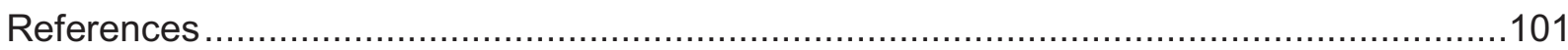

Chapter 4: Conformational switching in the coiled-coil domains of a proteasomal ATPase

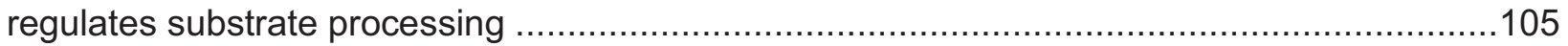

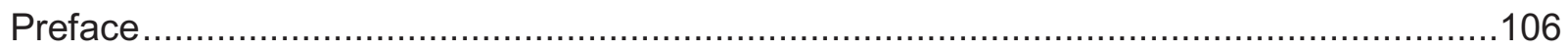

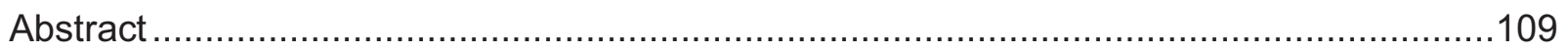

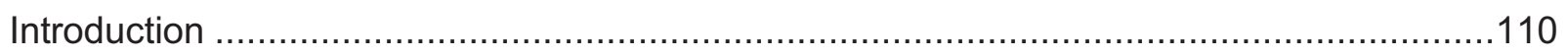

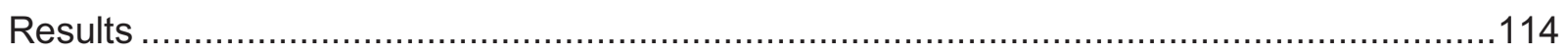

Only 1 of PAN's 3 Coiled-Coils is in the Expected Conformation ..................................114

Nucleotides Have Little Effect on Coiled-Coil conformation ........................................121

PAN's Coiled-Coils Adopt Distinct Conformations ................................................122

Coiled-Coil Conformations Regulate ATPase Rate \& Substrate Processing ..................128

PAN's Coiled-Coils Toggle Between "Activated" and "Resting" Conformations ................132

Discussion 


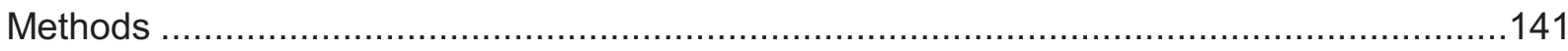

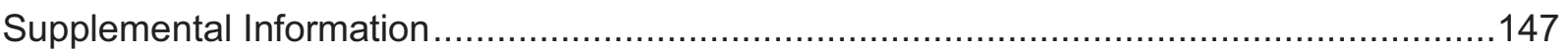

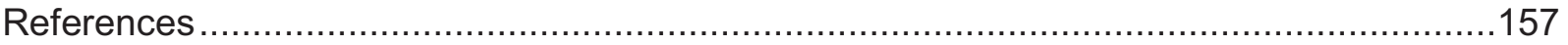

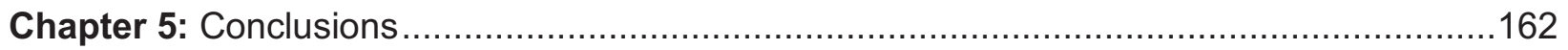

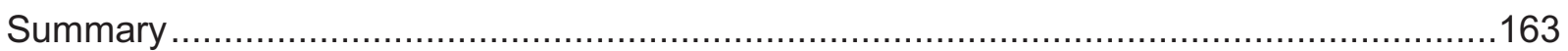

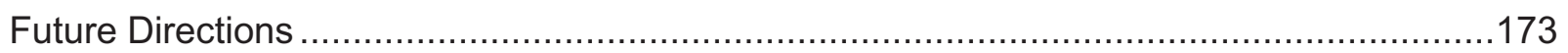

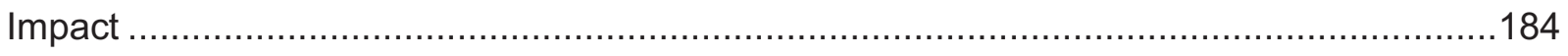

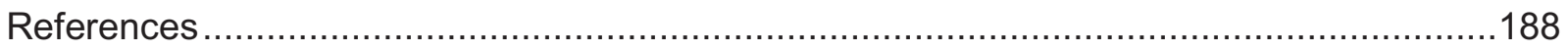




\section{List of Tables}

Table 2.1: FRET distance calculations between nucleotides

Table S4.1: Summary of ATP hydrolysis kinetics of PAN variants under oxidizing and reducing conditions

Table S4.2: Normalized ATP hydrolysis of PAN variants 


\section{List of Figures}

Figure 1.1: ATP-dependent protein degradation by the proteasome

Figure 1.2: Nucleotide binding exchange model for the proteasomal ATPases

Figure 1.3: The basic structure of dimeric, right handed coiled-coil domains

Figure 2.1: ATP binds to neighboring subunits ("Ortho" pattern) in the proteasomal ATPases.

Figure 2.2: Both of the conserved arginines in PAN are required for ATP hydrolysis but not for ATP binding.

Figure 2.3: PAN's conserved arginines are not involved in regulating ATP-binding affinity

Figure 2.4: PAN's conserved arginines are not involved in regulating nucleotide stoichiometry, or ATP-binding pattern.

Figure 2.5: Mutation of either one of PAN's conserved arginines abrogates ATPdependent substrate binding and $20 \mathrm{~S}$ gate-opening.

Figure 2.6: The ATP and ADP off rates are similar, and the ADP off rate is not affected by mutation of the arginine finger.

Figure 2.7: Allosteric model of nucleotide binding and exchange in the proteasomal ATPases and its implications on the trans-mechanism of substrate translocation.

Figure S2.1: Spectra of FRET pair

Figure S2.2: Structural model of the 26S ATPases and estimated distances between nucleotide binding sites.

Figure S2.3: ATP binding to PAN-E271Q is biphasic and fluorescent modifications have little effect on ATP affinity

Figure S2.4: Conserved arginine residues in the proteasomal ATPases and generation of arginine mutants in PAN

Figure S2.5: PAN's conserved arginines are not required for substrate unfolding

Figure S2.6. Careful purification of PAN arginine mutants to eliminate a highly active contaminating ATPase

Figure 3.1: Hexameric ATP-dependent proteases utilize energy from ATP hydrolysis to unfold substrates

Figure 3.2: PAN does not stall when it encounters the unfolded domain of GFP 
Figure 3.3: The eukaryotic $26 \mathrm{~S}$ does not stall when it encounters the folded domain of GFP

Figure 3.4: Comparison of the unfolding kinetics for the Proteasomal ATPases vs. ClpX

Figure 4.1: PAN contains 3 right handed, dimeric coiled-coils on its $\mathrm{N}$-terminus

Figure 4.2: PAN's coiled-coils do not adopt symmetrical conformations

Figure 4.3: PAN's ATPase domains induce a conformational asymmetry in its coiledcoils

Figure 4.4: One of PAN's three coiled-coils is partially unzipped

Figure 4.5: One of PAN's coiled-coils is 2 heptads out-of-register

Figure 4.6: Some coiled-coil conformations regulate PAN's activity

Figure 4.7: PAN adopts 2 distinct states: One that is fully active and another that is resting

Figure S4.1: PAN Mutants Retain Global Quaternary Structure and T20S Gate Opening Capacity

Figure S4.2: SDS-PAGE of the "F2" PAN-M87C fragment

Figure S4.3: "Natural" levels of nucleotides have little effect on disulfide crosslinking of WT PAN or PAN-M87C

Figure S4.4: PAN does not adopt a partial heptad slide

Figure S4.5: ATP hydrolysis kinetics of PAN mutants under oxidizing and reducing conditions

Figure S4.6: Asymmetric coiled-coil conformations are also observed in the $26 \mathrm{~S}$ ATPases

Figure S4.7: WT PAN dose response on SDS-PAGE

Figure 5.1: General logic to determine which subunits bind to nucleotides, substrates, or the 20S proteasome.

Fig 5.2: Determining the topology that the PAN hexamer adopts. 


\section{List of Abbreviations}
AAA+: ATPases Associated with Diverse Cellular Activities
ADP: Adenosine Diphosphate
ATP: Adenosine Triphosphate
CC: $\quad$ Coiled-Coil
CP: $\quad$ Core Particle
CTP: $\quad$ Cytidine Triphosphate
DNP: 2,4-Dinitrophenol
DTT: Dithiothreitol
EM: $\quad$ Electron Microscopy
FP: $\quad$ Fluorescence Polarization
FRET: Fluorescence Resonance Energy Transfer
GFP: Green Fluorescent Protein
GTP: Guanosine Triphosphate
ITP: Inosine Triphosphate
LDH: Lactate Dehydrogenase
LFP: $\quad$ Long Fluorescent Peptide
MCA: 7-Methoxycoumarin
Mpa: $\quad$ Mycobacterium Proteasomal ATPase
NADH: Nicotine Adenine Dinucleotide
NEM: N-ethylmaleimide
OB: Oligonucleotide/oligosaccharide binding
PAN: Proteasome Activating Nucleotidase
PEP: Phosphoenolpyruvate
PK: $\quad$ Pyruvate Kinase
PTM: Post-translational Modification
Pup: Prokaryotic Ubiquitin-like Protein
RP: $\quad$ Regulatory Particle
Rpn: Regulatory Particle non-ATPase Subunit
Rpt: $\quad$ Regulatory Particle ATP Hydrolyzing Subunit
SDS: Sodium Dodecyl Sulfate
SRH: Second Region of Homology
TNP: $\quad$ 2,4,6-Trinitrophenol
TT: $\quad$ Tetrathionate
TTP: $\quad$ Thymidine Triphosphate
Ub: Ubiquitin
UPS: Ubiquitin-Proteasome System
UTP: $\quad$ Uridine Triphosphate
WT: Wild-Type 
Chapter 1: Review of Literature 


\section{Introduction}

Appropriate protein synthesis and degradation are essential for virtually every cellular process, including cell cycle regulation, inflammation, antigen presentation, and degradation of short-lived or misfolded proteins (Gorbea et al. 2000). The ubiquitin proteasome system (UPS) is the primary mechanism for targeted protein degradation, and thus improper regulation of the UPS can contribute to a wide array of diseases (e.g. cancers, neurodegenerative diseases, cardiovascular diseases) (Jankowska et al. 2013). In the UPS, a substrate is tagged for degradation, often by polyubiquitin, and the $26 \mathrm{~S}$ proteasome recognizes this tag and degrades the protein into polypeptide fragments of $\sim 2-20$ amino acids (Fig. 1.1A). Ubiquitin is not degraded by the proteasome but rather is cleaved off by deubiquitinases on the $26 \mathrm{~S}$ prior to degradation of the substrate, and thus ubiquitin is recycled. The $26 \mathrm{~S}$ is comprised of 2 subcomplexes, the $19 \mathrm{~S}$ and the 20S. The $19 \mathrm{~S}$ caps one or both ends of the $20 \mathrm{~S}$ and acts to regulate protein entry into the $20 \mathrm{~S}$ (Fig. 1.1B). The $20 \mathrm{~S}$ is a 4-ringed, hollow barrel-like structure with a subunit topology of $\alpha-\beta-\beta-\alpha .3$ of the $7 \beta$ subunits in each ring contain catalytic sites sequestered on the inside of the chamber, while the $\alpha$ rings form 2 small central pores that are "gated" by their N-termini (Fig. 1.1B). Since the central pores at the a subunit interface are too small to allow for the entry of folded substrates, substrates must either be intrinsically disordered or be unfolded in order to enter the degradation chamber of the 20S, and can only enter when the 20S's gate is open. The $19 S$ is comprised of a heterohexameric ring of ATPase subunits (Rpt1-6) as well as 13 non-ATPase (Rpn) subunits (Fig. 1.1B). The Rpn subunits' main functions are to recognize substrate proteins and deubiquitinate them prior to entry into the $20 \mathrm{~S}$, while 

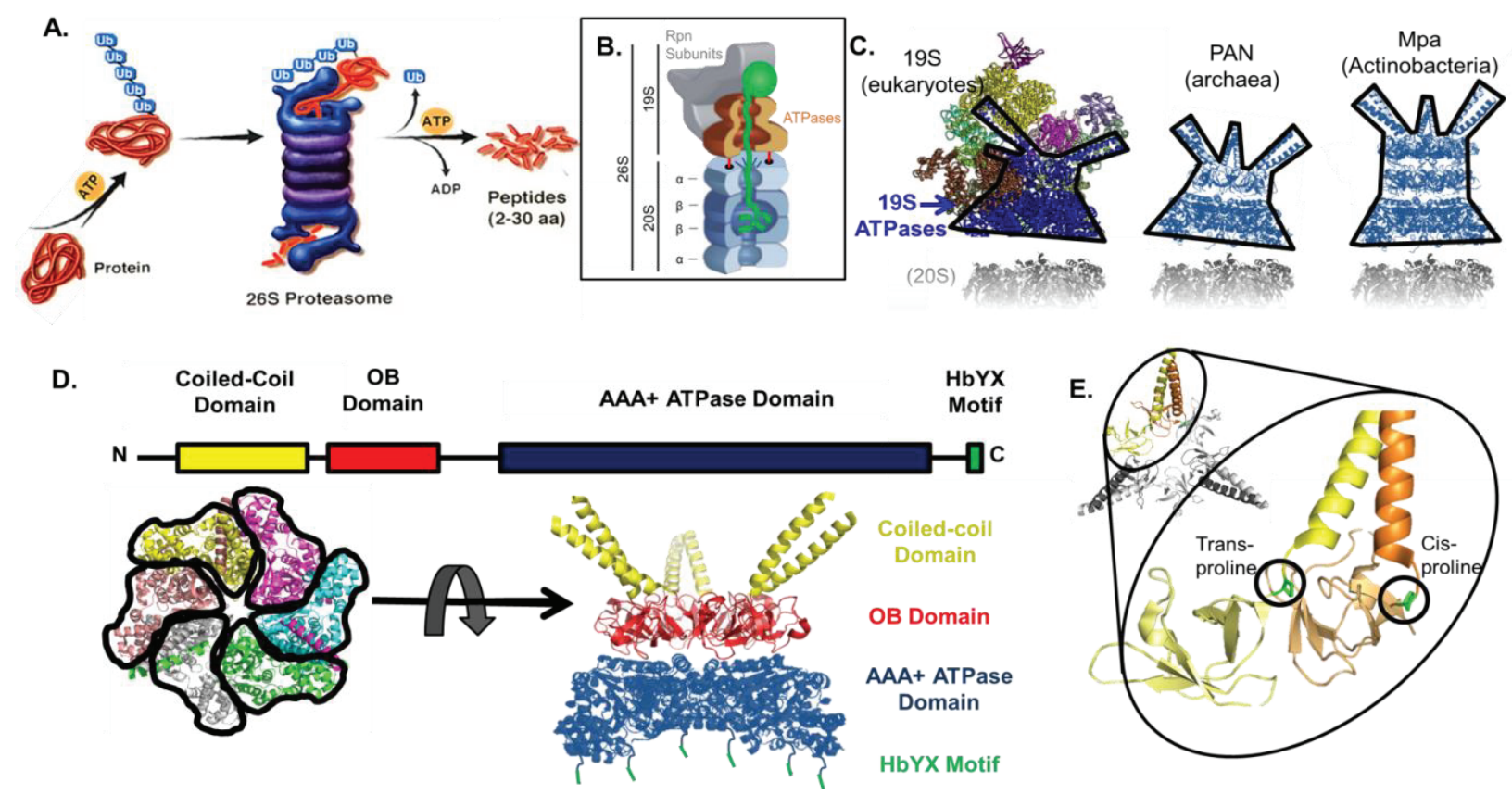

Figure 1.1: ATP-dependent protein degradation by the proteasome. A. A protein is tagged for degradation, often by polyubiquitin. This protein is recognized by the $26 \mathrm{~S}$ proteasome and the ubiquitin chain is cleaved while the protein is degraded into peptides of approximately 2-30 amino acids in length. B. The $26 \mathrm{~S}$ proteasome is made up of 2 subcomplexes, the $19 \mathrm{~S}$ and the 20S. The $20 \mathrm{~S}$ is made of 4 stacked rings arranged in an $\alpha-\beta-\beta-\alpha$ topology. The inside $\beta$-subunits have catalytic sites sequestered in the hollow, barrel-like structure of the 20S, while the outside $\alpha$ subunits have a central pore with a "gate" (dark blue). The 19S is made up of a ring of ATPase subunits and non-ATPase (Rpn) subunits. The C-terminal tail of the $19 \mathrm{~S}$ (red) insert into pockets of the $\alpha$ subunits and function to open the gate of the 20S. Unfolding and injection of substrates into the $20 \mathrm{~S}$ degradation chamber is accomplished by the ATPases. C. Comparison of PAN and Mpa to the ATPases of the 19S (outlined in black). D. Proteasomal ATPases form a hexameric ring and are composed of an N-terminal coiled-coil (CC) domain, followed by 1 or 2 oligonucleotide/ oligosaccharide binding (OB) domains, and a AAA+ ATPase domain with a C-terminal $\mathrm{HbYX}$ motif (hydrophobic, tyrosine, and any terminal amino acid). E. Crystal structure of the CC-OB domain of PAN. Alpha helices from adjacent subunits dimerize, and the hexamer is formed from a trimer of dimers. In PAN, cisand trans prolines (green) are responsible for kinking one alpha helix back toward the adjacent helix. 
the Rpt ring uses the energy from ATP hydrolysis to generate force that results in the unfolding of their substrate and their injection into the $20 \mathrm{~S}$ degradation chamber. The Ctermini of the ATPase subunits interact with intersubunit pockets on the $\alpha$-ring of the $20 S$ and change the free energy landscape of the pore by rotating the a subunits causing their $\mathrm{N}$-termini to move away from the pore, which effectively opens the gate of the 20 S proteasome for substrate entry (Yu et al. 2010; Fig. 1.1B).

\section{Proteasomal ATPases from other species are closely related to the $19 \mathrm{~S}$}

The closest known homolog to the eukaryotic 19S ATPase ring is the archaeal "proteasome activiating nucleotidase" (PAN) (Zwickl et al. 1999; Fig. 1.1C). Like the 19S ATPase it belongs to the AAA+ family of ATPases and is composed of an Nterminal coiled-coil (CC) domain, followed by an oligonucleotide/oligosaccharide binding (OB) domain, an AAA+ ATPase domain, and a c-terminal HbYX (hydrophobic, tyrosine, any terminal amino acid) motif (Zhang et al. 2009; Smith et al. 2007; Fig. 1.1D). There is also a related proteasome regulatory particle in actinobacteria called "mycobacterium proteasome ATPase" (Mpa) (Fig. 1.1C, right). The eukaryotic 19S ATPase is a heterohexamer while both PAN and Mpa are homohexamers. The 19S and PAN have 1 OB domain in each subunit, while Mpa has 2 copies of the OB domain in each subunit (Djuranovic et al. 2009). Since not a lot is known about the molecular functions of the 19S, this review will compile what is known about the 19S, PAN, and Mpa in an attempt to better understand the structure and function of proteasomal ATPases. 


\section{The role of the $\mathrm{CC}$ and $\mathrm{OB}$ domains}

Both the $\mathrm{CC}$ and $\mathrm{OB}$ domains are protein folds known to bind proteins, and based on their location above the substrate entry channel of the proteasomal ATPases they have been proposed to play a role in substrate binding (Rechsteiner et al. 1993). This Nterminal CC-OB domain is conserved across all domains of life (19S, PAN, and Mpa in eukaryotes, archaea, and mycobacteria, respectively), however, to date very little is known about its function. The only function that has been definitively shown for CC domains (at least in vitro) is the prevention the aggregation of proteins (i.e. chaperoning), presumably to prevent aggregation due to exposure of the substrate's hydrophobic residues during unfolding (Benaroudj \& Goldberg 2000). The specific sequence of the CC domains doesn't seem to matter for efficient chaperone activity, however, the sequence did seem to determine specificity for certain substrates (Djuranovic et al. 2009). In addition to chaperone activity, the CC-OB domain is also thought to mediate assembly of the hexameric complex through tight and stable interactions between dimers of the ring. In addition, cis-prolines in alternating subunits at the CC-OB interface allow its alpha helix to kink backwards in every other subunit to form a CC with the adjacent subunit's alpha helix (Fig. 1.1E). These dimers next come together to form a trimer of dimers (forming the complete hexamer), and the OB domain is thought to be critical for this interaction (Kish-Trier \& Hill 2013).

\section{The role of the AAA+ ATPase domain in proteasomal ATPases}

The AAA+ ATPase domain is responsible for binding and hydrolysis of nucleotides, which ultimately drives the functions of proteasomal ATPases (i.e. opening of the $20 \mathrm{~S}$ 
gate by the HbYX motif, substrate binding, substrate unfolding, substrate translocation into the 20S) (Smith et al. 2011). Unfolding and translocation of substrates is thought to be carried out by pore-loops, which are thought to interact with substrates and coordinated through axial movements that inject them through the pore of the ATPase and into the 20S, which also results in unfolding of the substrate due to forced threading through the pore of the OB-domain (Martin et al. 2008; Sen et al. 2013). In vitro, PAN can utilize both ATP and CTP (Wilson et al. 2000), though ATP binding and hydrolysis is likely the most physiologically relevant. The AAA+ ATPase domain is a hexamer and contains several conserved motifs involved in nucleotide binding and hydrolysis: including Walker A motif, Walker B motif, Sensor 1 motif, and arginine fingers (lyer et al. 2004). The 19S ATPase takes on a lockwasher-like conformation (Unverdorben et al. 2014), however, it is not known whether PAN also assumed such a conformation. Before the start of this dissertation work we determined that PAN had 2 high affinity and 2 low affinity sites for ATP while 2 subunits are unbound by nucleotide (Smith et al. 2011). We assumed that the high-affinity sites were ATP-bound and the low affinity sites were ADP-bound. Initially, we also envisioned that nucleotides bound symmetrically to "para" subunits, meaning that ATPs would bind to subunits across the ring (e.g. $180^{\circ}$ ) (Fig. 1.2A-B). Prior studies of similar ATP-dependent proteases (e.g. ClpX; Martin et al. Nature 2005) indicated that ATP hydrolysis occurred in a clockwise-manner, but this was not known for the proteasomal ATPases (Fig. 1.2B). However, as will become clear in Chapter 2 of this dissertation, our initial assumptions of a "para" binding pattern were incorrect, and that, in fact, proteasomal ATPases (both PAN and the 19S) bind to ATPs on neighboring subunits (e.g. an "ortho" binding pattern). Furthermore, we also 


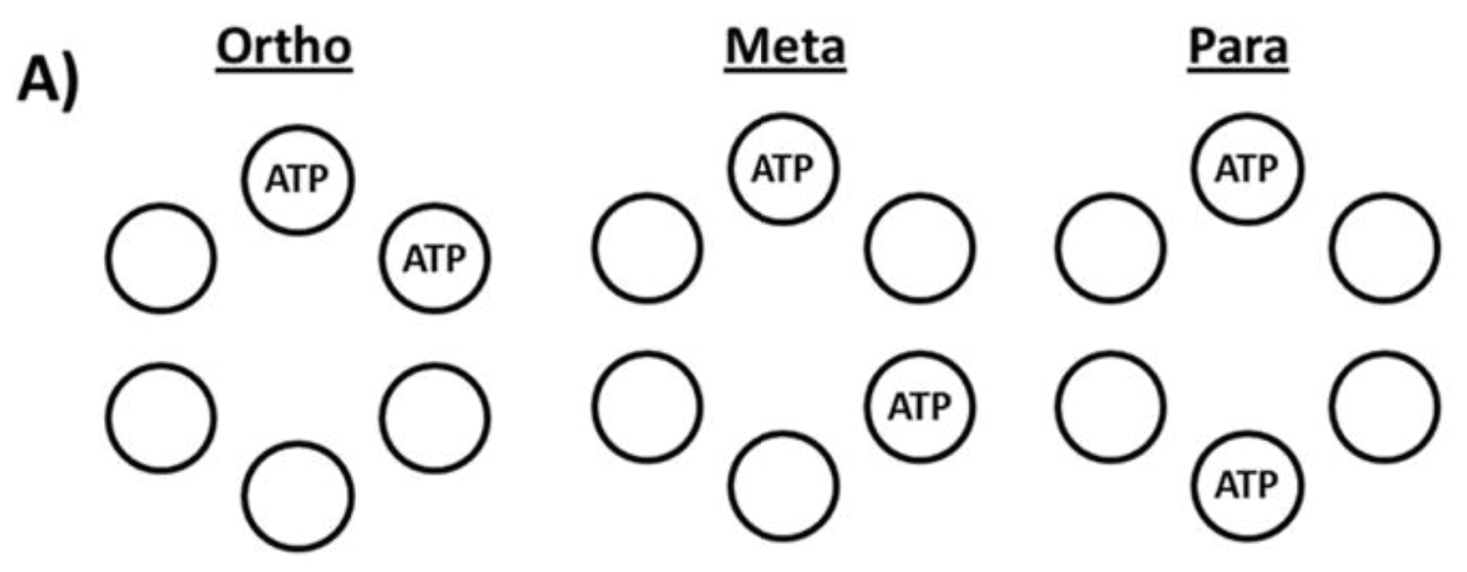

B)

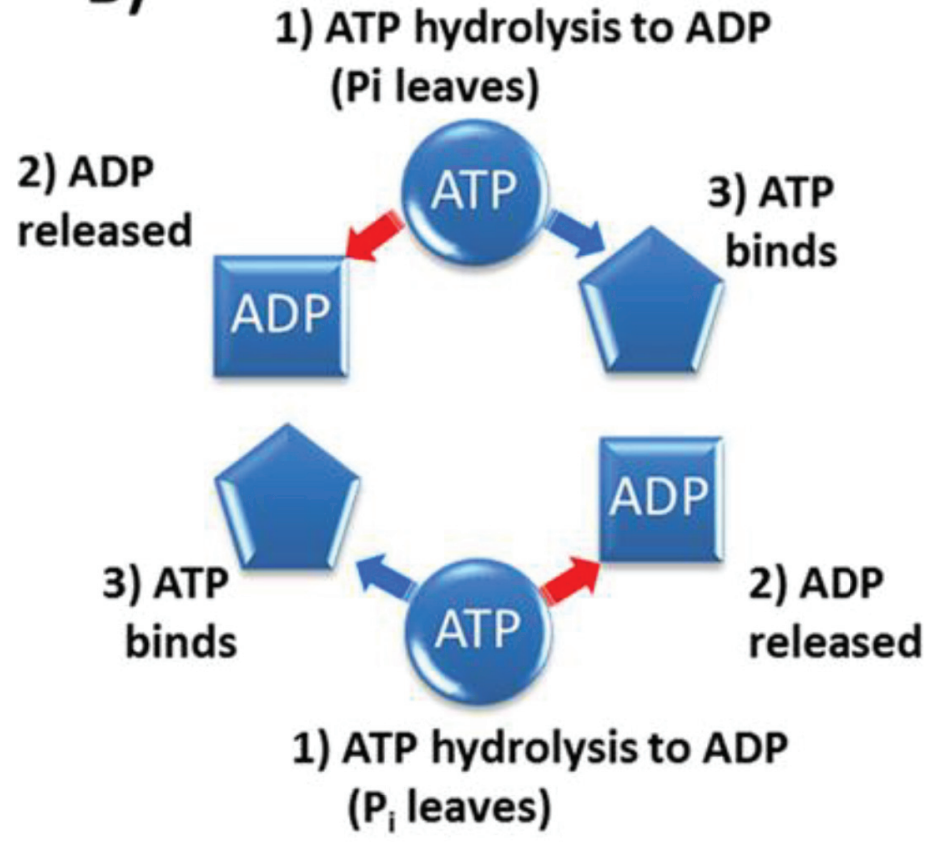

Figure 1.2: Nucleotide binding exchange model for the proteasomal ATPases. A) Three possible patterns by which a pair of ATP molecules can bind to a hexameric ring. B) Our initial model describing the binding-exchange reaction for the proteasomal ATPases based on the two cooperatively-linked para-positioned subunits binding ATP. We expected that each subunit would cycle through ATP bound, ADP bound, and nucleotide-free states. The resulting ATP hydrolysis cycle was initially expected to occur in the clockwise direction based on studies of similar ATPdependent proteases (e.g. ClpX, Martin et al. Nature 2005) 
propose in Chapter 2 that ATP hydrolysis most likely progresses in a counter-clockwise fashion (see Chapter 2 for rationale).

\section{How do substrates stimulate their own degradation: A role for the CC domains?}

Interestingly, substrates are able to stimulate ATPase activity and thus their own degradation (Bech-Otschir et al. 2009). However, it is not known how substrate binding in the distant Rpn subunits is linked to the ATPase activity in the AAA+ ATPase domains. The CC domains are known to provide a flexible tether for many of the Rpn subunits of the $19 \mathrm{~S}$ to bind (Unverdorben et al. 2014), and thus, in the $19 \mathrm{~S}$ regulatory particle, ubiquitin binding is linked to the AAA+ ATPase domain through the CC domains. At the time that this dissertation was started, we envisioned that dynamic movements in the $\mathrm{CC}$ domains could regulate proteasomal activity, and the CC domains may even act as a conduit to transmit messages between the AAA+ ATPase domains and the distant Rpn subunits. Regulation by dynamic CC movements has been observed in a handful of other proteins, including the microtubule motor protein, dynein. In dynein's CCs, a small registry shift occurs in response to the nucleotide bound-state, which allows its distant microtubule binding domain to oscillate between high and low affinity states for microtubules, resulting in "walking" across microtubules (Carter 2013). Likewise, we hypothesized that a similar registry shift may occur in PAN's CCs to modulate ATPase activity and substrate unfolding. The remainder of this review will focus on the CC domains and what is known about their functions in various protein unfoldases, including the proteasomal ATPases. 


\section{CC domains}

CCs made up of 2 or more alpha helices that wrap around one another in a "knobs into holes" fashion as first described by Francis Crick in the early 1950s, where a residue ("knob") from one helix packs into the "hole" between 4 residues of another helix (Crick 1953). Right handed, dimeric CCs (the type found in proteasomal ATPases) have a repeating 7 residue consensus sequence termed a "heptad repeat". Conventionally these residues are lettered a-g, where residues 'a' \& 'd' are hydrophobic, 'e' \& ' $g$ ' are charged, and the rest are typically polar (Fig. 1.3A). Since $\alpha$-helices are 3.6 residues per turn, distorting the alpha helix by $\sim 20^{\circ}$ results in every 3.5 residues (the hydrophobic ' $a$ ' and 'd' residues) packing on the inside of the CC interface. Dimerization specificity and some stability also come from electrostatic interactions between the charged residues in the 'e' and ' $g$ ' positions, but the hydrophobic interactions between the 'a' and ' $d$ ' residues are the main stabilizing interactions in the CCs. The rest of the mainly polar residues are exposed to solvent to allow for CC solubility (Mason \& Arndt 2004). The 'a' residue has a parallel $\mathrm{C} \alpha-\mathrm{C} \beta$ bond, while the 'd' residue has a perpindicular $\mathrm{C} \alpha-\mathrm{C} \beta$ bond (Fig. 1.3B). So, the distance between 'a' residues is slightly greater than the distance between the ' $d$ ' residues. Therefore, residues with beta branching (e.g. V, I, T) are favored in the 'a' position, while non-beta branched amino acids are favored in the 'd'

position (e.g. L, A) (Tripet et al. 2000). For the same reason, disulfide bonds in the 'd' positions result in a highly stable structure, however, disulfides in the 'a' position place strain on the CC and often cause destabilization and misfolding (Zhou et al. 1993). 


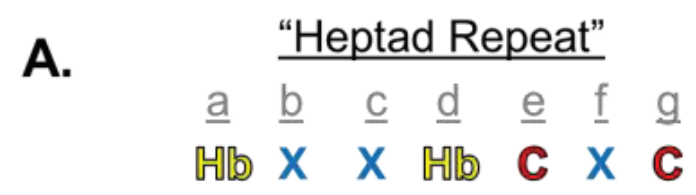

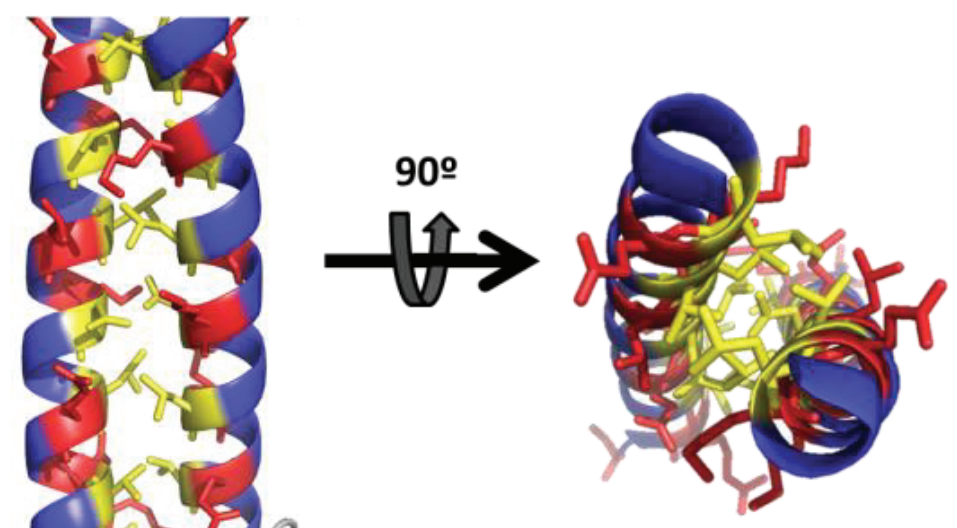

B. ' $a$ ' residues, parallel $\mathrm{C} \alpha-\mathrm{C} \beta$ bond
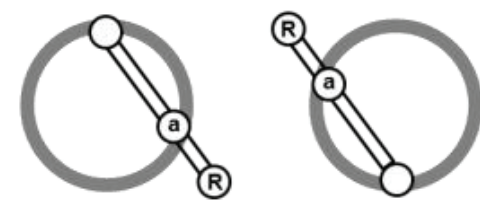

' $d$ ' residues, Perpendicular $\mathrm{C} \alpha-\mathrm{C} \beta$ bond

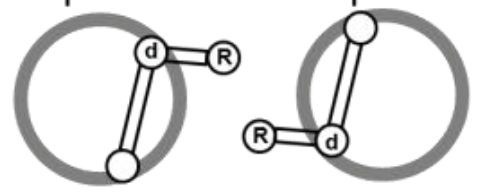

Figure 1.3. The basic structure of dimeric, right handed coiled-coil domains. A. The Consensus sequence for a CC is a heptad repeat. Hydrophobic residues (yellow) pack on the inside of the CC interface while charged residues (red) add additional stability and dimerization specificity via electrostatic interactions, and the rest of the residues (blue) are exposed to solvent and thus typically polar. $\mathbf{B}$. The ' $a$ ' residues have a parallel $C \alpha-C \beta$ bond and the ' $d$ ' residues have a perpendicular $C \alpha-$ $\mathrm{C} \beta$. 
At the time that Crick first described CCs, they were thought to comprise mainly fibrous proteins and rigid structures such as keratin (hair) and fibrin (blood clots). However, new functions have been attributed to CCs and they have been shown to be capable of dynamic movements that can regulate the functions of proteins (Parry et al. 2008). For example, a slight unwinding in kinesin's CCs allows it the flexibility to walk across microtubules, but also provides enough rigidity to retain kinesin's structure (Tripet et al. 1997). Influenza hemaglutinin's CC undergoes a pH-dependent switching between uncoiling \& recoiling, which allows it to fuse with the membrane of the endosome when exposed to its acidic environment (Lupas 1996). Finally, multiple proteins (including dynein) have been shown to undergo shifts in their dimerization registry (e.g. $1 / 2$ heptad registry shifts) that regulate various aspects within these proteins (Kon et al. 2009). One theme among dynamic CCs is that they are rigid enough to help retain structure, but flexible enough to allow for movement of protein domains and/or send signals to distant domains via their movements.

\section{The role of $\mathrm{CCs}$ in substrate binding}

Proteasomal CCs have been thought to be involved in substrate binding simply based on the location of the CCs above the central pore and the fact that CCs are known protein binding motifs. Furthermore, the $\mathrm{N}$-domains of other related AAA+ ATPases are known to be involved in substrate binding (Zhang et al. 2004). Recent cryo-EM images of the proteasome reveal that there are various subunits that are associated with the CCs, thus, it appears that the proteasomal CCs have evolved to bind these specific proteins. Furthermore, some non-ubiquitinated substrate proteins such as c-Fos and 
hepatitis BX protein are recognized by specific Rpt CCs (Wang et al. 1996; Zhang et al. 2000). It is plausible that the CCs from PAN and Mpa are able to recognize substrates in a similar way. In support of this, some species of archaea have multiple isoforms of PAN in which the only difference is the sequence of their CC domains (Reuter et al. 2004). Studies have not been conducted concerning their substrate specificity, but it is likely that these different isoforms serve to recognize different subsets of substrates. Furthermore, chimeras were generated in which PAN's CCs were replaced with various other CCs. PAN worked normally for the activities tested, but with different protein substrate preferences. Altering the $\mathrm{OB}$ domains also seems to alter $\mathrm{CC}$ specificity toward protein substrates (Djuranovic et al. 2009), which suggests that the CC conformations are directly linked to conformations of the OB domains (and perhaps this even could extend to the AAA+ ATPase domains).

In mycobacteria there is a degradation tag called "prokaryotic ubiquitin-like protein" (Pup). Pup is an unstructured protein that forms an a-helix upon binding the CCs of Mpa. In Mpa crystal structures where the AAA+ ATPase domain was removed, Pup was able to bind all 3 CCs of Mpa (i.e. the stoichiometry of Pup:Mpa is 3:1) (Wang et al. 2010). However, in vitro experiments determined that Pup:Mpa stoichiometry is only 1:1 (Sutter et al. 2009). This suggests that the ATPase domains impose some sort of allosteric restriction on the CC domains that allow no more than 1 out of 3 CCs to bind substrate at any given point, and that relieving this restriction by truncating the ATPase domain allows for all 3 of Mpa's CCs to bind substrates. 


\section{The role of CCs in chaperone activity and ATPase activity}

When this dissertation was started, chaperone activity (the prevention of aggregation of substrates) was the only function that has been definitively attributed to proteasomal CCs. PAN CC truncation mutants displayed decreased chaperone activity of substrates, and truncation down to 1 heptad completely abrogated chaperone activity. Interestingly, ATP is not required for chaperone activity, since after removal of the AAA+ ATPase domain, the CC-OB domains still exhibit chaperone activity. However, ATP binding does seem to stabilize the CCs in a conformation that is favorable for optimal chaperone activity, since addition of nonhydrolyzable ATPYS did seem to enhance chaperone activity (Benaroudj \& Goldberg 2000; Djuranovic et al. 2009). The fact that ATP might modulate CC chaperone activity is an interesting concept, and also suggests that the reciprocal may be true (i.e. the CCs may regulate ATPase function). Further evidence for CC modulation of ATPase activity is that modification at the very $\mathrm{N}$-terminus of PAN's CC results in a mutant that has $60 \%$ less activity than WT PAN. Furthermore, CC truncations to a length of 2 heptads alters ATPase activity and nucleotide specificity. CC truncations have slightly increased ATPase activity $(\sim 150 \%$ of WT). This increase in activity may be analogous to disconnecting the drivetrain from the engine of a car- the reduced resistance to the engine would allow it to rev faster with less effort. The 2 heptad CC truncation was also more promiscuous in the nucleotides it chooses to bind and hydrolyze (WT PAN can only utilize ATP \& CTP, whereas PAN partial CC truncations can utilize ATP, CTP, ITP, GTP, TTP, and UTP) (Wilson et al. 2000). 


\section{Conclusions}

In summary, the CC domains of PAN likely have many roles that have yet to be uncovered. Substrates often bind to the N-domains of AAA+ ATPase proteins, and it has been shown that substrates are able to bind the CCs of both the $19 \mathrm{~S}$ and Mpa, and thus it is likely that substrates bind to PAN's CCs. Furthermore, it is known that substrates are able to stimulate ATPase activity, and there is reasonable evidence to suggest allosteric communication between the CC and AAA+ ATPase domains. There is also evidence that the $\mathrm{CC}$ domains are able to regulate the specificity and rate of nucleotide hydrolysis in the AAA+ ATPase domains, as truncating the CCs causes deregulation of the AAA+ATPase. CC domains from other proteins have been shown to undergo dynamic movements in response to specific signals, and these dynamic movements can propogate long-range signals to other protein domains. Given what is known about the PAN's CC domains, their substrate binding capacity, as well as their ability to regulate the AAA+ ATPase domain (and thus all of PAN's activities), we hypothesized that dynamic CC movements are responsible for responding to various signals from bound substrates and "communicating" these signals to the AAA+ ATPase domains. In the following chapters, we found that although the CC domain conformations were indeed fundamental to the proper functioning of the proteasomal ATPases, conformational changes did not appear to be necessary in all of the CC domains. Rather, we find that conformational switching of the $\mathrm{CC}$ domains regulate ATP hydrolysis rates, but the CCs do not appear to be "communication conduits", at least not in the way that we envisioned (e.g. PAN's CC domains do not function as the "on/off" switch that's been observed in other coiled-coils like dynein). We find that PAN's 
CCs can switch between high and low activity states, and it seems that the CC domains mainly function to impart flexible structural roles by both stabilizing the hexameric state of the ATPase ring, and that different $\mathrm{CC}$ conformations can either enhance or inhibit the conformational changes that drive unfolding. Due to these unexpected observations, we extended the overall goal of this project to not only elucidating the conformations of the $\mathrm{CC}$ domains, but also to determine how allosteric interactions within the proteasomal ATPases catalyze protein degradation. Chapter 2 focuses on the nucleotide binding patterns of proteasomal ATPases, Chapter 3 focuses on the proteasome's processivity, and its ability to maintain grip on substrates, and finally Chapter 4 focuses on PAN's CC domains. In this dissertation, we hypothesized that we could elucidate the mechanism of substrate processing in the proteasomal ATPases by using PAN as a model system. Though chapters 2-4 may initially appear unrelated, in Chapter 5 we will show how we can utilize the CC mutants (made in Chapter 4) to experimentally desymmeterize a homohexamer with minimal structural perturbations. We will show how, with single point mutations to PAN's CC domains, we can distinguish between subunits in a homohexamer based on the conformations they adopt, thus allowing us to answer lingering questions regarding PAN's functionings that were impossible to answer in Chapters 2-3 due to its homohexameric nature.

\section{References}

Bech-Otschir, D., Helfrich, A., Enenkel, C., Consiglieri, G., Seeger, M., Holzhütter, H.G., et al. (2009). Polyubiquitin substrates allosterically activate their own degradation by the 26S proteasome. Nat. Struct. Mol. Biol. 16, 219-25. doi:10.1038/nsmb.1547.

Benaroudj, N., and Goldberg, a L. (2000). PAN, the proteasome-activating nucleotidase from archaebacteria, is a protein-unfolding molecular chaperone. Nat. Cell Biol. 2, 
833-9. doi:10.1038/35041081.

Carter, A. P. (2013). Crystal clear insights into how the dynein motor moves. J. Cell Sci. 126, 705-13. doi:10.1242/jcs.120725.

Crick, F. H. (1953). The packing of alpha helices: simple coiled-coils. Acta Crystallogr. 6, 689-697.

Djuranovic, S., Hartmann, M. D., Habeck, M., Ursinus, A., Zwickl, P., Martin, J., et al. (2009). Structure and Activity of the N-terminal Substrate Recognition Domains in Proteasomal ATPases. Mol. Cell 34, 580-590. doi:10.1016/j.molcel.2009.04.030.

Gorbea, C., Taillandier, D., and Rechsteiner, M. (2000). Mapping Subunit Contacts in the Regulatory Complex of the 26 S Proteasome. S2 AND S5b FORM A TETRAMER WITH ATPase SUBUNITS S4 and S7. J. Biol. Chem. 275, 875-882. doi:10.1074/jbc.275.2.875.

lyer, L. M., Leipe, D. D., Koonin, E. V, and Aravind, L. (2004). Evolutionary history and higher order classification of AAA+ ATPases. J. Struct. Biol. 146, 11-31. doi:10.1016/j.jsb.2003.10.010.

Jankowska, E., Stoj, J., Karpowicz, P., Osmulski, P. A., and Gaczynska, M. (2013). The proteasome in health and disease. Curr. Pharm. Des. 19, 1010-1028.

Kish-Trier, E., and Hill, C. P. (2013). Structural biology of the proteasome. Annu. Rev. Biophys. 42, 29-49. doi:10.1146/annurev-biophys-083012-130417.

Kon, T., Imamula, K., Roberts, A. J., Ohkura, R., Knight, P. J., Gibbons, I. R., et al. (2009). Helix Sliding in the Stalk Coiled Coil of Dynein Couples ATPase and Microtubule Binding. Nat. Struct. Mol. Biol. 16, 325-33. doi:10.1038/nsmb.1555.

Lupas, A. (1996). Coiled coils: New Structures and New Functions. Trends Biochem. Sci. 21, 375-82. Available at: http://www.ncbi.nlm.nih.gov/pubmed/8918191.

Martin, A., Baker, T. a, and Sauer, R. T. (2008). Diverse pore loops of the AAA+ CIpX machine mediate unassisted and adaptor-dependent recognition of ssrA-tagged substrates. Mol. Cell 29, 441-50. doi:10.1016/j.molcel.2008.02.002.

Mason, J. M., and Arndt, K. M. (2004). Coiled Coil Domains: Stability, Specificity, and Biological Implications. Chembiochem 5, 170-6. doi:10.1002/cbic.200300781.

Parry, D. a D., Fraser, R. D. B., and Squire, J. M. (2008). Fifty Years of Coiled-coils and Alpha-helical Bundles: A Close Relationship Between Sequence and Structure. J. Struct. Biol. 163, 258-69. doi:10.1016/j.jsb.2008.01.016.

Rechsteiner, M., Hoffman, L., and Dubiel, W. (1993). The multicatalytic and 26 S proteases. J. Biol. Chem. 268, 6065-8. Available at: http://www.ncbi.nlm.nih.gov/pubmed/8646844. 
Reuter, C. J., Kaczowka, S. J., and Maupin-furlow, J. A. (2004). Differential Regulation of the PanA and PanB Proteasome-Activating Nucleotidase and 20S Proteasomal Proteins of the Haloarchaeon Haloferax volcanii Differential Regulation of the PanA and PanB Proteasome-Activating Nucleotidase and 20S Proteasomal Protein. J. Bacteriol. 186, 7763-7772. doi:10.1128/JB.186.22.7763.

Sen, M., Maillard, R. A., Nyquist, K., Rodriguez-Aliaga, P., Pressé, S., Martin, A., et al. (2013). The ClpXP Protease Unfolds Substrates Using a Constant Rate of Pulling but Different Gears. Cell 155, 636-646. doi:10.1016/j.cell.2013.09.022.

Smith, D. M., Chang, S.-C., Park, S., Finley, D., Cheng, Y., and Goldberg, A. L. (2007). Docking of the Proteasomal ATPases' Carboxyl Termini in the 20S Proteasome's Alpha Ring Opens the Gate for Substrate Entry. Mol. Cell 27, 731-44. doi:10.1016/j.molcel.2007.06.033.

Smith, D. M., Fraga, H., Reis, C., Kafri, G., and Goldberg, A. L. (2011). ATP Binds to Proteasomal ATPases in Pairs with Distinct Functional Effects, Implying an Ordered Reaction Cycle. Cell 144, 526-38. doi:10.1016/j.cell.2011.02.005.

Sutter, M., Striebel, F., Damberger, F. F., Allain, F. H.-T., and Weber-Ban, E. (2009). A distinct structural region of the prokaryotic ubiquitin-like protein (Pup) is recognized by the N-terminal domain of the proteasomal ATPase Mpa. FEBS Lett. 583, 31513157. doi:10.1016/j.febslet.2009.09.020.

Tripet, B., Vale, R. D., and Hodges, R. S. (1997). Demonstration of Coiled-Coil Interactions within the Kinesin Neck Region Using Synthetic Peptides. IMPLICATIONS FOR MOTOR ACTIVITY. J. Biol. Chem. 272, 8946-8956. doi:10.1074/jbc.272.14.8946.

Tripet, B., Wagschal, K., Lavigne, P., Mant, C. T., and Hodges, R. S. (2000). Effects of side-chain characteristics on stability and oligomerization state of a de novodesigned model coiled-coil: 20 amino acid substitutions in position "d". J. Mol. Biol. 300, 377-402. doi:10.1006/jmbi.2000.3866.

Unverdorben, P., Beck, F., Led, P., Schweitzer, A., Pfeifer, G., Plitzko, J. M., et al. (2014). Deep Classification of a Large Cryo-EM Dataset Defines the Conformational Landscape of the 26S Proteasome. Proc. Natl. Acad. Sci. 111, 5544-5549. doi:10.1073/pnas.1403409111.

Wang, T., Darwin, K. H., and Li, H. (2010). Binding-induced folding of prokaryotic ubiquitin-like protein on the Mycobacterium proteasomal ATPase targets substrates for degradation. Nat. Struct. Mol. Biol. 17, 1352-7. doi:10.1038/nsmb.1918.

Wang, W., Chevray, P. M., and Nathans, D. (1996). Mammalian Sug1 and c-Fos in the nuclear 26 S proteasome. Proc. Natl. Acad. Sci. U. S. A. 93, 8236-40. Available at: http://www.pubmedcentral.nih.gov/articlerender.fcgi?artid=38653\&tool=pmcentrez\& rendertype $=$ abstract. 
Wilson, H. L., Ou, M. S., Aldrich, H. C., and Maupin-Furlow, J. (2000). Biochemical and Physical Properties of the Methanococcus jannaschii 20S Proteasome and PAN, a Homolog of the ATPase (Rpt) Subunits of the Eucaryal 26S Proteasome. J. Bacteriol. 182, 1680-1692. Available at:

http://www.pubmedcentral.nih.gov/articlerender.fcgi?artid=94466\&tool=pmcentrez\& rendertype=abstract.

Yu, Y., Smith, D. M., Kim, H. M., Rodriguez, V., Goldberg, A. L., and Cheng, Y. (2010). Interactions of PAN's C-termini with archaeal 20S proteasome and implications for the eukaryotic proteasome-ATPase interactions. EMBO J. 29, 692-702. doi:10.1038/emboj.2009.382.

Zhang, F., Hu, M., Tian, G., Zhang, P., Finley, D., Jeffrey, P. D., et al. (2009). Structural Insights into the Regulatory Particle of the Proteasome from Methanocaldococcus jannaschii. Mol. Cell 34, 473-484. doi:10.1016/j.molcel.2009.04.021.

Zhang, X., Stoffels, K., Wurzbacher, S., Schoofs, G., Pfeifer, G., Banerjee, T., et al. (2004). The N-terminal coiled coil of the Rhodococcus erythropolis ARC AAA ATPase is neither necessary for oligomerization nor nucleotide hydrolysis. J. Struct. Biol. 146, 155-65. doi:10.1016/j.jsb.2003.10.020.

Zhang, Z., Torii, N., Furusaka, a, Malayaman, N., Hu, Z., and Liang, T. J. (2000). Structural and functional characterization of interaction between hepatitis $B$ virus $X$ protein and the proteasome complex. J. Biol. Chem. 275, 15157-65. doi:10.1074/jbc.M910378199.

Zhou, N. E., Kay, C. M., and Hodges, R. S. (1993). Disulfide Bond Contribution to Protein Stability: Positional Effects of Substitution in the Hydrophobic Core of the Two-stranded Alpha-helical Coiled-coil. Biochemistry 32, 3178-3187.

Zwickl, P., Ng, D., Woo, K. M., Klenk, H. P., and Goldberg, a L. (1999). An archaebacterial ATPase, homologous to ATPases in the eukaryotic $26 \mathrm{~S}$ proteasome, activates protein breakdown by $20 \mathrm{~S}$ proteasomes. J. Biol. Chem. 274, 26008-26014. doi:10.1074/jbc.274.37.26008. 
Chapter 2: ATP Binding to neighboring subunits and intersubunit coupling underlie proteasomal ATPase function 


\section{Preface}

This chapter is a continuation of the research conducted on proteasomal ATPases which revealed that PAN, a homohexamer, binds to only two ATPs and 2 ADPs at a time (Smith et al. 2011). Up until this point, two fundamental questions regarding the function of these ATPase complexes persisted: 1) What is their spatial and temporal mode of ATP-binding and hydrolysis around their multimeric ring structure, and 2) How is the chemical energy obtained from ATP coupled to mechanical work on their substrates? We believed that understanding the answer to these two questions was necessary to build a model that explained their mechanism of operation. At the time, the answer to both of these questions had not been addressed for the ATPases of the proteasome nor had these mechanisms been established in depth for any molecular motor.

In this chapter, we used FRET in a novel way to measure the distance between bound ATP in these proteasomal ATPases in solution, which had not been done before for any AAA ATPase. We surprisingly found that ATP binds exclusively to neighboring subunits. These results demonstrated that ATP binding is specific and ordered, and supported a sequential rather than probabilistic model for ATP-hydrolysis. We further proposed that ATP binding to one subunit triggers substrate and $20 \mathrm{~S}$ binding in its neighboring subunit, showing that ATP-binding effects occur "in trans". Based on our model, this intersubunit allostery is essential for substrate translocation, and is explained by a mechanochemical hand-over-hand model, which we hypothesize proceeds in a counter-clockwise fashion. 
Last, to distinguish between probabilistic and sequential models we assess the dynamics of ATP and ADP off rates using pre-steady state techniques. We showed that ATP-binding and leaving regularly occurs between ATP hydrolysis events, explaining how a purely sequential ATPase can skip subunits when mutations are introduced. This explains how some single subunit mutations can have minimal impact on the function of the proteasomal ATPases and aids interpretation of the recently proposed probabilistic model for another related ATP-dependent protease (ClpX) by suggesting a thermodynamic equilibrium explanation for this behavior.

The work presented in this chapter resulted in a $2^{\text {nd }}$ author publication for me in Nature Communications, which was co-written by Dr. Smith and I. In addition to writing this manuscript, I performed ATPase assays, ATP-PAN-binding experiments (including those quantifying stoichiometry and cooperativity, and the PAN-20S experiments). In order to make more clear my contributions to this project, I have initialed "AS" in red to indicate which figure panels I prepared in this manuscript.

Significantly, my contributions led to us revising our original arginine finger model. Initially, we proposed that only one arginine finger was necessary for ATP hydrolysis, however, after spending a great deal of time working to answer reviewer's concerns after our $1^{\text {st }}$ submission, I discovered that our PAN preparations contained a highly active ATPase contaminant that was not visible on SDS-PAGE, but whose activity could be observed following separation via size exclusion (See Fig. S2.6). This required me to repeat most experiments in this paper with newly purified PAN (contaminant-free) to determine the validity of previous results. Though I repeated most experiments that appear in the manuscript, I only credit my contributions in experiments 
whose results changed after using the more highly purified PAN. Ultimately, my contribution led to us revising our theory on the role of PAN's Arginine fingers. Based on these new insights we were able to conclude that both arginines are required for ATPase activity (not just one).

Kim, YC, Snoberger A, Schupp, J, and Smith DM. (2015) "ATP binding to neighbouring subunits and intersubunit allosteric coupling underlie proteasomal ATPase function", Nature Communications 6, 8520. doi:10.1038/ncomms9520.

This chapter has been reprinted here under Creative Commons Attribution 4.0 International License. 


\section{Abstract}

The primary functions of the proteasome are driven by a highly allosteric ATPase complex. ATP-binding to only two subunits in this hexameric complex triggers: substrate binding, ATPase-20S association, and 20S gate-opening. However, we don't know how ATP-binding and hydrolysis spatially and temporally coordinates these allosteric effects to drive substrate translocation into the 20S. Here, we use FRET to show that the proteasomal ATPases from eukaryotes (RPTs) and archaea (PAN) bind to ATP with high affinity at neighboring subunits, which complements the well-established spiral-staircase topology of the 26S ATPases. We further show that two conserved arginine fingers in PAN located at the subunit interface work together as a single allosteric unit to mediate the allosteric effects of ATP-binding without altering the nucleotide binding pattern. Rapid kinetics analysis also shows that ring resetting of a sequential hydrolysis mechanism can be explained by thermodynamic equilibrium binding of ATP. These data support a model whereby these two functionally distinct allosteric networks cooperate to translocate polypeptides into the $20 \mathrm{~S}$ for degradation. 


\section{Introduction}

The $26 \mathrm{~S}$ proteasome is an ATP-dependent multisubunit protease complex that degrades polyubiqutinated proteins in a regulated manner. This $2.5 \mathrm{MDa}$ compartmentalized protease is composed of $\sim 33$ distinctive subunits in two major subcomplexes, the $20 \mathrm{~S}$ core particle (CP) and the 19S regulatory particle (RP/PA700). The central regulatory hub of the $26 \mathrm{~S}$ is its hexameric ATPases complex (Rpt1-6), which is located at the base of the 19S. The N-terminal side (coiled coil domain) of the ATPase ring is intimately integrated with other subunits that are involved with scaffolding, ubiquitin chain binding and processing. The C-terminal side of the ATPase associates with the $20 \mathrm{~S}$ proteasome via its $\mathrm{C}$-terminal $\mathrm{HbYX}$ motif, which induces gate opening in the $20 \mathrm{~S}$ to promote substrate entry. This architecture places the ATPase ring in a position where it can accept protein substrates on its $\mathrm{N}$-terminal side and then, in an ATPdependent manner, translocate them through its central pore and into the $20 \mathrm{~S}$ for their degradation ${ }^{1-4}$. Archaea also have a proteasomal ATPase complex that is homologous to Rpt16 called PAN, which binds to and similarly regulates the archaeal 20 S proteasome.

Recent cryo-EM analyses of the $26 \mathrm{~S}$ have revealed that large conformational changes occur in the 195 when the ATPases bind ATPYS or substrates ${ }^{5-7}$. This similar ATP-bound and substrate-bound state is thought to be a degradation competent conformation. The binding of ATP (but not ADP) to RPT1-6 or PAN triggers several essential steps that are required for protein degradation: 1) substrate binding ${ }^{8,9}$, 2) 19S$20 \mathrm{~S}$ or PAN-20S association ${ }^{10,11}$, and 3) opening of the $20 \mathrm{~S}$ substrate gate ${ }^{10,11}$. Therefore, ATP-binding allosterically regulates conformational changes on both the Nand C-terminal sides of the ATPase ring. The proteasomal ATPases unfold substrates when an unstructured region of the substrate binds to the ATPases' pore loops, which 
pull on the substrate upon ATP hydrolysis ${ }^{12-14}$. Substrate binding to these pore loops requires ATP to be bound, since empty or ADP-bound complexes do not bind to substrates $^{8,9,15}$. Conformational changes in the pore loops (due to ATP hydrolysis) translocate the peptide though the ATPase ring into the 20S, causing substrate unfolding $^{12,16}$. The challenge is to understand how this complex network of subunitsubunit interactions allows ATP-binding and hydrolysis to drive and coordinate the allosteric conformational changes that catalyze this process leading to substrate degradation.

ATP-binding controls the C-terminal side of the ATPase ring by causing the HbYX motif to bind pockets between the 20S a-subunits, allowing 19S-20S and PAN$20 \mathrm{~S}$ complex formation and $20 \mathrm{~S}$ gate opening ${ }^{17-20}$. The $\mathrm{HbYX}$ motif cannot associate with the $20 \mathrm{~S} \alpha$-subunits unless ATP binds to the ATPase. Though this mechanism is not completely understood, it's thought that ATP-binding causes allosteric changes that allow association with the $\alpha$-ring ${ }^{17,18}$. The averaged structures from cryo-EM analyses of the 26S (with ATP or ATPYS) showed densities for all three HbYX motifs bound to the $20 S$, confirming their necessity for 20 S-binding ${ }^{5,21,22}$. However, the specific mechanistic details of this ATP-dependent $26 \mathrm{~S}$ assembly processes and gating functions are not clearly understood ${ }^{10,11,15,17,19,20,23,24}$. Understanding how ATP-binding allosterically triggers ATPase-20S association via the HbYX motif will be key to understanding the dynamics of how these ATPase rings bind to the $20 \mathrm{~S}$ to inject substrates.

Because ATP-binding and hydrolysis to ADP are both essential for proteasome function, understanding how these events are coordinated both spatially and temporally 
is critical to understand how work is done on substrates. Even though PAN has six identical subunits it's been shown to have two high affinity ATP-binding sites, two low affinity sites, and two sites that cannot bind ATP when the high and low affinity sites are occupied $^{8}$. ATP-binding to the two high affinity sites produced maximal function (e.g., $20 \mathrm{~S}$ gate-opening or substrate binding), while ATP-binding to the low affinity sites reduced function. Based on similar functional studies the 26S ATPases appear to bind to ATP with nearly identical allosteries as $\mathrm{PAN}^{8}$. These and other data indicate that the proteasomal ATPases' highest functional state had two bound ATPs, two bound ADPs, and two empty sites. Therefore, an extensive allosteric system linking the ATPase subunits must exist that controls how many ATPs bind around the ATPase ring. A working model for the ATP-binding/exchange reaction was built based on these data using symmetry considerations mirroring the nucleotide binding pattern in the crystal structure of the T7 gene helicase ${ }^{25}$. This working model suggested that ATP binds to para position subunits $\left(180^{\circ}\right.$ from one another) in the ring, but the results also did not exclude the possibility of other paired patterns of ATP-binding ${ }^{8}$. However, the strictly imposed allosteries indicated that a patterned mode of ATP-binding and hydrolysis is likely, which fits well with a sequential mechanism for ATP hydrolysis ${ }^{26}$.

A related AAA+ ATPase, ClpX, has similar types of high, low, and no affinity ATP-binding subunits as the proteasomal ATPases, but any specific organizational pattern of ATP hydrolysis has not been established, though various models have been presented ${ }^{27,28}$. Interestingly, ClpX is capable of hydrolyzing ATP (at impaired rates) with only a single fully functional (WT) subunit. These results were interpreted to indicate 
that ClpX subunits have a degree of autonomy, which may allow ClpX to function in a probabilistic manner ${ }^{29}$. Because the pattern of ATP-binding and hydrolysis is what coordinates and controls the movements of the ATPase subunits, the determination of how these binding sites are organized spatially is essential to understanding how the proteasomal ATPases function to translocate substrates. Determining if a specific pattern of ATP-binding exists and what it might be is the first step to understanding how these molecular machines work.

AAA+ ATPases in all domains of life use six conserved motifs for function; i.e., Walker A, Walker B, Sensor 1, Arginine finger, Sensor 2, and Pore loops ${ }^{30-32}$. Out of these six motifs, only the arginine finger is in a position to allow for allosteric communication between subunits ${ }^{33,34}$. The arginine finger in the proteasomal ATPases projects from one subunit into its neighboring subunit's nucleotide binding site, and thus it functions in "trans". In contrast, some AAA+ members, such as ClpX, do not appear to contain a functional trans arginine finger, but rather have a Sensor 2 arginine that functions in "cis" 35,36 . The proteasomal ATPases have two conserved "trans" arginines and either may function as an arginine finger. Since a single arginine is sufficient to catalyze ATP hydrolysis in some members, it's not understood why some ATPases contain double arginines in this position.

Despite recent advances in determining the structure of the $26 \mathrm{~S}$ proteasome and its dynamics, a detailed mechanistic understanding of how ATP-binding and hydrolysis coordinate the conformational changes that generate a functional machine is not understood. In this study we are interested in how ATP-binding and hydrolysis 
allosterically regulate the function of the proteasomal ATPases. We found that PAN and the mammalian 26S ATPases bind to ATP with an ordered neighbor-binding pattern (Ortho) and that the conserved arginine finger residues in PAN play a central allosteric role in controlling the fundamental mechanisms that catalyze proteasome function. In addition, these findings support an allosteric model, describing how ATP-binding and hydrolysis are coordinated by separate allosteric systems that control the conformational changes that drive unfolding and translocation of substrates into the proteasome for degradation. 


\section{Results}

ATP binds to neighboring subunits ("Ortho" pattern) in the proteasomal ATPases The proteasomal ATPases have two high affinity ATP-binding sites ${ }^{8}$. To determine the position of these high affinity subunits within the hexameric complex, we monitored Förster Resonance Energy Transfer (FRET) between fluorescent nucleotides bound to the high affinity sites using mant-ATP (m-ATP; Ex: 360, Em: 450) as the donor and TNP-ATP (t-ATP; Ex: 470, Em: 570; Förster critical distance $\left(R_{0}\right): 40 \AA^{37}$ as the acceptor nucleotide (Fig. S2.1). To measure the various distances between nucleotide binding sites in the crystal structure, we measured the distances between the alanine residue in Sensor 2 motif (GAE/D) (PDB;4CR4). This residue was chosen because it binds adjacent to the ribose ring of the nucleotide, which contains the Mant or TNP moiety ${ }^{38}$. Fig. S2.2 shows the estimated average distance between all pairs of ATP-binding sites in the $26 \mathrm{~S}$ proteasomal ATPases.

To prevent the hydrolysis of ATP during the FRET experiment, we generated a Walker B mutant (E271Q) of PAN. ATP was shown to bind to PAN-E271Q in a biphasic fashion exhibiting both high and low affinity binding sites, as was previously observed using WT PAN and the non-hydrolyzable ATP analog ATPYS ${ }^{8}$ (Fig. S2.3A). Because FRET efficiency will be determined in a mixed state ( $m-A T P+t-A T P)$, it is important to know if their affinities differ to be able to calculate differential occupancy. ATP, m-ATP and t-ATP each bound to PAN-E271Q with remarkably similar affinities (Fig. S2.3A-C), indicating that fluorescent labeling of the nucleotide does not affect binding to PAN. 
To determine FRET efficiency in the two-bound state, we monitored m-ATP (donor) fluorescence decrease in the presence of t-ATP (acceptor). To minimize the amount of unbound nucleotide while also maximizing the population of PAN in the 2bound state, we used $2 \mu \mathrm{M}$ of total nucleotide with $1 \mu \mathrm{M}$ PAN hexamer $\left(\mathrm{PAN}^{6}\right)$. This concentration of nucleotide is saturating at $\sim 20 \mathrm{X}$ the $\mathrm{K}_{\mathrm{d}}$ for the high affinity sites, and the low affinity sites will not be bound by any appreciable amount $\left(K_{d}=\sim 200 \mu M\right)$. PANE271Q was incubated with m-ATP and unlabeled ATP (no FRET condition), or with mATP and t-ATP (FRET condition). In the FRET condition m-ATP fluorescence decreases by $\sim 35 \%$, which is due to resonance energy transfer due to the TNP presence on the t-ATP (Fig. 2.1A). To ensure that the observed FRET was due to nucleotide occupancy of PAN-E271Q, m-ATP and t-ATP were mixed in the same conditions without PAN, and little FRET was observed ( 1 1\%). The FRET efficiency (see experimental procedures) was $0.67+/-0.07$ (Table 1). Using the typical dipole orientation factor $\left(\kappa^{2}=2 / 3\right.$-randomly oriented) to calculate donor-acceptor distance, the observed distance between high affinity binding sites is $37+/-2 \AA$. This is likely a good estimate since the fluorophores do not alter the binding affinity, and thus do not likely bind to PAN. This measurement indicates that the high affinity binding sites are "ortho" (neighboring) subunits. To eliminate assumptions about emission dipole orientation, we further measured anisotropy values of bound m-ATP and bound t-ATP and calculated a

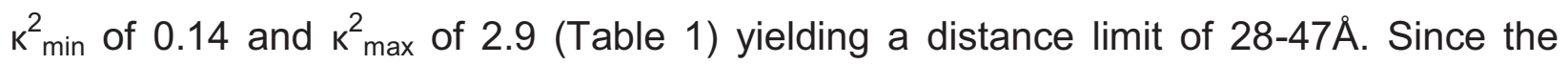
maximum possible empirical distance for this FRET pair is still $18 \AA$ shorter than the Meta position distance, this data further supports the conclusion that the high affinity sites are neighboring subunits (Fig. 2.1E-F). 

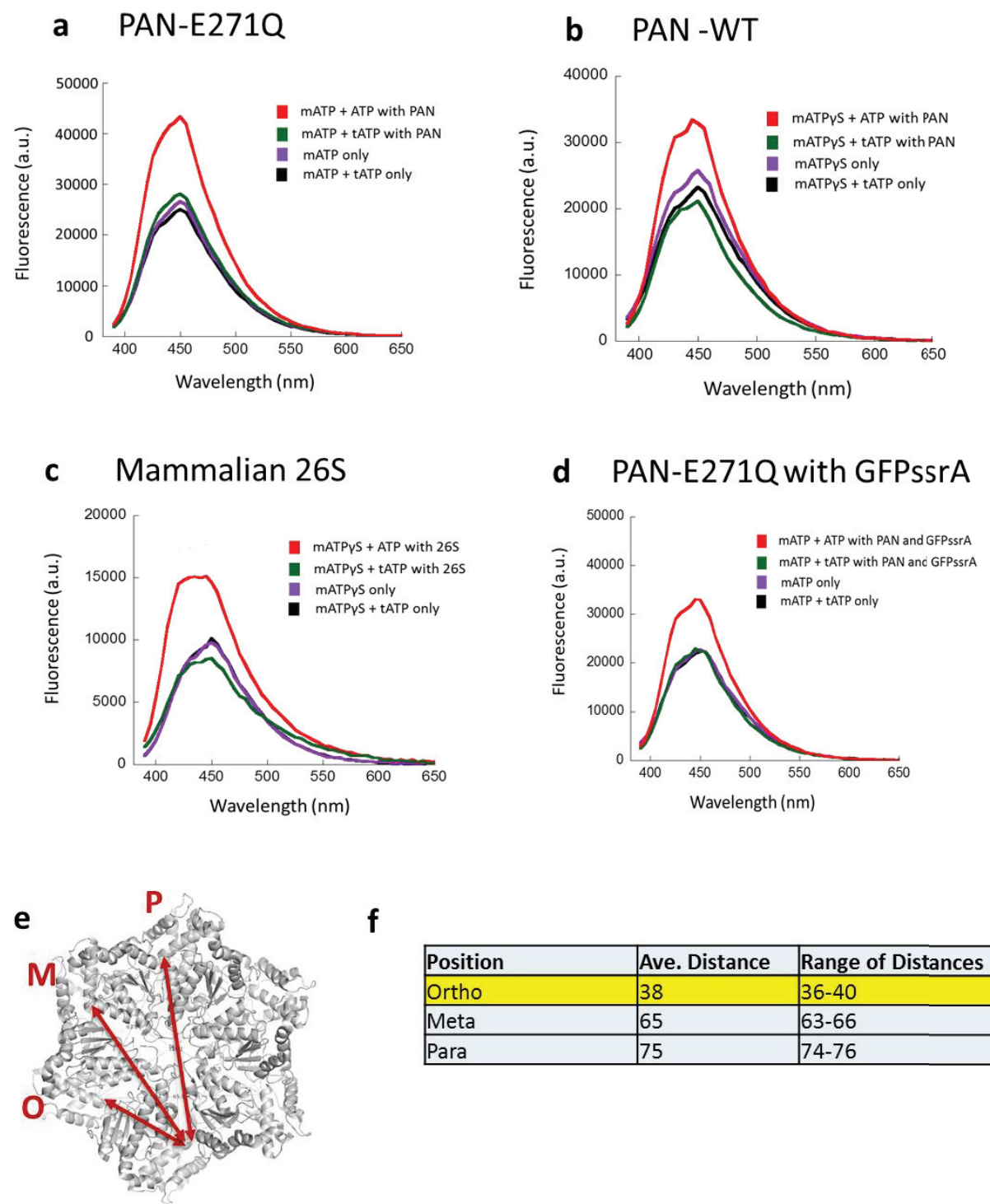

f
\begin{tabular}{|l|l|l|}
\hline Position & Ave. Distance & Range of Distances \\
\hline Ortho & 38 & $36-40$ \\
\hline Meta & 65 & $63-66$ \\
\hline Para & 75 & $74-76$ \\
\hline
\end{tabular}

Figure 2.1. ATP binds to neighboring subunits ("Ortho" pattern) in the proteasomal ATPases. A) Emission spectra of m-ATP $(1 \mu \mathrm{M})$ in the presence or absence of the indicated nucleotides $(1 \mu \mathrm{M}$ each) and PAN-E271Q $(1 \mu \mathrm{M})$ at equilibrium $\left(37^{\circ} \mathrm{C}\right)$. The FRET (with t-ATP) and no FRET conditions are shown and are color labeled. B) Same conditions as in A but with $\mathrm{m}-\mathrm{ATP} \gamma \mathrm{S}$ and WT PAN at $4^{\circ} \mathrm{C}$. C) Same conditions as in B but with bovine $26 \mathrm{~S}$ proteasome $(1 \mu \mathrm{M})$ instead of PAN. D) Same conditions as in A, but with the addition of GFP-ssrA $(1 \mu \mathrm{M})$, which was photo-bleached by UV treatment prior to assay to minimize the inner filter effect of GFP. E) Structure of the 26S proteasomal ATPases (4CR4-atomic model derived from an $8 \AA$ cryo-EM map), indicating the distance measurements between the Sensor 2 residues in the various nucleotide binding sites (O-Ortho, M-Meta, and PPara). F) Estimated average distance and ranges between Ortho, Meta and Para positioned nucleotide binding sites in the eukaryotic 26S ATPases (4CR4) corresponding to $(E)$. 
Because these FRET measurements were made in a PAN Walker B mutant we sought to verify "ortho" binding in wild-type PAN (WT PAN). To prevent ATP hydrolysis while using WT PAN, we used m-ATPYS with t-ATP at $4^{0} \mathrm{C}$. This combination minimized t-ATP hydrolysis and allowed for stable fluorescence intensity measurements within a $\sim 60 \mathrm{sec}$ time frame. Identical experiments were performed under these new conditions using WT PAN, and the distance determined by FRET was nearly identical to PANE271Q (Fig. 2.1B and Table 2.1). We also performed the same experiment with $26 \mathrm{~S}$ proteasome purified from bovine liver (Fig. 2.1C) and obtained nearly identical distance measurements as with PAN (Table 2.1) indicating that the 26S proteasomal ATPases also have "ortho" positioned high affinity binding sties. This is the first evidence to show that the high affinity ATP-binding sites in the archaeal and eukaryotic proteasomal ATPases are spatially organized in the same way. These data strongly suggest that PAN and the 26S ATPases will bind and hydrolyze ATP in a similar fashion. This "ortho" binding pattern is complementary to the helical topology of the 26S ATPases observed in several recent cryo-EM studies (see Discussion) and suggests that PAN also shares this helical topology.

It is known that substrate (e.g., GFP-ssrA) binding to PAN, which is ATP-binding dependent, stimulates its ATPase activity and that ubiquitin conjugates can act similarly on the 26S. Thus, to determine if the "ortho" binding pattern is altered in the substratebound state, we performed this same FRET experiment on PAN-E271Q with saturating amounts of photobleached GFP-ssrA bound to PAN. No change in donor-acceptor distance was observed compared to the same conditions without substrate (Fig. 2.1D 


\section{Table 2.1: FRET distance calculations between nucleotides}

\begin{tabular}{|c|c|c|c|}
\hline Experimental condition & $\begin{array}{c}\text { FRET } \\
\text { efficiency, } E\end{array}$ & $\begin{array}{l}\text { D-A distance } \\
(\AA) r\left(K^{2}=2 / 3\right)\end{array}$ & $\begin{array}{c}\text { D-A distance } \\
(\AA) r_{\min }-r_{\max }\left(K^{2} \text { limits }\right)\end{array}$ \\
\hline $\begin{array}{l}\text { PAN-E271Q }(1 \mu \mathrm{M}) \text { with } \mathrm{m} \text { - } \\
\text { ATP and t-ATP }\end{array}$ & $0.67 \pm 0.07$ & $37 \pm 2.0$ & $28-47$ \\
\hline $\begin{array}{l}\text { PAN WT }(1 \mu \mathrm{M}) \text { with } \\
\text { mATPyS and t-ATP }\end{array}$ & $0.75 \pm 0.04$ & $34 \pm 1.0$ & $27-44$ \\
\hline $\begin{array}{l}26 \mathrm{~S} \text { proteasome-WT }(1 \mu \mathrm{M}) \\
\text { with mATPyS and t-ATP }\end{array}$ & $0.77 \pm 0.08$ & $31 \pm 2.6$ & $26-44$ \\
\hline $\begin{array}{l}\text { PAN-E271Q }(1 \mu \mathrm{M})+\text { GFP- } \\
\text { ssrA }(1 \mu \mathrm{M})\end{array}$ & $0.71 \pm 0.09$ & $36 \pm 2.8$ & $28-46$ \\
\hline $\begin{array}{l}\text { PAN-R328/331A }(1 \mu \mathrm{M}) \text { with } \\
\text { m-ATP and t-ATP }\end{array}$ & $0.65 \pm 0.03$ & $37 \pm 0.8$ & $29-48$ \\
\hline
\end{tabular}

FRET, Förster resonance energy transfer; m-ATP, mant-adenosine triphosphate (donor); t-ATP, TNP-adenosine triphosphate acceptor; WT, wild type.

${ }^{*} \mathcal{}^{2}$ limits were determined by determining the steady-state and fundamental anisotropies of the bound donor (D) and acceptor (A). For example, for PANE271Q: $\mathcal{\varkappa}^{2}{ }_{\min }=0.14 ; \mathcal{\varkappa}^{2}{ }_{\max }=2.9$. 
and Table 2.1). Binding of fluorescent GFP-ssrA to PAN-E271Q was confirmed by anisotropy. This result indicates that the "ortho" ATP-binding pattern is not altered when GFP-ssrA binds to PAN, and thus is not likely to be altered during substrate translocation.

The FRET distance between high affinity sites shown here are ensemble averages. However, because the obtained distances are consistent with the minimum distances possible between nucleotide binding sites (Ortho), we can conclude that this binding pattern is the dominant binding pattern that exists in these experiments, as mixed populations with binding to the meta and/or para sites would have produced FRET distance averages consistent with either meta or para positions. We next sought to determine if the arginine finger residues of PAN are required to generate this "ortho" ATP-binding pattern since these residues have been shown to play intersubunit allosteric roles in other AAA+ ATPases ${ }^{30,33}$.

\section{Selection of the conserved arginine residues and generation of mutants}

A sequence alignment of PAN and Rpt1-6 from human and yeast proteasomal ATPases shows two highly conserved arginines at residues 328 and 331 (Fig. S2.4A). While PAN's crystal structure has been solved the subunit neighboring contacts are not shown, since it did not crystalize as a hexamer ${ }^{38}$. However, PAN's structure has been fit to cryo-EM structures of the $26 \mathrm{~S}$ proteasome and analysis of the $\mathrm{Rpt}_{2-1}$ interface (PDB: $4 B G R)^{5}$ clearly shows that these two conserved arginines in Rpt2 project towards the nucleotide binding site of Rpt1 as expected for an Arginine finger (Fig. S2.4B). Other Rpt interfaces show similar positions in the different subunit interfaces, except for the 
$\mathrm{Rpt}_{1-5}$ interface ${ }^{5}$. To systematically analyze the functional role of these two conserved arginine residues in PAN, we have generated three mutants: 1) R328A, 2) R331A and 3) the double mutant: R328/331A, (Fig. S2.4C).

\section{Both conserved arginine residues are required for ATP hydrolysis but not for ATP-binding}

To determine if these trans positioned arginines are required to generate the Ortho ATP-binding pattern, we first determined if their mutation affected PAN's ability to bind and hydrolyze ATP. The classical role of the arginine finger is to stabilize the transition state's negative charge on the gamma phosphate of ATP to catalyze its hydrolysis ${ }^{33}$. To determine if R328 and R331 fulfilled this role in PAN, we used a real-time ATPase assay using an ATP regenerating system. WT PAN hydrolyzed ATP at $\sim 1.1 / \mathrm{sec}$, which

is consistent with prior observations using end-point assays ${ }^{39}$. However, no ATPase activity could be found for any of the three different arginine mutants (Fig. 2.2A). To study if ATP could bind to these mutants we used the ATP analog, mant-ATPYS (mATPYS, which PAN does not hydrolyze). Mant-nucleotide fluorescence increases upon binding to $\mathrm{PAN}^{8}$ and it activates the same functions that ATP-binding does (i.e., PAN$20 S$ association, 20 S gate opening and substrate binding). The fluorescence intensity of m-ATPYS increased by the same amount for WT PAN and all three of the arginine mutants ( $\sim 3$ fold), under enzyme saturating conditions. Thus, while these conserved arginines are required for ATP hydrolysis they are not necessary for ATP-binding.

Prior structural studies of the 26S ATPases indicated that one of the six ATPase subunits had an arginine finger that was not positioned in the active site, while others 


\section{AS a}

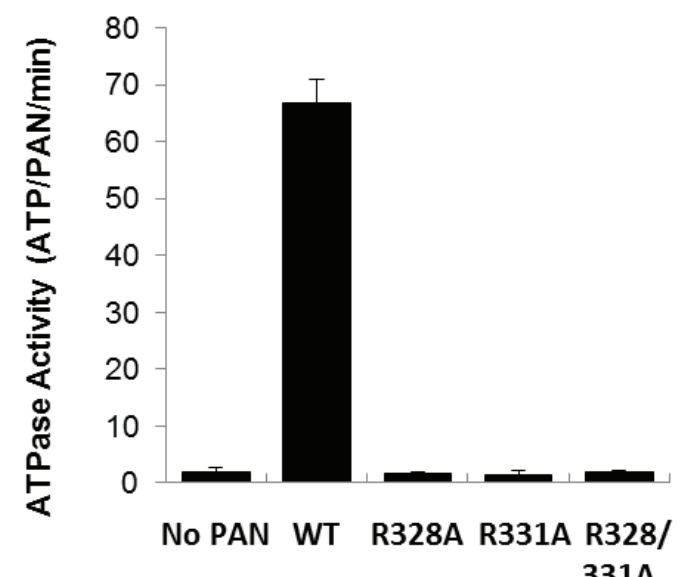

$A S^{(Y C)} \mathbf{b}$

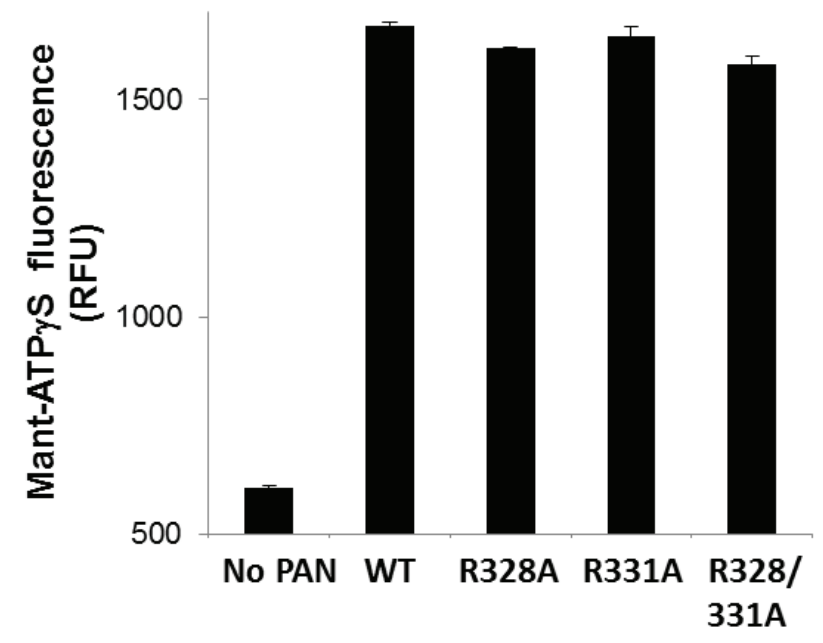

Figure 2.2. Both of the conserved arginines in PAN are required for ATP hydrolysis but not for ATP binding. A) Specific ATPase activity of WT and arginine mutants of PAN in the presence of $1 \mathrm{mM}$ ATP. ATPase rates were determined with a real-time assay. B) $\mathrm{m}-\mathrm{ATP} \gamma \mathrm{S}(15 \mathrm{nM})$ binds to PAN and the arginine mutants $(1 \mathrm{mM})$. Nucleotide binding is evident by a change in the intensity of mant fluorescence upon binding to PAN. Representative data are presented from three independent experiments +l- SD. 
were $^{5}$. Because of the important role that these arginines may play in proteasomal ATPase structure and function, we next sought to determine if binding affinities were altered. A mant-nucleotide saturation curve was generated by varying the concentration of PAN. m-ATPYS bound to WT PAN as well as all three of the arginine mutants with similar affinities (Fig. 2.3). The calculated affinities were consistent with our prior ligand binding study that quantified ${ }^{35}$ S-ATPYS binding to WT PAN ${ }^{8}$. These affinities are also very similar to the affinity of $m-A T P$ and t-ATP affinities for the PAN-E271Q (Fig. S2.3BC), indicating that the $\gamma S$ modification does not affect affinity. However, because PAN is saturating in these experiments, it's expected that only one nucleotide is bound to most of the PAN hexamers and thus cooperativity of binding cannot be reliably evaluated using this method.

\section{Neither stoichiometry nor binding pattern mutation of the conserved arginines}

PAN's optimal functional state contains 2-bound ATPs ${ }^{8}$. At low concentrations of ATP $(10 \mu \mathrm{M})$, PAN binds two molecules of ATP, but at higher concentrations $(>60 \mu \mathrm{M})$, PAN will bind to 4 ATPs (4-bound state), as long as hydrolysis is prevented by Walker B mutation (shown in Fig. S2.3A) or by using a non-hydrolyzable analog. However, this 4bound state is strained and does not function optimally ${ }^{8}$. This indicates that negative allosteries, which reduce affinity or completely prevent ATP-binding, regulates the stoichiometry and binding pattern of ATP around the ring. To determine if the nucleotide binding stoichiometry might be perturbed, we used rapid spin columns to quantify the amount of bound m-ATPYS per PAN hexamer for each variant. Two concentrations of nucleotide were used: 1) $10 \mu \mathrm{M}$, which produces the 2-bound state in WT PAN, and 2) 

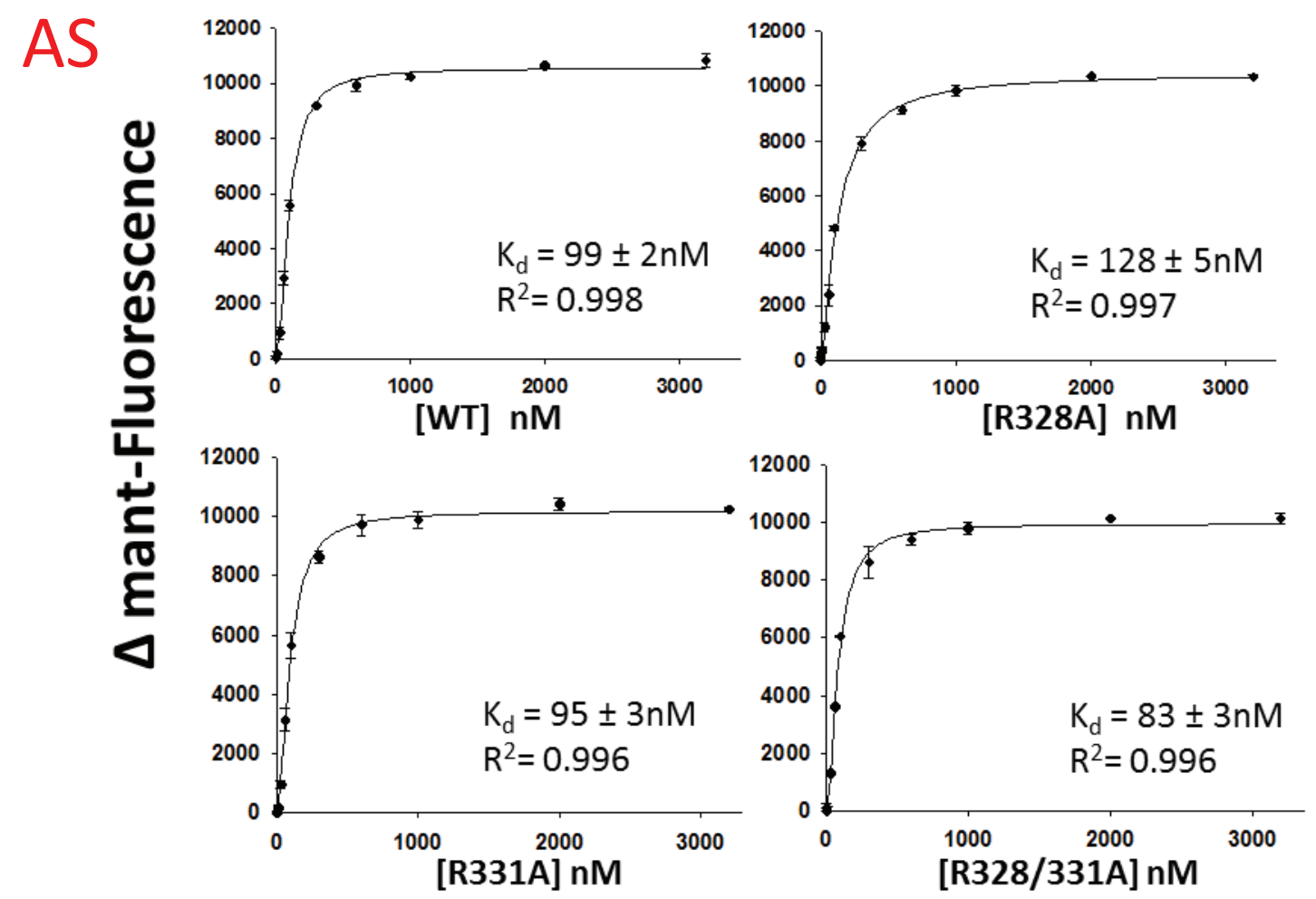

Figure 2.3. PAN's conserved arginines are not involved in regulating ATPbinding affinity. Equilibrium ATP-binding affinity was determined by monitoring the change in fluorescence intensity of mant-ATP $\gamma S(15 \mathrm{nM})$ in the presence of increasing amounts of WT PAN, PAN-R328A, PAN-R331A or PAN-R328/331A. The $\mathrm{X}$-axis is concentration of binding sites considering 2 high-affinity binding sites per PAN hexamer. The Michaelis-Menten binding hyperbola was fit to the raw data using non-linear regression analysis to obtain the $K_{d}$ (inset), the quality of fit $\left(R^{2}\right)$ is also shown. 
$200 \mu \mathrm{M}$, which produces the 4-bound state. All of the arginine mutants bound the same number of nucleotides as WT PAN showing (two- and four-bound states) (Fig. S2.4A). This indicates that the allosteries that regulated the nucleotide binding pattern were not perturbed by mutation of the conserved arginines. To assess if the "ortho" ATP-binding pattern required the conserved arginines we performed FRET as in Fig. 2.1 using the PAN-R328/331A mutant (Fig. 2.4b and Table 1). FRET analysis showed that the high affinity ATP-binding sites were the same distance from one another as WT PAN (Table 2.1). This experiment also establishes that the arginine mutation did not disrupt the quaternary structure of PAN. These combined ATP-binding analyses indicate that neither R328 nor R331 in PAN play any role in the allosteries that regulate the ATPbinding affinity, ATP-binding cooperativity, ATP stoichiometry, or the ATP-binding pattern.

\section{ATP-binding cooperativity in PAN does not require the conserved arginine residues}

To determine if cooperativity of ATP-binding is affected we performed an equilibrium ligand binding experiment with increasing amounts of m-ATPYS, using rapid spin columns to separate free from bound m-ATPyS. m-ATPYS bound to PAN with two different affinities (low and high) showing a biphasic curve as we have shown previously using ${ }^{35}$ S-ATPYS. The double arginine mutant showed the same binding curve as WT PAN (Fig. 2.4C-D), including a positive Hill slope for both curves. Furthermore, we observed positive cooperativity for WT PAN $(h=1.7+/-0.07)$ using a real-time ATPase assays (Fig. 2.4D). This Hill coefficient slightly less than two is thus consistent with ATPYS binding cooperativity for WT PAN and the arginine mutants as well as m-ATP- 


\section{a Number of nucleotides bound to PAN}

\begin{tabular}{|c|c|c|}
\hline & $10 \mu \mathrm{M}$ m-ATP $\gamma S$ & $200 \mu \mathrm{M}$ m-ATP $\gamma \mathrm{S}$ \\
\hline WT & $2.1 \pm 0.1$ & $3.7 \pm 0.5$ \\
\hline R328A & $1.8 \pm 0.3$ & $4.1 \pm 0.9$ \\
\hline R331A & $2.3 \pm 0.2$ & $3.8 \pm 0.3$ \\
\hline R328/331A & $1.8 \pm 0.1$ & $4.0 \pm 0.5$ \\
\hline
\end{tabular}

b

PAN- R328/331A)

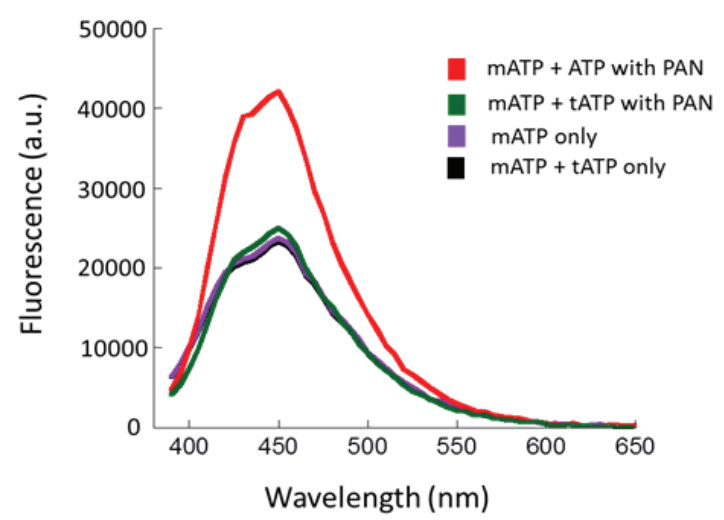

C
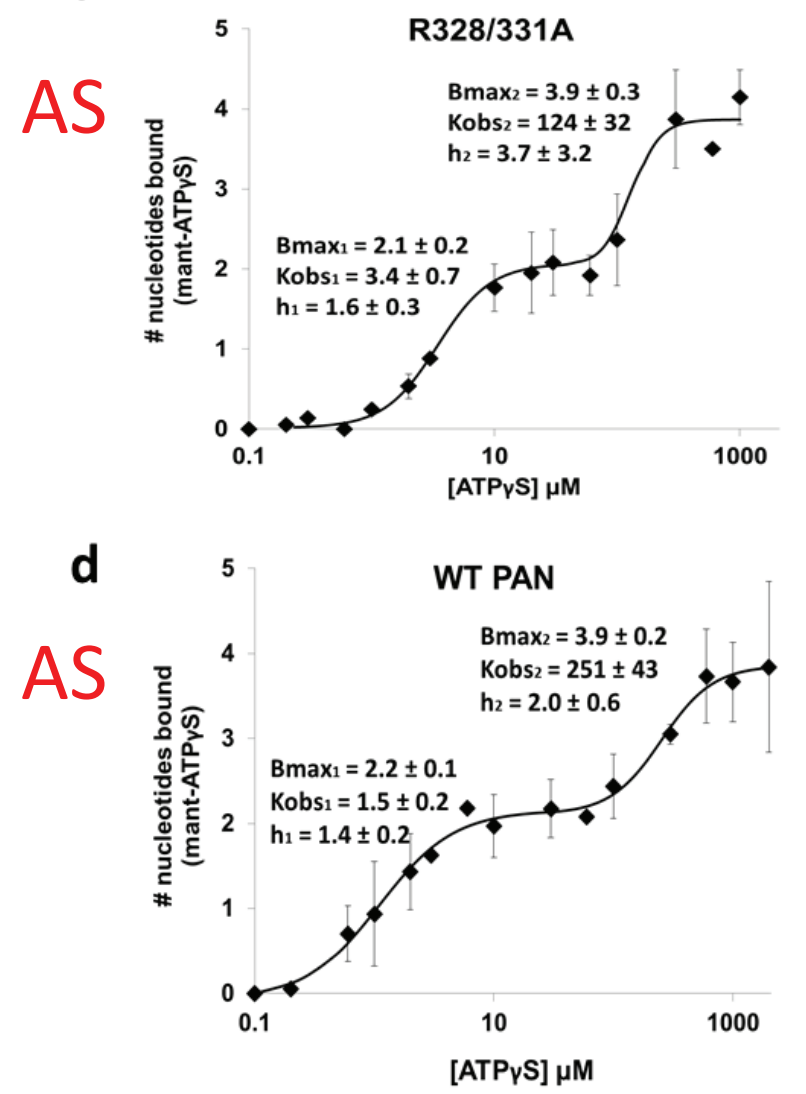

Figure 2.4. PAN's conserved arginines are not involved in regulating nucleotide stoichiometry, or ATP-binding pattern. A) The number of $m$ ATP $Y$ S bound to PAN (90nM) was determined by rapid separation of bound nucleotide from free nucleotide using $100 \mathrm{ml}$ spin columns at two different concentrations of ATP $\gamma$ S: 10 and $200 \mu \mathrm{M} .10 \mathrm{mM}$ saturates only the two high affinity sites, and $200 \mathrm{mM}$ allows near saturation of the high and low affinity sites (ATP and ADP sites; ref. 8). The number of bound nucleotides per PAN hexamer was calculated for WT and each arginine mutant as labeled. Data are means of 4 independent experiments +/- S.D. B) Emission spectra of m-ATP (as in Figure 1A but with PANR328/331A (1 $\mu \mathrm{M})$.

Quantifications are presented on Table 1. C\&D) The number of $\mathrm{m}$ ATPyS bound nucleotides to the labeled PAN variant was calculated as in (a) at increasing nucleotide concentrations to generate a binding curve. [PAN] was $200 \mathrm{nM}$ and thus the free ligand bind approximation is not met here and thus the $K$ value is expressed as Kobs as it does not accurately quantify affinity.

Representative data are presented from three independent experiments $+/-S D$. 
binding to PAN-E271Q. Importantly, the observed Hill coefficient in all three of these experiments is also consistent with an average of 2-bound nucleotides during operational conditions of ATP hydrolysis. These data demonstrate that neither R328 nor R331 are required for normal ATP-binding affinity, stoichiometry or the intersubunit communication that generates ATP-binding cooperativity.

\section{The conserved arginines are required for ATP-dependent substrate binding}

Since the ATP-binding affinity or binding pattern is not perturbed in the arginine mutants, we next asked if the functional effects of ATP-binding were altered. We first tested if ATP-dependent substrate binding to PAN is affected by the mutation. We monitored binding of GFP-ssrA to PAN using fluorescence polarization (FP). The unstructured ssrA tag allows binding to pore-1 loops in PAN and $\mathrm{ClpX}^{13,40}$. The binding ATPYS to WT PAN (but not ADP) polarized GFP-ssrA by $17 \mathrm{mP}$ (Fig. 2.5A). This demonstrates ATP-binding to PAN is required to trigger GFP-ssrA association, which we have also shown previously ${ }^{8}$. However, none of the arginine mutants could polarize GFP-ssrA in the presence of ATPYS. This indicates that both of the conserved arginines are necessary for ATP-dependent triggering of substrate binding. Therefore, these trans-arginines appear to detect the ATP-bound state of the neighboring subunit and trigger allosteric conformational changes that allow substrate binding. For further verification we tested the substrate unfolding activity of PAN in the presence of ATP and the substrate stimulation activity. The PAN mutants could not unfold GFP-ssrA to any extent (Fig. S2.5A-B) and their ATPase activity was not stimulated by GFP-ssrA (Fig. S2.5C). These is consistent with a loss of substrate binding ability in the mutants. 
a

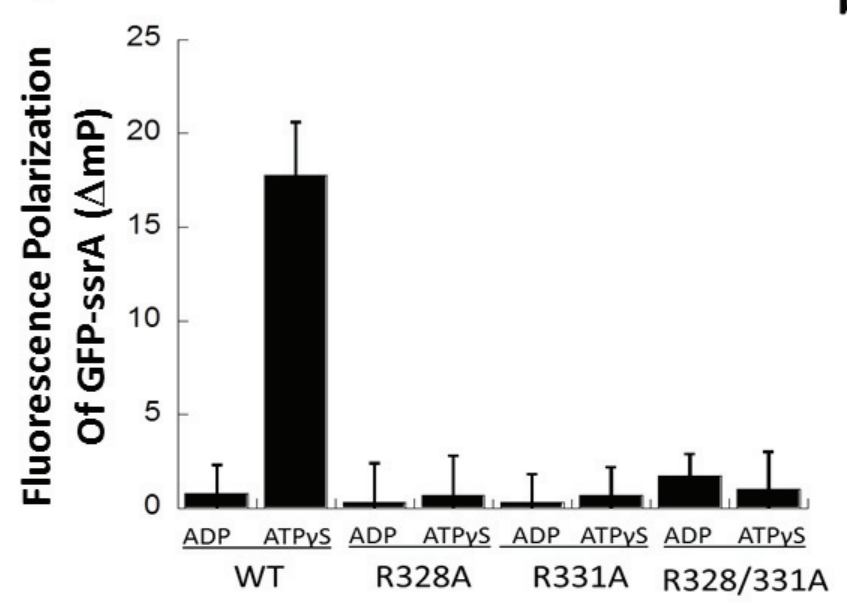

b

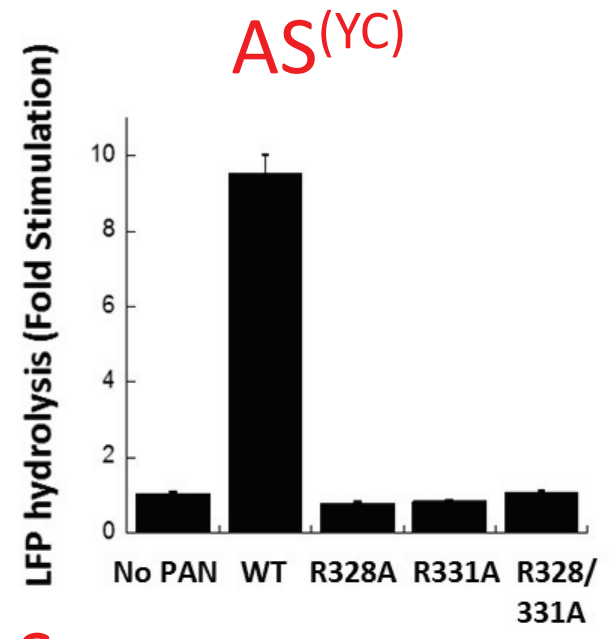

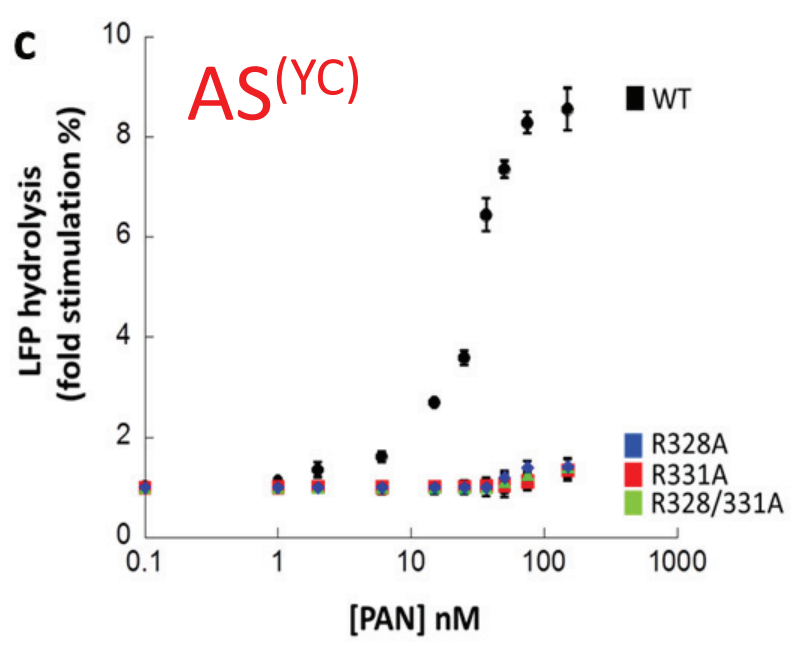

d $A S$

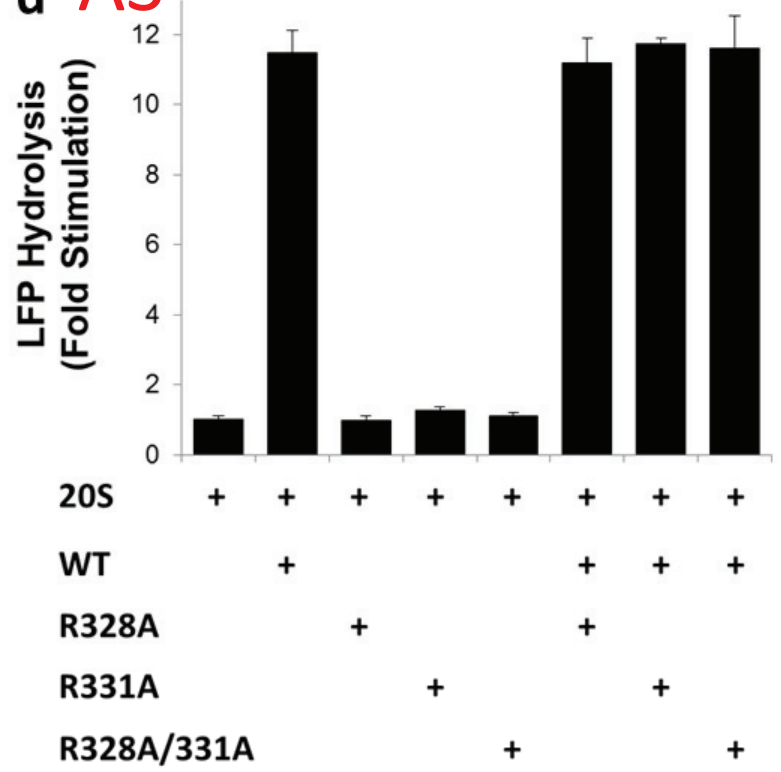

Figure 2.5. Mutation of either one of PAN's conserved arginines abrogates ATPdependent substrate binding and $20 \mathrm{~S}$ gate-opening. A) Fluorescence polarization was used to monitor the binding of GFP-ssrA $(0.08 \mu \mathrm{M})$ to PAN $(0.12 \mu \mathrm{M})$ or its arginine mutants in the presence of $1 \mathrm{mM} \mathrm{ADP}$ (negative control) or $1 \mathrm{mM}$ ATPYS. B) Gate-opening in the $20 \mathrm{~S}$ proteasome $(20 \mathrm{nM})$ by PAN WT, or its mutants $(80 \mathrm{nM})$, was monitored with the LFP peptide hydrolysis in the presence of $10 \mu \mathrm{M}$ ATPYS. "No PAN" is 20S (archaeal) alone. C) Gate-opening in the $20 \mathrm{~S}$ proteasome $(20 \mathrm{nM})$ as a function of increasing concentration of WT PAN and arginine mutants. D) Gate opening assay by the WT PAN (10nM) as in (b) but also in the presence of the other indicated PAN mutants $(10 \mathrm{nM})$ to determine if the mutants can compete with WT for binding to the 20S. All data are representative experiments and are the means of three independent measurements \pm S.D. 
The conserved arginines are required for ATPase-20S association and $20 \mathrm{~S}$ gate opening

Besides substrate binding, another important effect of ATP-binding is the activation of PAN-20S association and gate-opening ${ }^{11,17}$. The addition of ATPYS to WT PAN and 20 s stimulated hydrolysis of the internally quenched LFP peptide $\sim 9$-fold, demonstrating PAN-20S association and gate-opening (Fig. 2.5B). In contrast, none of the mutants could stimulate LFP hydrolysis (Fig. 2.5B). To ensure the arginine mutations did not simply alter the affinity of PAN for the $20 \mathrm{~S}$, we titrated PAN on the $20 \mathrm{~S}$ to saturating concentrations. Only WT PAN showed saturation of the $20 \mathrm{~S}$ (Fig. 2.5C). It's also plausible that the PAN mutants could bind the $20 \mathrm{~S}$ without actually inducing gateopening. To test for this possibility we performed a competition experiment between the mutants and WT PAN. None of the mutants were able to inhibit WT PAN gate-opening (Fig. 2.5D) indicating that the arginine mutants could not bind the 20S. These results indicate that although the arginine mutants bind to ATP with the same affinity and pattern as WT PAN, they could not bind to the $20 \mathrm{~S}$ proteasome to induce gate opening. Therefore, both of the trans arginines are required to allow ATP-binding to allosterically communicate the ATP bound state to the C-terminal HbYX motif to trigger PAN-20S association and $20 \mathrm{~S}$ gate-opening.

The conserved arginines do not play a role in the dissociation kinetics of ADP The role these two trans arginines play in catalysis of ATP hydrolysis has not been investigated for the proteasomal ATPases. At any individual active site, the ATP hydrolysis cycle goes through 3 primary steps: 1) ATP-binding, 2) formation of the transition state and cleavage of the gamma phosphate (and its release), and 3) ADP 
dissociation, which is required to allow binding of ATP in the next round of catalysis. ADP dissociation is thought to be the rate-limiting step in this process. Since we already know that step one, ATP-binding, is not affected by arginine mutation (Fig. 2.2, 2.3 and Table 2.1), either step 2 or 3 must be perturbed to cause the observed loss of ATPase activity. To determine which step is impaired, we performed a stopped-flow experiment to determine if the off rate of ADP was affected by mutation of the arginine residues. Equimolar amounts of PAN (150 nM) was preloaded with m-ADP $(150 \mathrm{nM})$ in the first injection syringe. These concentrations allowed for $\sim 80 \%$ of the m-ADP to be bound. The second syringe contained the same buffer with saturating amounts of ADP (2 mM). The two samples were injected together pneumatically. Mant fluorescence was monitored every $100 \mathrm{mS}$ during the competition experiment, and the raw data were fit to single or double exponential decay curves. A single exponential decay curve did not fit any of the generated curves, but a double exponential curve fit well with appropriate residuals (Fig. 2.6A). These fits suggest that PAN contains two different types of sites for bound ADP, each with a slightly different off rate. Since PAN is equimolar with mADP, it's expected that most of the PAN has a single m-ADP bound while some contained two bound m-ADPs, the presence of these two different bound populations could explain why two different decay rates are observed, but other explanations are possible. Mutation of conserved arginines has little to no effect on the rate of ADP dissociation, especially for the fast step (Fig. 2.6B). In fact, mutation of both arginines actually increased the fast and slow off rate by $11 \%$ and $32 \%$, respectively and the change in the fast rate was not statistically significant. Therefore, the complete loss of ATPase activity caused by mutation of the conserved arginines is expected to be due to 
a
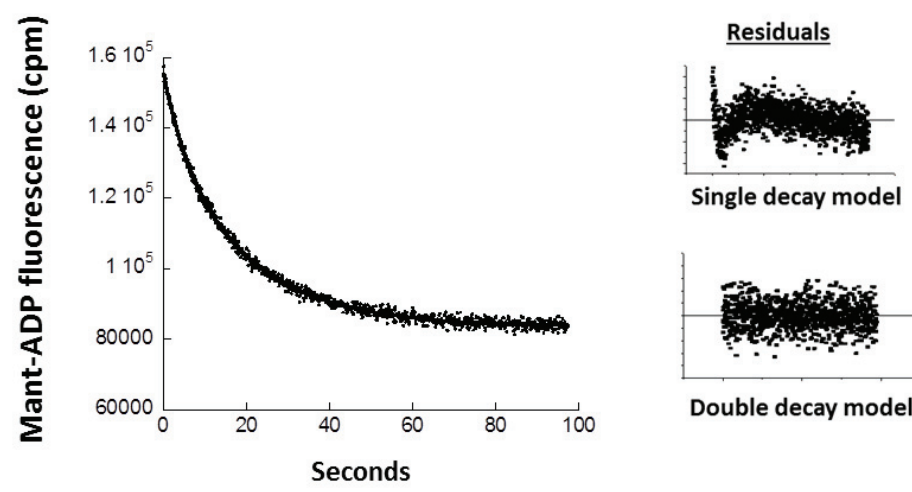

b

\begin{tabular}{|c|c|c|}
\hline & $\begin{array}{c}\mathbf{T}_{\mathbf{1 / 2}} \text { (fast) } \\
\text { (sec) }\end{array}$ & $\begin{array}{c}\mathbf{T}_{\mathbf{1 / 2}} \text { (slow) } \\
\text { (sec) }\end{array}$ \\
\hline WT & $3.74 \pm 0.42$ & $13.99 \pm 0.47$ \\
\hline R328A & $2.69 \pm 0.92$ & $8.60 \pm 0.35$ \\
\hline R331A & $2.92 \pm 0.84$ & $9.93 \pm 1.61$ \\
\hline R328/331A & $3.32 \pm 1.16$ & $9.51 \pm 0.58$ \\
\hline
\end{tabular}

C

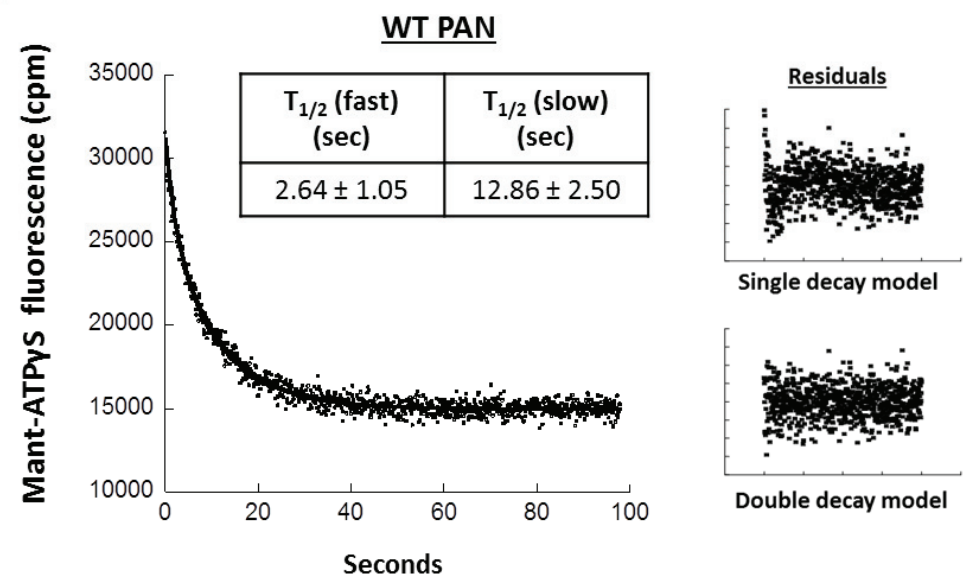

Figure 2.6. The ATP and ADP off rates are similar, and the ADP off rate is not affected by mutation of the arginine finger. A) Pre-steady state dissociation of the prebound m-ADP (150 nM) from WT PAN (150 nM) was monitored by stopped-flow at $37^{\circ} \mathrm{C}$. Saturating amounts ADP $(2 \mathrm{mM})$ were used to compete off the $\mathrm{m}$-ADP. The residuals from fitting the raw data with single or double exponential decay models are shown (right). B) The half-life ( $\left.T_{1 / 2}\right)$ of the bound $m$-ADP to WT PAN and the arginine mutants for the double decay model is presented, showing both fast and slow rates. C) Pre-steady state dissociation of prebound m-ATPyS $(1 \mu \mathrm{M})$ from WT PAN $(0.5 \mu \mathrm{M})$ was monitored as in A. Saturating amounts ADP (4 mM) were used to compete off the m-ATPyS. Residuals for the single and double decay models are shown (right). The determined half-life for both fast and slow rates for m-ATPYS are shown in the inset (double decay model). 
a loss in the ability to stabilize the transition state that catalyzes $\gamma$-phosphate cleavage (i.e., step 2 is defective), rather than due to a decreased ADP off rate.

\section{ATP-binding is in equilibrium during normal ATP hydrolysis-the ATP off rate is similar to the ADP off rate}

In order to probe the process of ATP hydrolysis around the proteasomal ATPase ring, some groups have individually deactivated some but not all of the subunits in an ATPase ring ${ }^{15,41,42}$. Interestingly, a mutation of only a single subunit (1 of 6 ) has only a minor impact on the rates of ATP hydrolysis when substrate is not bound ${ }^{15,41}$. This suggests that either: 1) some subunits in the WT ring never bind and hydrolyze ATP (i.e., there is no subunit switching and thus the inactivation of inactive subunits doesn't impact rates of hydrolysis), or 2) that all subunits can hydrolyze ATP, but deactivated subunits can be skipped or partially ignored. The former explanation has been convincingly ruled out, at least in studies of ClpX, where subunit conformational switching was shown to occur ${ }^{28,43}$. Additionally, the existence of multiple ring conformations in the 26S ATPases also suggests subunit conformational switching in the proteasomal ATPases. The later explanation (\#2) suggests that strictly sequential mechanisms of ATP hydrolysis are not possible and the Sauer group has proposed that a probabilistic model can explain this subunit skipping phenomenon ${ }^{29,43}$. The probabilistic model indicates that any subunit (with a bound ATP) can bind and hydrolyze ATP, but that some subunits can have a higher probability of binding or firing than others presumably depending on which subunits are currently bound to ATP. However, our data indicates that ATP-binding is highly ordered and controlled, at least in the proteasomal ATPases, suggesting a strictly sequential mechanism of ATP 
hydrolysis. But how can a sequential mechanism be rectified with subunit skipping? To answer this question we sought to determine if ATP can leave PAN without being hydrolyzed; if so this indicates that ATP could bind to new subunits, resetting their position in the ring. If this could occur on a time scale that is relevant to ATP hydrolysis rates, then this could explain how subunit skipping could occur in a mechanistically sequentially functioning ATPase with minimal impact on the rates of ATP hydrolysis. To determine this we performed a stopped-flow experiment similar to Fig. 2.6A, but this time we prebound m-ATPYS to WT PAN in one syringe, and added saturating amounts of competing ADP (4 mM) in the other syringe. ADP was used as a competitor rather than ATPYS, because the competitor will bind to the low affinity sites (ADP sites) first, which has no observable effect on PAN function ${ }^{17}$, before competing at the high affinity sites. Alternatively, if ATPYS was used as a competitor it would induce a strained 4bound ATP state, which may alter the normal off rates. Again a single exponential fit did not produce satisfactory residuals but a double exponential decay fit well (Fig. 2.6C). The fast off rate was 2.6 seconds, which was slightly faster but comparable to the off rate for ADP from WT PAN. The slow off rate was $13 \mathrm{sec}$, also similar to the slow ADP off rate. Thus, ATP has a similar off rate as ADP. Since the ADP off rate is the rate limiting step (i.e., new ATPs cannot bind and be hydrolyzed until ADP leaves), this suggests that approximately half of the ATP that binds to PAN leaves without being hydrolyzed. This indicates that ATP-binding is in thermodynamic equilibrium (coming and going) during the ATP hydrolysis cycle to such an extent that ring resetting is likely to occur between ATP hydrolysis events during normal operation. The impact of thermodynamic ring resetting would also be expected to be greater on an enzyme that 
exhibits dwell/burst kinetics as has been proposed for $\mathrm{ClpX}^{44}$, due to lengthy pauses in the cycle. Therefore, these results demonstrate that thermodynamic ring resetting can explain how subunit skipping can occur in a strictly sequential ATPase, especially when ATP hydrolysis is perturbed (or delayed) in single subunit mutants. 


\section{Discussion}

Here we've shown that PAN has two conserved arginines that are both required for ATP hydrolysis, confirming their expected, but unverified, role as an arginine finger. But why do some of the AAA ATPases have two arginines in this position rather than just one, when one arginine is sufficient for catalysis in many different types of oligomeric nucleotidases ${ }^{30,34}$ ? One possibility is that this dual arginine finger arrangement is more efficient at sterically transmitting allosteric changes—due to ATP-binding—between neighboring subunits. Here we found that the proteasome requires this dual arginine finger to trigger two specific ATP-binding dependent functions: 1) for triggering substrate binding and 2) for inducing $\mathrm{HbYX}$ dependent PAN-20S association coupled to $20 \mathrm{~S}$ gateopening (Fig. 2.7A). This dual arginine finger in the proteasomal ATPase PAN thus performs two independent functions: 1) to catalyze ATP hydrolysis, and 2) to allosterically regulate mechanistically-critical conformational changes.

Because the arginine finger in the proteasomal ATPases structurally functions "in trans" (PDB:4BGR), this indicates that the allosteric effects are carried out by the subunit that contains the arginine finger (Arginine subunit) and not the one that is bound to the ATP (Walker subunit), since ATP can bind to the Walker A/B motif but cannot trigger ATP-binding effects without both arginines being present (Fig. 2.7B). One explanation is that the $\mathrm{OB}$ domain promotes hexamerization (all mutants run as hexamer by native gel) while the arginine mutation disrupts the ATPase domain interaction leading to their separation. However, this possibility is ruled out since the ATP-binding sites are positioned at exactly the same distance in WT and the double 

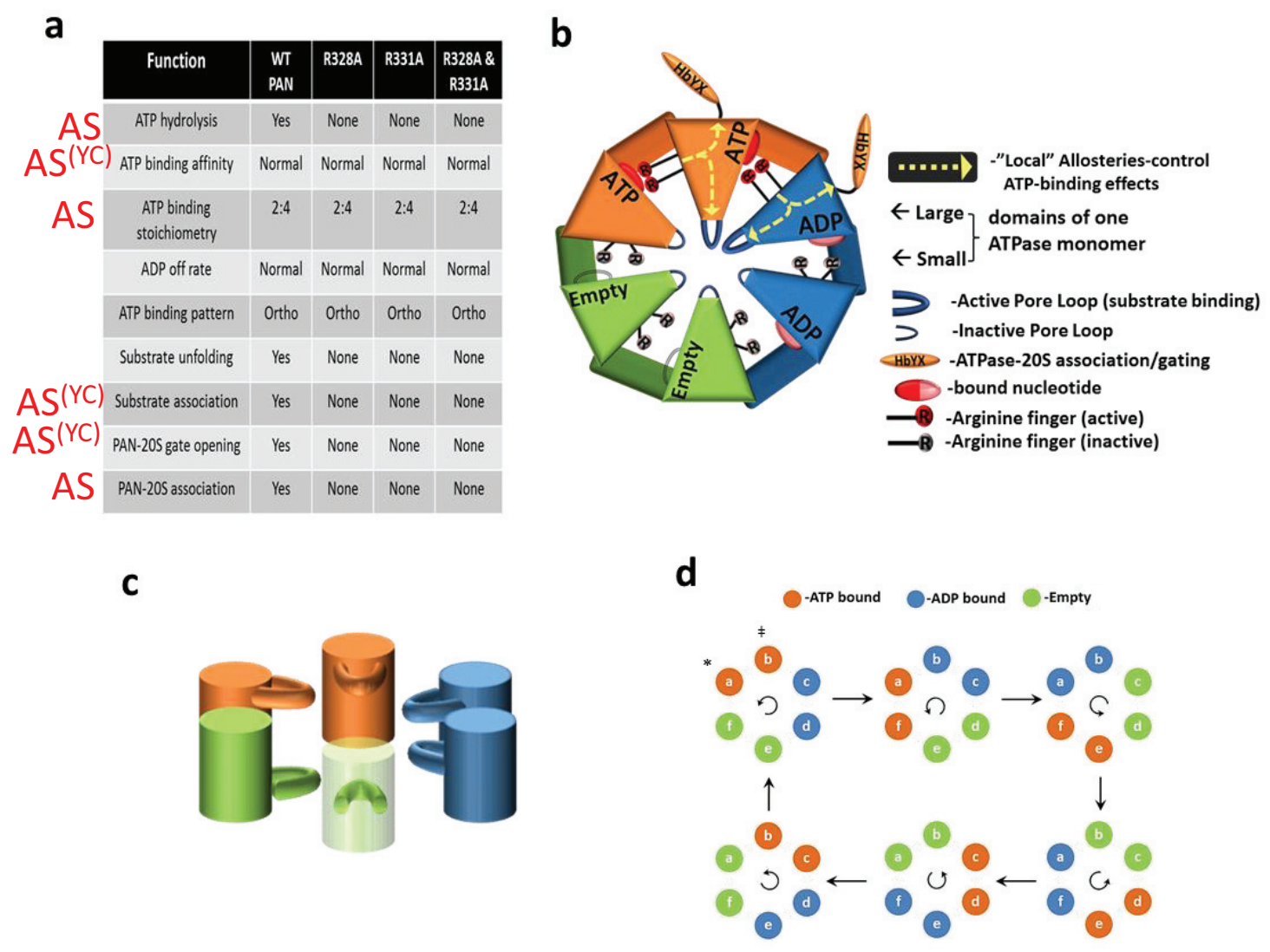

Figure 2.7. Allosteric model of nucleotide binding and exchange in the proteasomal ATPases and its implications on the trans-mechanism of substrate translocation. A) Summary of the roles that conserved arginines play in mediating ATP-binding effects in the proteasomal ATPase PAN. B) Ortho ATP-binding model for the proteasomal ATPase PAN, and the local allosteries that are mediated by the conserved arginine finger residues (yellow arrows), which control the depicted ATPbinding effects. The trans-functioning arginine finger (requiring both conserved arginines) contacts ATP in its counterclockwise neighbor (Walker subunit), this allosterically triggers substrate-binding and $\mathrm{HbYX}$ exposure in its own subunit (Arginine subunit). The ortho nucleotide binding pattern is controlled by the global conformation of the ATPase ring, in coordination with the helical topology and is not controlled by the local allosteries of the arginine fingers. C) A model depicting nucleotide bound lockwasher-like topology of 26S ATPases after substrate engagement. Color coding of this helical topology would correspond to the color coding in B to depict rotation of the helical conformation with subunit progression. D) Model of ATP hydrolysis subunit progression undergoing one complete cycle. Individual subunits (a-f) remain fixed as ATP is hydrolyzed with a single subunit progression around the ring starting with the lagging ATP-bound subunit. The nucleotide bound to each subunit is indicated in the key by color. The leading $\left(^{*}\right)$ and lagging ( $\neq)$ subunits are indicated in the first iteration (top-left). The top-left and bottom-right configurations in this cycle could correspond to the two resting states of the 26S ATPases observed in cryo-EM studies mentioned in the discussion (with either Rpt1 or Rpt3 at the top positions). 


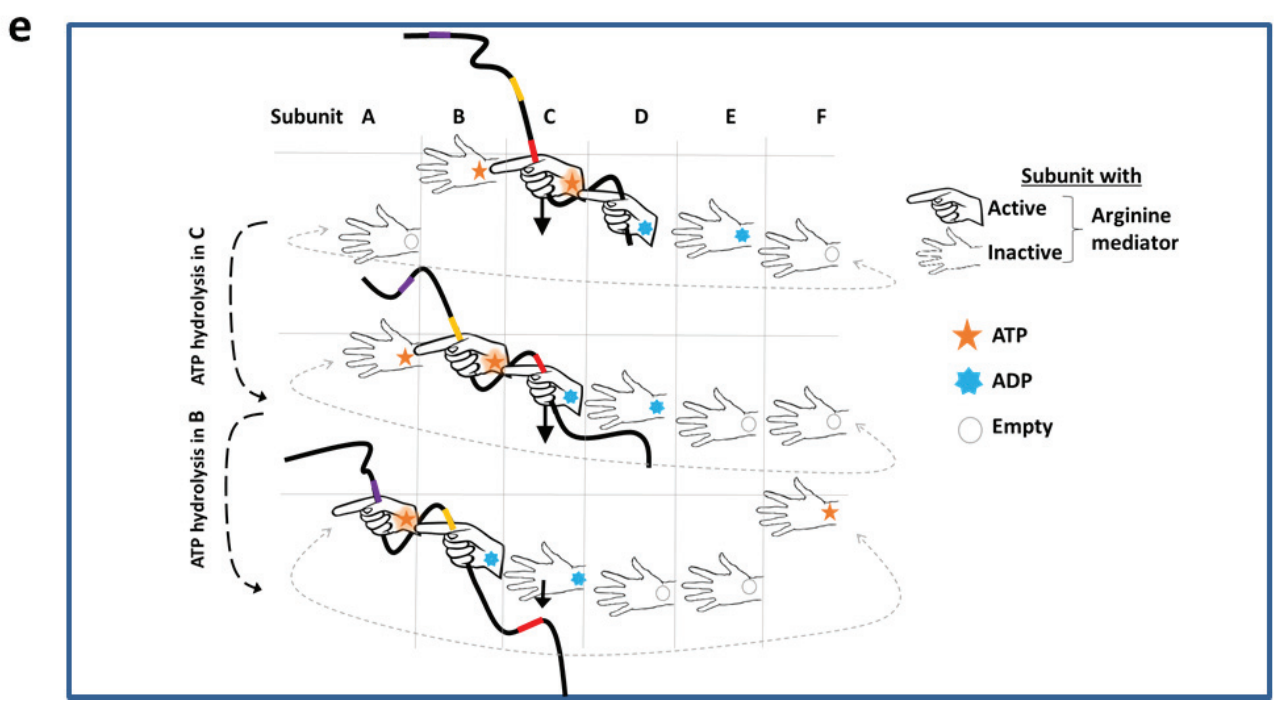

Figure 2.7. (Continued). E) Model depicting protein translocation in proteasomal ATPases. The ortho ATP-binding pattern and the local allosteries of the trans arginine finger are combined to demonstrate how ATP-binding and hydrolysis could result in translocation of engaged substrate. Here the six subunits of the ATPase ring are peeled open and the height of the pore loop (hand) is indicated by vertical position. The closed hand with finger indicates a subunit whose arginine finger is contacting its neighbor's bound ATP, and thus has affinity for substrate. Therefore, ATP hydrolysis in this subunit results in movement of the substrate downward without losing its "grip" on the substrate, at which time the next subunit can also bind after ATP binds to its neighbor. In this way the substrate is never released until it translocation is completed, thus generating a mechanism for highly efficient unidirectional translocation into the $20 \mathrm{~S}$ proteasome. 
mutant (Fig. 2.4B), implying the quaternary structure of the mutants is intact. Therefore, ATP-binding in the Walker subunit must trigger substrate binding and $\mathrm{HbYX}$ exposure (allowing binding to the 20S) in the neighboring Arginine subunit. This of course assumes that the arginine finger acts "locally"-meaning it only affects the conformation of its own subunit (Fig. 2.7B). Alternatively, the arginine finger could mediate "global" conformational changes-meaning that it could affect the conformation of all, or most, of the subunits in the ring to elicit these effects. However, mutation of these conserved arginines does not disrupt: 1) ATP-binding affinity, ATP-binding cooperativity, or the pattern of ATP-binding, which requires global allosteric control-since all six subunits are involved (i.e., 2-high, 2-low and 2-no affinity subunits at saturation; Fig. 2.3, 2.4A, 2.4C, and ref. 8). Such global control of ATP-binding is also consistent with a recent study of ClpX, which showed that large/small domain contacts between neighboring subunits controlled a rigid-body motion for global conformational changes of the ClpX ATPase ring ${ }^{28,45}$. These results therefore demonstrate that the function of the dual arginine finger can be decoupled from the global allosteries that regulate ATP-binding kinetics and the ATP-binding pattern. Therefore, the effects are necessarily limited to individual subunits (or between a subunit and its neighbor), which we consider a "local" effect. In contrast, a study of the arginine finger in covalently linked ClpB oligomers indicated that it was involved in ATP-binding kinetics and was required for the allosteries that regulate ATP-binding cooperativity ${ }^{46}$, which we do not observe for PAN. However, a crystal structure of the homohexameric AAA+ ATPase NtrC1 showed a PAN analogous "local" acting mechanism for its arginine finger, whereby the arginine, upon contacting the neighbor-bound ATP, induces a local conformational change in a 
surface loop that triggers substrate $(\sigma 54)$ binding $^{47}$. Our data implies that substrate binding to PAN and HbYX-20S association occurs in the Arginine subunit and not the Walker subunit, as we and others assumed previously ${ }^{8,41}$. Because substrate binding to the Arginine subunit is critical for the mechanochemical coupling that drives protein translocation, the organization of ATP-binding and hydrolysis pattern must be critical to understanding the mechanisms of the proteasomal ATPases (see below).

The FRET experiments presented here show that PAN and the 26S ATPases bind to ATP at apparently only neighboring subunits (Ortho-positioned, Fig. 2.1), and prior experiments showed that they both have similar types of high and low affinity subunits. Together these results demonstrate that PAN and the 26S ATPases share a similar "global" allosteric system that regulates the nucleotide binding pattern. In addition, while the distance between the low affinity sites (ADP-sites) could not be empirically determined with FRET, we can logically conclude that once both ortho bound ATPs are hydrolyzed they will become ortho bound ADPs. It's important to note here that several recent cryo-EM structures of the $26 \mathrm{~S}$ proteasome show that its ATPases have a right-handed helical staircase topology (defined by the vertical position of the substrate-translocating pore loops) $)^{21,48}$, which is complementary with the "ortho" ATP-binding pattern (Fig. 2.7B). This helical topology is observed in both the apo state with $\mathrm{ATP}_{\mathrm{h}}{ }^{21,48}$ and in the translocation competent state (i.e., with either substrate ${ }^{7}$ ) or ATPYS bound ${ }^{5}$. A three subunit rotation of the helical topology (a conformational transition) is required to convert between these two helical states. Because ATP-binding and hydrolysis must drive the conformational changes that produce work, the 
conformation of the individual subunits around the ring must be tightly linked to their bound nucleotide. It's expected then that rotation of the helical topology with substrate bound to the proper pore loop(s) would result in substrate translocation through the ATPase ring. Because the nucleotide binding pattern, as well as the helical topology appear to be regulated globally (and mirror one another to some extent), we postulate that the helical topology regulates the nucleotide binding configuration. So how might the "ortho" binding pattern best fit the topology of the known helical states? Since unidirectional translocation (into the 20S) requires that substrates bind near the top of the ATPase ring ( $\mathrm{N}$-terminal side) then be moved downward into the pore upon ATP hydrolysis ${ }^{41}$, we expect that the two highest positioned subunits are ATP-bound, since substrate binding requires the ATP-bound state. Based on this, we place the ATPbound subunits at the top of the lockwasher topology in our working model (Fig. 2.7C). In addition, ATP hydrolysis to ADP must generate work on the substrate by translocating it ${ }^{10,49}$, and thus the subunits with pore loops vertically lower in the ring should be the ADP-bound subunits. For model building purposes we place the pair of ADP subunits clockwise to the ATP subunits rather than counterclockwise (Fig. 2.7B-C) and it will become evident below that this arrangement combined with our new understanding of the arginine mediator allows the most coherent model for translocation.

There are two possible mechanisms for the progression of ATP hydrolysis with an ortho ATP-binding pattern, 1) whereby concerted pairs of subunits hydrolyze ATP and sequentially progress around the ring (paired progression), or 2) where ATP binds 
to two subunits but only one of them hydrolyzes it's ATP at a time with sequential progression around the ring (single subunit progression). Since only ATP can stimulate the binding of the substrates GFP-ssrA or FITC-casein ${ }^{8}$, at least one bound ATP is required to maintain PAN in the substrate bound state. Therefore, if PAN hydrolyzed both of its ATPs to ADPs in a paired progression, it will lose its affinity for the substrate, which would allow the substrate to slip out of the pore, especially since diffusion is quite fast compared to the rates of ATP hydrolysis $(\sim 1 / \mathrm{sec})$. In contrast, single subunit progression would allow at least one subunit to be in the ATP-bound state at all times, implying that substrate would remain bound to the pore loop during ATP hydrolysis. Thus, single subunit progression supports a far more plausible and efficient mechanism to power protein unfolding. So would it matter which of the two "ortho" bound ATPs hydrolyze first? The only way the "ortho" binding pattern can be maintained throughout a firing cycle (which is most consistent with our FRET measurements) is if the lagging (clockwise) ATP is hydrolyzed first (Fig. 2.7D). This is the simplest model for conformational progression of "ortho" bound subunits and would also be allosterically favored since our data shows a positive cooperativity between the "ortho" ATP subunits allowing for a forward moving chain reaction of ATP-binding and hydrolysis that can propagate around the ring. The directionality for subunit progression we have built into our working model is based on the counterclockwise directionality of the arginine finger, as it is an important mediator of mechanochemical coupling in PAN. Because the arginine finger is required for substrate binding, ATP-binding to the Walker subunit must trigger substrate binding in its clockwise Arginine subunit (see Fig. 2.7B). For efficient translocation to occur the substrate bound subunit must maintain its substrate bound 
state in both the ATP and ADP bound configurations. This would allow the subunit to hydrolyze ATP to ADP and to do work on the substrate by moving it downward. This aspect is similar to the proposed model of the m-AAA protease's requirement for maintaining ATP-binding for substrate gripping while processing a substrate ${ }^{50}$. Only hydrolysis in the lagging ATP-bound subunit would allow for maintenance of the substrate bound state during ATP hydrolysis, since its arginine finger remains engaged with ATP in the Arginine subunit (Fig. 2.7B\&D). Based on this logic, the 2 ADP subunits must be clockwise neighbors from the ATP bound subunits. Thus, in this model, ATP would bind to the lagging empty subunits after the lagging ADP leaves (because two subunits are always empty), progressing the helical topology by a single subunit and translocating the substrate by a single step. This indicates that the progression of ATP hydrolysis around the ATPase ring, mirroring the rotating helical topology, would be counterclockwise (Fig. 7C and 7D).

Our findings demonstrating the trans-acting nature of the arginine finger as well as the ortho ATP-binding configuration illuminate an important and perhaps underappreciated necessity for efficient protein translocation through a pore. To visualize progression of the lockwasher topology and how this relates to the function of the Arginine and Walker subunits, we have generated a translocation cartoon model (Fig. 2.7E) that is based on the culmination of these findings and discussion. This model incorporates the "ortho" ATP-binding pattern and helical topology with the arginine finger along with single subunit progression to depict how these elements combine to generate a surprisingly simple working model for protein translocation. The 
hydrolyzing Arginine subunit (Fig. 2.7E, Subunit-C near top) can only maintain "grip" on the substrate if the Walker subunit (Subunit-B top) remains in the ATP-bound state. Only the single subunit progression of the "ortho" ATP-binding pattern, combined with the "trans" allosteric effects of the arginine finger on substrate binding, can support this mechanism. We imagine that ATP hydrolysis (i.e., conversion to the ADP bound state) promotes global conformational rotation of the helical topology by one subunit pulling the substrate downward. The Subunit-D would then release the substrate after ATP hydrolysis since its arginine finger would no longer be engaged with ATP. Cooperative ATP-binding to the empty Subunit-A would restart the cycle and keep the helical topology moving in a counterclockwise direction. This proposed model for protein translocation and the associated ATP-binding/exchange model are consistent with the function of a local acting arginine finger that functions "in trans", the "ortho" nucleotide binding pattern, the observed positive cooperativity, and the lockwasher-like conformational arrangements of the 26S ATPase subunits.

The concentration of ATP in the cell is greater than ten times the binding affinity of the low affinity ATP-binding sites, implying that it is possible for PAN to exist in a 4bound state ${ }^{8}$. However, our data suggests that PAN normally only binds to 2-ATPs during hydrolysis. What evidence supports this? 1) The Hill coefficient for ATP is approaching $2(\mathrm{~h}=1.7+/-0.07), 2)$ presence of 2-high, 2-low, and 2 no affinity subunits, 3) the saturated 4-bound ATP state functions sub-optimally compared to the 2-bound state (Fig. S2.3A and Fig. 2.4) ADP leaving is thought to be rate limiting. The fourth point is important because PAN cannot bind more than 4 nucleotides. Therefore, even 
at cellular saturating conditions, for a new ATP to bind it must first wait for ADP to leave. This implies that PAN normally hydrolyzes ATP while ADP is bound and thus a 4-bound ATP state would not exist even at saturating ATP. In addition, if a four bound state were to exist, then we would predict that four of the six pore loops would be bound to substrate simultaneously. If this were the case, then rapid hydrolysis in all four subunits would only result in translocation by a single step (e.g., in the game of tug-of-war, when four people on one side of the rope take one step back, the rope only translates by one step). Such a mechanism seems inefficient, and doesn't agree with single molecule force experiments of ClpX, which show that it can take 1-4 nm translocation steps, which are interpreted as 1-4 subunit bursts of ATP hydrolysis from a 4-ATP bound state $^{44,51}$. An alternative interpretation is to assume that new ATP could occasionally bind during the step burst (especially at saturating ATP) allowing for longer than $2 \mathrm{~nm}$ steps, while never binding more than 2 ATPs at a time, which is expected for single subunit progression. However, PAN and ClpX are quite different AAA ATPases, (e.g., ClpX has a cis-functioning Sensor 2 arginine, which mediates substrate and ClpP binding, instead of a functional trans arginine finger) and no single molecule data is yet available for PAN or the 26S ATPases, so further studies are needed to make these comparisons.

Most AAA+ ATPases show a high level of coordination between the subunits and taking into account the strict allosteric constraints of nucleotide binding, it would be predicted that the proteasomal ATPases function by an ordered sequential mechanism. However, studies of the $26 \mathrm{~S}$ proteasome ${ }^{15,41}$ and $\mathrm{ClpX}^{28,29,44,51}$ with various 
combinations of deactivated subunits have suggested that subunit skipping can occur. A partial probabilistic model of ATP hydrolysis with a degree of intersubunit coordination was developed for ClpX to explain such results ${ }^{28,29,44,51}$. However, our FRET measurements indicate that ATP only binds to neighboring subunits, which is not consistent with a probabilistic model and instead supports a strictly sequential mechanism of ATP hydrolysis. How do we rectify these observations with the data presented here? It's well understood that ligand binding is a thermodynamic equilibrium process and we've shown here that the off rates $(2.6 \mathrm{sec})$ for ATP are comparable to the catalytic rates $(1 \mathrm{ATP} / \mathrm{sec} /$ hexamer; Fig. $2.6 \mathrm{C})$. Thus, any one subunit in the hexamer hydrolyzes ATP every 6 seconds (on average), but after binding ATP it leaves after $2.6 \mathrm{sec}$ (on average) if it's not hydrolyzed. This indicates that when a mutated subunit is reached in a sequential cycle only seconds, on average, have to pass before ATP leaves to allow new ATP to bind to a new site, thus resetting the ring and allowing for continuation of the sequential cycle. In this sense ring resetting can be expected simply based on the thermodynamics of nucleotide binding, and thus its relevance may only be evident when mutations are introduced that impair normal function. While the extent of such thermodynamic ring resetting is unknown during normal operation, if such resetting occurs frequently in a WT enzyme, then we expect that a probabilistic model would be needed to describe function. However, if it is a rare event, then a sequential model would be sufficient to describe the inherent operations of the ATPase ring. In other words, we hypothesize that these ATPases function sequentially between thermodynamic ring resetting events when they occur. Based on these data and rationale it is our model that the proteasomal ATPases function by a sequential 
mechanism that is generated by the inherent global allosteries of the multisubunit complex, but like all enzymes its mechanism is subject to standard thermodynamic considerations. 


\section{Methods}

Materials, Protein Expression and Purification - PAN, GFP-ssrA, T20S, and LFP were prepared as described ${ }^{10,17}$. Expression vectors for the PAN arginine mutants (R328A, R331A, and R328/331A) in pRSETA were generated by site-directed mutagenesis and were confirmed by sequencing. The purest available forms of ATP, ATPYS and ADP were purchased from Sigma and were stored at $-80^{\circ} \mathrm{C}$ until use. Mant-ATPYS and Mant-ADP were purchased from Jena Bioscience. Mant-ATP and TNP-ATP were purchased from Molecular Probes. Bovine 26S proteasome was purified by previously described UBL-UIM method ${ }^{52}$ and exchanged with reaction buffer by rapid spin column or by dialysis (4hr) immediately prior to use.

$20 S$ Gate Opening, unfolding and protein degradation - Enzymatic reactions with archaeal proteasomes and ATPase complexes were performed at $45^{\circ} \mathrm{C}$ as described previously ${ }^{10}$. To measure $20 S$ gate opening, the internally quenched fluorogenic peptide substrate (LFP) was dissolved in DMSO and used at a final concentration of $10 \mu \mathrm{M}$ in the presence of the indicated nucleotide (ATPYS). GFP-ssrA substrate unfolding and degradation was monitored fluorometrically as previously described ${ }^{10}$.

Steady-state nucleotide binding affinity - Mant-ATPYS (and other labeled nucleotides) binding to PAN were analyzed as described previously with slight modifications $^{8}$. Briefly, mant-ATPYS binding to PAN was monitored by increase of fluorescence by protein binding at ex $360 \mathrm{~nm} /$ em $440 \mathrm{~nm}$ on a BioTek synergy mx 96 well plate reader. The reaction was run at room temperature in $50 \mathrm{mM}$ Tris $(\mathrm{pH} 7.5), 5 \%$ 
glycerol and $20 \mathrm{mM} \mathrm{MgCl} 2$ with indicated concentration of PAN and nucleotide $(0.015$ $\mu \mathrm{M})$.

Substrate binding - Substrate binding to PAN was monitored by measuring fluorescence polarization (FP). Each WT and mutants PAN protein $(0.1 \mu \mathrm{M})$ was added to GFP-ssrA $(0.08 \mu \mathrm{M})$ in the presence of $1 \mathrm{mM}$ ADP or $1 \mathrm{mM}$ ATPYS in $50 \mathrm{mM}$ Tris $(\mathrm{pH}$ 7.5), $10 \mathrm{mM} \mathrm{MgCl}$, and $1 \mathrm{mM}$ DTT. After 20 min incubation at $25^{\circ} \mathrm{C}, \mathrm{FP}$ was measured in 96-well plates in Synergy2 BioTek plate reader (ex= $494 \mathrm{~nm} / \mathrm{em}=515 \mathrm{~nm})$. The anisotropy equation used by the biotech softwear is: $r=(\operatorname{lvv}-\mathrm{Ivh}) /(\mathrm{Ivv}+2 \mathrm{Ivh})$.

ATPase Assays - PAN's ATPase activity was measured by a NADH-coupled ATP regeneration assay system in $50 \mathrm{mM}$ Tris- $\mathrm{HCl}(\mathrm{pH} 7.5), 5 \mathrm{mM} \mathrm{MgCl}$, 10 ug BSA, $5 \%$ glycerol, 2U/reaction lactate dehydrogenase (LDH) and 2U/reaction pyruvate kinase (PK) (Sigma), $3 \mathrm{mM}$ phosphoenolpyruvate (PEP), $0.2 \mathrm{mg} / \mathrm{ml} \mathrm{NADH}$, and $2 \mathrm{mM} \mathrm{ATP}$. PAN concentration was $100 \mathrm{nM}$ unless specifically mentioned in the legend. ATPase activity was followed by a loss of NADH absorbance at $340 \mathrm{~nm}$. Kinetic analysis of ATP hydrolysis was done by varying the ATP concentrations (10 $\mu \mathrm{M}$ to $4 \mathrm{mM})$ with a fixed concentration of PAN (100 nM). $V_{\max }, K_{M}$ and Hill coefficient $(h)$ values were obtained by non-linear regression analysis using the Hill equation (Sigma Plot).

Nucleotide binding stoichiometry - Stoichiometry of ATPYS binding to the indicated amount of PAN was determined as described previously with modifications using fluorescent nucleotide (mant-ATPYS) instead of a radiolabeled ATPYS ${ }^{8}$. Briefly, different concentrations of mant-ATPYS were incubated with PAN at room temperature and bound complex was rapidly separated (in approximately $<1 \mathrm{sec}$ ) from the free nucleotide 
by $100 \mu \mathrm{l}$ Sephadex G50 spin column. $2 \mathrm{mM}$ ADP was added to the flow through to compete off the mant-ATPYS from PAN, so that quantitation of the unbound form would be comparable to the independent standard curve, which was used to determine the number of nucleotide bound to the PAN.

Mant-nucleotide dissociation kinetics - The kinetics of mant-ADP and mant-ATPYS dissociation from PAN was obtained by Horiba Fluorolog 3 spectrofluorometer with pneumatically driven SFA-20/SPEX stopped-flow accessories for rapid kinetic acquisitions. $150 \mathrm{nM}$ PAN and $150 \mathrm{nM}$ m-ADP (or mant-ATPyS) were mixed to form a prebound mant-nucleotide complex, which was competed off with excess amount of ADP (2-4 mM) using stopped-flow injection. The dissociation curve of m-nucleotide from PAN was analyzed with Oracle by fitting to either single or double exponential decay models to derive the dissociation half-life $\left(T_{1 / 2}\right)$.

FRET measurements - FRET experiments were done by mixing the $1 \mu \mathrm{M}$ donor MantATP and $1 \mu \mathrm{M}$ acceptor TNP (Trinitrophenyl)-ATP to the $1 \mu \mathrm{M}$ PAN in assay buffer (50 $\mathrm{mM}$ Tris- $\mathrm{HCl}(\mathrm{pH} 7.5), 10 \mathrm{mM} \mathrm{MgCl}, 1 \mathrm{mM} \mathrm{DTT})$ for 20 min and measurements were made by monitoring donor (Mant) fluorescence decrease (exciting at $350 \mathrm{~nm}$ and scanning emission spectra between 370 and $650 \mathrm{~nm}$ ) to observe fluorescence resonance energy transfer on a BioTek 96 well plate reader. This FRET pair has been previously used to determining the distance between two bound nucleotides in a Gprotein dimer ${ }^{37}$. The acceptor (TNP) fluorescence increase due to FRET was not displayed significantly in emission spectra due to the low quantum yield of TNP fluorescence. All measurements were performed in triplicate, were highly repeatable, 
and the FRET efficiency was calculated by the following equation: $E=1-F_{D A} / F_{D}$, where $E$ is the FRET efficiency, $F_{D A}$ is the fluorescence intensity of the donor with the acceptor present, and $\mathrm{F}_{\mathrm{D}}$ is the fluorescence intensity of the donor without the acceptor. In addition, due to the mixing of FRET pairs with equal affinities $E$ had to be corrected by a factor of $0.5\left(E_{\text {corrected }}=E(1 / 0.5)\right)$ as explained in detail previously ${ }^{37}$. In brief, equal mixing of Mant (M) and TNP (T) nucleotides, would be expected to generate 4 different types of complexes: two that FRET (MT and TM), and two that don't (MM and TT). In this way $50 \%$ of the fluorescent intensity observed comes from the FRET condition and $50 \%$ does not, thus requiring this correction factor.

Distance estimates were calculated by using the equation, $E_{\text {corrected }}=1 /\{1+$ $\left.\left(r / R_{0}\right)^{6}\right\}$, where $E_{\text {corrected }}$ is the FRET efficiency calculated above, $r$ is the actual distance, and $R_{0}$ is the Förster critical distance for the FRET pair. For the Mant and TNP pair, $R_{0}$ is known to be $40 \AA^{37}$. The $\mathrm{k}^{2}$ limits were determined by determining the steady-state (0.17-mant, and 0.25-TNP) and fundamental (0.33 for Mant and TNP) anisotropies of the bound donor and acceptor on a Horiba Fluorolog 3 as described ${ }^{53}$. The calculated $\mathrm{K}_{\min }^{2}$ and $\mathrm{k}_{\max }^{2}$ values are given in Table 1. 
Supplemental Material 
a

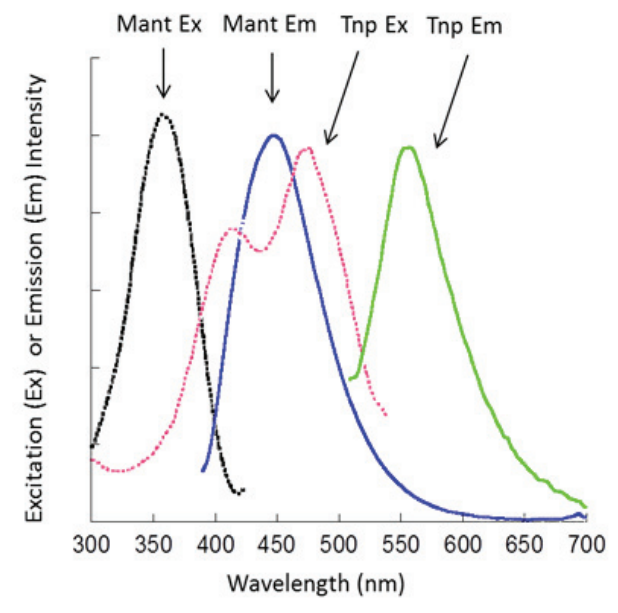

b

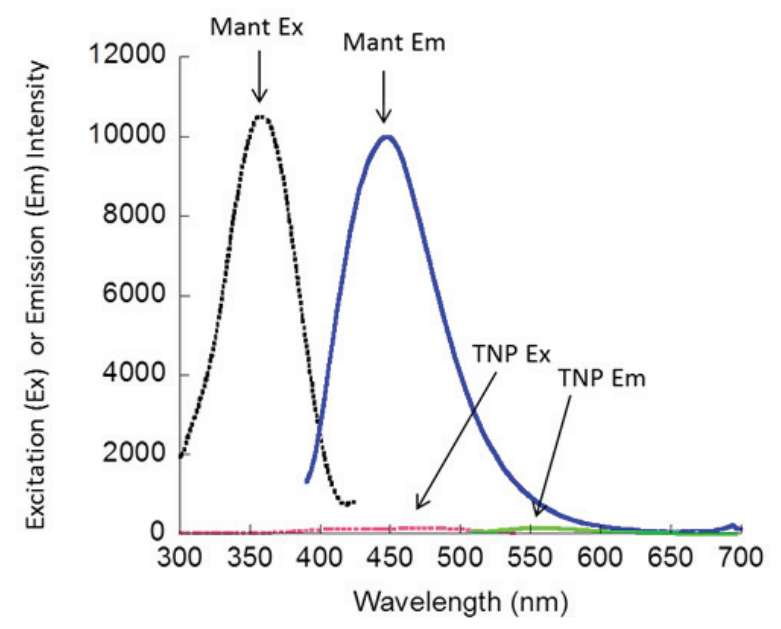

Figure S2.1. Spectra of FRET pair. Normalized (A) and raw (B) absorption and fluorescence spectra of donor (m-ATP) and acceptor (t-ATP) pair used in FRET experiments (Figure 1). 


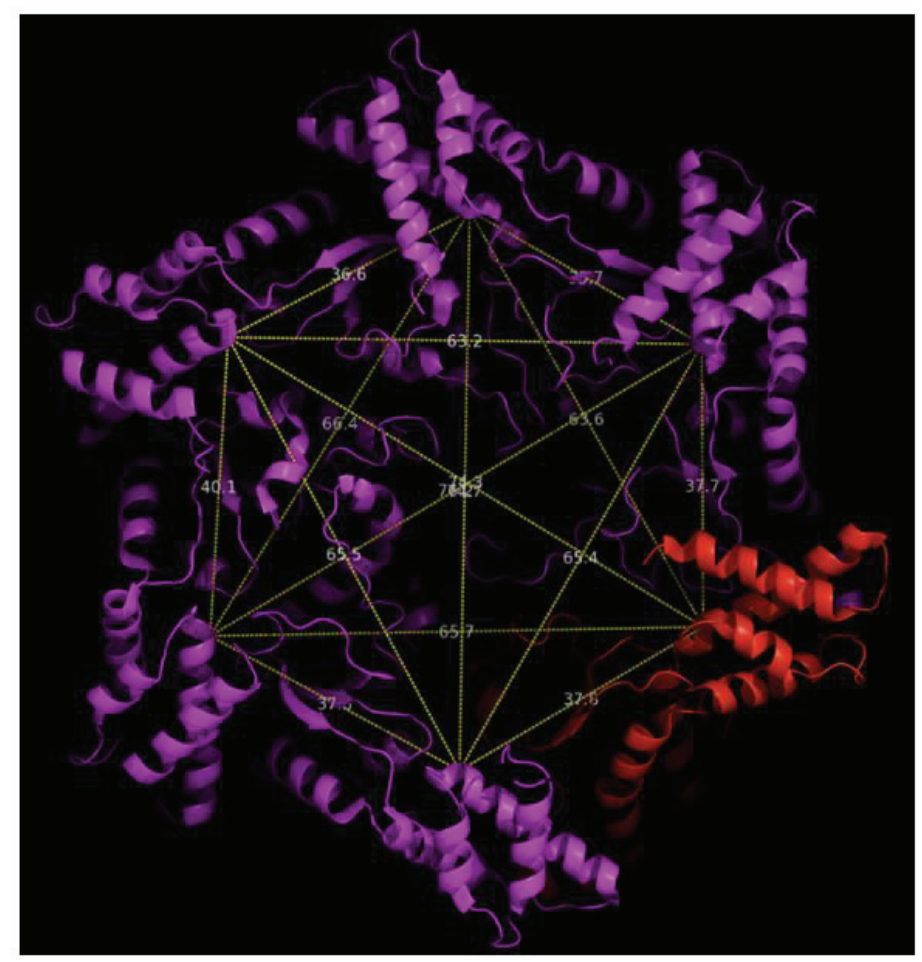

b

\begin{tabular}{|cc|cc|cc|}
\hline Rpt Pair & Ortho & Rpt Pair & Meta & Rpt Pair & Para \\
\hline $2-6$ & 35.7 & $1-6$ & 63.6 & $1-3$ & 74.3 \\
$1-2$ & 37.7 & $2-3$ & 63.2 & $2-4$ & 76.2 \\
$5-1$ & 37.8 & $6-4$ & 66.4 & $6-5$ & 74.7 \\
$4-5$ & 37.8 & $3-5$ & 65.5 & & \\
$3-4$ & 40.1 & $4-1$ & 65.7 & & \\
$6-3$ & 36.6 & $5-2$ & 65.4 & & \\
Average & 37.6 & \multicolumn{5}{|c}{65.0} & \\
\end{tabular}

Figure S2.2. Structural model of the 26S ATPases and estimated distances between nucleotide binding sites. A) Structure of the 26S ATPase complex (PDB:4CR4). Distances between the alanine residues in putative Sensor 2 motif were determined using Pymol. The sensor 2 alanine residue was chosen for distance measurements because it contacts the ribose ring of the nucleotide which is the moiety that is modified by the mant or TNP fluorophores and thus best approximates the distance between the bound FRET pair. The ATPase ring is shown with Cterminal side up, and the red subunit is Rpt1. B) All distances between nucleotide binding sites in the 26S ATPases (PDB:4CR4). Distance is shown in Angstroms and is an estimate since the original source data for the map was determined by cryoEM to a resolution of $\sim 8 \mathrm{nM}$. 

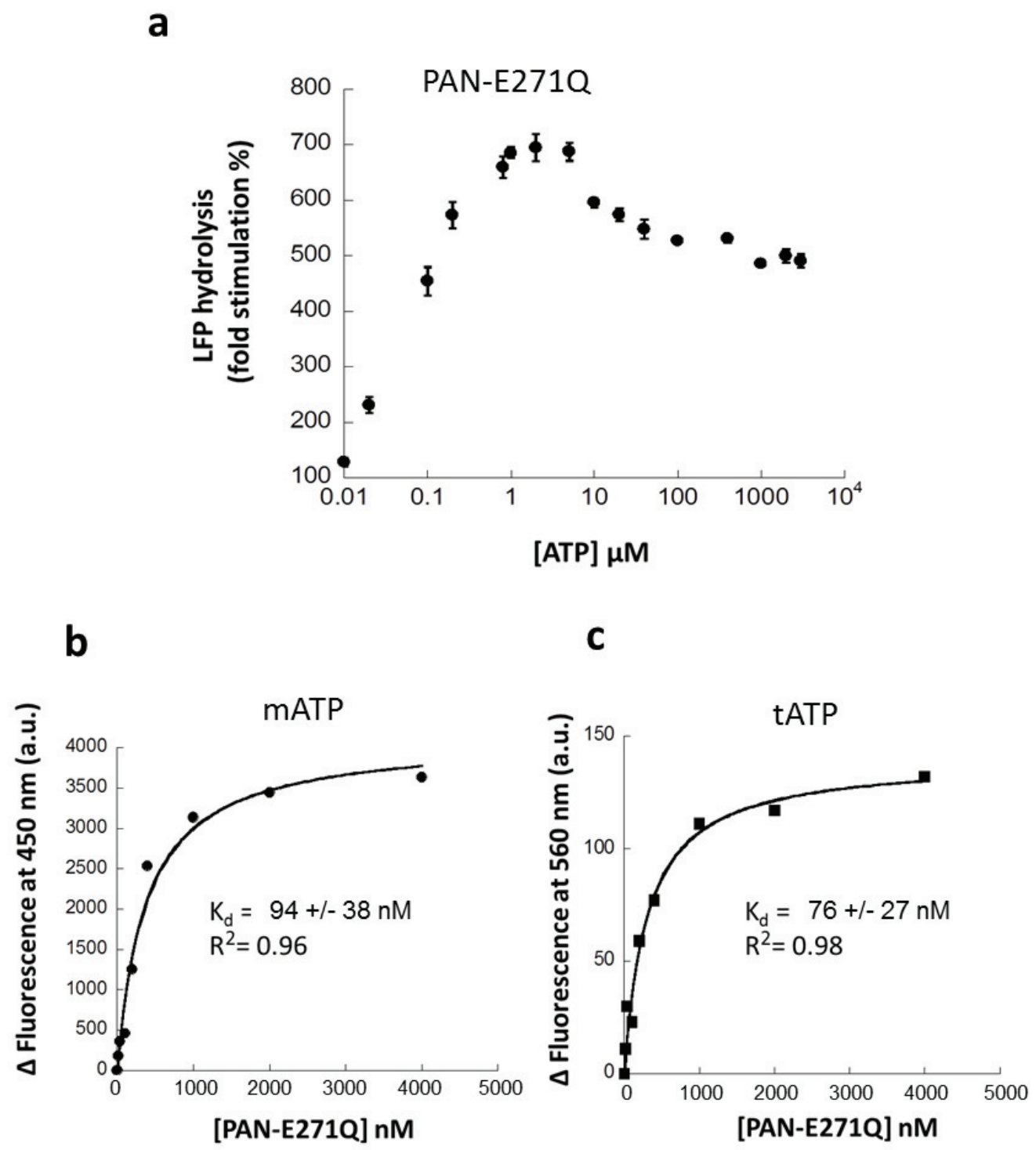

Figure S2.3. ATP binding to PAN-E271Q is biphasic and fluorescent modifications have little effect on ATP affinity. A) Gate opening in the $20 \mathrm{~S}$ proteasome $(20 \mathrm{nM})$ by PAN Walker B mutant (PAN-E271Q) $(80 \mathrm{nM})$ was monitored with the LFP hydrolysis in the presence of a different concentration of ATP. This biphasic curve for $20 \mathrm{~S}$ gate opening was also observed for ATP $\gamma \mathrm{S}$ binding to PAN and correlates with the 2-bound (optimal) and 4-bound (suboptimal) states of PAN (Smith et. al. 2011). The data are the means of three independent measurements \pm S.D. B) Equilibrium ATP binding affinity was determined by monitoring the change in fluorescence intensity of mant-ATP $(0.05 \mu \mathrm{M})$ in the presence of increasing amounts of PAN-E271Q. The 1:1 binding equation (hyperbola) was fit to the raw data points to obtain the shown Kd value. C) Equilibrium ATP binding affinity was also determined by monitoring the change in fluorescence intensity of TNP-ATP $(0.05 \mu \mathrm{M})$ by PANE271Q binding to obtain $\mathrm{Kd}$ value. 
a

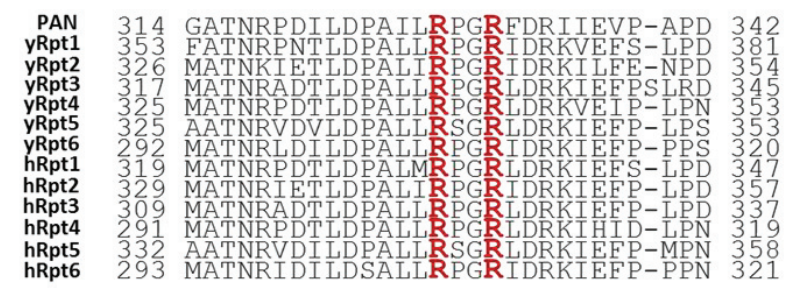

b
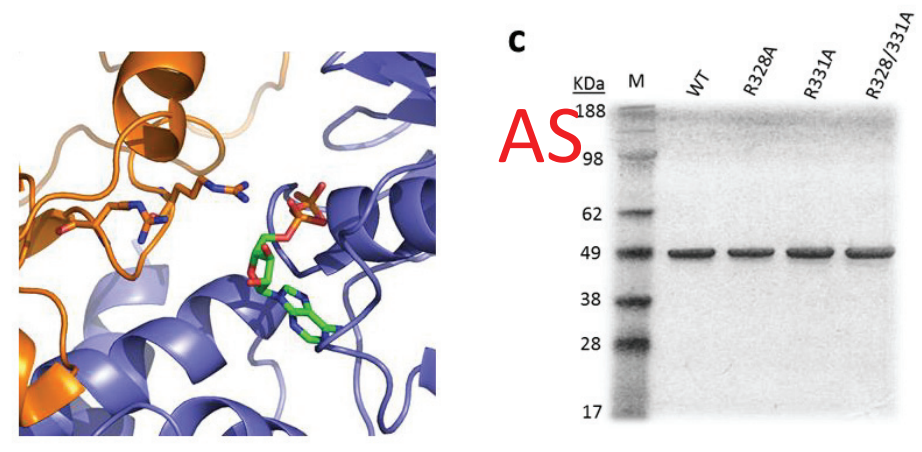

d

AS

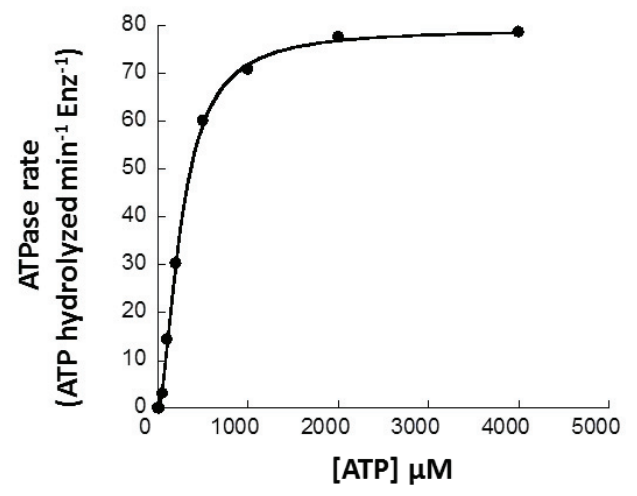

\begin{tabular}{|c|c|c|c|}
\hline $\begin{array}{c}\text { Kinetic } \\
\text { constants }\end{array}$ & $\begin{array}{c}\mathrm{V}_{\max } \\
\left(\mathrm{min}^{-1}\right)\end{array}$ & $\begin{array}{c}\mathrm{K}_{\mathrm{M}} \\
(\mu \mathrm{M})\end{array}$ & $\mathrm{h}$ \\
\hline WT PAN & $79.4 \pm 0.9$ & $261 \pm 8$ & $1.70 \pm 0.07$ \\
\hline
\end{tabular}

Supplementary Figure 2.4. Conserved arginine residues in the proteasomal ATPases and generation of arginine mutants in PAN. A) Alignment of the Secondary Region of Homology (SRH) in the proteasomal ATPases from $M$. jannaschii (PAN), S. cerevisiae (yRpt1-6), and H. sapiens (hRpt1-6), and the conservation of putative arginine finger residues (red and bold). B) Structure of the Rpt2-1 interface (Rpt2-orange and Rpt1-blue) showing the two highlighted conserved arginines (orange rods; PDB:4BGR). To add a nucleotide to the ATP binding site the crystal structure of PAN with nucleotide was fit to RPT1 (green rods). C) Purified PAN (WT) and indicated PAN arginine mutants were resolved by SDS-PAGE and stained with Coomassie. The molecular weight marker (M) is shown. D) The rate of ATP hydrolysis was determined at increasing concentrations of ATP for the WT PAN. The Hill equation was used to fit the data and derive the kinetic constants. Representative data is presented from three independent experiments. 


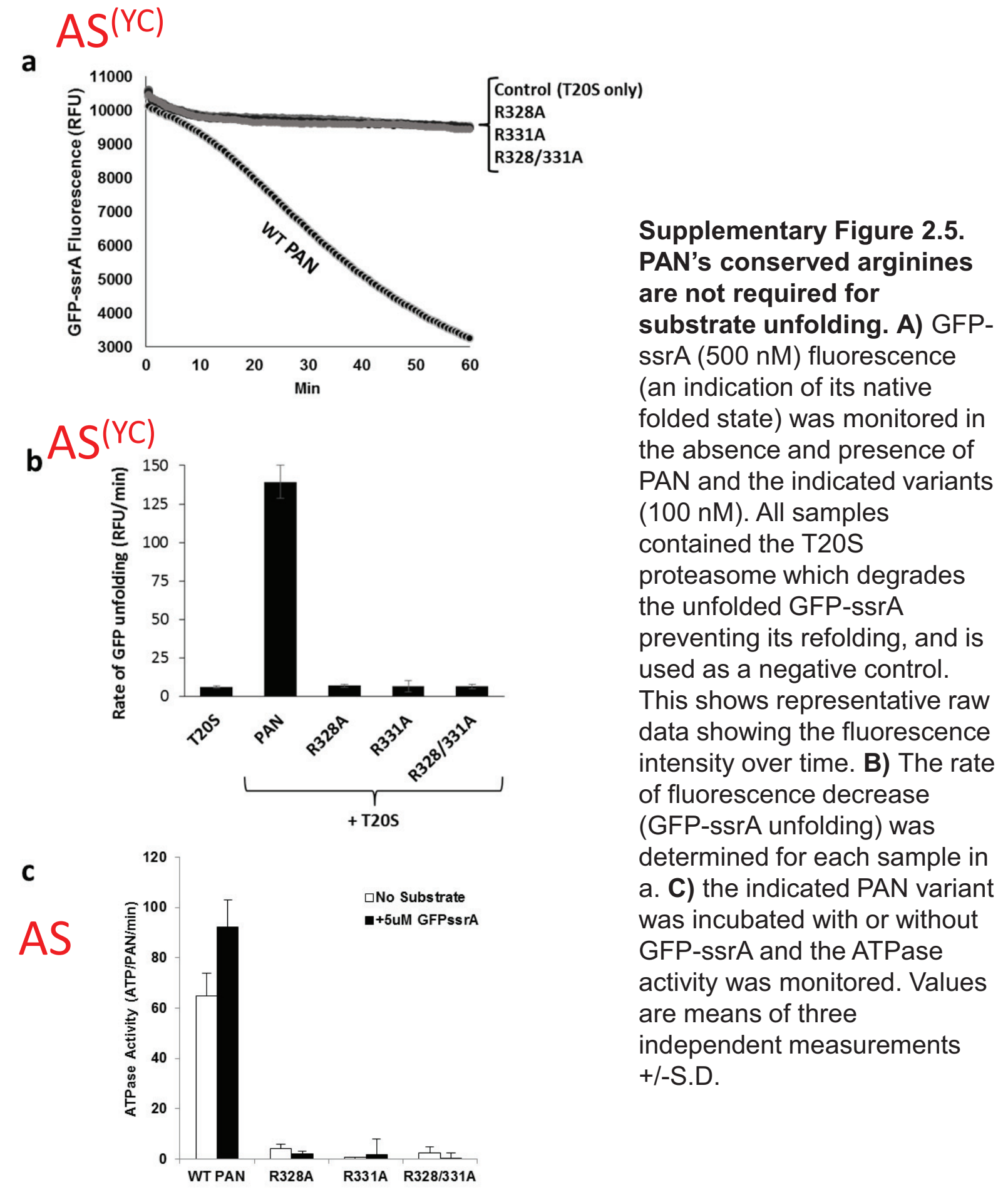




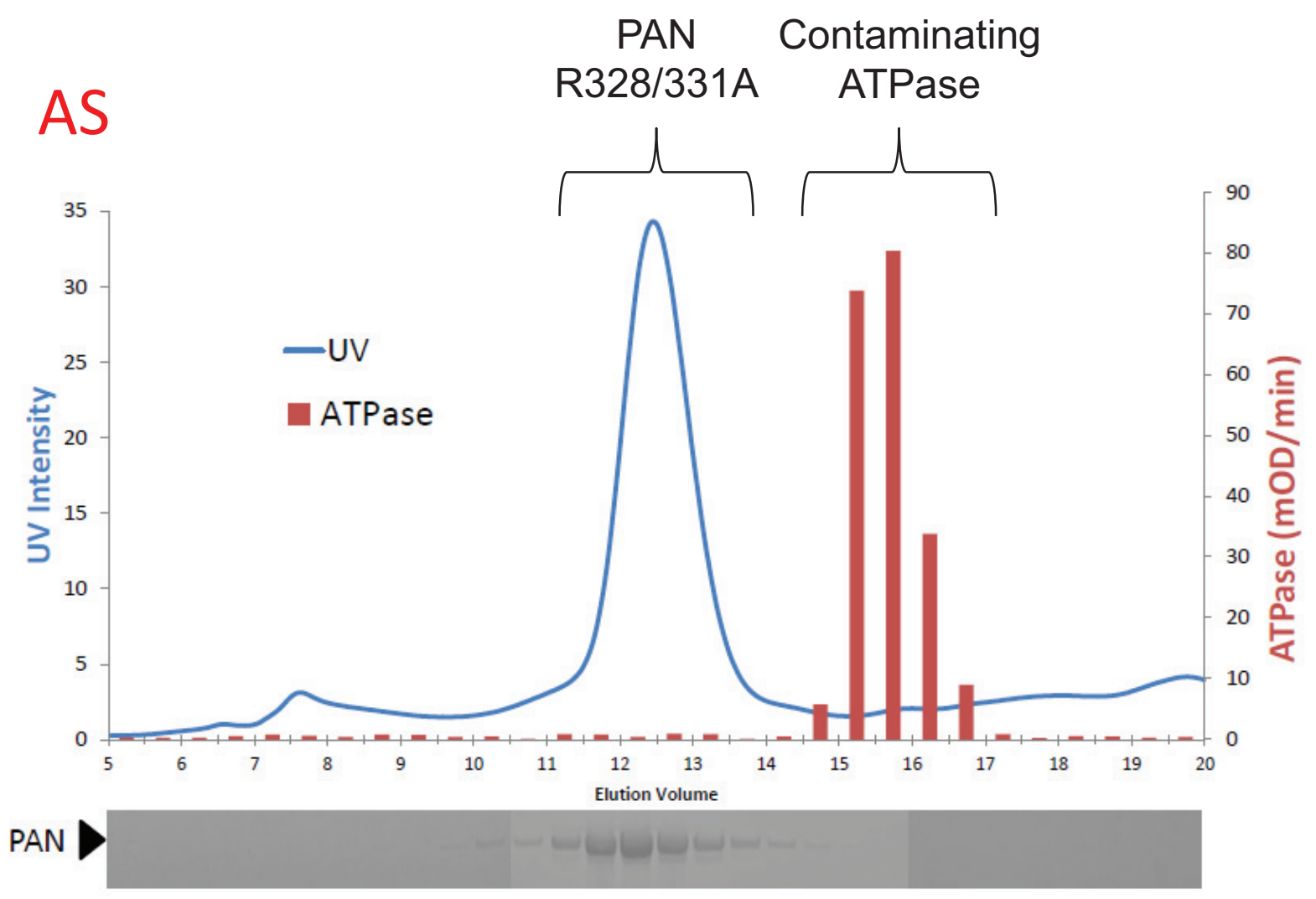

Supplementary Figure 2.6. Careful purification of PAN arginine mutants to eliminate a highly active contaminating ATPase. Figure illustrating that it is imperative to carefully purify PAN arinine finger mutants. Upon size exlusion purification and analysis via SDS-PAGE, one main peak is observed that corresponds to the PAN R328/331A arginine finger double mutant (which does not have ATPase activity). Note that a highly active ATPase (which is not detectable via SDS-PAGE) is observed at later fractions on the size exclusion column. 


\section{References}

1. Tomko, R.J. \& Hochstrasser, M. Molecular architecture and assembly of the eukaryotic proteasome. Annual review of biochemistry 82, 415-445 (2013).

2. Finley, D. Recognition and processing of ubiquitin-protein conjugates by the proteasome. Annu Rev Biochem 78, 477-513 (2009).

3. Bhattacharyya, S., Yu, H., Mim, C. \& Matouschek, A. Regulated protein turnover: snapshots of the proteasome in action. Nature reviews. Molecular cell biology 15, 122-133 (2014).

4. Bar-Nun, S. \& Glickman, M.H. Proteasomal AAA-ATPases: Structure and function. Biochimica Et Biophysica Acta-Molecular Cell Research 1823, 67-82 (2012).

5. Sledz, P. et al. Structure of the $26 \mathrm{~S}$ proteasome with ATP-gammaS bound provides insights into the mechanism of nucleotide-dependent substrate translocation. Proc Natl Acad Sci U S A 110, 7264-9 (2013).

6. Unverdorben, P. et al. Deep classification of a large cryo-EM dataset defines the conformational landscape of the $26 \mathrm{~S}$ proteasome. Proceedings of the National Academy of Sciences of the United States of America 111, 5544-5549 (2014).

7. Matyskiela, M.E., Lander, G.C. \& Martin, A. Conformational switching of the $26 \mathrm{~S}$ proteasome enables substrate degradation. Nat Struct Mol Biol 20, 781-8 (2013).

8. Smith, D.M., Fraga, H., Reis, C., Kafri, G. \& Goldberg, A.L. ATP binds to proteasomal ATPases in pairs with distinct functional effects, implying an ordered reaction cycle. Cell 144, 526-38 (2011).

9. Peth, A., Uchiki, T. \& Goldberg, A.L. ATP-dependent steps in the binding of ubiquitin conjugates to the $26 \mathrm{~S}$ proteasome that commit to degradation. Mol Cell 40, 671-81 (2010).

10. Smith, D.M. et al. ATP binding to PAN or the 26S ATPases causes association with the $20 \mathrm{~S}$ proteasome, gate opening, and translocation of unfolded proteins. Mol Cell 20, 687-98 (2005).

11. Liu, C.W. et al. ATP binding and ATP hydrolysis play distinct roles in the function of 26S proteasome. Mol Cell 24, 39-50 (2006).

12. Prakash, S., Tian, L., Ratliff, K.S., Lehotzky, R.E. \& Matouschek, A. An unstructured initiation site is required for efficient proteasome-mediated degradation. Nat Struct Mol Biol 11, 830-7 (2004). 
13. Zhang, F. et al. Mechanism of substrate unfolding and translocation by the regulatory particle of the proteasome from Methanocaldococcus jannaschii. $\mathrm{Mol}$ Cell 34, 485-96 (2009).

14. Erales, J., Hoyt, M.A., Troll, F. \& Coffino, P. Functional asymmetries of proteasome translocase pore. J Biol Chem 287, 18535-43 (2012).

15. Kim, Y.C., Li, X., Thompson, D. \& DeMartino, G.N. ATP binding by proteasomal ATPases regulates cellular assembly and substrate-induced functions of the $26 \mathrm{~S}$ proteasome. J Biol Chem 288, 3334-45 (2013).

16. Lee, C., Schwartz, M.P., Prakash, S., Iwakura, M. \& Matouschek, A. ATPdependent proteases degrade their substrates by processively unraveling them from the degradation signal. Mol Cell 7, 627-37 (2001).

17. Smith, D.M. et al. Docking of the proteasomal ATPases' carboxyl termini in the $20 \mathrm{~S}$ proteasome's alpha ring opens the gate for substrate entry. Mol Cell 27, 731-44 (2007).

18. Rabl, J. et al. Mechanism of gate opening in the $20 \mathrm{~S}$ proteasome by the proteasomal ATPases. Mol Cell 30, 360-8 (2008).

19. Gillette, T.G., Kumar, B., Thompson, D., Slaughter, C.A. \& DeMartino, G.N. Differential roles of the $\mathrm{COOH}$ termini of AAA subunits of PA700 (19 S regulator) in asymmetric assembly and activation of the $26 \mathrm{~S}$ proteasome. J Biol Chem 283, 31813-22 (2008).

20. Kim, Y.C. \& Demartino, G.N. C termini of proteasomal ATPases play nonequivalent roles in cellular assembly of Mammalian $26 \mathrm{~S}$ proteasome. $\mathrm{J}$ Biol Chem 286, 26652-66 (2011).

21. Lander, G.C. et al. Complete subunit architecture of the proteasome regulatory particle. Nature 482, 186-91 (2012).

22. Lasker, K. et al. Molecular architecture of the $26 \mathrm{~S}$ proteasome holocomplex determined by an integrative approach. Proc Natl Acad Sci U S A 109, 1380-7 (2012).

23. Tian, G. et al. An asymmetric interface between the regulatory and core particles of the proteasome. Nat Struct Mol Biol 18, 1259-67 (2011).

24. Rubin, D.M., Glickman, M.H., Larsen, C.N., Dhruvakumar, S. \& Finley, D. Active site mutants in the six regulatory particle ATPases reveal multiple roles for ATP in the proteasome. EMBO J 17, 4909-19 (1998). 
25. Singleton, M.R., Sawaya, M.R., Ellenberger, T. \& Wigley, D.B. Crystal structure of T7 gene 4 ring helicase indicates a mechanism for sequential hydrolysis of nucleotides. Cell 101, 589-600 (2000).

26. Lyubimov, A.Y., Strycharska, M. \& Berger, J.M. The nuts and bolts of ringtranslocase structure and mechanism. Current opinion in structural biology 21, 240-248 (2011).

27. Hersch, G.L., Burton, R.E., Bolon, D.N., Baker, T.A. \& Sauer, R.T. Asymmetric interactions of ATP with the AAA+ CIpX6 unfoldase: allosteric control of a protein machine. Cell 121, 1017-27 (2005).

28. Stinson, B.M. et al. Nucleotide binding and conformational switching in the hexameric ring of a AAA+ machine. Cell 153, 628-639 (2013).

29. Martin, A., Baker, T.A. \& Sauer, R.T. Rebuilt AAA + motors reveal operating principles for ATP-fuelled machines. Nature 437, 1115-20 (2005).

30. Wendler, P., Ciniawsky, S., Kock, M. \& Kube, S. Structure and function of the AAA+ nucleotide binding pocket. Biochim Biophys Acta 1823, 2-14 (2012).

31. Hanson, P.I. \& Whiteheart, S.W. AAA+ proteins: have engine, will work. Nat Rev Mol Cell Biol 6, 519-29 (2005).

32. Erzberger, J.P. \& Berger, J.M. Evolutionary relationships and structural mechanisms of AAA+ proteins. Annu Rev Biophys Biomol Struct 35, 93-114 (2006).

33. Ogura, T., Whiteheart, S.W. \& Wilkinson, A.J. Conserved arginine residues implicated in ATP hydrolysis, nucleotide-sensing, and inter-subunit interactions in AAA and AAA+ ATPases. J Struct Biol 146, 106-12 (2004).

34. McCullough, J. \& Sundquist, W.I. Putting a finger in the ring. Nature structural \& molecular biology 21, 1025-1027 (2014).

35. Glynn, S.E., Martin, A., Nager, A.R., Baker, T.A. \& Sauer, R.T. Structures of asymmetric ClpX hexamers reveal nucleotide-dependent motions in a AAA+ protein-unfolding machine. Cell 139, 744-56 (2009).

36. Joshi, S.A., Hersch, G.L., Baker, T.A. \& Sauer, R.T. Communication between ClpX and ClpP during substrate processing and degradation. Nat Struct Mol Biol 11, 404-11 (2004).

37. Güldenhaupt, J. et al. N-Ras forms dimers at POPC membranes. Biophysical journal 103, 1585-1593 (2012). 
38. Zhang, F. et al. Structural insights into the regulatory particle of the proteasome from Methanocaldococcus jannaschii. Mol Cell 34, 473-84 (2009).

39. Benaroudj, N., Zwickl, P., Seemuller, E., Baumeister, W. \& Goldberg, A.L. ATP hydrolysis by the proteasome regulatory complex PAN serves multiple functions in protein degradation. Mol Cell 11, 69-78 (2003).

40. Martin, A., Baker, T.A. \& Sauer, R.T. Pore loops of the AAA+ ClpX machine grip substrates to drive translocation and unfolding. Nat Struct Mol Biol 15, 1147-51 (2008).

41. Beckwith, R., Estrin, E., Worden, E.J. \& Martin, A. Reconstitution of the $26 \mathrm{~S}$ proteasome reveals functional asymmetries in its AAA+ unfoldase. Nature structural \& molecular biology 20, 1164-1172 (2013).

42. Peth, A., Nathan, J.A. \& Goldberg, A.L. The ATP costs and time required to degrade ubiquitinated proteins by the $26 \mathrm{~S}$ proteasome. The Journal of biological chemistry 288, 29215-29222 (2013).

43. Stinson, B.M., Baytshtok, V., Schmitz, K.R., Baker, T.A. \& Sauer, R.T. Subunit asymmetry and roles of conformational switching in the hexameric AAA+ ring of ClpX. Nature structural \& molecular biology 22, 411-416 (2015).

44. Sen, M. et al. The ClpXP protease unfolds substrates using a constant rate of pulling but different gears. Cell 155, 636-646 (2013).

45. Glynn, S.E., Nager, A.R., Baker, T.A. \& Sauer, R.T. Dynamic and static components power unfolding in topologically closed rings of a AAA+ proteolytic machine. Nat Struct Mol Biol 19, 616-22 (2012).

46. Zeymer, C., Fischer, S. \& Reinstein, J. trans-Acting arginine residues in the AAA+ chaperone ClpB allosterically regulate the activity through inter- and intradomain communication. The Journal of biological chemistry 289, 32965-32976 (2014).

47. Chen, B. et al. Engagement of arginine finger to ATP triggers large conformational changes in NtrC1 AAA+ ATPase for remodeling bacterial RNA polymerase. Structure (London, England : 1993) 18, 1420-1430 (2010).

48. Beck, F. et al. Near-atomic resolution structural model of the yeast $26 \mathrm{~S}$ proteasome. Proc Natl Acad Sci U S A (2012).

49. Peskin, C.S., Odell, G.M. \& Oster, G.F. Cellular motions and thermal fluctuations: the Brownian ratchet. Biophys J 65, 316-24 (1993).

50. Augustin, S. et al. An intersubunit signaling network coordinates ATP hydrolysis by m-AAA proteases. Mol Cell 35, 574-85 (2009). 
51. Cordova, J.C. et al. Stochastic but highly coordinated protein unfolding and translocation by the ClpXP proteolytic machine. Cell 158, 647-658 (2014).

52. Besche, H.C., Haas, W., Gygi, S.P. \& Goldberg, A.L. Isolation of mammalian $26 \mathrm{~S}$ proteasomes and p97/VCP complexes using the ubiquitin-like domain from HHR23B reveals novel proteasome-associated proteins. Biochemistry 48, 2538-49 (2009).

53. Lakowicz, J. Principles of Fluorescence Spectroscopy; 3rd ed.; Springer, $\mathbf{C h}$. 10, (2006). 
Chapter 3: The Proteasomal ATPases use a slow but highly processive strategy to unfold proteins 


\section{Preface}

In the previous chapter, we discovered that proteasomal ATPases seem to exhibit an ordered binding and hydrolysis mechanism. At the time it seemed as though this result did not agree with a substantial portion of the literature concerning the distantly related ATP-dependent protease from bacteria, ClpX. To shed light on this controversial topic, we performed experiments to compare and contrast the kinetics and mechanochemical coupling of PAN (archaeal proteasomal ATPase) and the 19S (eukaryotic proteasomal ATPase). We also included some review of the literature and background in regards to the current state concerning the kinetics of protein unfolding by other ATP-dependent proteases (e.g. ClpX).

The kinetics for ClpX had been worked out over the course of several studies over the previous few years, and we referenced these studies in relation to ours as appropriate. These studies showed that ClpX stalls when it meets a folded domain (e.g. GFP). Here we asked if the proteasomal ATPases from Archaea and Eukaryotes exhibit similar stalling characteristics when they meet a folded domain. Interestingly, we found that neither of the proteasomal ATPases stalled at the folded domain of GFP, in stark contrast to the ClpX mechanism. We further discuss how differences in the homologous domains, structures, and ATP-hydrolysis cycles between ClpX and the proteasomal ATPases may allow them to use different kinetic strategy for protein unfolding, and we speculated how these strategies may have evolved differently based on the individual needs of their substrate pools. This study has had quite an impact on the field, since up until this point, we did not believe that the field appreciated the differences between 
proteasomal ATPases and ClpX —as each time we proposed a sequential model for PAN and the 19S, we were often cited to be incorrect on the basis of studies on ClpX. This study was the first to suggest that although we propose a sequential mechanism of hydrolysis for the proteasome, this does not contradict the ClpX field, since ClpX and the proteasomal ATPases are different motors that evolved for different functions.

The work presented in this chapter resulted in a $1^{\text {st }}$ author publication for me in Frontiers in Molecular Biosciences. I designed, performed, and analyzed all experiments in this chapter.

Snoberger A, Anderson RH, Smith DM. (2017) "The Proteasomal ATPases use a slow but highly processive strategy to unfold proteins", Frontiers in Molecular Biosciences. 4,4:18. doi:10.3389/fmolb.2017.00018

This chapter has been reprinted here under Creative Commons Attribution 4.0 International License. 


\section{Abstract}

All domains of life have ATP-dependent compartmentalized proteases that sequester their peptidase sites on their interior. ATPase complexes will often associate with these compartmentalized proteases in order to unfold \& inject substrates into the protease for degradation. Significant effort has been put into understanding how ATP hydrolysis is used to apply force to proteins and cause them to unfold. The unfolding kinetics of the bacterial ATPase, ClpX, have been shown to resemble a fast motor that traps unfolded intermediates as a strategy to unfold proteins. In the present study, we sought to determine if the proteasomal ATPases from eukaryotes and archaea exhibit similar unfolding kinetics. We found that the proteasomal ATPases appear to use a different kinetic strategy for protein unfolding, behaving as a slower but more processive and efficient translocation motor, particularly when encountering a folded domain. We expect that these dissimilarities are due to differences in the ATP binding/exchange cycle, the presence of a trans-arginine finger, or the presence of a threading ring (i.e. the OB domain), which may be used as a rigid platform to pull folded domains against. We speculate that these differences may have evolved due to the differing client pools these machines are expected to encounter. 


\section{Introduction}

Virtually every cellular process relies on properly regulated protein degradation. Bacteria, archaea, and eukaryotes all have systems for targeted protein degradation (e.g. the ClpP protease in bacteria and the $20 \mathrm{~S}$ proteasome in archaea and eukaryotes). Both CIpP and the 20S proteasome are capable of degrading unfolded proteins, but since their peptidase sites are sequestered on their hollow interior with only small pores through which substrates can enter, these proteases are not able to degrade folded proteins by themselves because they are too bulky to enter these narrow translocation pores. In order to stimulate degradation of folded proteins, regulatory ATPase complexes associate with the proteolytic complex and use the chemical energy from ATP hydrolysis to unfold and inject the folded proteins into the proteases' central chamber for degradation. While much is understood about this process, we do not have a detailed molecular understanding of how these different ATP-dependent machines engage with and forcibly translocate substrates for selective protein degradation (Alexopoulos et al., 2012; Bar-Nun and Glickman, 2012; Finley, 2009; Mack and Shorter, 2016; Smith et al., 2006; Tomko and Hochstrasser, 2013).

To date some of the better characterized regulatory complexes for the $20 \mathrm{~S}$ proteasome are the heterohexameric 195 regulatory particle in eukaryotes (which forms the 19S-20S, or "26S" complex) and the homohexameric 19S homolog in archaea, PAN (Proteasome Activating Nucleotidase). One of the most extensively studied ClpP regulators is ClpX. In general, the 19S, PAN, and ClpX utilize ATP to: 1) bind and open the gate of their respective protease (Alexopoulos et al., 2013; Grimaud et al., 1998; Liu 
et al., 2006; Smith et al., 2005), 2) recognize proper substrates (Kim et al., 2015; Peth et al., 2010; Smith et al., 2011; Thibault et al., 2006), and 3) unfold and inject them into their protease's degradation chamber (Erales et al., 2012; Ortega et al., 2000; Prakash et al., 2004; Singh et al., 2000; Zhang et al., 2009). All 3 of these regulators are members of the AAA+ superfamily (ㅅTPases associated with diverse cellular activities), but only PAN and the 19S ATPases belong to the same AAA sub-clade, which also contain the SRH region (Lupas and Martin, 2002). Due to the complexities of generating ubiquitinated globular substrates that could be degraded by the purified $26 S$ proteasome, far more functional studies have been done on PAN and CIpX, which only require the presence of a small unfolded region (i.e. ssrA) to trigger substrate degradation (Benaroudj et al., 2003; Hoskins et al., 2002). Although they serve similar functions, ClpX and the proteasomal ATPases may not exhibit similar mechanochemical translocation mechanisms, which would not be unexpected since they each belong to different sub-clades of the AAA+ family. Recent functional studies suggest that they may also have different ATP-hydrolysis characteristics. For example, evidence suggests that ClpX hydrolyzes ATP in a semi-stochastic fashion (Sauer and Baker, 2011), whereas the proteasomal ATPases appear to use an ordered, sequential cycle with a specific "ortho" binding pattern (binding to neighboring subunits) which is subject to expected equilibrium binding considerations (Kim et al., 2015; Smith et al., 2011). Additionally, function-critical allostery between subunits is mediated by the proteasomal ATPase's trans-arginine fingers (Kim et al., 2015), which is lacking in ClpX (Kim and Kim, 2003). These differences in the structure and hydrolysis patterns of ClpX and the 
proteasomal ATPases suggest they may use distinct mechanical strategies to unfold proteins.

Prior studies have shown that when ClpX is translocating on a protein and encounters a stably folded domain (e.g. GFP) it will often stop and even slip backward before taking another run at the folded domain. It's thought that this can occur over and over until spontaneous unfolding occurs after which ClpX quickly translocates onto the unfolded domain, trapping it, and preventing its refolding (Aubin-Tam et al., 2011; losefson et al., 2015b; Maillard et al., 2011; Nager et al., 2011; Rodriguez-Aliaga et al., 2016). ClpX may also perturb the folded domain prior to trapping. This likely continues until the whole domain is unfolded (Fig. 3.1A). In this proposed model ClpX seems to function at high velocity, whereby quick trapping of unfolded intermediates (rather than brute force unfolding) is the primary strategy used to unfold the domain. Alternatively, one can think of this as a motor with high velocity, but with low processivity when it encounters an obstacle to translocation that causes slipping. Interestingly, the ATP hydrolysis rate of ClpX is $\sim 100-500$ ATPs per minute in the absence of substrate (Aubin-Tam et al., 2011; Baytshtok et al., 2015; losefson et al., 2015a; Maillard et al., 2011; Martin et al., 2005; Nager et al., 2011; Rodriguez-Aliaga et al., 2016), which is considerably faster than the $\sim 30-60$ ATPs per minute of the proteasomal ATPases (Hoffman and Rechsteiner, 1996; Kim et al., 2015; Kraut et al., 2012). Consistent with this high velocity, low processivity mechanism, ClpX has been shown to exhibit a nonlinear relationship with regard to its ATPase rate and substrate unfolding rate, especially in more tightly folded substrates (Nager et al., 2011). This is expected since at 

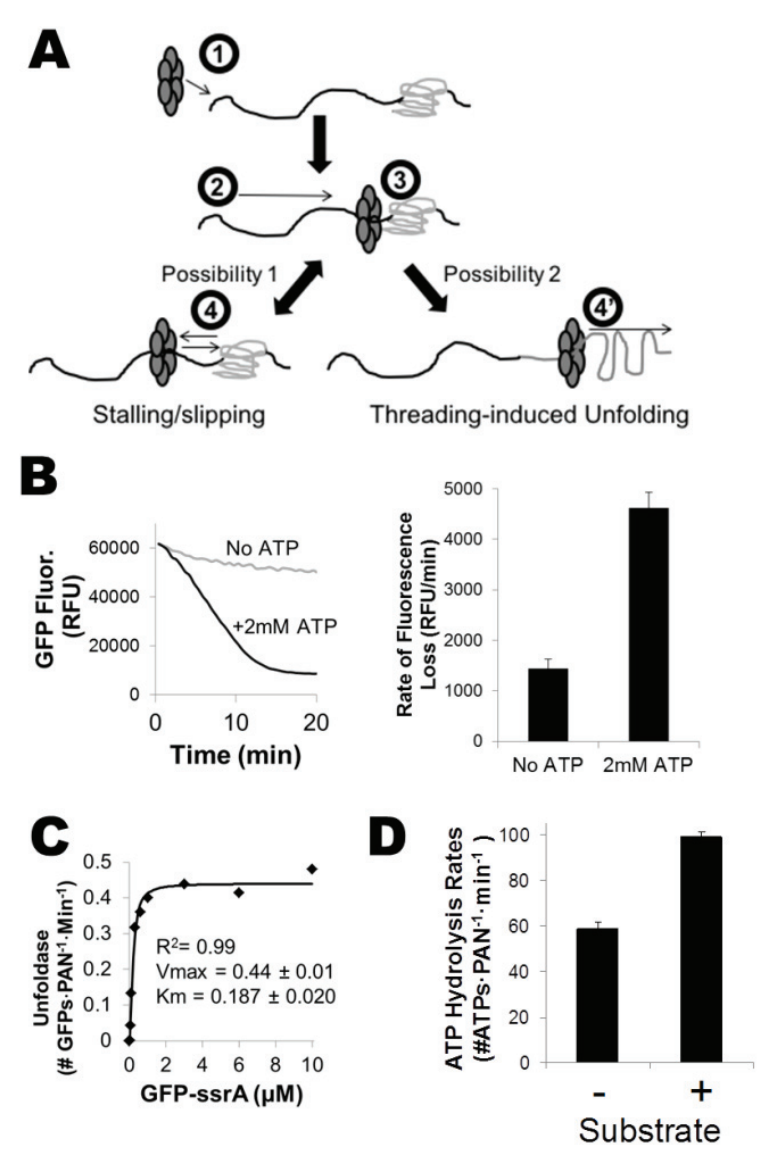

Figure 3.1: Hexameric ATP-dependent proteases utilize energy from ATP hydrolysis to unfold substrates. A) Hexameric ATP-dependent proteases (e.g. ClpX or the proteasomal ATPases) 1) recognize their protein substrates and utilize energy from ATP hydrolysis to thread the protein through their central pore to 2) translocate along the unfolded region of the protein until they 3 ) reach a folded domain. 4) Less processive ATP-dependent proteases have a tendency to slip once they reach a more tightly folded domain, and if the ATP hydrolysis rates slow below a critical threshold they will stall and even slip backward before taking another run at the folded domain. 4') More processive ATPases (or less processive ATPases after multiple runs at the folded domain) are able to drive through these more tightly folded domains to cause threading-induced unfolding of this protein domain, followed by further translocation along the protein. B) The ATP-dependent GFPssrA substrate unfolding rate was measured in reaction buffer (see methods) including 200nM GFPssrA, 50nM PAN, 400nM T20S, and with and without saturating ATP (2mM). Unfolding of GFPssrA was assessed by quantifying the steady-state rate of fluorescence loss (ex/em: 485/510). C) GFPssrA unfolding kinetics were determined the same as in (A), but with varying amounts of GFPssrA (from 0-10 $\mu \mathrm{M}$ ). D) Summary of ATPase rates with and without substrate for the proteasomal ATPases. ATPase rates for PAN were determined at $2 \mathrm{mM}$ ATP using a kinetic NADH-coupled assay, with and without saturating GFPssrA $(2 \mu \mathrm{M})$. Error bars are standard deviations from 3 independent experiments $(n=3)$. 
saturating ATP concentrations $\mathrm{ClpX}$ is able to translocate at maximal rates and trap unfolded intermediates, but when the ATPase rate is slowed (by using lower ATP concentrations or by competing with non-hydrolyzable ATPYS) the net translocation rate is also slowed when the unfolded intermediates refold before ClpX can trap them. Thus, at lower ATP hydrolysis rates ATP hydrolysis becomes non-productive and ClpX continually slips on the substrate without productive translocation (Fig. 3.1A). This model for ClpX translocation kinetics has also been supported with single-molecule force experiments (Aubin-Tam et al., 2011; losefson et al., 2015b; Maillard et al., 2011; Rodriguez-Aliaga et al., 2016).

In the present study we ask if the proteasomal ATPases have translocation and unfolding kinetics that are consistent with this model of ClpX, or if its structural and mechanochemical differences allow it to take a different strategy for substrate unfolding. We show that, unlike CIpX, the 195 and PAN proteasomal ATPases resemble a lower velocity, but highly processive motor that is slower than ClpX but does not appear to stall when it approaches the stably folded domain of GFP, but rather it drives through it without slipping. These kinetics are consistent with the hand over hand sequential mechanism of ATP hydrolysis that has been proposed for the proteasomal ATPases (Kim et al., 2015; Smith et al., 2011). These data therefore suggest that proteasomal ATPases, while slower, are more processive and efficient than ClpX and use a different kinetic strategy for unfolding substrates. 


\section{Results}

In order to test unfolding ability of PAN, we used the model substrate of GFP with an unstructured ssrA tag fused to its N-terminus (GFPssrA). GFP's fluorescence is dependent on its tertiary structure; therefore, the rate of unfolding can be monitored by following its decrease in fluorescence in real time. As expected, PAN unfolded GFPssrA in an ATP-dependent manner (Fig. 3.1B). The slow loss of GFP fluorescence in the "no ATP" control is attributed to the slow bleaching of GFP with time, which is expected. To determine the catalytic affinity $(\mathrm{Km})$ for GFP we performed a GFPssrA dose response at saturating $[A T P](2 \mathrm{mM})$. The unfolding rate was determined by calculating the maximum linear rate of the change in GFP fluorescence with time. The Vmax of GFPssrA unfolding was $0.44 \pm 0.01$ GFPs $\cdot \mathrm{PAN}^{-1} \cdot$ minute $^{-1}$, which indicates that PAN takes $\sim 2$ minutes to unfold a single GFP. This unfolding rate for the proteasomal ATPases is consistent with prior observations (Benaroudj et al., 2003). In addition, the $\mathrm{Km}$ was found to be $0.187 \mu \mathrm{M}$ (Fig. 3.1C). Next, we determined the ATP hydrolysis rate in PAN using a real-time NADH-coupled assay and found the rate of ATP hydrolysis to be 58.5 \pm 3.5 ATPs $\cdot$ PAN $^{-1} \cdot$ minute $^{-1}$ in the absence of substrate and was activated $\sim 1.7$ fold to $97.0 \pm 2.9$ ATPs $\cdot$ PAN $^{-1} \cdot$ minute $^{-1}$ upon addition of saturating GFPssrA $(2 \mu \mathrm{M})$, which is also consistent with previous reports (Kim et al., 2015) (Fig. 3.1D). The ATP hydrolysis rate we found for PAN is fairly similar to previous reports in the mammalian $26 \mathrm{~S}$ proteasome, which place the ATPase rates between $\sim 30-50$ ATPs per minute in the absence of substrate (Hoffman and Rechsteiner, 1996; Kraut et al., 2012), with a 1.5-2 fold activation upon addition of substrate (Peth et al., 2013). We compared this ATP hydrolysis rate to previously reported ATP hydrolysis rates for the psueudohexameric 
ClpX. Reported ATPase rates for the ClpX pseudohexamer tend to vary quite a bit ( 100-500 ATPs per minute) (Aubin-Tam et al., 2011; Baytshtok et al., 2015; losefson et al., 2015a; Maillard et al., 2011; Martin et al., 2005; Nager et al., 2011; Rodriguez-Aliaga et al., 2016), but all of these rates are considerably faster than the reported basal rates for the proteasomal ATPases. Addition of substrate to ClpX typically increases its ATP hydrolysis rate, although the degree to which ClpX is activated depends upon the substrate analyzed (Baytshtok et al., 2015; losefson et al., 2015a; Kenniston et al., 2003).

A longstanding question in the proteasomal ATPase field is how chemical energy from ATP is converted into mechanical work on substrates, and the efficiency of such mechanochemical coupling is informative to mechanism. In ClpX, it was found that at higher ATPase rates, ClpX has quite efficient mechanochemical coupling; however, at lower ATPase rates coupling is less efficient (i.e. at lower ATPase rates, ATP hydrolysis often does not lead to unfolding). This less efficient mechanochemical coupling can be observed by decreasing the rate of ATP hydrolysis by either reducing total [ATP] or competing with nonhydrolyzable nucleotide. In order to test the mechanochemical coupling efficiency of PAN, we simultaneously measured, in real time, the unfolding rate of GFPssrA and PAN's ATPase activity (via absorbance of NADH in a coupled ATPase assay-Methods). $0.2 \mu \mathrm{M}$ GFPssrA $(\sim \mathrm{Km})$ was incubated with PAN at various concentrations of ATP to determine the ATPase (Fig. 3.2A) and unfoldase rates (Fig. 3.2B). To our surprise, Km values of PAN's ATPase and GFPssrA unfolding 

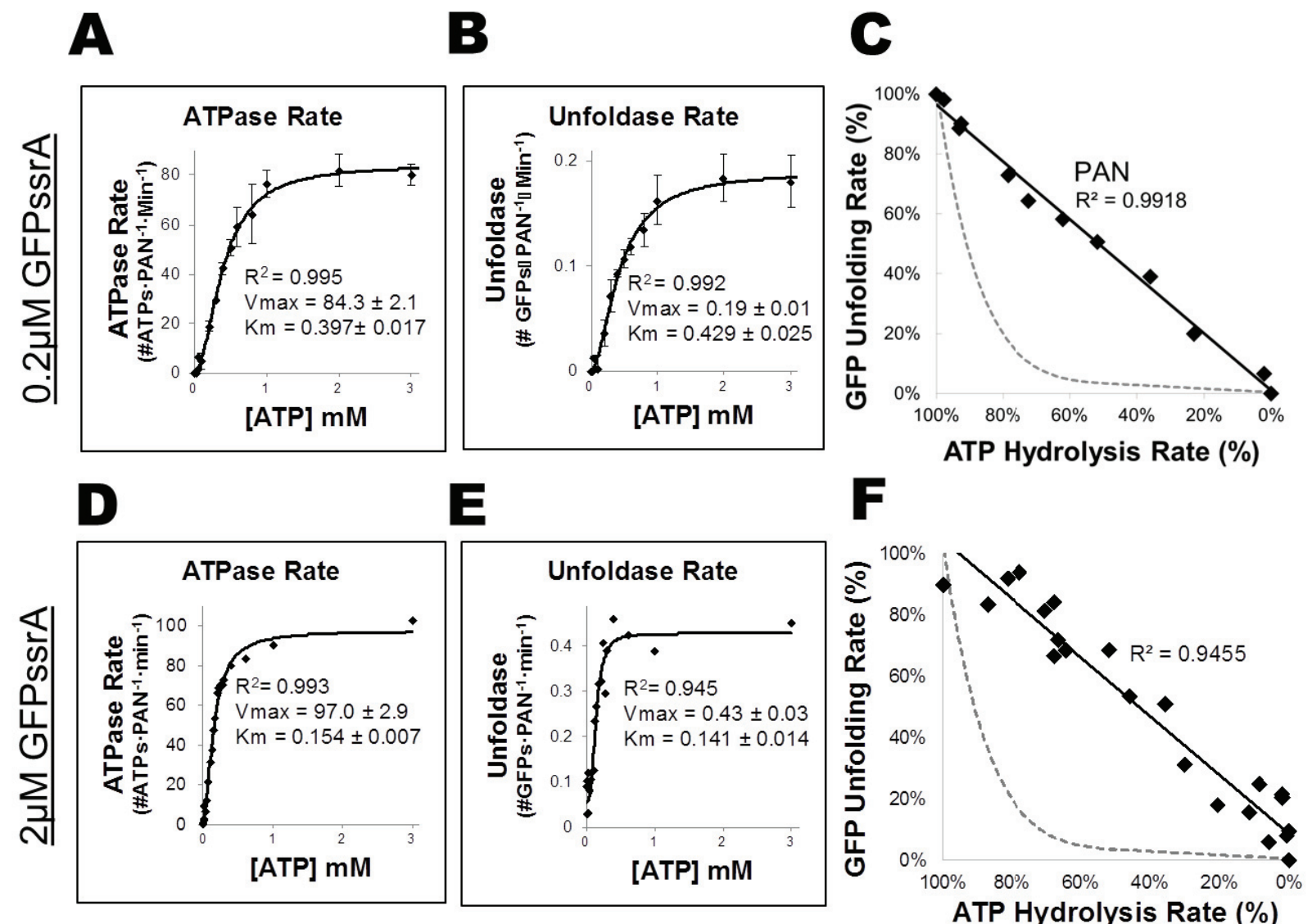

Figure 3.2: PAN does not stall when it encounters the unfolded domain of GFP. A-B) To determine mechanochemical coupling efficiency at $\sim \mathrm{Km}$ levels of GFPssrA, ATP hydrolysis and GFPssrA unfolding $(2 \mu \mathrm{M})$ were assessed concurrently, in the same well, using an NADH-coupled ATPase assay combined with GFPssrA unfolding (see methods). Rate of ATP hydrolysis was measured by loss of NADH absorbance at $340 \mathrm{~nm}(\mathrm{~A})$, while at the same time GFPssrA unfolding rate was measured by loss of fluorescence at ex/em: 485/510 (B). C) Efficiency of mechanochemical coupling of ATP hydrolysis to GFPssrA was determined by plotting relative $\%$ ATPase and unfoldase onto a 2 dimensional plot and fitting with a line $\left(R^{2}=0.9918\right)$. The dotted gray line is a hypothetical example of an ATPase that stalls (e.g. ClpX), where stalling is defined as $<5 \%$ of the maximal degradation rate when the ATPase rate is $50 \%$ of maximal (Nager et al. 2011). D-F). Same as (A-C), but at saturating GFPssrA substrate concentration $(2 \mu \mathrm{M})$. Error bars are standard deviations from 3 independent experiments $(n=3)$ 
matched quite well with one another, with the $\mathrm{Km}$ of ATPase activity being $0.397 \pm$ $0.017 \mu \mathrm{M}$ and the $\mathrm{Km}$ for GFPssrA unfolding being $0.429 \pm 0.025 \mu \mathrm{M}$. This suggested a tight coupling between unfolding and ATPase rates at least around $1 / 2 \mathrm{Vmax}$. We then plotted the GFP unfolding and ATP hydrolysis rates against each other on a single 2dimensional plot (Fig. 3.2C). Surprisingly, the data was very linear and fit a linear curve with an $R^{2}$ of 0.9918 . Therefore, PAN exhibits a $1: 1$ mechanochemical coupling of ATPase and unfoldase activities. In contrast, prior experiments with ATPases that stall (e.g. ClpX) have shown that its ATPase to GFPssrA unfoldase plot is highly non-linear (e.g. when the ATPase rate is $\sim 50 \%$, the unfolding rate drops to $<5 \%$ ). In Figures 3.2C\&F, we show a dotted gray line as an example of what the ATPase vs. unfoldase plot would look like in a stalling ATPase (e.g. ClpX). This nonlinear ATPase to GFPssrA unfoldase relationship has been attributed to increased substrate "stalling" and "slipping" upon reaching a globular domain (i.e. GFP's beta-barrel), which results in nonproductive ATP hydrolysis (Aubin-Tam et al., 2011; losefson et al., 2015b; Maillard et al., 2011; Nager et al., 2011; Rodriguez-Aliaga et al., 2016). Since we found that PAN's ATPase activity is directly proportional (1:1) to GFPssrA unfolding, this data indicates that PAN essentially does not slip when it reaches the folded domain of the GFP beta-barrel. We repeated the experiment using saturating levels of GFPssrA $(2 \mu \mathrm{M})$ and found that the Km for ATPase activity and GFP unfolding were nearly identical to one another (Fig. 3.2D-E). Consistent with Figure 3.1C, the Vmax for unfolding was 2fold higher at saturating [GFPssrA] $\left(0.43 \pm 0.03\right.$ GFPs $\cdot$ PAN $^{-1} \cdot$ minute $\left.^{-1}\right)$ (Fig. 3.2E) compared to at the Vmax at $\sim \mathrm{Km}$ concentrations of GFPssrA $\left(0.19 \pm 0.01 \mathrm{GFPs}^{\mathrm{PAN}}{ }^{-}\right.$ ${ }^{1}$. minute $^{-1}$ ) (Fig. 3.2B). This is expected since the unfolding rate at $\mathrm{Km}$ concentrations of 
GFPssrA should be $1 / 2$ of the Vmax. Consistent with prior observations, we observed here that saturating levels of GFPssrA stimulated the Vmax for ATPase activity by $\sim 1.7$ fold when compared to no substrate ATPase experiments (Fig. 3.1D), and a $\sim 1.2$ fold increase when compared to the 200nM GFPssrA experiments (Fig. 3.2D). Interestingly, we found that in addition to increasing the Vmax, saturating levels of GFPssrA also lowered the $\mathrm{Km}$ for ATP hydrolysis and substrate unfolding approximately 2-3 fold (compare $\mathrm{Km}$ values in Fig. 3.2A-B to $\mathrm{Km}$ values in Fig. 3.2D-E). This may suggest an underlying mechanism for substrate stimulated ATPase activity, which is well established in the literature. In addition, the similar $\mathrm{Km}$ between ATPase and unfoldase activities at saturating substrate levels is consistent with the linear fit $\left(R^{2}=0.9455\right)$ that we observe when plotting ATP hydrolysis against GFP unfolding (Fig. 3.2F), similar to Figure 3.2C. Thus, even when all PAN complexes are bound to a GFPssrA the rate of ATP hydrolysis is tightly coupled to GFP unfolding. In other words, hydrolysis of ATP by PAN almost always results in a successful translocation event, even when it meets a globular domain.

The eukaryotic 19S ATPases are homologous to PAN, however, the 195 forms a heterohexameric ring and has many additional associated non-ATPase subunits while PAN forms a homohexameric ring and has no known non-ATPase subunits. Therefore, it was unclear whether the 1:1 mechanochemical coupling of ATPase rate to substrate unfolding that we observed in PAN would be a general property of proteasomal ATPases, or whether it would only apply to the archaeal proteasomal ATPases. Therefore, we sought to determine whether the eukaryotic 26S (i.e. 19S-20S complex) 
also had a similar linear relationship between its ATPase and unfoldase activity. The Matouschek group generously provided us with a novel $26 \mathrm{~S}$ substrate, Ub ${ }^{4}$ (lin)-GFP ${ }^{35}$ $\mathrm{His}^{6}$, suitable for use with in vitro $26 \mathrm{~S}$ unfolding assays. Such a substrate is very useful for mechanistic studies since it allows for the analysis of ubiquitin- and ATP-dependent degradation using the purified $26 \mathrm{~S}$ proteasome. For the $26 \mathrm{~S}$ proteasome to remain functional it requires the persistent presence of ATP, so we could not assess coupling of ATPase and substrate unfolding using the ATP dose response as was done in Figure 3.2 for PAN because low ATP concentrations would induce disassembly of the $26 \mathrm{~S}$ proteasome (Thompson et al., 2009). Instead, we slowed ATPase rate by competing ATP with the largely non-hydrolyzable ATP analogue, ATPYS (which by itself stabilizes the $26 \mathrm{~S}$ complex as does ATP). We first performed this ATPYS competition experiment in PAN and found that as the ATPYS:ATP ratio increased, GFPssrA unfolding rate decreased in a 1:1 linear relationship with the ATPYS:ATP ratio $\left(R^{2}=0.989\right)$ (Fig. 3.3A). This is consistent with and further supports our observations with the ATP dose response method in Figure $3.2 \mathbf{C} \& \mathbf{F}$, and it demonstrates that the ATPYS:ATP ratio method mimics a linear decrease in ATP hydrolysis activity in PAN similar to the ATP dose response. We next performed a similar ATPYS competition experiment using the $\mathrm{Ub}^{4}$ (lin)-GFP ${ }^{35}$ substrate and the eukaryotic $26 \mathrm{~S}$ proteasome and were surprised to find that the 26S had similar 1:1 unfolding kinetics to that observed in PAN (Fig. 3.3B) with a strong linear fit $\left(R^{2}=0.982\right)$. These ATPYS competition experiments demonstrate that ATP hydrolysis and unfolding are also tightly coupled in ubiquitin-dependent protein degradation by the eukaryotic $26 \mathrm{~S}$ proteasome. In addition, this indicates that the tight 


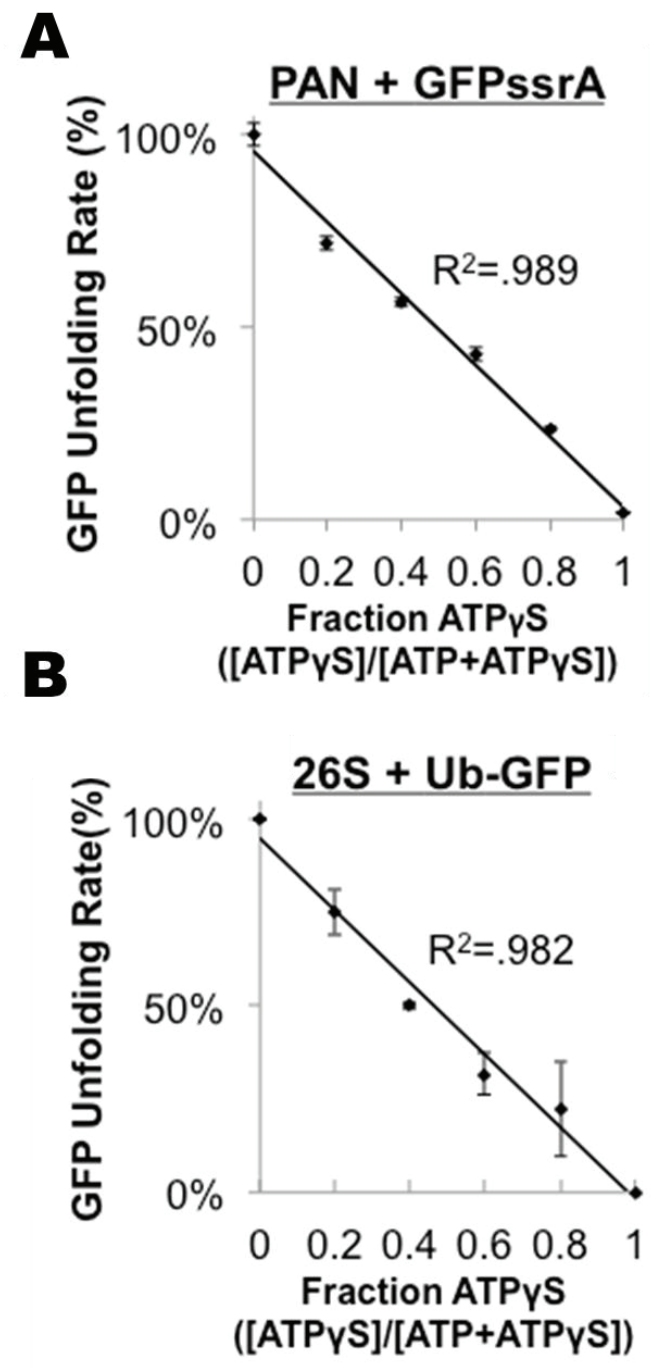

Figure 3.3: The eukaryotic $26 \mathrm{~S}$ does not stall when it encounters the folded domain of GFP. A) PAN's ATPase rate was slowed by competing with increasing ratios of ATPYS:ATP $(2 \mathrm{mM}$ total nucleotide), and GFPssrA $(0.2 \mu \mathrm{M})$ unfolding rate was assessed as in Figure 1A. Data fit a line with an $R^{2}=0.989$. B) $25 n M$ of purified rabbit $26 \mathrm{~S}$ was incubated with $100 \mathrm{nM} \mathrm{Ub}^{4}$ (lin)-GFP 35 and was analyzed as in (A). Data fit a line with an $\mathrm{R}^{2}$ value of 0.982 . "Stalling" is defined in Figure 2. Error bars are standard deviations from 3 independent experiments $(n=3)$ 
mechanochemical coupling between ATP hydrolysis and unfolding ability is shared between PAN and the $26 \mathrm{~S}$ and thus it is expected to be a general property of the proteasomal ATPases despite their structural differences. 


\section{Discussion}

Previous studies reveal that the bacterial ClpX pseudohexamer resembles a higher velocity motor. It also has a correspondingly quick steady-state translocation rate: for example $\sim 7$ amino acids per second on the "non-stalling" substrate, cp6 ${ }^{\text {SF GFPssrA }}$ (Nager et al., 2011). However, when ClpX reaches a tightly folded domain "stalling" and "slipping" can occur, whereby it loses its grip on the substrate and the substrate is often released, resulting in unproductive ATP hydrolysis (Aubin-Tam et al., 2011; losefson et al., 2015b; Maillard et al., 2011; Nager et al., 2011; Rodriguez-Aliaga et al., 2016). (Fig. 3.4A-B). In contrast, the proteasomal ATPases hydrolyze ATP considerably more slowly than does ClpX and we estimate that proteasomal ATPases translocate on nonstalling substrates at an average rate of $\sim 1.0-1.9$ amino acids per second, or about 2-7 times more slowly than ClpX. Interestingly, despite these differences in translocation velocity both PAN and ClpX show a similar cost for non-stalling translocation at a mean of $\sim 1.1-1.2$ amino acids translocated per ATP that is hydrolyzed (Fig. 3.4A). Despite this similarity, here we find for the proteasomal ATPases that even at low ATPase rates ATP hydrolysis is tightly coupled with translocation, which is the force that drives unfolding. This is consistent with a lack of substrate "slipping", and indicates that proteasomal ATPases are more efficient and processive than ClpX particularly when they reach a folded domain. Therefore, the proteasomal ATPases operate at a lower velocity, but also have higher processivity since they do not slip or lose grip on the substrate (Fig. 3.4A-B). This suggests that ClpX and PAN utilize different kinetic strategies to unfold proteins: ClpX uses a fast translocation strategy to 
A $\quad$ ClpX ATPase vs. Proteasomal ATPases

\begin{tabular}{|c|c|c|c|}
\hline & \multirow[b]{2}{*}{$\begin{array}{c}\text { ClpX } \\
\text { (Bacteria) }\end{array}$} & \multicolumn{2}{|c|}{ Proteasomal ATPases } \\
\hline & & $\begin{array}{c}19 S \\
\text { (Eukaryotes) }\end{array}$ & $\begin{array}{c}\text { PAN } \\
\text { (Archaea) }\end{array}$ \\
\hline ATP Binding Pattern & $\begin{array}{c}\text { Semi- } \\
\text { stochastic }\end{array}$ & $\begin{array}{l}\text { Neighboring } \\
\text { Subunits }\end{array}$ & $\begin{array}{l}\text { Neighboring } \\
\text { Subunits }\end{array}$ \\
\hline Rigid N-domain? & No & $\begin{array}{c}\text { Yes } \\
\text { OB domain }\end{array}$ & $\begin{array}{c}\text { Yes } \\
\text { OB domain }\end{array}$ \\
\hline Arginine Finger & Cis & Trans & Trans \\
\hline $\begin{array}{c}\text { ATP Hydrolysis Rate } \\
\left.\text { (\#ATPs hydrolyzed.enzyme }{ }^{-1} \cdot \text { minute }^{-1}\right)\end{array}$ & $\sim 100-500$ & $\sim 30-50$ & $\sim 60$ \\
\hline $\begin{array}{l}\text { Steady State translocation rate } \\
\left(\text { amino acids } \cdot \mathrm{sec}^{-1}\right)\end{array}$ & $\sim 7$ & $\sim 1.0$ & $\sim 1.9$ \\
\hline $\begin{array}{c}\text { Translocation Cost }{ }^{\mathrm{c}} \\
\text { (amino acids translocated per ATP hydrolyzed) }\end{array}$ & $\sim 1.2$ & n.d. & $\sim 1.1$ \\
\hline Stalling at folded GFP Domain?d & Yes & No & No \\
\hline Processivity at GFP Domain & Low & High & High \\
\hline
\end{tabular}

B
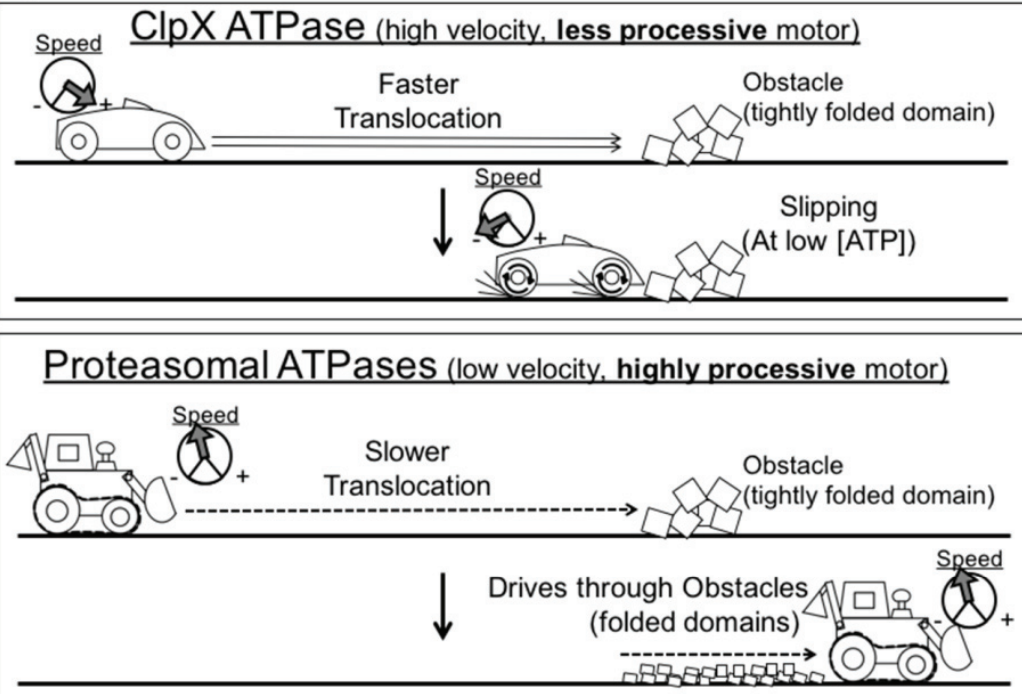

Figure 3.4: Comparison of the unfolding kinetics for the Proteasomal ATPases vs. ClpX. A) Summary of ClpX and the proteasomal ATPases' unfolding kinetics taken from experiments performed in this manuscript as well as by other groups (cited in main text). Footnotes: aATP hydrolysis rate in the absence of substrate. bSteady state translocation rates are taken from mean unfolding rates with nonstalling substrates. ${ }^{~ T}$ Translocation cost is calculated as the rate of steady state translocation on a nonstalling substrate, divided by the ATPase rate of the enzyme on that same substrate. ${ }^{d}$ Stalling is defined as $<5 \%$ of max unfolding rate at $50 \%$ max ATPase activity (Nager et al. 2011) B) Working model: ClpX ATPases resemble a higher velocity, less processive motor that is prone to slipping. ClpX translocates rather quickly along a loosely folded protein domain. However, at low ATP concentrations, ClpX is unable to drive through tightly folded protein domains, and thus undergoes multiple slips and stalls, and can even dissociate from the protein completely. Proteasomal ATPases resemble a lower velocity, more processive motor. The proteasomal ATPases translocate more slowly along a loosely folded protein domain, but even at these lower speeds the proteasomal ATPase is able to drive through more tightly folded domains (i.e. GFP) without significant slipping or stalling. 
trap unfolded intermediates, while the proteasomal ATPases use a slower but more processive and efficient kinetic strategy to drive through unfolded domains with a tight mechanochemical coupling between ATP hydrolysis and translocation events.

What functional characteristics in these ATPases could cause these different kinetic strategies for unfolding proteins? One possibility is the sequential vs. semistochastic mechanisms that have been proposed for the proteasomal ATPases vs. CIpX (Fig. 3.4A). It could be expected that a semi-stochastic ATP-hydrolysis mechanism could lead to states of the ring where all ATPs are hydrolyzed, leaving ClpX in an ADPbound state only. Since ATP binding drives substrate association, this could lead to loss of substrate affinity and slipping, especially when ATP is limiting. In contrast, it has been proposed that the proteasomal ATPases use a sequential single subunit progression mechanism for ATP hydrolysis (Kim et al., 2015). In this model, at least one ATPase subunit is always bound to an ATP, supporting constant affinity for the substrate, which would be expected to prevent slipping. In this model it would thus be expected that most hydrolysis events are coupled to translocation events, which is supported by our data presented here. This tight mechanochemical coupling can be explained by two different models for the proteasomal ATPases: 1) ATP hydrolysis has sufficient power to forcibly unfold GFP with each power stroke, allowing the ATPase to drive through unfolded domains or 2) ATP hydrolysis does not occur in any one subunit until translocation can take place. These two models could represent differences in the "power stroke" versus "Brownian ratchet" mechanisms, and many ATPase motors exhibit a blending of both of these mechanisms, but neither of these have been determined for the proteasomal 
ATPases. However, both models are consistent with the data we have shown here. It's also possible that other structural differences between ClpX and the proteasomal ATPases could play a role in the unfolding kinetics. For example, the proteasomal ATPases have trans-arginine fingers (vs. cis-arginine fingers in ClpX), which constitutes an arginine that allows one subunit to contact the gamma phosphate of the ATP bound to the Walker A/B sites in its neighboring subunit. This arginine is critical for the effects of ATP-binding in the proteasomal ATPases, which include promoting substrate binding, and the association of PAN/19S with the $20 \mathrm{~S}$ core particle and gate-opening. The placement and allosteric role of this trans-arginine is a fundamental difference between the proteasomal ATPases and ClpX. In addition, the role of the trans-arginine finger combined with the single subunit progression model produces a hand-over-hand translocation model that would be expected to exhibit a high "grip" strength mechanism that allows for high substrate binding affinity even at low ATP (Kim et al., 2015). The proteasomal ATPases also contain a rigid ring of $\mathrm{OB}$ domains that substrates are threaded through during translocation. This threading ring generates a rigid platform that folded domains can be pulled against during translocation to cause unfolding. The lack of such a domain in ClpX means that globular domains are pulled into and against the ATPase domains themselves during translocation (especially for the $\Delta \mathrm{N}-\mathrm{ClpX}$ which is used in most of the in vitro experiments that study translocation), which could sterically alter their activity during forceful pulling, and could perhaps cause slipping as well (Fig. 3.4A). 
So why might these 2 distinct mechanisms have evolved for unfolding proteins? In bacteria, ssrA tags are added to the C-terminus of translationally stalled proteins on ribosomes. In fact, approximately 1 in 200 translated proteins are tagged by ssrA, and of these, $>90 \%$ are degraded by $\mathrm{ClpX}(\mathrm{P})$ (Lies and Maurizi, 2008). The vast majority of these translationally stalled proteins will produce truncated proteins, which will typically prevent proper folding, thus destabilizing these proteins. These truncated proteins must also be rapidly degraded in order to prevent aggregation and/or toxicity to the cell. Therefore, a high-velocity unfoldase like ClpX is well suited to quickly handle such proteins, and perhaps ClpX would only rarely be expected to encounter a more tightly folded protein, which could be handled by other ATPases in bacteria such as CIpA. On the other hand, here we have observed that the proteasomal ATPases resemble a lower velocity motor with a more processive and efficient translocation mechanism. Why might this be? The proteasome degrades most proteins in the cell, both unfolded as well as fully folded, functional proteins. Thus, in order for the proteasome to function optimally for this job it must be able to routinely handle more tightly folded domains than ClpX typically encounters. The high processivity, low velocity characteristics that we have observed here for the proteasomal ATPases seem to be optimized for its specific client pool of proteins that demand reliable degradation of folded and functional proteins. Therefore, we propose that the need to unfold and degrade most folded proteins in the cell is the reason that the proteasomal ATPases use a slower but more processive strategy for protein unfolding and degradation. 


\section{Methods}

\section{Materials, plasmids, and protein purification}

PAN, GFPssrA, and T20S were prepared as described (Smith et al., 2005, 2007). The purest available forms of ATP, and ATPyS were purchased from Sigma and stored at $80{ }^{\circ} \mathrm{C}$ until use. Rabbit muscle $26 \mathrm{~S}$ proteasome was purified by the previously described UBL-UIM method (Besche et al., 2009) and were exchanged with reaction buffer by rapid spin column or by dialysis $(4 \mathrm{~h})$ immediately prior to use.

$\mathrm{Ub}^{4}$ (lin)-GFP ${ }^{35}-\mathrm{His}^{6}$ plasmid was a generous gift from Andreas Matouschek and his lab. Plasmids were transfected into $\mathrm{DH} 5 \alpha$ cells, and $1 \mathrm{~L}$ cultures were grown at $37^{\circ}$ at 300RPM shaking, and induced with IPTG at $\mathrm{OD}_{600}=0.8$ for 4 hours. Cell pellets were resuspended in Buffer $A(50 \mathrm{mM}$ Tris $\mathrm{pH} 7.5,5 \%$ glycerol, $300 \mathrm{mM} \mathrm{NaCl}, 20 \mathrm{mM}$ Imidazole) with 1X protease inhibitor cocktail. Cells were lysed via sonication and spun at $20000 \times$ for 30 minutes. Supernatant was loaded onto Nickel-NTA, washed with 10CV Buffer A, and eluted with Buffer B (Buffer A w/ 300mM Imidazole). Fractions containing $\mathrm{Ub}^{4}$ (lin)-GFP ${ }^{35}-\mathrm{His}^{6}$ were pooled based on fluorescence (ex/em: 485/510) and SDS-PAGE. Pooled fractions were concentrated and further purified using sizeexclusion chromatography (GE Superose 12 column). Purest fractions were exchanged into $50 \mathrm{mM}$ Tris $\mathrm{pH} 7.5+5 \%$ glycerol.

\section{ATPase and GFPssrA Unfolding assays}

ATP hydrolysis was measured by reading the loss of NADH absorbance at $340 \mathrm{~nm}$ in an NADH-coupled ATP regenerating system (50mM Tris pH 7.5, 5\% glycerol, $20 \mathrm{mM}$ 
$\mathrm{MgCl} 2,2 \mathrm{U} / \mu \mathrm{l}$ Pyrivate Kinase, $2 \mathrm{U} / \mu \mathrm{l}$ Lactate dehydrogenase, $3 \mathrm{mM}$ phosphoenolpyruvate, and $0.2 \mathrm{mg} / \mathrm{ml} \mathrm{NADH}$, and indicated [ATP]). GFPssrA unfolding was assessed by loss of fluorescence at ex/em: 485/510. For the unfolding experiments, reaction buffer $(50 \mathrm{mM}$ Tris $\mathrm{pH} 7.5,5 \%$ glycerol, $20 \mathrm{mM} \mathrm{MgCl} 2)$ was incubated with 50nM PAN, 400nM T20S, and 0.2nM GFPssrA (or 25nM 26S and 100nM Ub ${ }^{4}$ (lin)-GFP ${ }^{35}-\mathrm{His}^{6}$ for experiments with 26S) and 2mM ATP (or with indicated ATPyS:ATP ratios with $2 \mathrm{mM}$ total nucleotide). GFP fluorescence loss (ex/em: 485/510) was measured every 20 seconds in a Biotek 96 well plate reader to obtain unfolding rates. Error bars represent standard deviations from at least 3 independent experiments $(n \geq 3)$.

ATP hydrolysis and GFPssrA unfolding were assessed concurrently in a Biotek 96 well plate reader by measuring NADH absorbance loss alongside GFPssrA fluorescence loss. The ATP regenerating system buffer (above) was incubated with indicated [ATP] (0-3mM), 50nM PAN, 400nM T20S, and $0.2 \mu \mathrm{M}$ or $2 \mu \mathrm{M}$ GFPssrA. Rates of ATP hydrolysis and GFPssrA unfolding were extrapolated and Vmax and $\mathrm{Km}$ values were obtained by nonlinear regression analysis on Sigmaplot using the Hill equation. Error bars are standard deviations from at least 3 independent experiments $(n \geq 3)$ 


\section{References}

Alexopoulos, J. A., Guarné, A., and Ortega, J. (2012). ClpP: A structurally dynamic protease regulated by AAA+ proteins. J. Struct. Biol. 179, 202-210. doi:10.1016/j.jsb.2012.05.003.

Alexopoulos, J., Ahsan, B., Homchaudhuri, L., Husain, N., Cheng, Y. Q., and Ortega, J. (2013). Structural determinants stabilizing the axial channel of ClpP for substrate translocation. Mol. Microbiol. 90, 167-180. doi:10.1111/mmi.12356.

Aubin-Tam, M.-E., Olivares, A. O., Sauer, R. T., Baker, T. A., and Lang, M. J. (2011). Single-molecule protein unfolding and translocation by an ATP- fueled proteolytic machine. Cell 145, 257-267. doi:doi:10.1016/j.cell.2011.03.036. Single-molecule.

Bar-Nun, S., and Glickman, M. H. (2012). Proteasomal AAA-ATPases: structure and function. Biochim. Biophys. Acta 1823, 67-82. doi:10.1016/j.bbamcr.2011.07.009.

Baytshtok, V., Baker, T. A., and Sauer, R. T. (2015). Assaying the kinetics of protein denaturation catalyzed by AAA + unfolding machines and proteases. doi:10.1073/pnas.1505881112.

Benaroudj, N., Zwickl, P., Seemüller, E., Baumeister, W., and Goldberg, A. L. (2003). ATP hydrolysis by the proteasome regulatory complex PAN serves multiple functions in protein degradation. Mol. Cell 11, 69-78. Available at: http://www.ncbi.nlm.nih.gov/pubmed/12535522.

Besche, H. C., Haas, W., Gygi, S. P., and Goldberg, A. L. (2009). Isolation of mammalian 26S proteasomes and p97/VCP complexes using the ubiquitin-like domain from HHR23B reveals novel proteasome-associated proteins. Biochemistry 48, 2538-2549. doi:10.1021/bi802198q.

Erales, J., Hoyt, M. A., Troll, F., and Coffino, P. (2012). Functional asymmetries of proteasome translocase pore. J. Biol. Chem. 287, 18535-18543. doi:10.1074/jbc.M112.357327.

Finley, D. (2009). Recognition and Processing of Ubiquitin-Protein Conjugates by the Proteasome. Annu. Rev. Biochem. 78, 477-513. doi:10.1146/annurev.biochem.78.081507.101607.Recognition.

Grimaud, R., Kessel, M., Beuron, F., Steven, a. C., and Maurizi, M. R. (1998). Enzymatic and Structural Similarities between theEscherichia coli ATP-dependent Proteases, ClpXP and ClpAP. J. Biol. Chem. 273, 12476-12481. doi:10.1074/jbc.273.20.12476.

Hoffman, L., and Rechsteiner, M. (1996). Nucleotidase activities of the 26 S proteasome and its regulatory complex. J. Biol. Chem. 271, 32538-32545. 
doi:10.1074/jbc.271.51.32538.

Hoskins, J. R., Yanagihara, K., Mizuuchi, K., and Wickner, S. (2002). ClpAP and CIpXP degrade proteins with tags located in the interior of the primary sequence. Proc. Natl. Acad. Sci. U. S. A. 99, 11037-42. doi:10.1073/pnas.172378899.

losefson, O., Nager, A. R., Baker, T. a, and Sauer, R. T. (2015a). Coordinated gripping of substrate by subunits of an AAA+ proteolytic machine. Nat. Chem. Biol. doi:10.1038/nchembio.1732.

Iosefson, O., Olivares, A. O., Baker, A., Sauer, R. T., losefson, O., Olivares, A. O., et al. (2015b). Dissection of Axial-Pore Loop Function during Unfolding and Translocation by a AAA+ Proteolytic Machine. Cell Rep. 12, 1032-1041. doi:10.1016/j.celrep.2015.07.007.

Kenniston, J. A., Baker, T. A., Fernandez, J. M., and Sauer, R. T. (2003). Linkage between ATP consumption and mechanical unfolding during the protein processing reactions of an AAA+ degradation machine. Cell 114, 511-520. doi:10.1016/S00928674(03)00612-3.

Kim, D. Y., and Kim, K. K. (2003). Crystal Structure of ClpX Molecular Chaperone from Helicobacter pylori. J. Biol. Chem. 278, 50664-50670. doi:10.1074/jbc.M305882200.

Kim, Y.-C., Snoberger, A., Schupp, J., and Smith, D. M. (2015). ATP binding to neighbouring subunits and intersubunit allosteric coupling underlie proteasomal ATPase function. Nat. Commun. 6, 8520. doi:10.1038/ncomms9520.

Kraut, D. A., Israeli, E., Schrader, E. K., Patil, A., Nakai, K., Nanavati, D., et al. (2012). Sequence- and species-dependence of proteasomal processivity. ACS Chem. Biol. 7, 1444-1453. doi:10.1021/cb3001155.

Lies, M., and Maurizi, M. R. (2008). Turnover of endogenous SsrA-tagged proteins mediated by ATP-dependent proteases in Escherichia coli. J. Biol. Chem. 283, 22918-22929. doi:10.1074/jbc.M801692200.

Liu, C. W., Li, X., Thompson, D., Wooding, K., Chang, T. ling, Tang, Z., et al. (2006). ATP Binding and ATP Hydrolysis Play Distinct Roles in the Function of $26 \mathrm{~S}$ Proteasome. Mol. Cell 24, 39-50. doi:10.1016/j.molcel.2006.08.025.

Lupas, A. N., and Martin, J. (2002). AAA proteins. Curr. Opin. Struct. Biol. 12, 746-753. doi:10.1016/S0959-440X(02)00388-3.

Mack, K. L., and Shorter, J. (2016). Engineering and Evolution of Molecular Chaperones and Protein Disaggregases with Enhanced Activity. Front. Mol. Biosci. 3, 8. doi:10.3389/fmolb.2016.00008. 
Maillard, R. A., Chistol, G., Sen, M., Righini, M., Tan, J., Kaiser, C. M., et al. (2011). $\operatorname{ClpX}(\mathrm{P})$ generates mechanical force to unfold and translocate its protein substrates. Cell 145, 459-469. doi:10.1016/j.cell.2011.04.010.

Martin, A., Baker, T. a, and Sauer, R. T. (2005). Rebuilt AAA + motors reveal operating principles for ATP-fuelled machines. Nature 437, 1115-20. doi:10.1038/nature04031.

Nager, A. R., Baker, T. A., and Sauer, R. T. (2011). Stepwise unfolding of a beta barrel protein by the AAA+ ClpXP protease. J. Mol. Biol. 413, 4-16. doi:10.1016/j.jmb.2011.07.041.

Ortega, J., Singh, S. K., Ishikawa, T., Maurizi, M. R., and Steven, A. C. (2000). Visualization of substrate binding and translocation by the ATP-dependent protease, ClpXP. Mol. Cell 6, 1515-1521. doi:10.1016/S1097-2765(00)00148-9.

Peth, A., Nathan, J. A., and Goldberg, A. L. (2013). The ATP costs and time required to degrade ubiquitinated proteins by the $26 \mathrm{~s}$ proteasome. J. Biol. Chem. 288, 2921522. doi:10.1074/jbc.M113.482570.

Peth, A., Uchiki, T., and Goldberg, A. L. (2010). ATP-Dependent steps in the binding of ubiquitin conjugates to the $26 \mathrm{~s}$ proteasome that commit to degradation. Mol. Cell 40, 671-681. doi:10.1016/j.molcel.2010.11.002.

Prakash, S., Tian, L., Ratliff, K. S., Lehotzky, R. E., and Matouschek, A. (2004). An unstructured initiation site is required for efficient proteasome-mediated degradation. Nat. Struct. Mol. Biol. 11, 830-7. doi:10.1038/nsmb814.

Rodriguez-Aliaga, P., Ramirez, L., Kim, F., Bustamante, C., and Martin, A. (2016). Substrate-translocating loops regulate mechanochemical coupling and power production in AAA+ protease ClpXP. Nat. Struct. Mol. Biol. 23, 974-981. doi:10.1038/nsmb.3298.

Sauer, R. T., and Baker, T. a (2011). AAA+ proteases: ATP-fueled machines of protein destruction. Annu. Rev. Biochem. 80, 587-612. doi:10.1146/annurev-biochem060408-172623.

Singh, S. K., Grimaud, R., Hoskins, J. R., Wickner, S., and Maurizi, M. R. (2000). Unfolding and internalization of proteins by the ATP-dependent proteases CIpXP and ClpAP. Proc. Natl. Acad. Sci. U. S. A. 97, 8898-903. doi:10.1073/pnas.97.16.8898.

Smith, D. M., Benaroudj, N., and Goldberg, A. (2006). Proteasomes and their associated ATPases: a destructive combination. J. Struct. Biol. 156, 72-83. doi:10.1016/j.jsb.2006.04.012.

Smith, D. M., Chang, S.-C., Park, S., Finley, D., Cheng, Y., and Goldberg, A. L. (2007). 103 
Docking of the proteasomal ATPases' carboxyl termini in the $20 \mathrm{~S}$ proteasome's alpha ring opens the gate for substrate entry. Mol. Cell 27, 731-44. doi:10.1016/j.molcel.2007.06.033.

Smith, D. M., Fraga, H., Reis, C., Kafri, G., and Goldberg, A. L. (2011). ATP binds to proteasomal ATPases in pairs with distinct functional effects, implying an ordered reaction cycle. Cell 144, 526-38. doi:10.1016/j.cell.2011.02.005.

Smith, D. M., Kafri, G., Cheng, Y., Ng, D., Walz, T., and Goldberg, A. L. (2005). ATP binding to PAN or the 26S ATPases causes association with the 20 S proteasome, gate opening, and translocation of unfolded proteins. Mol. Cell 20, 687-98. doi:10.1016/j.molcel.2005.10.019.

Thibault, G., Yudin, J., Wong, P., Tsitrin, V., Sprangers, R., Zhao, R., et al. (2006). Specificity in substrate and cofactor recognition by the N-terminal domain of the chaperone ClpX. Proc. Natl. Acad. Sci. U. S. A. 103, 17724-9. doi:10.1073/pnas.0601505103.

Thompson, D., Hakala, K., and DeMartino, G. N. (2009). Subcomplexes of PA700, the $19 \mathrm{~S}$ regulator of the $26 \mathrm{~S}$ proteasome, reveal relative roles of AAA subunits in $26 \mathrm{~S}$ proteasome assembly and activation and ATPase activity. J. Biol. Chem. 284, 24891-24903. doi:10.1074/jbc.M109.023218.

Tomko, R. J., and Hochstrasser, M. (2013). Molecular Architecture and Assembly of the Eukaryotic Proteasome. Annu. Rev. Biochem. 82, 415-445. doi:10.1146/annurevbiochem-060410-150257.Molecular.

Zhang, F., Wu, Z., Zhang, P., Tian, G., Finley, D., and Shi, Y. (2009). Mechanism of Substrate Unfolding and Translocation by the Regulatory Particle of the Proteasome from Methanocaldococcus jannaschii. Mol. Cell 34, 485-496. doi:10.1016/j.molcel.2009.04.022. 
Chapter 4: Conformational switching in the coiled-coil domains of a proteasomal ATPase regulates substrate processing 


\section{Preface}

Chapters 2-3 in this dissertation clearly show that although PAN is a homohexamer, its subunits appear to adopt non-identical conformations when binding and hydrolyzing ATP, which allows the subunits to processively unfold and degrade substrates. We wondered what structural components allowed the AAA+ ATPase portion of PAN to exhibit this conformational heterogeneity. We initially hypothesized that perhaps PAN's subunits were held in these distinct conformations by their $\mathrm{N}$-terminal coiled-coil (CC) domains (similar to how CC domains of other proteins can regulate various structural elements). To approach this question, we employed an extensive disulfide engineering approach in PAN, which allowed us to probe the conformations of the CC domains, their dynamics, and their role in regulating substrate translocation in the simplest proteasomal ATPase. Ultimately, this study was extended to determine what role the ATPases' CC domains play in proteasome function, not just whether they regulated the "global" ATP binding and hydrolysis pattern (See Chapter 2-Discussion for an in-depth discussion of the "global" binding and hydrolysis pattern).

Our results show that:

1) While PAN is a homohexamer its three identical coiled coil domains are actually in three different conformations, which was a complete surprise, and contradicts prior crystal structures of the $\mathrm{N}$-terminal fragments of PAN.

2) The different $\mathrm{CC}$ conformations in PAN do not cycle around the ring with ATP hydrolysis as one would initially expect. 
3) Two of the CC conformations exist in mutual exclusivity, with one conformation stabilizing a slow/resting state of the ATPase, and the other conformation stabilizing an activated state.

4) One of the $C C$ conformations shows a register shift that is four times larger than any other known register shift for a coiled-coil domain (e.g. 2 heptads vs. 1/2 heptad).

These results were important because they demonstrated that an ATPase with 6 identical subunits could assemble with an asymmetric conformation. This asymmetric conformation is similar to that found in the heterohexameric 26S, which indicates that these asymmetries arise from inter-subunit allostery, not from the evolutionary divergence of PAN into 6 different Rpt subunits in the 26S. In addition, it had been known for some time that substrate binding to the CC domains (PAN) or to ubiquitin receptors that are bound to the CC domains (26S), activates proteasome function. These studies elucidate the fundamental components of the underlying mechanism and describe how distinct conformations of the $\mathrm{CC}$ domains regulate proteasome activation. The two different conformational states in PAN that generate a resting or activated state are potentially analogous to resting and activated states of the $26 \mathrm{~S}$ proteasome that were found via cryo-EM (Matyskiela et al. 2013). These findings provided a path forward to understand how post-translational modification of the $\mathrm{CC}$ domains can regulate proteasome function. Therefore, this manuscript provides a mechanistic platform to better probe and understand how the $26 \mathrm{~S}$ proteasome is regulated, and of course it also uncovers fundamental roles of the $\mathrm{CC}$ domains in proteasome function. 
Finally, we also report and discuss this powerful method to analyze structural asymmetries in a homomeric protein complex with minimal structural perturbations, which has not been accomplished before and opened the door to an entire new approach to studying the function of the proteasomal ATPases (discussed further in Chapter 5).

The work presented in this chapter resulted in a $1^{\text {st }}$ author publication for me in Nature Communications. I designed, performed, and analyzed all experiments in this chapter with input from David M. Smith and Evan Brettrager.

Snoberger A, Brettrager E, Smith DM. (2018) "Conformational Switching in the coiledcoil domains of a proteasomal ATPase regulates substrate processing", Nature Communications (In-press).

This chapter has been reprinted here under Creative Commons Attribution 4.0 International License. 


\section{Abstract}

Protein degradation in all domains of life requires ATPase complexes that unfold proteins and inject them into compartmentalized proteolytic chambers. The proteasomal ATPases in eukaryotes and archaea have N-terminally conserved coiled-coil domains whose functional roles are not understood. In order to investigate potential mechanistic roles of these coiled-coil domains we focused on PAN-the archaeal proteasomal ATPases. By engineering specific disulfide crosslinks in PAN we found that its three identical coiled-coil domains simultaneously adopt 3 different conformations: 1) inregister and zipped, 2) in-register and partially unzipped, and 3) out-of-register. This conformational heterogeneity conflicts with the crystal structures of PAN's OB-coiledcoil fragment, which shows symmetric coiled-coil domain conformations. The specific structural conformations of PAN's coiled-coils resemble the conformational heterogeneity of the related coiled-coil domains in the $26 \mathrm{~S}$ proteasome's ATPases. Interestingly, while one coiled-coil's conformation never changes, even while unfolding substrates, conformational changes do occur in two of the coiled-coils, regulating the switching of PAN between resting and active states. These resting and active states of PAN appear to functionally mimic the similarly proposed states in the $26 \mathrm{~S}$ proteasome from cryo-EM. These findings thus build a mechanistic framework to understand the regulation of proteasome activity. 


\section{Introduction}

Across all domains of life, the proteasome is responsible for the majority of targeted protein degradation in the cell. Despite its crucial role in virtually every cellular process, surprisingly little is understood about how the proteasome operates. The main proteasome species in eukaryotes, the "26S" proteasome, is composed of 2 subcomplexes: the 19S (Regulatory Particle), which recognizes ubiquitinated substrates, and the 20S (Core Particle) which degrades substrates inside of its hollow interior. The 19S uses energy from ATP hydrolysis to unfold proteins and inject them into the $20 \mathrm{~S}$ for degradation. Cryo-EM structures of the $26 \mathrm{~S}$ reveal that the $19 \mathrm{~S}$ undergoes considerable conformational changes in response to substrate and/or ATPyS binding that appear to place the $26 \mathrm{~S}$ proteasome into a functionally competent conformation necessary for protein degradation ${ }^{1,2}$. These conformational changes seem to center around one of the 19S ATPase's coiled-coil (CC) domains (the "Rpt6/3" CC). The CC domains are composed of $\alpha$-helical extensions of the 19S's AAA+ ATPase subunits (Rpt1-6) that dimerize to form 3 CCs (Rpt1/2, Rpt6/3, and Rpt4/5 CCs). The CCs are intimately associated with many of the $19 \mathrm{~S}$ scaffolding subunits, substrate receptors, and deubiquitinases. For example, the Rpt $1 / 2$ CC is primarily associated with Rpn1, which serves as a docking station to coordinate multiple ubiquitin processing factors (e.g. the deubiquitinase USP14/Ubp6) at the $26 \mathrm{~S}$ proteasome $\mathrm{e}^{1,3,4}$. The Rpt6/3 CC is bound to the ubiquitin receptor Rpn13 via Rpn2 ${ }^{1,4-14}$. Additionally, after the substrate binds to the $26 \mathrm{~S}$, the Rpt4/5 CC binds to the ubiquitin receptor, Rpn10, as 
well as the proteasome's primary deubiquitinase, Rpn11 ${ }^{1}$. Thus, the CC domains physically connect substrate recruitment and ubiquitin processing to the unfolding machinery. This alone indicates that $\mathrm{CC}$ domains play fundamental roles in proteasome function. Mutagenesis of the $\mathrm{CC}$ domains and $\mathrm{CC}$ peptide competition studies further corroborate the functional importance of the $\mathrm{CC}$ domains, since most pertubations to any CC domain render the $26 \mathrm{~S}$ proteasome non-functional, leading to lethality ${ }^{15,16}$.

Several studies have shown that post-translational modifications of the CC domains affect ATPase and substrate processing activities, indicating that they are important for regulating proteasome function ${ }^{17-29}$. Early studies on the archaeal homolog of the 19S ATPases ("PAN") also found that partial truncations of its CC domains affected the rates of nucleotide hydrolysis and, surprisingly, even nucleotide specificity (full-length PAN could hydrolyze only ATP and CTP, whereas truncated PAN could hydrolyze ATP, CTP, ITP, GTP, TTP, and UTP) ${ }^{30}$. PAN's CC domains have also been shown to have chaperone activity (e.g. they could prevent protein aggregation, which the 195 also exhibits) ${ }^{31,32}$. Though ATP hydrolysis was not required for chaperoning, ATP binding did enhance the CC domains' chaperone activity ${ }^{30,33}$. These prior studies indicate that the CC domains are allosterically linked to the ATPase domains, yet no studies have shown how the CC domains function mechanistically to regulate protein degradation by the proteasome.

CCs are one of the most intensely studied and best understood tertiary structures and are made up of 2 or more $\alpha$-helices that wrap around one another in a "knobs into holes" fashion ${ }^{34}$. Dimeric, right handed CCs (the type found on proteasomal ATPases) 
have a repeating 7-residue consensus sequence termed a "heptad repeat" ${ }^{34,35}$. By convention these residues are named $a \rightarrow g$, where residues ' $a$ ' and 'd' are hydrophobic, ' $e$ ' and ' $g$ ' are charged, and the rest are typically polar (Fig. 4.1A) ${ }^{34}$. Dimerization is primarily stabilized by hydrophobic interactions between the hydrophobic ' $a$ ' and 'd' residues ${ }^{34,36}$. While CCs are often found in fibrous proteins and other rigid structures, some CCs undergo dynamic conformational rearrangements that regulate the functions of proteins ${ }^{37-41}$. For example, dynein's CC "stalk" undergoes a $1 / 2$ heptad registry shift (i.e. a shift in the sequence alignment between the $\alpha$-helices in a CC) to allosterically transmit long-distance signals in response to ATP binding or hydrolysis to modulate microtubule binding ${ }^{37}$. A theme among dynamic CCs is that they are rigid enough to help retain structure, but flexible enough to allow for movement of protein domains and/or send signals to distant domains via conformational rearrangements. In the present study, we examine the hypothesis that the proteasomal CCs must maintain a structure rigid enough to maintain subunit interactions, but also must be flexible enough to permit conformational changes within the ATPase ring to allow ATP-dependent substrate translocation to occur. In addition, we hypothesized that their position above the ATPase domains and their significant integration within the $19 \mathrm{~S}$ complex uniquely positions them to transmit allosteric signals between substrate binding components and the ATPase complex.

Much like the 19S ATPases, some studies suggest that the CC domains from PAN are also involved in substrate binding, although it is thought that PAN can achieve this without the use of additional substrate receptors that are found in the $19 S^{30,31,33,42-}$ 
${ }^{44}$. Because PAN effectively models the fundamental functions of proteasomal ATPases, we engineered disulfide crosslinks into the $\mathrm{CC}$ domains of PAN to probe the conformation of its $\mathrm{CC}$ domains and determine if CC sliding dynamics or conformational changes were necessary for proper ATPase function. We were surprised to find that although PAN is a homohexamer, its three identical CCs individually adopt three distinct conformations. Interestingly, although these three conformations are dependent on the presence of the AAA+ ATPase domains, their general conformations do not switch during ATP hydrolysis or substrate unfolding. However, local conformational changes within two of the CCs are required for PAN to switch between active and resting states, and these two states can be stabilized via specific disulfide crosslinks. These conformational states in PAN, which are regulated by its $\mathrm{CC}$ domains, may be functionally related to the "activated" and "resting" conformations previously described in the structures of the 19S ATPases 1,5,8,9,11,13,14,45. The allosteric linkages we establish also help demystify some of the previously confounding observations in early studies of PAN. 


\section{Results}

\section{Only 1 of PAN's 3 Coiled-Coils is in the Expected Conformation}

PAN is a homohexamer composed of a trimer of dimers. These dimers are held together by a coiled-coil (CC) domain composed of the $\mathrm{N}$-terminal $\alpha$-helical domains from two separate PAN subunits (Fig. 4.1B-C). While no high resolution structure of the full length PAN hexamer exists, there are crystal structures of hexameric CC-OB (ooiled-çoil - oligonucleotide/oligosaccharide b binding) domain fragment, named subcomplex I (PDB: 3H43). Although only a small portion of the CC domain is resolved in these structures (residues 74-150 in PDB:3H43 from Methanococcus jannaschii, and residues 57-134 in PDB: 2WG5 and 2WG6 from Archaeoglobus fulgidus) they clearly show that the $3 \mathrm{CC}$ domains are in register and fully formed up to the $\mathrm{OB}$ domain ending at residue M87 in PAN from M. jannaschii (the species used for this analysis) ${ }^{33,35}$ (Fig. 4.1C-D).

To determine if the $\mathrm{CC}$ domains in the full-length and active form of PAN are similarly in-register and zipped as observed in the crystal structure, and to determine whether this conformation is functional, we started by mutating the most proximal hydrophobic 'd' residue, M87, to a cysteine (M87C, or "87C" mutation) (Fig. 4.2A). Because it has been shown that Methionine and Cysteine have similar stabilities in the 'd' position of CCs, mutation alone should not significantly affect the stability of the CC ${ }^{46}$. Under oxidizing conditions, disulfide crosslinks will form almost instantaneously when

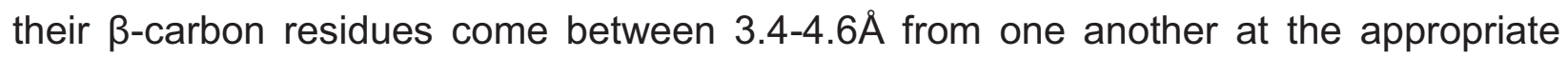
angle (pseusobond angles of: $\left.60^{\circ}<\theta_{\mathrm{ij}}, \theta_{\mathrm{ji}}<180^{\circ}, 0^{\circ}<\left|\theta_{\mathrm{ij}}-\theta_{\mathrm{ji}}\right|<105^{\circ}\right)^{47}$, and the 


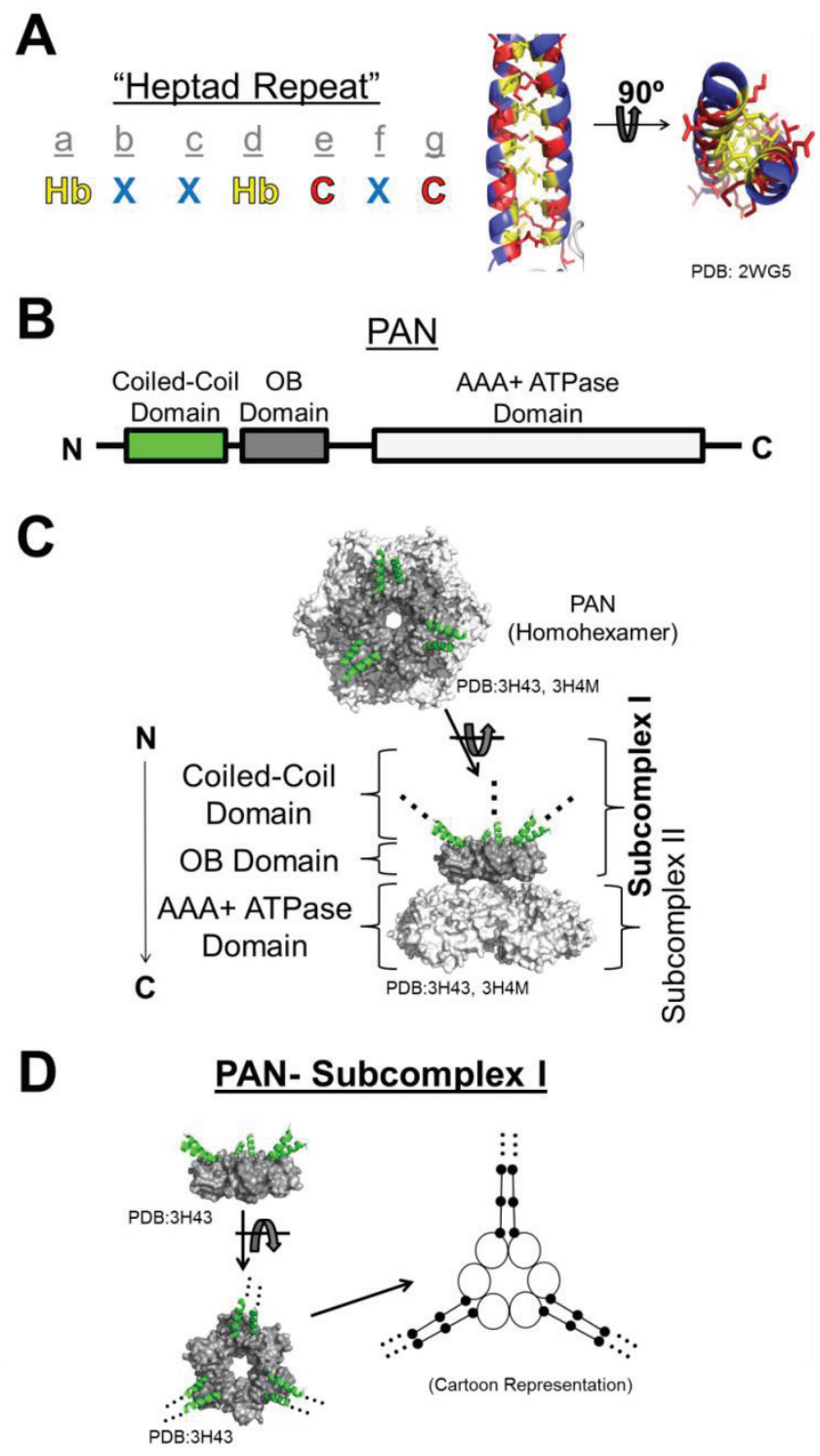

Figure 4.1: PAN Contains 3 Right Handed, Dimeric Coiled Coils on its N-terminus.

A) Right-handed, dimeric coiled-coils (CCs) contain a 7-residue repeating consensus sequence (i.e. "heptad repeat") where residues 'a' and 'd' are hydrophobic ( $\mathrm{Hb}$, yellow), 'e' and ' $g$ ' are charged $(C$, red), and the rest are typically polar $(X$, blue). $\mathrm{Hb}$ residues pack on the interior of the $\mathrm{CC}$ and form the main stabilizing interactions (PDB: 2WG5). B) The proteasome activating nucleotidase (PAN) from archaea contains an N-terminal CC domain, followed by an oligonucleotide/oligosaccharide binding (OB) domain and a AAA+ ATPase domain C) Crystal structures of the N-terminus of PAN (subcomplex I, or CC-OB domain) show PAN's 6 identical subunits in a symmetric ring (PDB: $3 \mathrm{H} 43$ ). The AAA+ ATPase domain (PDB: $3 \mathrm{H} 4 \mathrm{M}$, light gray) also forms a hexameric ring underneath the OB domain (dark gray). 3 dimeric $C C$ domains protrude from the OB domains (green $\alpha$-helices). The OB and ATPase domains domain are shown as a spacefill and the CC domains are shown as helices (PDB: 3H4M, 3H43). D) The structure of PAN's CC-OB domain (subcomplex I) shown from a "side" and "top" view. An arrow points to the "cartoon" version of the CC-OB domain complex. The backbone of the CC domains are depicted as "unwound" sticks, while the black dots represent the inward facing hydrophobic residues. PDB: $3 \mathrm{H} 43$. 

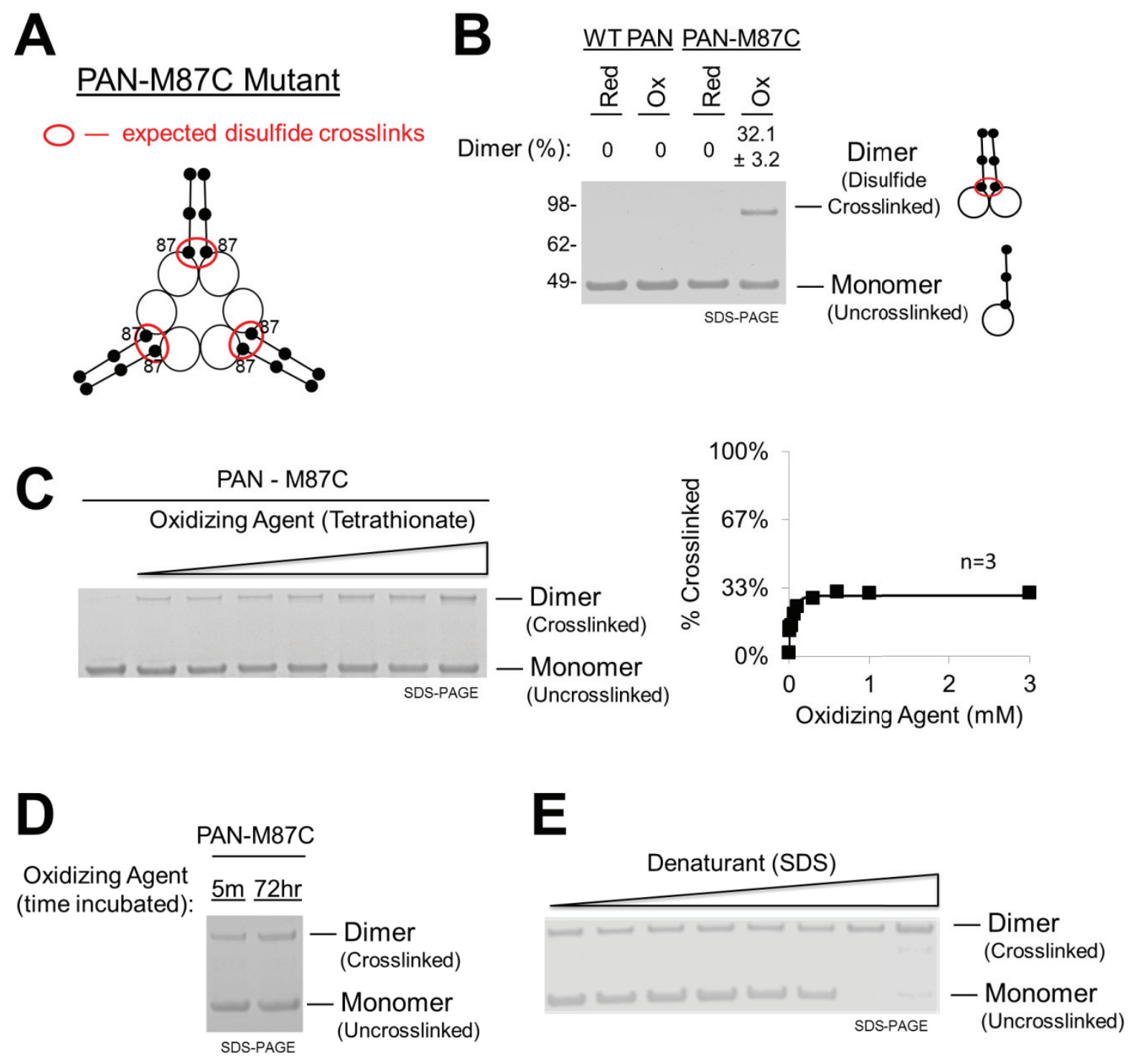

Figure 4.2: PAN's Coiled-Coils do not Adopt Symmetrical Conformations

A) Cartoon representation of the PAN-M87C mutant CC-OB domains (based on PDB: 3H43). The PAN-M87C mutant contains a cysteine in place of methionine at the CC domains most proximal hydrophobic residue. These cysteines are expected to form a disulfide crosslink based on measured $\beta$-carbon distances in its crystal structure (PDB: 3H43). B) Representative nonreducing SDS-PAGE and coomassie staining of WT PAN and PAN-M87C under reducing (1mM DTT) and oxidizing (1 $\mathrm{mM}$ tetrathionate) conditions followed by desalting (See methods for details). The mean percentage of dimer with standard deviations is indicated at the top of the gel $(n=10)$. C) Experiment with PAN-M87C similar to B, but with a dose response with oxidizing reagent (tetrathionate) prior to desalting and SDS-PAGE. The quantification of $\%$ crosslinked versus the concentration of tetrathionate is also shown on the right. Error bars are shown $(n=3)$, but are smaller than the data points. D) Representative gel showing the amount of PAN-M87C crosslinking after $5 \mathrm{~min}$ or $72 \mathrm{hrs}$ of incubation with tetrothionate $(n=3)$. E) PAN-M87C was incubated in $1 \mathrm{mM}$ tetrathionate, and increasing amounts of denaturant (SDS: $0.00006 \%$, $0.0006 \%, 0.003 \%, 0.006 \%, 0.03 \%, 0.06 \%, 0.3 \%$, and $0.6 \%$ ) were added prior to desalting and SDS-PAGE. At higher levels of SDS, $>95 \%$ crosslinking was observed. A representative gel is shown ( $n=3)$. See Fig S6 for validation of quantitative SDS-PAGE analysis of PAN. 
cysteine residues cannot crosslink if these conditions are not met. Therefore, based on PAN's CC-OB domain structure (PDB: $3 \mathrm{H} 43$ ) the M87C mutation should allow all of the CCs to form 87C-87C disulfide crosslinks under oxidizing conditions (Fig. 4.2A). We incubated WT PAN or PAN-M87C mutants with oxidizing reagent, tetrathionate (TT), or the reducing agent dithiothreitol (DTT) for 1 hour at room temperature and then separated them on a non-reducing SDS-PAGE gel. Based on the crystal structure we expected to find that WT PAN would run as monomers and the oxidized M87C would run as only dimers. WT PAN ran as monomers as expected, but we were surprised to find that only $32.1 \pm 3.2 \%(n=10)$ of PAN-M87C ran as dimers (with the remaining $67.9 \%$ running as monomers) (Fig. 4.2B). We then sought to determine why PANM87C did not completely crosslink all subunits as initially expected. The incomplete crosslinking was not due to perturbation of PAN's hexameric quaternary structure, since the oxidized form of M87C PAN formed hexamers via native-PAGE (Fig. S4.1A) and retained their normal WT-like functions, including their ability to activate gate-opening in the 20 S proteasome (Fig. S4.1B) and even hydrolyze ATP and unfold proteins (discussed in detail later, Fig. 4.6A \& B).

Three other possibilities can explain incomplete crosslinking of the M87C mutant: 1) An incomplete oxidation reaction, 2) PAN hexamers could exist in two different conformational populations, one that is "crosslinkable" and another that is not, or 3) the three CC domains in PAN exist in different conformations and only one of the three (i.e. $33 \%$ of total) exists in a conformation that permits M87C crosslinking. In order to test possibility \#1, we conducted a dose-response with oxidizing reagent, tetrathionate, to 
determine whether the $87 \mathrm{C}-87 \mathrm{C}$ crosslink was saturable. M87C crosslinking saturated at $31.6 \pm 0.8 \%$ dimer formation (Fig. $\mathbf{4 . 2 C}$ ), indicating that all cysteines within proximity to one another had successfully crosslinked, thus excluding possibility \#1. Regarding possibility \#2, we can expect that if PAN exists in an equilibrium between two different conformational populations (e.g. "crosslinkable" and "uncrosslinkable"), that given enough time, there should be a conversion of some, if not all, CCs to a "crosslinkable" state. To test possibility \#2 we thus performed a crosslinking reaction at saturating levels of oxidizing reagent for up to 72 hours. We observed $28.9 \%$ crosslinking after only 5 minutes and little additional crosslinking was observed at longer times (34.3\% after 72 hours) (Fig. 4.2D). This demonstrates that PAN is not in equilibrium between two different crosslinkable/uncrosslinkable states, which rules out possibility \#2. To test possibility \#3 and ask if PAN's three CC domains are restrained into three different conformations we added a denaturant (SDS) to determine if "loosening" PAN's quaternary structures could increase the amount of crosslinking. We found that increasing concentrations of denaturant resulted in an increase in $87 \mathrm{C}-87 \mathrm{C}$ crosslinking up to $96.3 \pm 4.9 \%$ dimers (Fig. 4.2E). This suggests that the lack of $87 \mathrm{C}-87 \mathrm{C}$ crosslinking in $\sim 67 \%$ of PAN-M87C's CC domains is attributed to conformational restraints that prevent the $\mathrm{M} 87 \mathrm{C}$ residues from coming into close proximity.

Taken together, we conclude that the $32.1 \pm 3.2 \%$ crosslinking observed in PAN's M87C residue is due to a single CC domain adopting a conformation similar to that found in the $\mathrm{CC}-\mathrm{OB}$ domain crystal structure, while the other two $\mathrm{CC}$ domains cannot access this same conformational state and thus $87 \mathrm{C}-87 \mathrm{C}$ crosslinking does not 
occur in these CCs. These data therefore indicate that although PAN has 3 identical CC domains, they must be conformationally asymmetric. Because these disulfide crosslinking experiments (which were done on full length PAN) did not agree with the CC-OB domain crystal structure, we hypothesized that we could achieve $100 \% 87 \mathrm{C}$ $87 \mathrm{C}$ crosslinking after separating the ATPase domain from the CC-OB domain. The first $\mathrm{CC}-\mathrm{OB}$ domain crystal structure was generated via partial proteolysis to remove the ATPase domain. Therefore, we subjected our PAN-M87C mutant to similar partial proteolysis conditions (Fig. 4.3A) and purified the two sub-complexes of PAN by gel filtration as previously described by Zhang et al. 2009 (Fig. 4.3B) ${ }^{35}$. We analyzed both subcomplexes via SDS-PAGE and found that Subcomplex I (F3) contained an $\sim 8 \mathrm{kDa}$ fragment (the expected size of a single CC-OB domain), and a $\sim 16 \mathrm{kDa}$ fragment (the expected size of a CC-OB dimer) (Fig. 4.3C), while subcomplex II (F2) predominantly contained a $\sim 30-35 \mathrm{kDa}$ fragment, the expected size of a single AAA+ ATPase domain (Fig. S4.2). We further analyzed full-length PAN (F1) and subcomplex I (F3) since these fragments contain a $\mathrm{CC}$ domain (whereas subcomplex II contains only the AAA+ ATPase domain) ${ }^{35}$. Partial proteolysis of the sample resulted in a high background, but the full length (or near full length) fraction (F1) still clearly showed PAN dimers and monomers (Fig. 4.3C, F1-top of gel Ox lane) just as we observed in Fig. 4.2B. However, $\sim 100 \%$ of the CC-OB domain fragment (Fig. 4.3C, F3-bottom of oxidized lane) was crosslinked and ran as dimers under oxidizing conditions. Even a large fraction of the reduced form of the $\mathrm{CC}-\mathrm{OB}$ fragment crosslinked, likely in the gel, due to the necessary non-reducing conditions (Fig. 4.3C, F3-bottom of reduced lane). This demonstrates that upon removal of the AAA+ ATPase domains, all of the CC-OB 


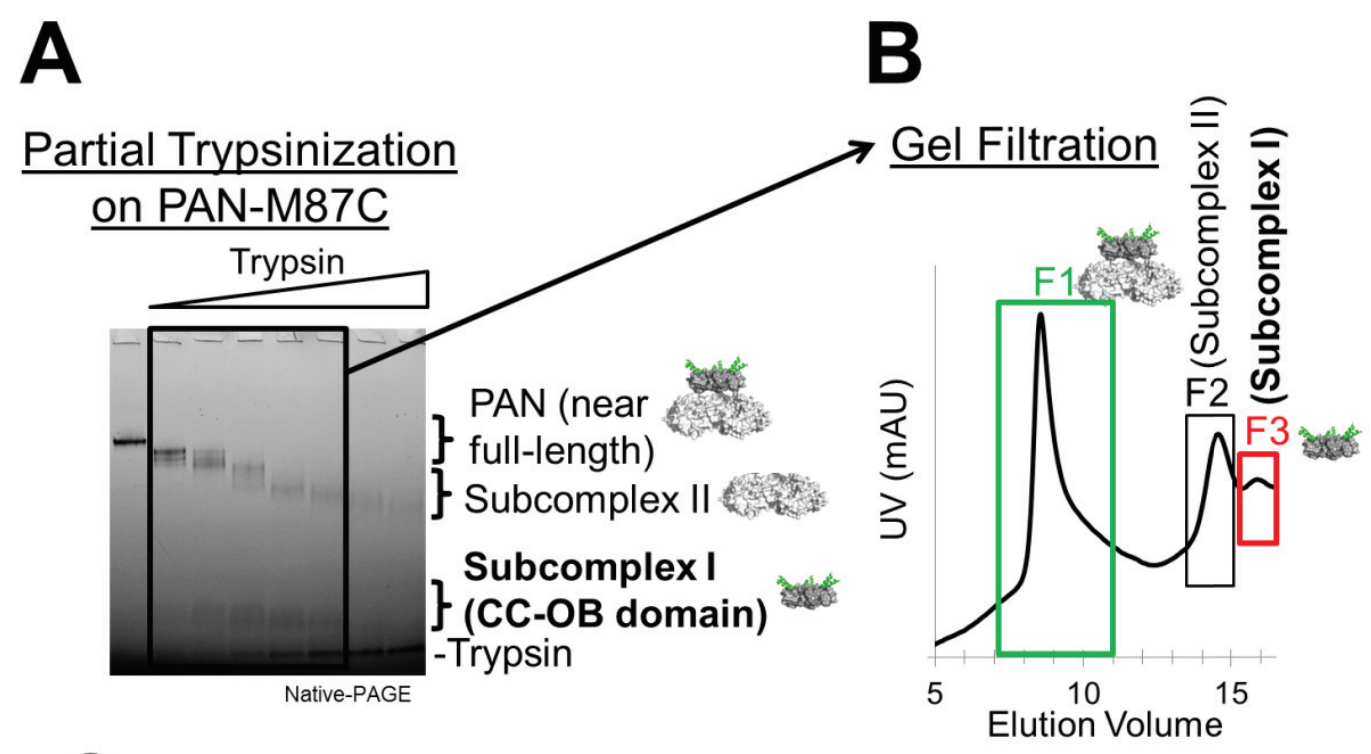

Disulfide Crosslinking of

PAN Fragments

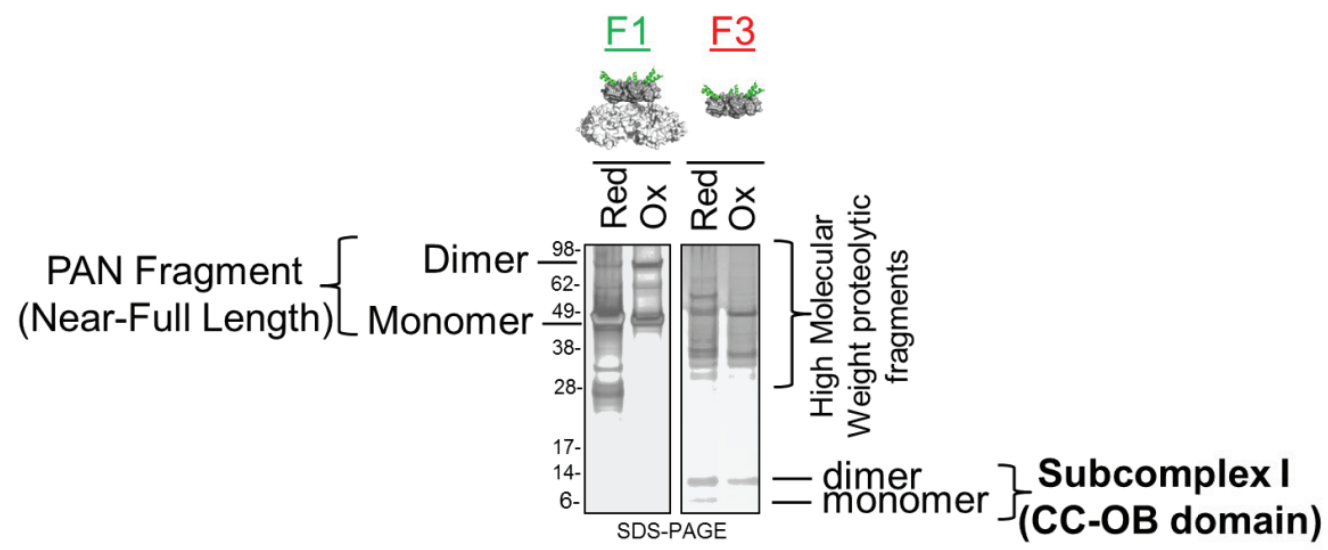

Figure 4.3: PAN's ATPase Domains Induce a Conformational Asymmetry in its CoiledCoils

A) PAN M87C was incubated with increasing amounts of trypsin and analyzed by native PAGE to generate two subcomplexes of PAN ${ }^{35}$. Black box indicates fractions that were pooled for gel filtration in (B). B) Gel filtration was conducted on pooled fractions from (A). 3 main peaks were observed that correspond to molecular weights of near-full-length PAN, Subcomplex II (AAA+ ATPase domain), and Subcomplex I (CC-OB domain). C) Fractions 1 and 3 from (B) were incubated under reducing (1 $\mathrm{mM}$ DTT) or oxidizing conditions (1 $\mathrm{mM}$ Tetrathionate), desalted, and analyzed by SDS-PAGE and Silver Staining. 
domains are able to fully form the $87 \mathrm{C}-87 \mathrm{C}$ crosslink, consistent with the crystal structures of this fragment. Since two independent methods that disrupt conformational restraints (i.e. SDS and partial proteolysis) both allow full crosslinking, we conclude that in full-length PAN, only $\sim 1 / 3$ of PAN's CC domains are crosslinkable because only 1 of its $3 \mathrm{CCs}$ are zipped and in-register at this proximal position (implying that PAN's other 2 CCs must adopt different conformations, discussed in detail below). We will call the inregister and zipped CC "C1" (for CC conformation 1).

\section{Nucleotides Have Little Effect on Coiled-Coil conformation}

All of the described crosslinking experiments to this point were performed in the absence of nucleotides. Given that the AAA+ ATPase domains seem to allosterically restrict the conformation of PAN's CC domains, we questioned whether the nucleotidebound state could regulate PAN's CC domains. In order to test this, we performed crosslinking reactions for 1 hour at room temperature in the Apo (no nucleotide), ADP, ATP, and ATPYS state (Fig. S4.3A). In addition, we also confirmed, via mass spectrometry, that under oxidizing conditions, an $87 \mathrm{C}-87 \mathrm{C}$ crosslink occurred in the Apo state (Fig. S4.3B). No difference in crosslinking was observed when nucleotides were bound, except for a modest decrease in crosslinking in the high ATPYS state (Fig. S4.3A), which has previously been shown to force PAN into an unnatural 4-ATP-bound state with suboptimal activity ${ }^{48}$. It is important to note that after 1 hour at room temperature, reactions are expected to have gone to completion, so these experiments could not resolve whether the binding of nucleotides caused differences in PAN-M87C's time-to-crosslinking. Therefore, we analyzed the timecourse of PAN-M87C crosslinking 
immediately after addition of oxidizing agent. Since crosslinking occurs very quickly, we conducted this experiment at $-17^{\circ}$ in order to attempt to resolve and compare the time-to crosslinking with various nucleotides added (see methods for details). The rate of M87C crosslink formation with $2 \square \mathrm{M} \mathrm{ATP} \square \mathrm{S}$ was not discernably different from crosslink formation in the Apo state. However, we did observe a slight reduction for the rate of crosslinking in the presence of ADP (Fig. S4.3C). Therefore, saturating ADP levels appear to slightly disorganize the $\mathrm{C} 1$ conformation (i.e. slows the rate of M87C crosslinking), but the binding of ADP does not completely restrict PAN from entering the C1 conformation.

\section{PAN's Coiled-Coils Adopt Distinct Conformations}

Since PAN is a homohexamer it was a surprise to find that PAN's 3 CC domains are not in the same conformation. These results indicate that the other $2 \mathrm{CC}$ domains in PAN must be in different conformational states, perhaps unzipped or out of register. To test this possibility, we began engineering other cysteine mutants in PAN that could allow crosslinking of other potential CC conformational states. PAN's CCs extend from residue 87 to $\sim$ residue 50 (with 90\% confidence) (Fig. 4.4A). In order to determine whether PAN's CCs may be "unzipped" proximally and then "rezipped" distally (e.g. see Fig. 4.4C), we systematically mutated each individual hydrophobic 'a' and 'd' residue in PAN's CC to a cysteine starting from the proximal end and moving to the distal end. As with other CC-containing proteins, PAN's 'a' residues mutated to cysteine did not easily form disulfide bonds (Fig. S4.4A), likely due to the unfavorable $\mathrm{C} \alpha-\mathrm{C} \beta$ bond angle of the 'a' residues ${ }^{50}$. Thus, we limited our analysis to hydrophobic 'd' residues in PAN. Using 

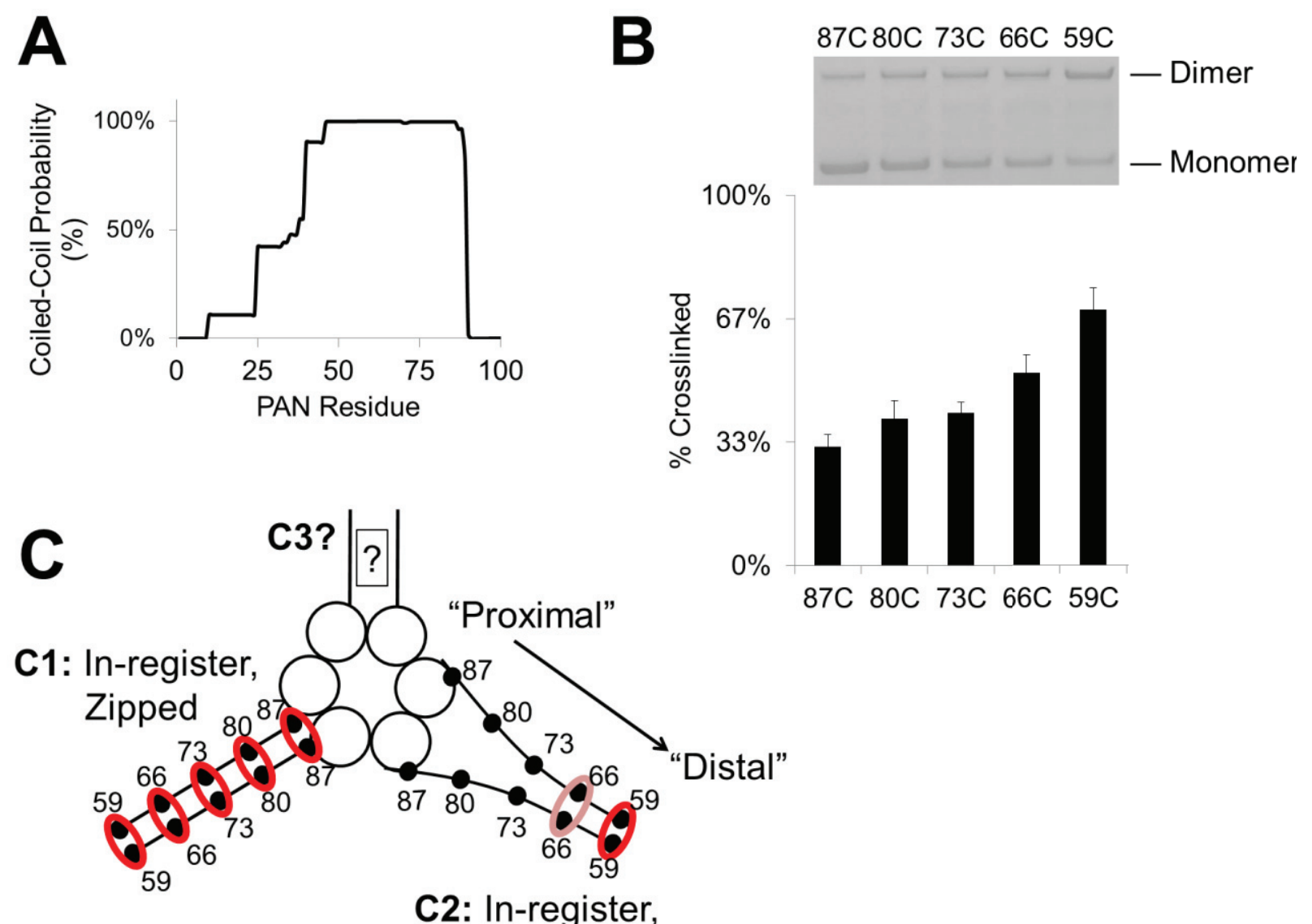

Unzip/Rezip

- expected disulfide crosslinks

Figure 4.4: One of PAN's Three Coiled-Coils is Partially Unzipped

A) Prediction of PAN's CC domains via Parcoils software ${ }^{56}$. B) Oxidation followed by desalting and SDS-PAGE of the indicated 'd' residue point mutants of PAN along the length of the CC. In this and following figures we indicate the cysteine mutations by listing the residue number followed by a "C", (e.g. residue M87 mutated to cysteine is denoted as "87C"). Quantification of \% crosslinked is shown below in bar graph form as means \pm standard deviations, $n=10$. $p$-values for all points relative to $87 \mathrm{C}$ were $<0.001$. C) Summary model of in-register disulfide crosslinks that can occur in PAN's C1 and C2 CCs. Red circles represent crosslinked residues, pink circles indicate partial crosslinking. 
the same protocols we used for the M87C experiments, we observed a gradual increase in disulfide crosslinking formation from proximal to distal CC residues (Fig. 4.4B) with the $59 \mathrm{C}$ mutation $\left(5^{\text {th }}\right.$ heptad) resulting in $69 \pm 5.9 \%$ crosslinking (59C-59C crosslink) (Fig. 4.4B). Based on the prior experiments we expect that all 5 heptads in C1 (crosslinked by M87C) are in register and zipped, and thus contribute $\sim 33 \%$ crosslinking to these experiments, so every 'd' residue cysteine mutation should also crosslink in the C1 CC (Fig. 4.4C). Therefore, the additional crosslinking that is observed (up to $\sim 69 \%$ in the more distal cysteine mutants likely comes from a $2^{\text {nd }}$ in-register $\mathrm{CC}$ that is able to crosslink distally but not proximally, we call this $\mathrm{CC}$ conformation $\mathrm{C} 2$. Thus, the $\%$ of crosslinked PAN changes from $1 / 3$ (87C-87C crosslink) to $2 / 3$ at $59 \mathrm{C}$ (59C-59C crosslinks) (Fig. 4.4B-C). It therefore appears that $\mathrm{C} 1$ is in register and fully zipped while $C 2$ is in register but is unzipped at its proximal residues (Fig. 4.4C).

Since we never found a single cysteine mutation (which probes for in-register CCs only) in PAN that could simultaneously crosslink all three of its CCs, the next step was to determine if the $3^{\text {rd }} \mathrm{CC}$ domain was in an out-of-register conformation or potentially unstructured. We therefore systematically generated double mutants that could crosslink both in register and out of register CC conformations of different sized registry shifts (e.g. see Fig. 4.5A). To analyze whether PAN's $3^{\text {rd }} \mathrm{CC}$ conformation is slidden by less than 1 heptad, we generated double cysteine mutations with residue 87 (the first hydrophobic ' $d$ ' residue) plus one of each of the residues in the $1^{\text {st }}$ heptad $(86 \rightarrow 81 \mathrm{C})$ (Fig. S4.4B). Such mutants can allow for crosslinking of both in register and out of register conformations (e.g. an $87+86 \mathrm{C}$ mutant could allow for an in-register $87 \mathrm{C}$ - 
$87 \mathrm{C}$ and $86 \mathrm{C}-86 \mathrm{C}$ crosslink, or it could allow for an out-of-register $87 \mathrm{C}-86 \mathrm{C}$ crosslink). Therefore, if a registry shift is present, we expect approximately $2 / 3$ crosslinking under oxidizing conditions ( $1 / 3$ from $\mathrm{C} 1$ and $1 / 3$ from $\mathrm{C} 3$ ). However, none of these mutations had significant crosslinking above that of their single mutant controls (e.g. the $87+86 \mathrm{C}$ mutant did not crosslink more than the single $87 \mathrm{C}$ and $86 \mathrm{C}$ mutants combined) (Fig. S4.4C). This result ruled out the possibility a registry shift of less than one heptad.

To test the possibility of register shifts of greater than one heptad we generated cysteine mutants that could crosslink under conditions of a 1,2, 3, or 4 heptad slide (Fig. 4.5A). Interestingly, double mutations engineered into the first 3 heptads that would allow crosslinking of a single full heptad slide (i.e. 1 heptad slide mutants; Fig. 4.5A) all resulted in less crosslinking than their single mutant controls (Fig. 4.5B), suggesting that mutation of more than $2-$ ' $d$ ' residues in a row may disrupt local CC stability. However, if a 1- heptad slide did occur (even briefly), we expect that disulfide crosslinking would have stabilized this 1-heptad slidden conformation. So, even though it's unlikely, we cannot completely rule out that PAN's $3^{\text {rd }} \mathrm{CC}$ adopts a 1-heptad slide, since the controls produce less than the expected crosslinking in these particular double mutants. We next engineered mutants that would stabilize full heptad slides of 2 , 3 , or 4 heptads (Fig. 4.5A-right 3 panels). Mutants engineered to crosslink 2-heptad slides did crosslink more than their single mutant controls, (Fig. 4.5C-left two gels), but mutants engineered to detect 3-heptad $(80+59 \mathrm{C})$ and 4-heptad $(87+59 \mathrm{C})$ slides did not show additional crosslinking (Fig. 4.5C-right two gels) ruling out 3-heptad and 4-heptad registry shifts. Since crosslinking of PAN $(87+73 \mathrm{C})$ resulted in additional crosslinking 

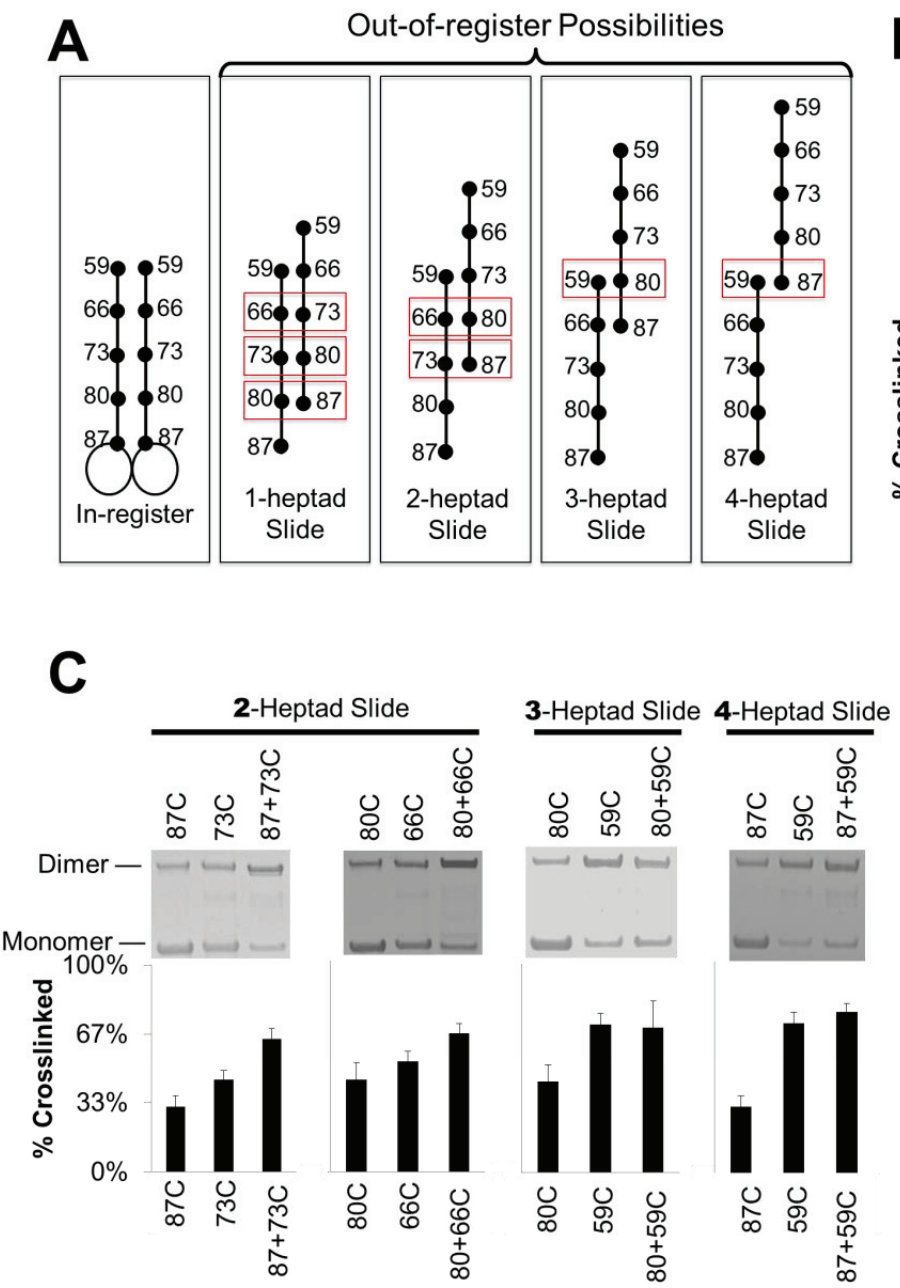

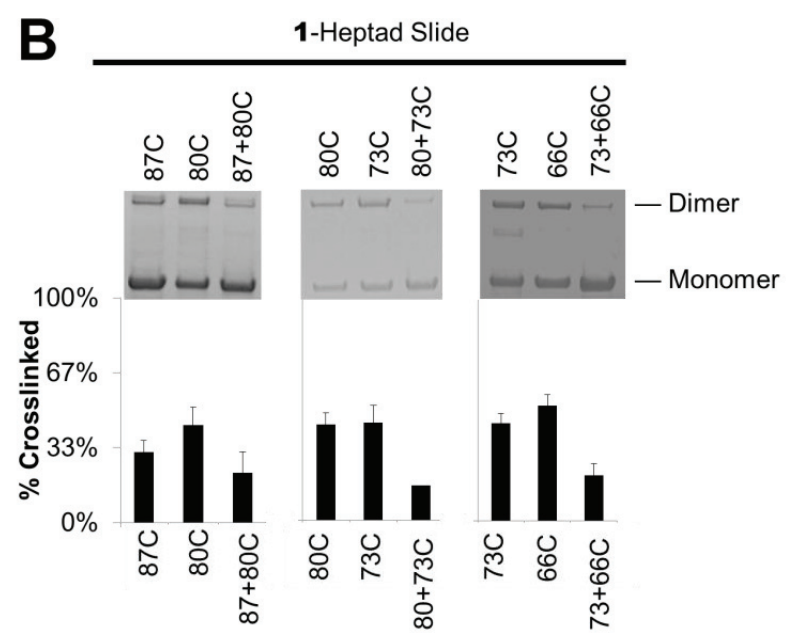

D

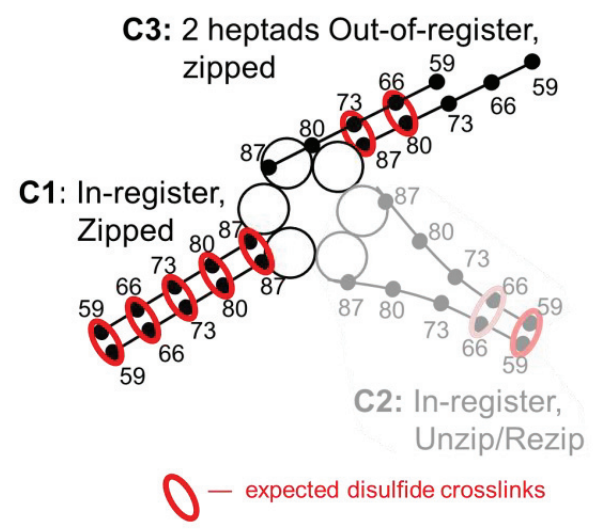

Figure 4.5: One of PAN's Coiled-Coils is 2 Heptads Out-of-Register

A) Illustration of possible CC register shifts. The boxed areas are expected crosslinks between 'd' residues mutated to cysteines, assuming full "zipping" at these residues. B) The indicated PAN mutants that allow for crosslinking of one-heptad slide (double mutants) and their single mutant controls were subjected to crosslinking ( $1 \mathrm{mM}$ Tetrathionate) and run on SDS-PAGE for analysis. $\%$ crosslinked is quantified in bar graph below. C) Same as B, but using the indicated mutants allowing for crosslinking of 2, 3, and 4 heptad slides. Only 2-heptad slides show additional crosslinking in their double mutant compared to single mutant controls. Bar graphs are means \pm standard deviation of 3 or more independent experiments $(n \geq 3)$. D) Summary model of the crosslinks that can occur in PAN's C1 and C3 CCs (the crosslinks that form in the C2 CC are also lightly pictured here). Red circles represent crosslinked residues. 
over their single mutant controls $(66.5 \pm 4.3 \%$ total crosslinking) (Fig. 4.5C) this indicates a two-heptad slide could be detected in this mutant. In the $73 \mathrm{C}$ single mutant, $\sim 33 \%$ crosslinking is expected to arise from the $\mathrm{C} 1$ conformation (73C-73C crosslink), with $\sim 7 \%$ crosslinking coming from the $\mathrm{C} 2$ conformation (Fig. 4.4B). We thus hypothesized that in the $87+73 \mathrm{C}$ double cysteine mutant, somewhere between $27-34 \%$ crosslinking is contributed by the 2 heptad out-of-register CC due to an $87 \mathrm{C}-73 \mathrm{C}$ crosslink, (called conformation "C3"). To further support this hypothesis, we engineered a second mutation capable of capturing a 2-heptad slide, " $80+66 \mathrm{C}$ ", which is identical to the $87+73 \mathrm{C}$ mutation but one heptad more distally located (forming an $80 \mathrm{C}-66 \mathrm{C}$ crosslink). We observed $72 \%$ crosslinking, consistent with the level of crosslinking observed in the $87+73 \mathrm{C}$ mutation (Fig. 4.5C) thus also confirming the 2-heptad slide with a different double mutant. So, we have generated 2 crosslinkable mutants $(87+73 \mathrm{C}$ and $80+66 \mathrm{C}$ ) that are capable of simultaneously crosslinking the $\mathrm{C} 1$ conformation (87C$87 \mathrm{C} / 73 \mathrm{C}-73 \mathrm{C}$, and $80 \mathrm{C}-80 \mathrm{C} / 66 \mathrm{C}-66 \mathrm{C}$ crosslinks) and the C3 conformation (87C-73C and $80 \mathrm{C}-66 \mathrm{C}$ crosslinks). Both of these mutants were hexameric by native page (Fig. S4.1A) and had similar 20S gate opening capacity (Fig. S4.1B), meaning these mutants are functional and have the expected quaternary structure. These data indicate that one of PAN's CCs natively adopts a full 2-heptad slide (C3-conformation). To our knowledge, in all other proteins where the structure of out-of-register CCs are available, sliding only occurs over $\sim 1 / 2$ heptad ( $\sim 4-5$ residue slide), and to date, such an extensive 2 heptad registry shift has not been reported ${ }^{37-40}$. 


\section{Coiled-Coil Conformations Regulate ATPase Rate \& Substrate Processing}

The above results indicate that the AAA+ ATPase domains of PAN impose structural restraints on PAN's 3 CC domains such that they adopt at least 3 distinct conformations: C1 (in-register, zipped), C2, (partially unzipped), and C3 (2 heptads outof-register). We hypothesized that this ATPase to CC domain allosteric communication should also work in the reverse direction. In other words, the CC domains, which are associated with substrate binding, may allosterically regulate the activity of the distant ATPase domains. To test this hypothesis, we sought to determine ATP hydrolysis kinetics of WT PAN and the cysteine mutants that crosslink the various combinations of C1, C2, and C3 conformations that we have identified. Under reducing conditions, WT PAN performed the same as previous observations and oxidizing conditions did not significantly alter its kinetics (see Table S4.1 for values, Fig. 4.6A and S4.3). We then determined the rates of ATP hydrolysis for the CC mutants in the reduced (uncrosslinked) and oxidized (crosslinked) conditions. Under reducing conditions all of the mutants hydrolyzed ATP with rates similar to WT (Fig. 4.6A, white bars). Interestingly, under oxidizing conditions, the 87C (crosslinked C1) showed normal ATPase activity and 59C (crosslinked C1 and C2) had a 25\% increase in its Vmax for ATPase activity compared to its reduced control after normalizing to WT PAN $(p<0.001)$ (Table S2). The lack of functional impact from crosslinking was an unexpected result, so we also verified by SDS-PAGE that the anticipated crosslinks were indeed present at the expected levels from the PAN that was previously used in the same rate reactions. Moreover, we also repeated this same experiment while monitoring PAN's ability to 

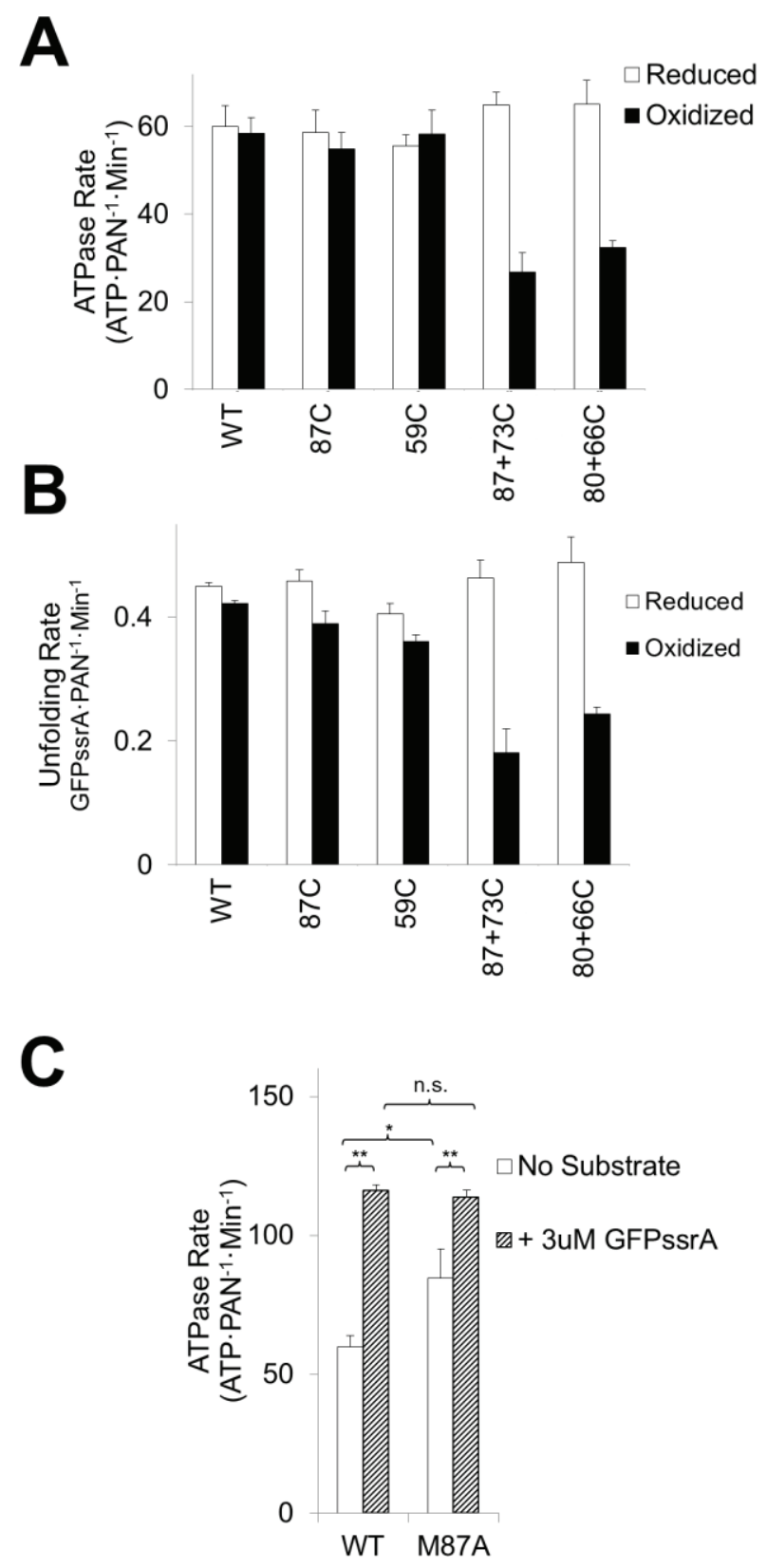

Figure 4.6: Some Coiled-Coil Conformations Regulate PAN's Activity.

A) ATPase activity of the indicated PAN mutants under reducing and oxidizing conditions with saturating $2 \mathrm{mM}$ ATP. ATPase activity is determined using an ATP regenerating system coupled to $\mathrm{NADH}$, whose absorbance is monitored in real time. B) Unfoldase activity of the indicated PAN mutants was measured under reducing and oxidizing conditions using saturating substrate (GFPssrA, 3uM) with saturating ATP (2mM). The rate of GFP unfolding was determined by assaying its loss of fluorescence in the presence of the indicated PAN and the 20S proteasome. C) ATPase activity of WT PAN and PAN-M87A in the absence or presence of saturating (3uM) substrate. Bar graphs values are means \pm standard deviation of 3 or more independent experiments $(n \geq 3) .{ }^{*}-P<0.05,{ }^{* *} P<0.001$, n.s. - not significant 
unfold GFP-ssrA (Fig 4.6B) and we found similar results as when we followed ATPase activity: PAN functioned well even with crosslinked $\mathrm{C} 1$ and $\mathrm{C} 2$ conformations. While the 59C-59C crosslink covalently "locks in" the C1 and C2 CCs, this crosslink is located toward the $\mathrm{N}$-terminal end of the CC domain, which could therefore still allow for conformational changes to occur in the C-terminal side of the CC, which is located adjacent to the $\mathrm{OB}$ domain. However, the $87 \mathrm{C}-87 \mathrm{C}$ crosslink is on the most $\mathrm{C}$-terminal residue in PAN's CC and stabilizes the CC domain directly adjacent to the OB domain. The fact that PAN's activity is unaltered in the $87 \mathrm{C}-87 \mathrm{C}$ crosslink demonstrates that PAN's C1 CC can remain in-register and zipped throughout the entire ATP hydrolysis and substrate unfolding cycle. To further test this possibility, we mutated the M87 residue to an alanine (M87A), which is a conserved mutation but is less stable at this ' $d$ ' position than a methionine or cysteine (i.e. M87A will slightly and locally destabilize the C1 conformation of the CC) ${ }^{46}$. It is known that WT PAN has repressed ATPase activity in the absence of substrate, and that the addition of saturating amounts of substrate causes "activation" of its ATPase activity. Intriguingly, the repressed state (no substrate) of PAN M87A hydrolyzed ATP faster than WT (Fig. 4.6C-white bars), but had the same ATPase activity as WT in the activated state (substrate-bound) (Fig. 4.6C-hatched bars). Therefore, a stable $\mathrm{C} 1$ conformation at the $\mathrm{M} 87$ residue is necessary to properly repress PAN in the absence of substrate, thus allowing for substrate mediated ATPase activation to occur.

To test the functional effects of crosslinking the $\mathrm{C} 3 \mathrm{CC}$ conformation we also determined the rates of ATP hydrolysis for the $87+73 \mathrm{C}$ and $80+66 \mathrm{C}$ mutants, which 
both crosslink the $\mathrm{C} 1$ and $\mathrm{C} 3$ conformations. Interestingly, under only oxidizing conditions, we observed a substantial reduction in PAN's ATPase activity in both of these crosslinked variants (Fig. 4.6A). Similar results were also found when we performed the same experiments but assayed protein unfolding activity (Fig. 4.6B). Since crosslinking the $\mathrm{C} 1$ conformation has no effect on activity, and since crosslinking C1+C3 lowers PAN's activity, these data indicate that stabilizing the $\mathrm{C} 3$ conformation by crosslinking stabilizes a functional, but low activity state of PAN. This result suggests that crosslinking the C3 state results in the slowing of some step in the ATP hydrolysis cycle (ATP binding, ATP hydrolysis/phosphate leaving, or ADP off rate), but does not have an effect on the mechanochemical coupling of ATP hydrolysis to substrate unfolding previously observed for proteasomal ATPases ${ }^{51}$. While PAN $87+73 \mathrm{C}$ alone in the reduced state had a slightly lower $V \max \left(42 \pm 1 \mathrm{ATP} \cdot \mathrm{PAN}^{-1} \cdot \mathrm{Min}^{-1}\right)$, it had a similar $\mathrm{Km}$ as WT PAN (556 $\pm 29 \mathrm{mM})$ (Table S4.1, Fig. S4.5). However, when this $87+73 \mathrm{C}$ mutant was crosslinked under oxidizing conditions, we observed such a severe impairment in ATP hydrolysis that we could not achieve saturation with ATP, indicating a large increase in the $\mathrm{Km}$ for ATP hydrolysis (> $3000 \mu \mathrm{M})$ (Table S4.1, Fig. S4.5). We also observed a $63 \%$ reduction in ATPase and a $64 \%$ reduction in unfoldase activity in the other 2-heptad slidden mutant $(80+66 \mathrm{C})$ at high ATP (Fig. 4.6A-B). These results demonstrates that trapping one of PAN's CCs in the C3 conformation dramatically alters the kinetics of ATP hydrolysis, most likely due to a specific allosteric mechanism that is regulated by the $\mathrm{C} 3 \mathrm{CC}$ conformation. 


\section{PAN's Coiled-Coils Toggle Between "Activated" and "Resting" Conformations}

Given that our $87 \mathrm{C}, 59 \mathrm{C}$ and $87+73 \mathrm{C}$ mutants each can crosslink all 3 of the CCs in some combination, we expected that an $87+73+59 \mathrm{C}$ triple mutation would be able to crosslink all 3 of the CCs at once. However, after generating and exposing this triple PAN mutant to oxidizing conditions, we only observed $74 \pm 3 \%$ crosslinking (Fig. 4.7A), which is consistent with only 2 of the 3 CCs being crosslinked. But which of these two CCs are crosslinked? Upon further analysis of this triple mutant, we found that it functions in a similar fashion as WT PAN when uncrosslinked or crosslinked in both ATPase (Fig. 4.7B, S3; Table S4.1) and unfoldase (Fig. 4.7C) activity. The normal functioning of this triple mutant indicates that the $\mathrm{C} 1$ and $\mathrm{C} 2$ conformations most likely crosslink (87C-87C and 59C-59C) and the C3 conformation (87C-73C) does not, since if the C3 conformation crosslinked one would expect a substantial decrease in both ATPase and unfoldase (as observed in PAN 87+73C) (Fig. 4.6A-B). This result demonstrates that the $\mathrm{C} 2$ and $\mathrm{C} 3$ conformations cannot be simultaneously crosslinked, because only 2 of the 3 possible crosslinks could simultaneously form in this triple mutant. Based on this data we hypothesize that there are 2 conformational states of PAN, State \#1 where C1 \& C3 are crosslinkable (which $87+73 \mathrm{C}$ mutant crosslinking can capture), and State \#2 where C1 \& C2 are crosslinkable (which the 59C mutation can

capture) (Fig. 4.7D). State \#1 appears to exist in a resting state while state \#2 appears to have a slightly activated ATPase activity ( $25 \%$ above that of its reduced control) (Table S4.2). We also found that the triple mutation $(87+73+59 \mathrm{C})$, which could potentially crosslink either state 1 or state 2 , preferentially crosslinked state \#2 as it had 


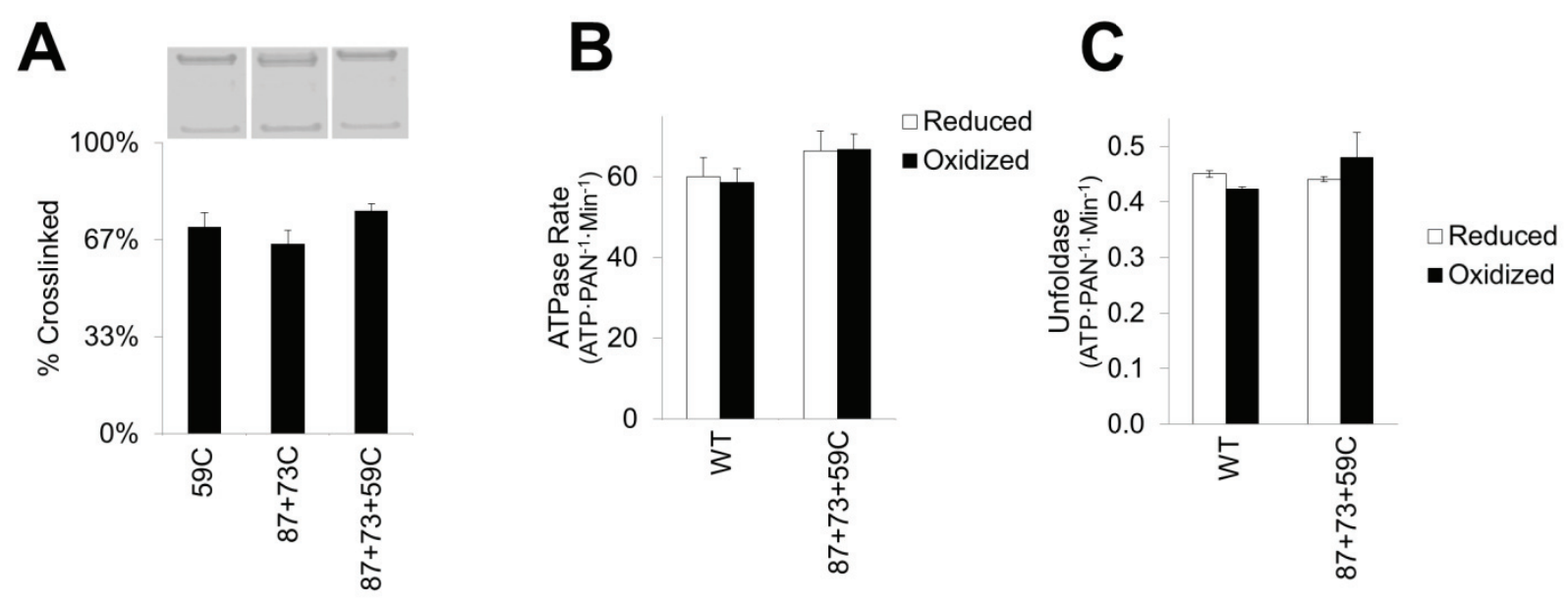

D
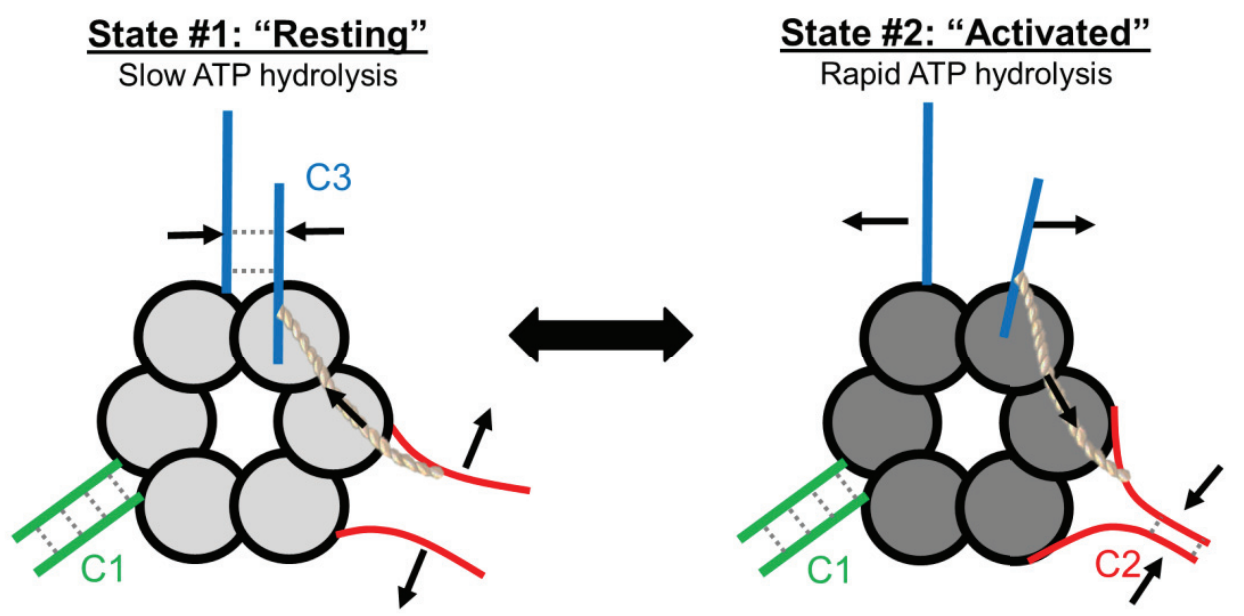

Figure 4.7: PAN Adopts 2 Distinct States: One that is Fully Active and Another that is Resting

A) The PAN triple mutant (PAN $87 C+73 C+59 C$ ) was subjected to oxidizing conditions and compared to PAN 59C and PAN 87C+73C. Crosslinking experiments were performed in triplicate and representative images are from a noncontiguous gel. ATPase Activity (B) and Unfoldase activity (C) of PAN $87 \mathrm{C}+73 \mathrm{C}+59 \mathrm{C}$ under reduced and oxidized conditions was determined and compared to WT. Bar graph values are means \pm standard deviations of 3 independent experiments $(n=3)$. D) Model representing the conformational asymmetry of PAN's CC domains, and how conformational switching in the $\mathrm{CC}$ domains regulates its activity. In State \#1, PAN is in a "resting" state with slowed ATP hydrolysis and substrate processing, and C1 (green) and C3 (blue) CCs are crosslinkable. However, due to allosteric restrictions (indicated by the rope linking the blue and red CCs), C2 (red) is not crosslinkable in state \#1. In State \#2, PAN is fully active with rapid ATP hydrolysis and substrate processing, and the C1 (green) and C2 (red) CCs are crosslinkable, while the C3 conformation (blue) is not crosslinkable. 
a more similar functional profile to state \#2 (59C) than state \#1 (87+73C) (Table S4.1, Fig. 4.6A-B, 4.7A-C, S4.3). This indicates that PAN crosslinks more easily in State \#2, which prevents switching to state \#1. However, if state \#2 is not stabilized via crosslinking then state \#1 can be captured with the $87+73 \mathrm{C}$ mutant. This regulation of state changes in the ATPase domains (regulated by CC conformation) seems to be a theme in the proteasomal ATPases and these results for PAN have parallels with state changes in the eukaryotic 26S proteasome (discussed below). 


\section{Discussion}

This study uncovered several surprising properties of the structure and function of the PAN proteasomal ATPase: 1) that the CC domains in PAN are not conformationally symmetric, 2) the global conformation of the CCs does not appear to cycle around the ring, and 3) local changes in two of the $\mathrm{CC}$ conformations (C2 and $\mathrm{C} 3$ ) can regulate PAN's activity. Previous biochemical studies have suggested that PAN adopts asymmetrical conformations $48,51,52$, but the present study provides the first direct structural evidence of this, at least for the CC domains. This finding is critical, because up until this point it has been assumed based on crystal structures of PAN's N-terminus that it is symmetric. Interestingly, this CC conformational asymmetry in the homohexamer PAN also extends to the eukaryotic 26S proteasomal ATPases (Rpt1-6). Although the CC conformational asymmetries in Rpt1-6 have not been previously discussed, it is clear from the cryo-EM structures that the CC domains are also asymmetric, though this is less surprising since Rpt1-6 is a heterohexamer. In fact, one structure of the 19S ATPase's CC domains (PDB: 4CR4) shows its Rpt4/5 CC in a mostly "zipped" conformation (similar to the 'C1' conformation found in PAN), while the Rpt6/3 CC is partially unzipped (similar to the 'C2' conformation found in PAN), and the Rpt1/2 CC is 2-heptads out-of-register (similar to the 'C3' conformation found in PAN) (Fig S4.6) $)^{5}$. We measured the $\beta$-carbon distances and angles between each of the hydrophobic residues in the Rpt CCs and found that if similar cysteine mutations were introduced in this structure, the Rpt 4/5 (C1) and Rpt 6/3 (C2) conformations would crosslink but Rpt 1/2 (C3) would not, consistent with the results we found here for PAN (e.g. 59C). Therefore, our findings with disulfide crosslinking of PAN's CC domains are 
fairly consistent with the conformation of CC domains in the $26 \mathrm{~S}$. This suggests that the $\mathrm{CC}$ conformational asymmetry is inherent to the proteasomal ATPase rings and existed prior to the development of eukaryotes. This suggests that the AAA+ ATPase domains of PAN and the 19S likely hexamerize in a similar conformational fashion (e.g. a lockwasher), which provides a structural explanation of previous observations that the 19S and PAN share similar nucleotide binding, hydrolysis, and substrate processing characteristics where both PAN and the 19S ATPases 1) bind to 2 ATPs ${ }^{48}$ 2) bind ATPs on adjacent subunits ${ }^{52}, 3$ ) have 2 ADP (or low affinity) sites and 2 empty sites ${ }^{48}$, and 4) are similarly highly processive when engaged with substrate ${ }^{51}$. These results therefore continue to illustrate the strength of PAN as a model system for the 195 to understand at least the fundamental functions of these machines. However, the $19 \mathrm{~S}$ also has many associated non-ATPase subunits (unlike PAN) that form many contacts with the CC domains that almost certainly further influence heterogeneity of the CC conformations, (for example, the "kink" in the CC that has been observed in the Rpt1/2 CC of some recent $19 \mathrm{~S}$ structures) ${ }^{8,13,14}$.

The disulfide crosslinking methods we employ in this manuscript can precisely show whether specific residues come within $3.4-4.6 \AA$ of one another at the appropriate angle ${ }^{47}$. Therefore, any residues that form a disulfide crosslink can unambiguously be determined to have (at least momentarily) passed through a $1.2 \AA$ space, whereas the available cryo-EM structures have a more limited resolution-especially in the CC domains. For example, as of the writing of this manuscript, the best cryo-EM average resolution of the $26 \mathrm{~S}$ proteasome is $3.5 \AA$ ( $5 \mathrm{GJR})$, however, upon analysis of the 
structure's B-factors, we estimate that most portions of the Rpt CCs have resolutions of $\sim 10-15 \AA^{13}$. Therefore, general trends in the conformations these CC structures may adopt can be estimated by the available cryo-EM structures, but the disulfide crosslinking approach we have designed provides a much more precise tool for determining the proximity of specific residues, and thus CC conformational states, in PAN. Using this disulfide crosslinking approach, we determined that PAN can adopt two different states: One with high activity (stabilized by crosslinking the C2 conformation) and one with lower activity (stabilized by crosslinking the C3 conformation (Fig. 4.7D). Interestingly, these two states are mutually exclusive (i.e. they "switch") from one another. This indicates that each $\mathrm{CC}$ must also have a secondary conformation that we could not capture via disulfide crosslinking here and so we can only speculate that these secondary states are perhaps unstructured.

The majority of ATPase ring complexes, including the proteasomal ATPases, are expected to hydrolyze ATP in a cyclical fashion. Based on this model we expected that the CC domains would switch between their 3 different conformations during a full $360^{\circ}$ ATP hydrolysis cycle. Indeed, the C2 and C3 conformations appear to fluctuate during ATP hydrolysis; however, crosslinking the $\mathrm{C} 1$ conformation of PAN (87C mutant) had absolutely no effect on ATPase activity or protein unfolding rates. This indicates that the C1 conformation can be static and unchanging during normal function. This was somewhat of a surprise since it's assumed that ATP is hydrolyzed in all subunits around the ring. How can we interpret sequential ordered ATP hydrolase around the ring in light of this result? We envision 2 possibilities: 1) conformational changes in the ATPase 
subunits that produce work due to ATP-binding and hydrolysis are not directly and tightly correlated to the CC conformations, or 2) ATP hydrolysis only occurs in four of the six ATPase subunits (i.e. those linked to the C2 and C3 conformations, which are more dynamic). We favor possibility \#1 but cannot rule out \#2. ATP hydrolysis in a subset of subunits in the $26 \mathrm{~S}$ has been previously proposed elsewhere (Beckwith et al. 2013). In case \#1, how does regulation of ATPase activity by substrate binding fit in? The most stable state for any parallel CC domain is zipped and in register. The fact that $\mathrm{C} 2$ and $\mathrm{C} 3$ are not in the $\mathrm{C} 1$ conformation indicates there are allosteric conformational restraints within the hexameric ring that only permit one $\mathrm{C} 1$ conformation, causing the others to adopt alternative states (i.e. C2 and C3). Thus, the C1 conformation breaks PAN's hexameric symmetry and "locks" it into a conformationally asymmetric state. Interestingly, the $\mathrm{C} 1$ conformation relies on $\mathrm{M} 87$, and when it is mutated to an alanine we observe an increase in PAN's basal ATPase activity (Fig. 4.6C). Based on this we hypothesize that the C1 conformation may tend to pause cycles of ATP hydrolysis at this pair of subunits during normal PAN function. In this way the $\mathrm{C} 1$ conformation may add a slight energy barrier to overcome before committing to another cycle of ATP hydrolysis, and the M87 $\rightarrow$ A mutation reduces this energy barrier by slightly and locally destabilizing the proximal end of the $\mathrm{C} 1 \mathrm{CC}$. An analogy would be a speed bump on a road, which is not intended to stop the flow of cars, but to slow them. Similarly, substrate binding to the CC domains could lower this "energy barrier" and promote subsequent and more frequent rounds of ATP-hydrolysis (i.e. accelerate ATPhydrolysis) for more rapid unfolding and degradation. It appears that PAN activation by M87A mutation or by substrate binding converge on a single "activation" mechanism, 
because both activate PAN, but when combined together their effect is not additive (activation by GFP-ssrA = activation by GFP-ssrA + activation by M87A; Fig. 4.6C). We postulate that this shared mechanism is via conformational alteration of PAN's CC domains, perhaps by shifting the population of PANs with C2 versus C3 conformations. Prior analysis of the eukaryotic $26 \mathrm{~S}$ proteasome via cryo-EM, has shown that the $26 \mathrm{~S}$ proteasome can be found in low energy or "resting" states and in "activated" (or substrate-bound like) states ${ }^{1}$. It's feasible that aspects of these CC conformational arrangements regulating ATPase activity could be conserved between archaea and eukaryotes. Further work will be required to solidify and differentiate between these models.

It is known that the 19S ATPases (like PAN) are activated by substrate binding to ubiquitin receptors, so we propose that the CC domains in the 19S ATPases may adopt similar conformations to transmit allosteric signals to the AAA+ ATPase domains to increase ATP hydrolysis. Similarly, deubiquitinases associated with the CC domains (directly or indirectly) could allosterically regulate the ATPase subunits to modulate the timing of substrate engagement with deubiquitination ${ }^{7,53,54}$. Many post-translational modifications (PTMs) have also been observed in the 26S, some of which are activating and others that are inactivating ${ }^{17-28}$. Several of these PTMs have been observed in the $\mathrm{N}$-terminal domains of the proteasomal ATPases. It is therefore likely that the conformational changes in the $\mathrm{CC}$ domains regulated by these post-translational modifications could also help to "switch" the proteasome between its activated and resting states. Thus, a fundamental mechanistic understanding of conformational 
changes in the proteasomal ATPase's CC domains will be essential to understanding the nuances of how the $26 \mathrm{~S}$ is regulated.

This study also overcomes a major limitation of PAN: its homomeric nature, which previously made it difficult to study its asymmetric properties like nucleotide binding. While others have circumvented this problem in distantly related homomeric ATP-dependent proteases (e.g. ClpX) by cleaving off the $\mathrm{N}$-domains to generate $\mathrm{C}$-to- $\mathrm{N}$ linked pseudohexamers to probe ring asymmetries, we determined that the $\mathrm{N}$-domains of PAN have a crucial role in regulating the activity of the ATPase domains, so a parallel approach in PAN would be functionally deleterious. These crosslinkable PAN mutants now facilitate and open the door for future studies of structural asymmetries in PAN in native states, which serves as a starting point to further elucidate the mechanism of how the proteasomal ATPase uses the energy from ATP to drive protein degradation. 


\section{Methods}

\section{Materials, Plasmids, and Protein Purification}

PAN, GFPssrA, and "T20S" (20S from Thermoplasma acidophilum) were prepared as described ${ }^{55,56}$. Saturating reducing reagent (1mM DTT) was maintained at each purification step, then proteins were buffer exchanged (either by dialysis or PD-10 columns), snap frozen in liquid nitrogen, and stored at $-80^{\circ}$ until use. Expression vectors for the PAN coiled-coil mutants in PRSETA were generated by site-directed mutagenesis (QuikChange II Mutagenesis Kit) and were confirmed by sequencing (Sequetech). The purest available forms of ATP, and ATPYS were purchased from Sigma and stored at $-80^{\circ} \mathrm{C}$ until use.

\section{Preparation of Mini-Spin G50 Columns}

24 hours prior to oxidation experiments, G50 (Illustra Sephadex G-50 Fine DNA Grade, GE) was preswollen in reaction buffer (50 mM Tris $\mathrm{pH} 7.5+5 \% \mathrm{v} / \mathrm{v}$ glycerol) at room temperature ( $1 \mathrm{~g}$ of G50 per $10 \mathrm{ml}$ of reaction buffer). The day of a disulfide crosslinking experiment, $900 \mu \mathrm{l}$ of G50 slurry was added to Pierce $0.8 \mathrm{ml}$ Centrifuge Columns (Thermo Scientific). Mini-spin columns were equilibrated 3 times in fresh reaction buffer: $500 \mu \mathrm{l}$ of reaction buffer was added to the column followed by centrifugation at $1500 \times \mathrm{G}$ for 60 seconds, this was repeated two more times. On the last equilibration step fresh buffer was added to column and the column was capped. Immediately prior to use, columns were uncapped and spun at 1500 x G for 60 seconds. The size of the columns were measured (distance from the top of the tube to the center of the column), and any columns that were not within $1 / 2 \mathrm{~mm}$ of one another were discarded as was any residual 
G50 that had not properly packed into the column. The center of a typical column (with an initial $900 \mu$ l slurry) was $450-500 \mu \mathrm{l}$ in volume, and was 6-7 $\mathrm{mm}$ below the top rim of the tube (1-2 $\mathrm{mm}$ below the threading marks).

\section{PAN Coiled-Coil Mutant Crosslinking and Analysis}

PAN CC mutants were stored at $-80^{\circ}$ and were thawed on ice immediately prior to use. In every oxidation reaction, a reduced CC mutant control (1 mM DTT) and an oxidized WT control were run in tandem. All oxidation reactions were done in $50 \mathrm{mM}$ Tris $\mathrm{pH} 7.5+5 \%$ glycerol $(\mathrm{v} / \mathrm{v})$, and were done in the absence of nucleotides, unless otherwise noted. The oxidizing reagent, tetrathionate, (Sodium tetrathionate dehydrate $\geq$ $98 \%$, Sigma) was used to oxidize the cysteines to allow for disulfide crosslink formation. Saturating tetrathionate concentration was determined for each CC mutant via dose response, and this level of oxidizing reagent was used for crosslinking assays unless otherwise indicated. In general, $1 \mathrm{mM}$ of tetrathionate for 1-2 hours was sufficient to induce saturable crosslinking in the PAN CC mutants. Some PAN mutants have a tendency to form dodecamers (especially those carrying double cysteine mutations). Therefore, all PAN CC mutants were diluted to $\leq 0.25 \mathrm{mg} / \mathrm{ml}$ during disulfide crosslinking, which greatly minimized dodecamerization. Trace amounts of tetrathionate present in the samples upon addition to SDS sample buffer caused non-specific disulfide crosslinks to form since SDS denatures PAN. Therefore, after oxidation but prior to SDS-PAGE analysis each PAN was carefully desalted on G50 mini-spin columns. 20ul of each sample was added to mini spin G50 columns which were prepared as described above. Following desalting the concentrations of each sample 
was recalculated via Bradford assay immediately after desalting. $\sim 50 \%$ of PAN was recovered after each desalting column, to assure that tetrathionate was completely removed. Crosslinking of every sample was confirmed via SDS-PAGE analysis prior to use. $3 \mu \mathrm{g}$ per well was used for SDS-PAGE analysis. Following SDS-PAGE, gels were rinsed 3 times with double deionized water, stained with Bio-Safe Coomassie G-250 Stain (Biorad) for 1 hour, rinsed in $100 \mathrm{ml}$ of double deionized water for 1 hour, and then $20 \mathrm{ml}$ of $20 \% \mathrm{NaCl}(\mathrm{w} / \mathrm{v})$ was added for at least 1 hour. Gels were then imaged on a Syngene GBox Imager. We found using this strict staining/rinsing method that the densitometry signal remained linear between $0.15-5 \mu \mathrm{g}$ PAN (Fig. S4.7), which allowed for accurate calculation of the relative amounts of PAN within this range. Disulfide crosslink ratio was determined by calculating the pixel intensity of monomers vs. dimers in ImageJ after subtracting control densities. Data are means of at least 3 independent experiments $(n \geq 3) \pm$ standard deviations.

\section{Partial Proteolysis of PAN}

$0.4 \mathrm{mg} / \mathrm{ml}$ of PAN was mixed with increasing amounts of Trypsin (0-2 $\mu \mathrm{g}$ per $40 \mu \mathrm{l} r \mathrm{nn})$, incubated for 1 hour at room temperature in reaction buffer $(50 \mathrm{mM}$ Tris $\mathrm{pH} 7.5,5 \% \mathrm{v} / \mathrm{v}$ glycerol), and reactions were quenched with manufacturer recommendation amounts (1:100) of Halt Protease Inhibitor Cocktail (Thermo Scientific). Samples were checked for $\mathrm{CC}-\mathrm{OB}$ domain fragment via Native-PAGE, and samples containing $\mathrm{CC}-\mathrm{OB}$ domain fragment were pooled and injected onto a size exclusion column (Superose $12 \mathrm{HR}$ 10/30, GE). Immediately after elution 1:100 Halt Protease Inhibitor Cocktail (Thermo Scientific) was added to each fraction. Peaks were analyzed via SDS-PAGE and 
Native-PAGE and Fraction 1 (containing near-full-length PAN) and Fraction 3 (containing $\mathrm{CC}-\mathrm{OB}$ fragment) were pooled (Fig. 4.3B). Fractions 1 and 3 were incubated with either $1 \mathrm{mM}$ DTT (reduced sample) or $1 \mathrm{mM}$ Tetrathionate (oxidized sample) for 1 hour at room temperature. After desalting (as described above), samples were run on SDS-PAGE and visualized via silver stain (Pierce Silver Stain Kit, Thermo Scientific). Representative Images from 4 independent experiments are shown.

\section{PAN-M87C crosslinking Timecourse}

$1 \mathrm{mg} / \mathrm{ml}$ PAN-M87C was incubated overnight @ $4^{\circ}$ with reducing agent (DTT). Reactions were carried out at $\left(-17^{\circ}\right)$, which was achieved using $11 \% \mathrm{NaCl}$ in ice $(\mathrm{w} / \mathrm{w})$. This temperature was maintained for 6 hours, after which the temperature had risen to $\left(-15^{\circ}\right)$. Samples contained $50 \%$ glycerol to prevent freezing ${ }^{49}$. Immediately prior to an

experiment, samples were desalted in G50 (see "PAN Coiled-Coil Mutant Crosslinking and Analysis" section), and diluted to $0.25 \mathrm{mg} / \mathrm{ml}$ in $50 \mathrm{mM}$ Tris pH $7.5+50 \%$ glycerol. $1 \mathrm{mM}$ tetrathionate was added to start the oxidation reaction, and at each timepoint a sample was immediately desalted and added to a $1 \times$ SDS sample buffer for analysis via SDS-PAGE.

\section{ATPase, GFP Unfolding, and 20S Gate Opening Assays}

All experiments were performed at $37^{\circ}$ with absorbance or fluorescence measured in a Biotek 96-well plate reader. Data are means of at least 3 independent experiments $(n \geq 3) \pm$ standard deviations. ATP hydrolysis was measured by using an $\mathrm{NADH}$-coupled ATP regenerating system (50 mM Tris $\mathrm{pH}$ 7.5, 5\% glycerol (v/v), $20 \mathrm{mM}$ 
$\mathrm{MgCl}_{2}, 2 \mathrm{U} / \mu \mathrm{l}$ pyruvate kinase, $2 \mathrm{U} / \mu \mathrm{l}$ lactate dehydrogenase, $3 \mathrm{mM}$ phosphoenolpyruvate, and $0.2 \mathrm{mg} / \mathrm{ml} \mathrm{NADH}$ ) by reading the loss of NADH absorbance at 340nm every 20 s. 2 mM ATP was used unless otherwise indicated (e.g. ATP doseresponse curves).

For the unfolding experiments, reaction buffer (50 mM Tris $\mathrm{pH} 7.5,5 \%$ glycerol $(\mathrm{v} / \mathrm{v}))$ was incubated with $20 \mathrm{mM} \mathrm{MgCl}$, $50 \mathrm{nM}$ PAN, $400 \mathrm{nM}$ T20S, and $0.2 \mathrm{nM}$ GFPssrA and 2 mM ATP. GFP fluorescence loss (ex/em: 485/510) was measured every $20 \mathrm{~s}$ in a Biotek 96 well-plate reader at $37^{\circ}$ to obtain unfolding rates. Data are means of at least 3 independent experiments $(n \geq 3) \pm$ standard deviations.

Gate opening was measured in the reaction buffer with the archaeal T20S (3 $n M)$, PAN mutants $(200 \mathrm{nM})$, and $\mathrm{MgCl}_{2}(20 \mathrm{mM})$ using the internally quenched fluorogenic peptide substrate (LFP) ${ }^{56}$. LFP was dissolved in dimethylsulphoxide and used at a final concentration of $10 \mu \mathrm{M}$ in the presence or absence of $10 \mu \mathrm{M}$ ATPYS. LFP contains a fluorescent reporter (MCA) at the $\mathrm{N}$-terminus and a quenching group (DNP) at the C-terminus. Upon cleavage of the peptide by the $20 \mathrm{~S}$ proteasome, MCA is released and an increase in fluorescence can be observed at ex/em: 325/393. Rate of fluorescence increase (ex: 325 em: 393) was measured every $20 \mathrm{~s}$ in a biotek 96 well plate reader to determine the rate of $20 \mathrm{~S}$ activation (gate opening) by PAN.

\section{S Structure Analysis and PAN Mass Spectrometry analysis}

$19 S$ cryo-EM structures (e.g. PDB: 4CR4) were analyzed in Pymol. $\beta$-carbon distances were measured using the "Measurement" tool. 


\section{Mass Spectrometry Analysis}

Uncrosslinked and crosslinked PAN-M87C samples were trypsinized and sent for analysis by the "Scripps Center for Metabolomics" in La Jolla, CA. Crosslinked samples were analyzed in a non-reducing environment. 
Supplemental Information 


\section{Table S4.1: Summary of ATP Hydrolysis Kinetics of PAN Variants Under Oxidizing and Reducing Conditions}

\begin{tabular}{|c|c|c|c|c|c|c|c|c|}
\hline & \multicolumn{4}{|c|}{ Reduced } & \multicolumn{4}{|c|}{ Oxidized } \\
\hline & $\underset{\text { (ATPPAN-1-1.min-1) }}{\operatorname{Vmax}}$ & $\underset{[A T P] \mu M}{\mathrm{Km}}$ & Hill & $\begin{array}{c}\text { CC } \\
\text { Crosslink }\end{array}$ & $\underset{\left(A T P \cdot P A N^{-1} \cdot m_{i n-1)}\right.}{\operatorname{Vmax}}$ & $\underset{[A T P] \mu M}{\mathrm{Km}}$ & Hill & $\begin{array}{c}\text { CC } \\
\text { Crosslink }\end{array}$ \\
\hline WT PAN & $61.2 \pm 2$ & $507 \pm 37$ & $1.8 \pm 0.2$ & None & $55.9 \pm 2.9$ & $457 \pm 56$ & $1.7 \pm 0.3$ & None \\
\hline M87C & $53.8 \pm 1.9$ & $413 \pm 34$ & $1.8 \pm 0.2$ & None & $52.7 \pm 2.3$ & $497 \pm 48$ & $1.8 \pm 0.1$ & C1 \\
\hline $59 C$ & $48.1 \pm 1.4$ & $436 \pm 29$ & $1.8 \pm 0.2$ & None & $56 \pm 2$ & $443 \pm 36$ & $1.8 \pm 0.2$ & $\mathrm{C} 1+\mathrm{C} 2$ \\
\hline $87+73 C$ & $42.2 \pm 1$ & $556 \pm 29$ & $1.8 \pm 0.1$ & None & $\begin{array}{l}\text { could not } \\
\text { determine }\end{array}$ & $>3000$ & \begin{tabular}{|l|} 
could not \\
determine
\end{tabular} & $\mathrm{C} 1+\mathrm{C} 3$ \\
\hline $87+73+59 C$ & $51.3 \pm 2.6$ & $580 \pm 64$ & $1.8 \pm 0.3$ & None & $53 \pm 3$ & $637 \pm 83$ & $1.6 \pm 0.3$ & $\mathrm{C} 1+\mathrm{C} 2$ \\
\hline
\end{tabular}

Values are calculated from curves in Figure S3. Values are means \pm standard deviation of 3 or more independent experiments $(n \geq 3)$. $87+73 C$-oxidized values could not be determined because data did not fit a Michaelis-Menton Curve. 


\section{Table S4.2: Normalized ATP Hydrolysis of PAN Variants}

\begin{tabular}{|c|c|c|}
\hline & $\begin{array}{c}\text { Reduced } \\
\text { Vmax } \\
\text { (normalized) }\end{array}$ & $\begin{array}{c}\text { Oxidized } \\
\text { Vmax } \\
\text { (Normalized) }\end{array}$ \\
\hline WT PAN & $100 \pm 3.3 \%$ & $100 \pm 5.2 \%$ \\
\hline M87C & $100 \pm 3.5 \%$ & $107 \pm 4.7 \%$ \\
\hline $59 C$ & $100 \pm 2.9 \%$ & $128 \pm 4.6 \% * *$ \\
\hline $87+73 C$ & $100 \pm 2.4 \%$ & could not determine \\
\hline $87+73+59 C$ & $100 \pm 5.1 \%$ & $113 \pm 6.4 \%$ \\
\hline
\end{tabular}

Vmax values were calculated from curves in Figure S3 and values in Table S1. Vmax values were normalized to WT PAN controls and divided by the reduced form of the mutant. Values are means \pm standard deviation of 3 or more independent experiments $(n \geq 3)$. 87+73Coxidized values could not be determined because data did not fit a Michaelis-Menton Curve. ${ }^{* *}=p<0.001$ 

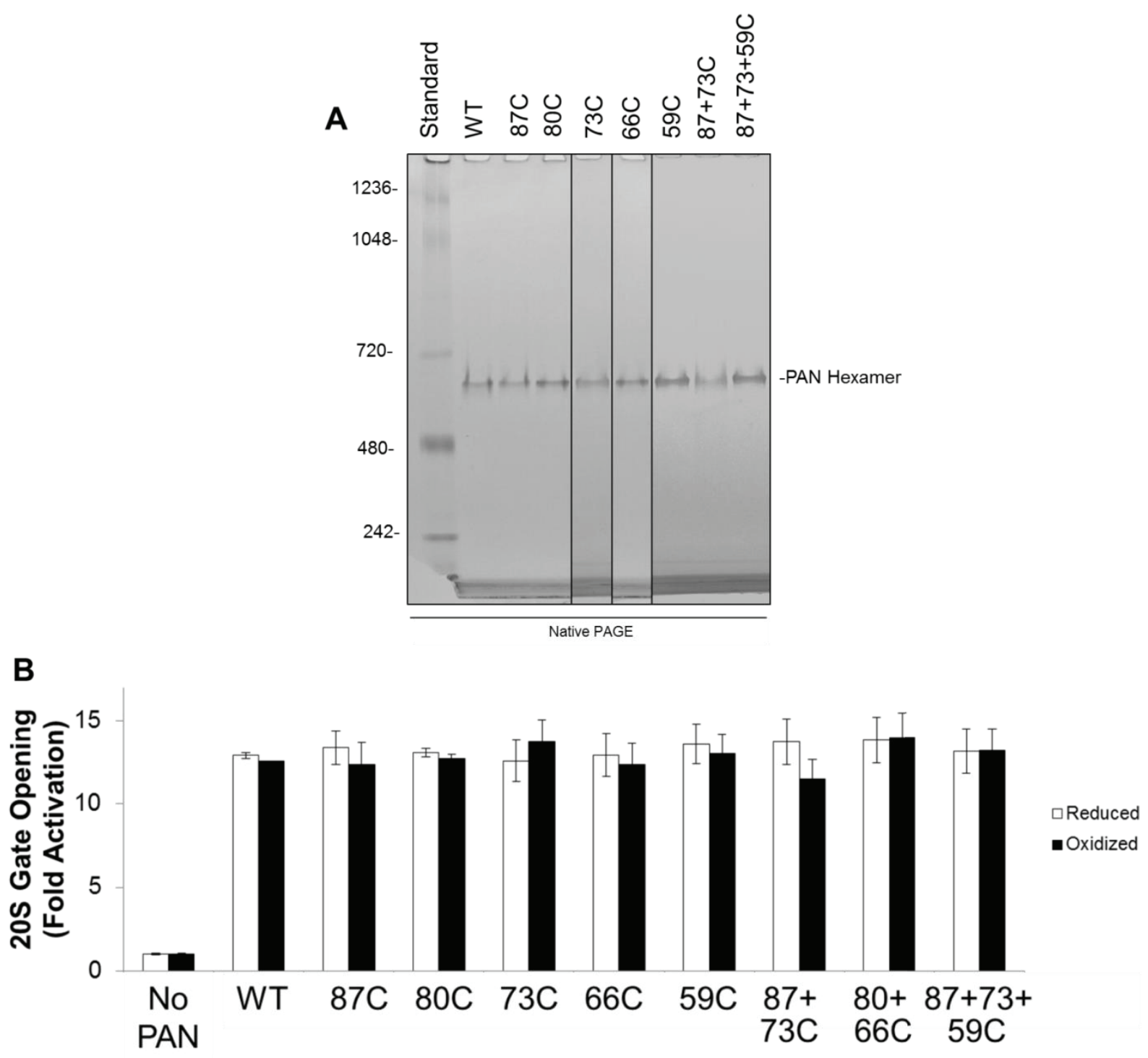

Figure S4.1: PAN Mutants Retain Global Quaternary Structure and T20S Gate Opening Capacity

A) $2 \mu \mathrm{g}$ of oxidized PAN variants were run on Native-PAGE and analyzed for hexamer formation. Experiments were performed in triplicate. Representative data is presented is from 3 noncontiguous gels. Note that PAN runs on Native gels at a higher molecular weight than expected, likely due to differences in tertiary/quaternary structure of PAN compared to the standards used. B) The stimulation of 20S activity (caused by PAN-induced 20S gate opening) was measured using saturating PAN and $2 \mathrm{uM}$ of a fluorescent reporter nonapeptide (LFP) with 10uM ATPYS and $20 \mathrm{mM} \mathrm{MgCl} 2$ (see methods for details). The rate of LFP hydrolysis was calculated and fold stimulation of the $20 \mathrm{~S}$ activity by PAN is shown. $20 \mathrm{~S}$ alone control is considered 1 -fold. 


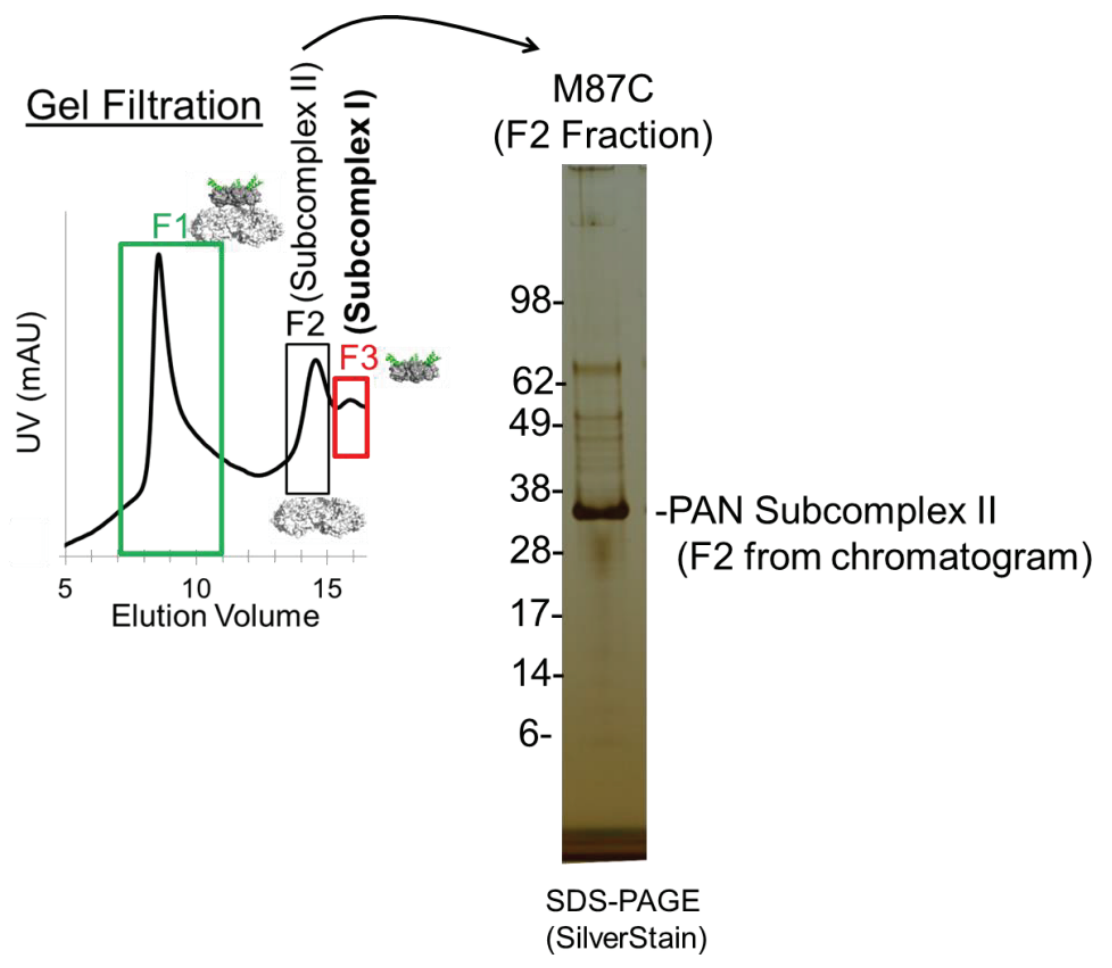

Figure S4.2: SDS-PAGE of the "F2" PAN-M87C fragment. Partial proteolysis fragments of PAN were loaded onto a GE Superose 12 size exclusion column. The F2 fragment ran as a 30$35 \mathrm{kDa}$ monomer, consistent with the monomeric size of PAN Subcomplex II (the AAA+ ATPase domain fragment). 
A

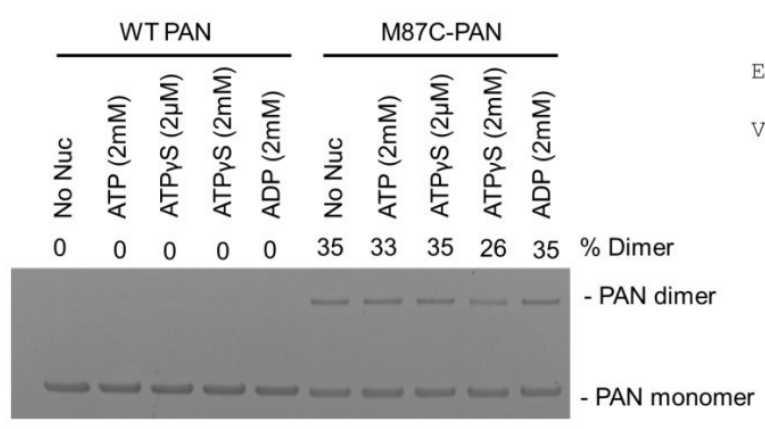

B $\quad \operatorname{PAN}(M$. jannaschii)

\begin{tabular}{|c|c|c|c|c|}
\hline 60 & 70 & 80 & 90 & 100 \\
\hline ELESRILKLE & LEKKELEREN & LQLAKENEIL & RRELDRCRVP & PLIVGTVVDK \\
\hline 110 & 120 & 130 & 140 & 150 \\
\hline GERKVVVKS & STGPSFLVNV & SHFVNPDDLA & PGKRVCLNQQ & TLTVVDVLPE \\
\hline
\end{tabular}

Corresponding peptide from mass spectrometry:

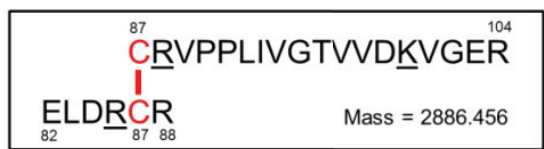

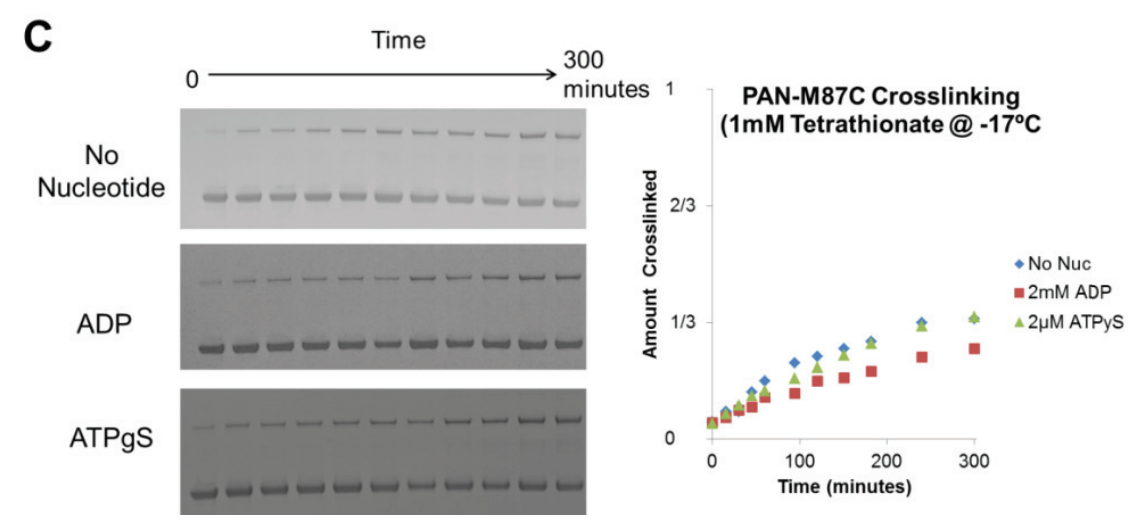

Figure S4.3: "Natural" levels of nucleotides have little effect on disulfide crosslinking of WT PAN or PAN-M87C. A) $0.25 \mathrm{mg} / \mathrm{ml}$ WT PAN or PAN-M87C was incubated for 1 hour @ room temperature with $1 \mathrm{mM}$ tetrathionate (and the indicated nucleotide $+10 \mathrm{mM} \mathrm{MgCl}$ ). Note that the level of crosslinking is approximately the same regardless of the nucleotide bound, with the exception of high levels of ATP $\mathrm{S}(2 \mathrm{mM})$, which has been previously shown to force PAN into an unnatural 4-nucleotide bound conformation (Smith et al. Mol Cell 2011). B) Mass spectrometry of PAN-M87C reveals a peptide with a mass corresponding to a dimer with a disulfide crosslink at residue 87. (top) Sequence of PAN (M. jannaschii) from residues 51-150. The $87^{\text {th }}$ residue mutated to cysteine is indicated in red. (bottom) A peptide of Mass $=2886.456$ Da was found in the oxidized sample, which corresponds to 2 fragments of PAN that had been crosslinked at residue M87C. The fragment from the first monomer was from 87-104, and the peptide from second PAN monomer from 82-88. Note that both of these crosslinked fragments have missed trypsin cleavage sites (underlined), which is expected to occur when a disulfide bond occludes trypsin's access to these cut sites. C) PAN-M87C crosslinking timecourse. $0.25 \mathrm{mg} / \mathrm{ml}$ PAN-M87C was reduced using $1 \mathrm{mM}$ dithiothreitol, desalted, and then incubated at ($17^{\circ} \mathrm{C}$ ) for $0-300$ minutes with $1 \mathrm{mM}$ tetrathionate and the indicated nucleotides. $-17^{\circ}$ temperatures were achieved with $11 \% \mathrm{NaCl}$ in Ice Water (w/w), and 50\% glycerol was used in samples to prevent freezing of samples (Lane Ind. Eng. Chem. 1925). Left panel is raw SDSPAGE data of these experiments (coomassie), right panel is quantification of SDS-PAGE. 


\section{A 'a' residue mutants}

$$
\begin{array}{r}
\text { Xlinked } \frac{84 \mathrm{C}}{13.5} \frac{77 \mathrm{C}}{10.4} \frac{70 \mathrm{C}}{4.8} \\
\text { (\%): } \pm 4.7 \pm 3.5
\end{array}
$$
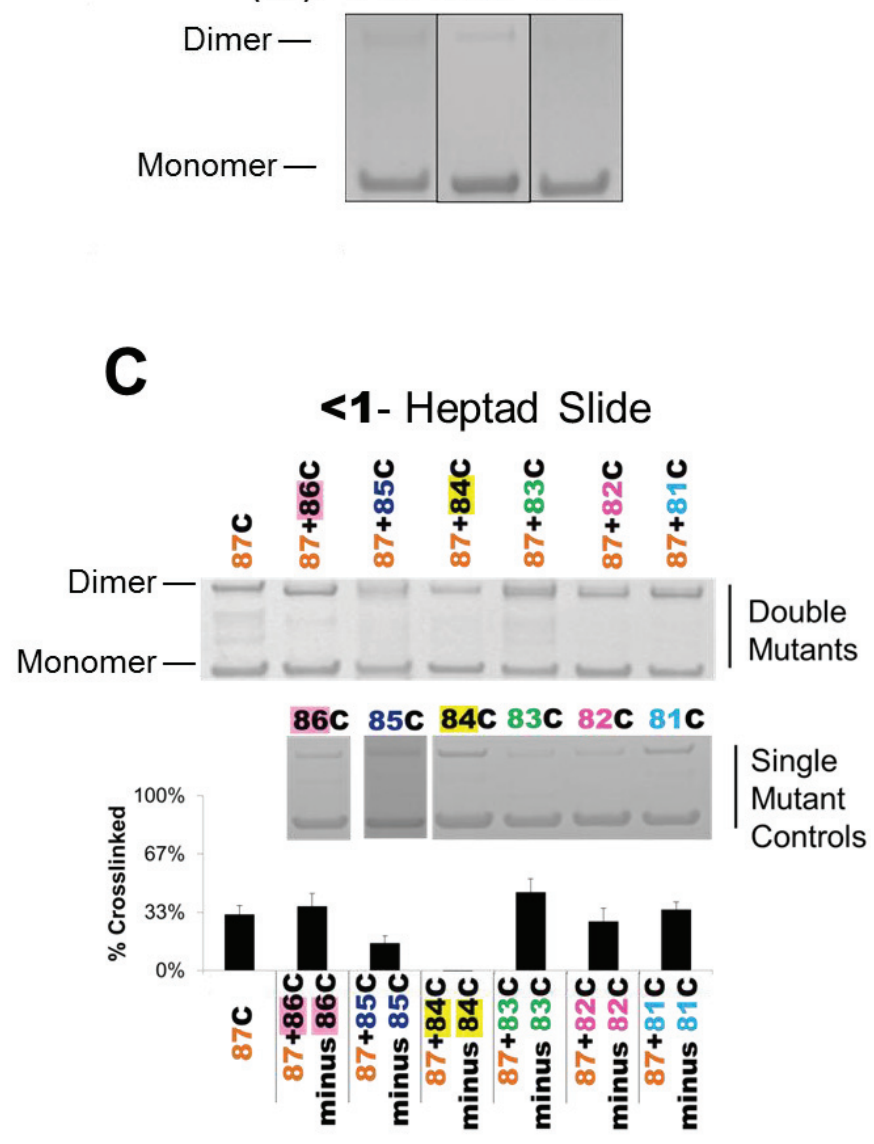

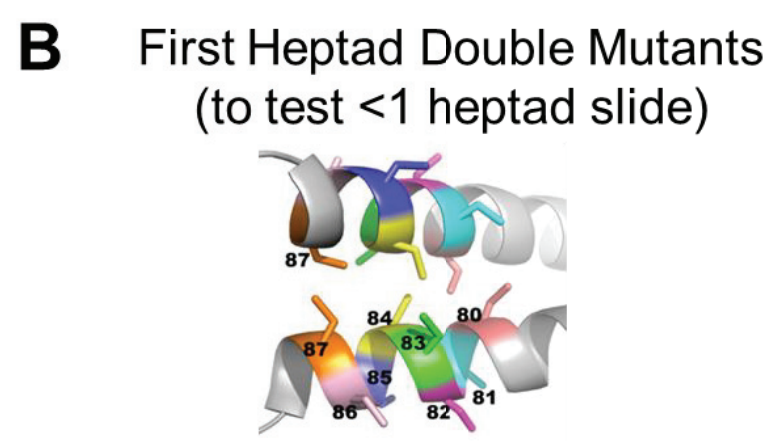

PDB: $3 \mathrm{H} 43$

Figure S4.4: PAN does not adopt a partial heptad slide.

A) Residues in the 'a' position of the heptad repeat were mutated to cysteines and subjected to crosslinking and SDS-PAGE analysis. B) Crosslinking strategy to test every possible registry shift at less than 1 heptad. Point mutations of each residue in the first heptad were generated either alone (single mutants) or with residue M87C (orange, double mutants). C) The indicated double mutants and their single mutant controls were subjected to oxidizing conditions $(1 \mathrm{mM}$ tetrathionate), desalted, and run on SDS-PAGE followed by coomassie staining. These mutants can crosslink in-register and in register slides of $<1$ heptad. This allowed the analysis of the level of crosslinking contributed by $\mathrm{C} 1$ (in-register $\mathrm{CC}$ ) plus the level of crosslinking contributed by an out-of-register CC, since single mutants can only crosslink in-register CCs. Note: some of the double mutants have less than $33 \%$ crosslinking after the background single mutant control is subtracted, likely due to destabilization of the CC. Bar graphs represent the amount of crosslinked PAN less the single mutants controls. 

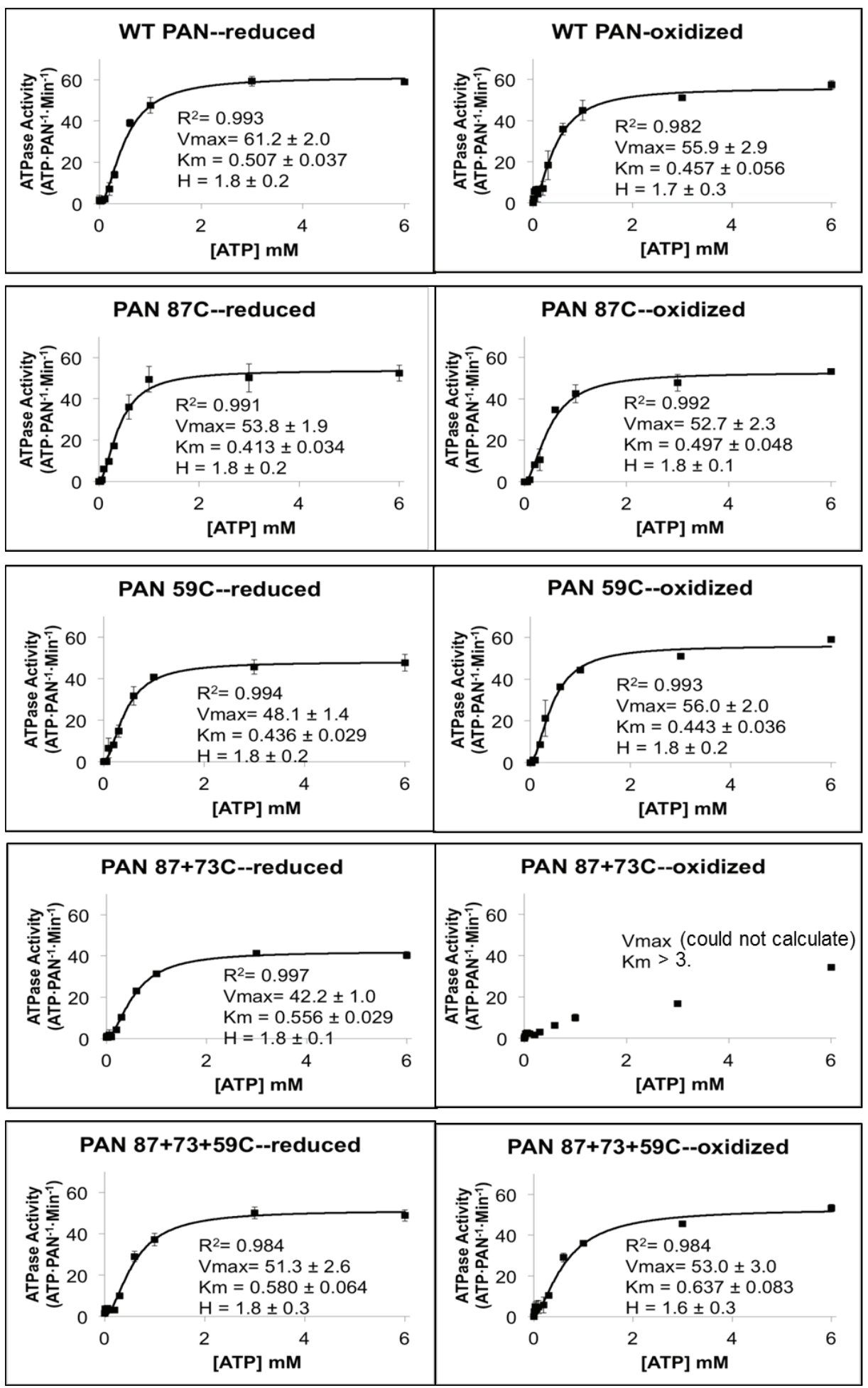

Figure S4.5: ATP hydrolysis kinetics of PAN mutants under oxidizing and reducing conditions

Increasing amounts of ATP $(0-6 \mathrm{mM})$ were added to PAN variant $(0.05 \mu \mathrm{M})$ under oxidizing and reducing conditions and ATPase activity was measured via NADH-coupled assay (see methods). Data was fit to 3 parameter Michaelis-Menten curve and $V \max , \mathrm{Km}$, and Hill coefficients were extracted and are shown. The PAN 87+73C-oxidized variant did not fit a Michaelis-Menten curve. 


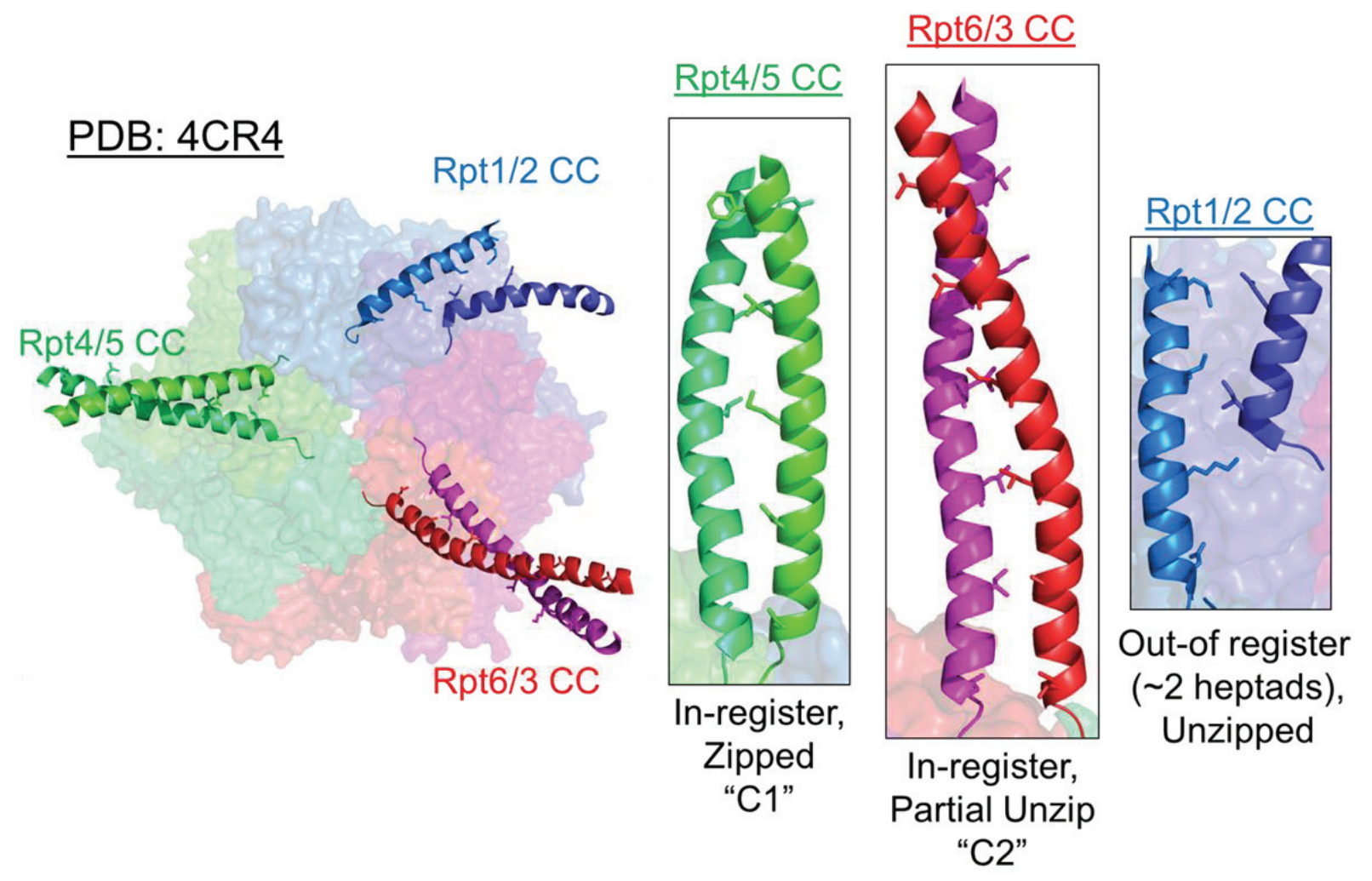

Figure S4.6: Asymmetric coiled-coil conformations are also observed in the 26S ATPases (PDB: 4CR4).

In the 4CR4 cryo-EM structure, the Rpt4/5 CC is in-register and mostly zipped (C1-like), the Rpt6/3 CC is in-register and partially unzipped (C2-like), and the Rpt1/2 CC is 2 heptads out-ofregister (C3-like), but appears unzipped, consistent with State \#2 ("activated"), where the C1 and C2 CC conformations are crosslinkable, but not the C3 CC conformation. 

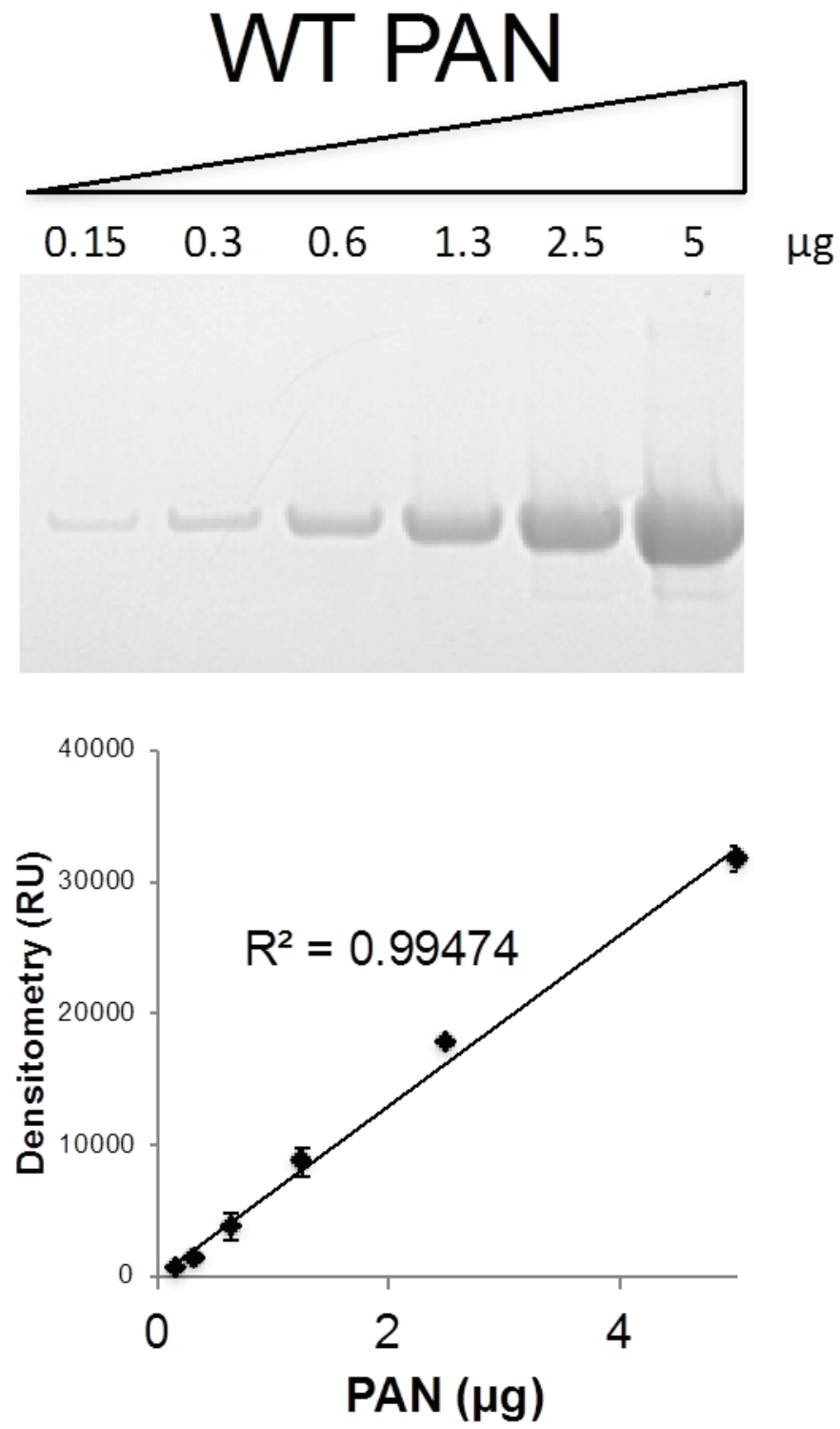

Figure S4.7: WT PAN dose response on SDS-PAGE. SDS-PAGE analysis of WT PAN dose response. Quantification (bottom) shows that densitometry increases linearly with PAN from 0.15$5 \mu \mathrm{g}\left(R^{2}=0.99474\right)$. Values are means \pm standard deviations of 3 independent experiments $(n=3)$. 


\section{References}

1. Matyskiela, M. E., Lander, G. C. \& Martin, A. Conformational Switching of the 26S Proteasome Enables Substrate Degradation. Nat. Struct. Mol. Biol. 20, 781-8 (2013).

2. Sledz, P. et al. Structure of the 26S Proteasome with ATP-yS Bound Provides Insights into the Mechanism of Nucleotide-Dependent Substrate Translocation. Proc. Natl. Acad. Sci. U. S. A. 110, 7264-9 (2013).

3. Ramnath, G. et al. Crystal structure of the proteasomal deubiquitylation module Rpn8-Rpn11. Proc. Natl. Acad. Sci. 111, 2984-2989 (2014).

4. Rosenzweig, R., Bronner, V., Zhang, D., Fushman, D. \& Glickman, M. H. Rpn1 and Rpn2 Coordinate Ubiquitin Processing Factors at Proteasome. J. Biol. Chem. 287, 14659-14671 (2012).

5. Unverdorben, P. et al. Deep Classification of a Large Cryo-EM Dataset Defines the Conformational Landscape of the 26S Proteasome. Proc. Natl. Acad. Sci. 111, 5544-5549 (2014).

6. Asano, S. et al. A molecular census of 26 Proteasomes in Intact Neurons. Science (80-. ). 347, 439-443 (2015).

7. Aufderheide, A. et al. Structural Characterization of the Interaction of Ubp6 with the 26S Proteasome. Proc. Natl. Acad. Sci. 112, 8626-8631 (2015).

8. Schweitzer, A. et al. Structure of the Human 26S Proteasome at a Resolution of 3.9 A. Proc. Natl. Acad. Sci. U. S. A. 113, 7816-21 (2016).

9. Chen, S. et al. Structural Basis for Dynamic Regulation of the Human 26S Proteasome. Proceedings of the National Academy of Sciences 113, (2016).

10. Wehmer, M. et al. Structural Insights into the Functional Cycle of the ATPase Module of the 26S Proteasome. Proc. Natl. Acad. Sci. 114, 1305-1310 (2017).

11. Ding, Z. et al. High-resolution cryo-EM structure of the proteasome in complex with ADP-AIFx. Cell Res. 1-13 (2017). doi:10.1038/cr.2017.12

12. Wang, X. et al. Molecular Details Underlying Dynamic Structures and Regulation of the Human 26S Proteasome. Mol. Cell. proteomics 16, 840-854 (2017).

13. Huang, X., Luan, B., Wu, J. \& Shi, Y. An atomic structure of the human $26 \mathrm{~S}$ proteasome. Nat. Struct. Mol. Biol. 18, 1-10 (2016).

14. Luan, B. et al. Structure of an Endogenous Yeast 26S Proteasome Reveals Two 
Major Conformational States. Proc. Natl. Acad. Sci. 113, 2642-7 (2016).

15. Inobe, T. \& Genmei, R. N-Terminal Coiled-Coil Structure of ATPase Subunits of 26S Proteasome Is Crucial for Proteasome Function. PLoS One 10, e0134056 (2015).

16. Inobe, T. \& Genmei, R. Inhibition of the 26S Proteasome by Peptide Mimics of the Coiled-Coil Region of its ATPase Subunits. Biochem. Biophys. Res. Commun. 468, 143-150 (2015).

17. Lokireddy, S., Kukushkin, N. V. \& Goldberg, A. L. cAMP-Induced Phosphorylation of 26S Proteasomes on Rpn6/PSMD11 Enhances their Activity and the Degradation of Misfolded Proteins. Proc. Natl. Acad. Sci. 112, E71767185 (2015).

18. Myeku, N. et al. Tau-driven 26S Proteasome Impairment and Cognitive Dysfunction can be Prevented early in Disease by Activating cAMP-PKA Signaling. Nat. Med. 22, 46-53 (2016).

19. Zhang, F. et al. Proteasome Function is Regulated by Cyclic AMP-Dependent Protein Kinase through Phosphorylation of Rpt6. J. Biol. Chem. 282, 22460-22471 (2007).

20. Satoh, K., Sasajima, H., Nyoumura, K. I., Yokosawa, H. \& Sawada, H. Assembly of the 26S Proteasome is Regulated by Phosphorylation of the p45/Rpt6 ATPase Subunit. Biochemistry 40, 314-319 (2001).

21. Maupin-Furlow, J. A., Humbard, M. A., Reuter, C. J., Zuobi-Hasona, K. \& Zhou, G. Phosphorylation and Methylation of Proteasomal Proteins of the Haloarcheon Haloferax volcanii. Archaea 2010, 1-10 (2010).

22. Gomes, A. V. et al. Mapping the Murine Cardiac 26S Proteasome Complexes. Circ. Res. 99, 362-371 (2006).

23. Rivett, A. J., Bose, S., Brooks, P. \& Broadfoot, K. I. Regulation of Proteasome Complexes by $\mathrm{y}$-Interferon and Phosphorylation. Biochimie 83, 363-366 (2001).

24. Zhang, F. et al. O-GlcNAc Modification is an Endogenous Inhibitor of the Proteasome. Cell 115, 715-725 (2003).

25. Mason, G. G. F., Murray, R. Z., Pappin, D. \& Rivett, A. J. Phosphorylation of ATPase Subunits of the 26S proteasome. FEBS Lett. 430, 269-274 (1998).

26. Kikuchi, J. et al. Co- and Post-translational Modifications of the 26S Proteasome in Yeast. Proteomics 10, 2769-2779 (2010).

27. Hirano, H., Kimura, Y. \& Kimura, A. Biological Significance of Co- and Post158 
translational Modifications of the Yeast 26S Proteasome. J. Proteomics 134, 37-46 (2016).

28. Collins, G. A. \& Goldberg, A. L. The Logic of the 26 S Proteasome. Cell 169, 792 806 (2017).

29. VerPlank, J. J. S. \& Goldberg, A. L. Regulating Protein Breakdown through Proteasome Phosphorylation. Biochem. J. 474, 3355-3371 (2017).

30. Wilson, H. L., Ou, M. S., Aldrich, H. C. \& Maupin-Furlow, J. Biochemical and Physical Properties of the Methanococcus jannaschii 20S Proteasome and PAN, a Homolog of the ATPase (Rpt) Subunits of the Eucaryal 26S Proteasome. J. Bacteriol. 182, 1680-1692 (2000).

31. Benaroudj, N. \& Goldberg, A. L. PAN, the Proteasome-activating Nucleotidase from Archaebacteria, is a Protein-unfolding Molecular Chaperone. Nat. Cell Biol. 2, 833-9 (2000).

32. Braun, B. C. et al. The Base of the Proteasome Regulatory Particle Exhibits Chaperone-like Activity. Nat. Cell Biol. 1, 221-6 (1999).

33. Djuranovic, S. et al. Structure and Activity of the N-terminal Substrate Recognition Domains in Proteasomal ATPases. Mol. Cell 34, 580-590 (2009).

34. Mason, J. M. \& Arndt, K. M. Coiled Coil Domains: Stability, Specificity, and Biological Implications. Chembiochem 5, 170-6 (2004).

35. Zhang, F. et al. Structural Insights into the Regulatory Particle of the Proteasome from Methanocaldococcus jannaschii. Mol. Cell 34, 473-484 (2009).

36. Lupas, A. Coiled coils: New Structures and New Functions. Trends Biochem. Sci. 21, 375-82 (1996).

37. Kon, T. et al. Helix Sliding in the Stalk Coiled Coil of Dynein Couples ATPase and Microtubule Binding. Nat. Struct. Mol. Biol. 16, 325-33 (2009).

38. Croasdale, R. et al. An Undecided Coiled Coil: the Leucine zipper of Nek2 Kinase Exhibits Atypical Conformational Exchange Dynamics. J. Biol. Chem. 286, 27537-47 (2011).

39. Liu, Y. et al. Bicaudal-D uses a Parallel, Homodimeric Coiled Coil with Heterotypic Registry to Coordinate Recruitment of Cargos to Dynein. Genes Dev. 27, 1233-1246 (2013).

40. Macheboeuf, P. et al. Streptococcal M1 Protein Constructs a Pathological Host Fibrinogen Network. Nature 472, 64-8 (2011).

41. Parry, D. a D., Fraser, R. D. B. \& Squire, J. M. Fifty Years of Coiled-coils and Alpha-helical Bundles: A Close Relationship Between Sequence and 
Structure. J. Struct. Biol. 163, 258-69 (2008).

42. Reuter, C. J., Kaczowka, S. J. \& Maupin-furlow, J. A. Differential Regulation of the PanA and PanB Proteasome-Activating Nucleotidase and 20S Proteasomal Proteins of the Haloarchaeon Haloferax volcanii Differential Regulation of the PanA and PanB Proteasome-Activating Nucleotidase and 20 S Proteasomal Protein. J. Bacteriol. 186, 7763-7772 (2004).

43. Maupin-Furlow, J. A. Prokaryotic Ubiquitin-like Protein Modification. Annu. Rev. Microbiol. 68, 155-175 (2014).

44. Prunetti, L. et al. Structural and Biochemical Properties of an Extreme 'SaltLoving' Proteasome Activating Nucleotidase from the Archaeon Haloferax volcanii. Extremophiles 18, 283-93 (2014).

45. Bhattacharyya, S., Yu, H., Mim, C. \& Matouschek, A. Regulated Protein Turnover: Snapshots of the Proteasome in Action. Nat. Rev. Mol. Cell Biol. 15, $122-$ 133 (2014).

46. Moitra, J., Szilák, L., Krylov, D. \& Vinson, C. Leucine is the Most Stabilizing Aliphatic Amino Acid in the d Position of a Dimeric Leucine Zipper Coiled Coil. Biochemistry 36, 12567-12573 (1997).

47. Careaga, C. L. \& Falke, J. J. Structure and Dynamics of Escherichia coli Chemosensory Receptors Engineered Sulfhydryl Studies. Biophys. J. 62, 209-219 (1992).

48. Smith, D. M., Fraga, H., Reis, C., Kafri, G. \& Goldberg, A. L. ATP Binds to Proteasomal ATPases in Pairs with Distinct Functional Effects, Implying an Ordered Reaction Cycle. Cell 144, 526-38 (2011).

49. Lane, L. B. Freezing Points of Glycerol and its Aqueous Solutions. Ind. Eng. Chem. 17, 924 (1925).

50. Zhou, N. E., Kay, C. M. \& Hodges, R. S. Disulfide Bond Contribution to Protein Stability: Positional Effects of Substitution in the Hydrophobic Core of the Two-stranded Alpha-helical Coiled-coil. Biochemistry 32, 3178-3187 (1993).

51. Snoberger, A., Anderson, R. T. \& Smith, D. M. The Proteasomal ATPases Use a Slow but Highly Processive Strategy to Unfold Proteins. Front. Mol. Biosci 4, (2017).

52. Kim, Y.-C., Snoberger, A., Schupp, J. \& Smith, D. M. ATP Binding to Neighbouring Subunits and Intersubunit Allosteric Coupling Underlie Proteasomal ATPase Function. Nat. Commun. 6, 8520 (2015).

53. Peth, A., Kukushkin, N., Bossé, M. \& Goldberg, A. L. Ubiquitinated Proteins 
Activate the Proteasomal ATPases by Binding to Usp14 or Uch37 Homologs. J. Biol. Chem. 288, 7781-7790 (2013).

54. Bashore, C. et al. Ubp6 Deubiquitinase Controls Conformational Dynamics and Substrate Degradation of the 26S Proteasome. Nat. Struct. Mol. Biol. 22, 712-719 (2015).

55. Smith, D. M. et al. ATP binding to PAN or the 26S ATPases Causes Association with the 20S proteasome, Gate Opening, and Translocation of Unfolded Proteins. Mol. Cell 20, 687-98 (2005).

56. Smith, D. M. et al. Docking of the Proteasomal ATPases' Carboxyl Termini in the 20S Proteasome's Alpha Ring Opens the Gate for Substrate Entry. Mol. Cell 27, 731-44 (2007).

57. McDonnell, A., Jiang, T., Keating, A. \& Berger, B. Paircoil2: Improved Prediction of Coiled Coils from Sequence. Bioinformatics 22, 356-358 (2006). 
Chapter 5: Conclusions 


\section{Summary}

Virtually every cellular process relies on properly regulated protein degradation, and improper regulation of protein degradation leads to human disease (e.g. cancers, neurodegenerative diseases, cardiomyopathies). The proteasome is solely responsible for targeted protein degradation in eukaryotes, and the process of substrate selection and commitment by the proteasome is the most critical step in proper regulation of protein degradation. For example, the $26 \mathrm{~S}$ proteasome recognizes polyubiquitinated proteins, however, ubiquitin binding to the proteasome does not commit the substrate for degradation. In fact, after ubiquitin binding, deubiquitinase enzymes will begin to cleave off the ubiquitin molecules and the substrates are often released. To prevent premature release the substrate must bind to the proteasome's ATPases which utilize ATP to pull on the substrate and engage it, unfold it, and inject it into the activated catalytic core of the proteasome where it is degraded. Therefore, it is critical that substrate binding to the receptors efficiently activates the proteasome (by stimulating the ATPases' translocation machinery as well as by opening the gate of the $20 \mathrm{~S}$ degradation chamber). However, the receptors are separated by a great distance from the ATPase's translocation machinery and the 20S gate $(\sim 75$ and $135 \AA)$ and it is not known how such a critical message could be communicated to these distant domains. Cryo-EM structures revealed that coiled-coil (CC) domains that extend out from the ATPase subunits and form contacts with many of the non-ATPase subunits, including the ubiquitin receptors and deubiquitinases. Furthermore, substrate binding results in large conformational changes in the non-ATPase (Rpn) subunits that appear to center around these CC domains (Matyskeila et al. 2013). What wasn't known was how 
substrate binding allosterically regulates the conformational changes that modulate ATPase activity, or if structural changes in the CC domain allow for these regulatory mechanisms. We hypothesized that communication between the distant ATPase and non-ATPase subunits is allosterically mediated through specific conformational changes in the CC domains. Unfortunately, enzymological efforts using the 195 had proceeded slowly in part because of its heterogeneity and complexity. Thus, it was an attractive strategy to use a more tractable system to study the proteasomal ATPases, and then apply the principles learned to the more complex $19 S$ system. We used the archaeal ATPase, PAN, which is highly homologous to the 19S ATPase ring. We initially planned to test this hypothesis with the following 2 specific aims:

Aim 1: To determine the conformational changes that occur in the coiled-coils of the proteasomal ATPases, we will A) generate cysteine mutants in PAN that allow for disulfide crosslinking of the coiled-coil domains in various conformational states and B) use these to determine how substrate and nucleotide binding alter the range of $\mathrm{CC}$ conformational states that exist.

Aim 2: To determine the role of the coiled-coils' conformational changes in proteasome function we will restrict conformational changes using our established crosslinked mutants and employ biochemical and biophysical techniques to assess the functions of these conformational changes.

We expected these outcomes to demonstrate that conformational changes in the CC domains: 1) are fundamental to the proper functioning of the proteasomal regulatory 
ATPases, 2) are necessary conduits to communicate signals of substrate binding that trigger protein degradation, and 3) may impart flexible structural roles by both stabilizing the hexameric state of the ATPase ring while still allowing conformational changes that drive unfolding. These outcomes were expected to have a positive impact because they would provide a mechanistic framework to understand how the proteasomal ATPases regulate substrate degradation, which would inform the field how this process could be exploited in disease states.

In order to test these aims, employed a disulfide engineering approach to disrupt these conformational changes. We first designed a disulfide crosslinkable mutant that, based on the available structures of PAN's CC-OB domains, should have been in close enough proximity to form a disulfide crosslink under oxidizing conditions (the 'M87C' mutation, where the most proximal hydrophobic 'd' residue in PAN's CC, M87, was mutated to a cysteine). However, we were surprised to find that only $33 \%$ of these residues could crosslink, even when saturating levels of oxidizing agent were used (see Fig. 4.2B-C). This crosslinking happened quite quickly, as near-full-crosslinking was observed after only 5 minutes, and no additional crosslinking was observed even after 72 hours (see Fig. 4.2D). As an aside, these long incubations required protease inhibitors to prevent cleavage of PAN by low levels of contaminating proteases-and no additional crosslinking was observed at 2 weeks, even after the effectiveness of the protease inhibitors had depleted and PAN cleavage products appeared. Based on this, we hypothesized that there was some structural restraint in PAN that prevented any more than 1 out if its $3 \mathrm{CCs}$ from crosslinking. Indeed, when we relieved this structural 
restraint, either by denaturant (SDS or urea) or by removing the AAA+ ATPase domains, we observed disulfide crosslinking in all 3 of PAN's CC domains (see Fig. 4.2E, 4.3C). This indicated that structural restraints arising from the AAA+ ATPase domains must prevent any more than 1 out of PAN's 3 CC domains from crosslinking at the $\mathrm{M} 87 \mathrm{C}$ residue.

We next sought to determine what conformations PAN's other $2 \mathrm{CC}$ domains could adopt that prevent them from crosslinking at the M87C residue. To this end, I designed and purified 78 mutations to PAN's CC domains, most of which were some combination of residues mutated to cysteines. We found that PAN's 3 CC domains populate at least 3 distinct conformations: $\mathrm{C} 1, \mathrm{C} 2$, and $\mathrm{C} 3$, where $\mathrm{C} 1$ is in-register and zipped (e.g. which M87C can lock-the 33\% crosslinking observed above), C2 is inregister and partially unzipped (e.g. unzipped at the residues more proximal to the OB domain, then rezipped more distally), and C3 is slidden out-of-register by 2 heptads (see Fig. 4.4C \& 4.5D). The C2 and C3 conformations are mutually exclusive (i.e. they switch from one another), and crosslinking the C2 conformation locks a more 'active' state in PAN, while crosslinking the $\mathrm{C} 3$ conformation locks a less active state in PAN (see Fig. 4.7D)

The most stable conformation for any parallel CC domain is in-register and zipped. The fact that 2 of PAN's 3 CCs are not in the C1 conformation indicates that there are allosteric restraints in the hexameric ring that only permit one $\mathrm{C} 1$ conformation, forcing the others to adopt alternative states (i.e. C2 and C3). Thus, the C1 conformation breaks PAN's symmetry such that it is "locked" in an asymmetric state. 
Interestingly, the $\mathrm{C} 1$ conformation relies on residue $\mathrm{M} 87$, as when this residue is mutated to an alanine an increase in PAN's basal ATPase rate is observed [compare no substrate (white bars) in M87A to WT; see Fig. 4.6C]. We propose that this tends to pause cycles of ATP hydrolysis at specific pairs of subunits, thus adding a slight energy barrier to overcome each before committing to another cycle of ATP hydrolysis. Activation by the M87A mutation as well as by substrate binding seem to converge on a common mechanism, since their effect is not additive when combined together (activation by GFPssrA = activation by GFPssrA + activation by M87A; see Fig. 4.6C). We postulate that this shared mechanism is via conformational alteration of PAN's CC domains, perhaps by shifting the population of PANs with C2 versus C3 conformations.

Although the $\mathrm{CC}$ domain conformations were indeed fundamental to the proper functioning of the proteasomal ATPases (expected outcome \#1 above), conformational changes did not appear to be necessary in all of the $\mathrm{CC}$ domains, as the " $\mathrm{C} 1$ " conformation could remain in-register and zipped throughout the entire ATP hydrolysis cycle and PAN still functioned normally (e.g. see Fig. 4.6A-B- 87C oxidized), and PAN's ATPase activity even increases slightly when its $\mathrm{C} 1+\mathrm{C} 2 \mathrm{CC}$ domains are crosslinked (see Table S4.2). As an extension of this finding, although we found that conformational switching of the CC domains appear to regulate ATP hydrolysis rates, the CCs do not appear to be "communication conduits", at least not in the way that we envisioned in expected outcome \#2 above (e.g. PAN's CC domains do not function as the "on/off" switch that's been observed in other coiled-coils like dynein). Rather, we found that PAN can exist in 2 conformations: one with high activity (stabilized by crosslinking the C2 
conformation) and one with low activity (stabilized by crosslinking the C3 conformation). Since these two states are mutually exclusive (i.e. they "switch") from one another, it seems that the CC domains mainly function to impart flexible structural roles by both stabilizing the hexameric state of the ATPase ring, and that different CC conformations can either enhance or inhibit the conformational changes that drive unfolding (similar to expected outcome \#3 above).

The above data suggest that even though PAN is made up of 6 identical subunits, that it, like the heterohexameric 19S, also adopts an asymmetrical structure. We hypothesized that PAN would adopt a similar structure as the eukaryotic 19S, and also that it would bind and hydrolyze nucleotides in a similar fashion. However, at the time that we first started characterizing PAN's CC domains, there was no precedent that PAN would adopt an asymmetrical structure like the 19S does. In fact, until this point, it had been assumed that the 19S ATPases are asymmetrical in part due to their many associated non-ATPase subunits, and in part because each of the 6 subunits are different. Indeed, when we first suspected that PAN's CC domains were asymmetric, even we were surprised by this fact. It was unknown to what extent we could extend our findings to the 19S, as it was initially be difficult to imagine how 6 identical subunits could come together asymmetrically. If, indeed, PAN is asymmetrical, it was reasonable to hypothesize that this asymmetry may help to allosterically regulate ATP hydrolysis. Therefore, the overall goal of this project was extended to not only elucidating the conformations of the CC domains, but also to determine how allosteric interactions within the proteasomal ATPases catalyze protein degradation. In fact, we started this 
project very early on- soon after we first discovered that PAN's $3 \mathrm{CC}$ domains could not be crosslinked with the 'M87C' mutant (i.e. not all CCs are adopt an in-register and zipped 'C1' conformation). So, the following work was conducted concurrent with our studies on the CC domains, and was actually published prior to our full characterization of the CC domains, which we believe also helped the field to accept our asymmetrical CC conformation model for PAN.

As a first step to fulfilling our overall goal of understanding allosteric interactions within the proteasomal ATPases, we sought to understand the driving force behind all of the proteasome's actions-nucleotide binding and hydrolysis. We knew that ATP could only be bound by two of PAN's 6 ATPase subunits at any one time, and yet this was sufficient to coordinate the mechanisms that drive the translocation of proteins through its pore and into the 20S. This "paired" pattern of ATP hydrolysis appeared to be generated by both positive and negative allosteries between its subunits that control which subunits are allowed to bind ATP (e.g. see Fig. 1.2A-B). However, neither the proteasome nor the AAA+ fields understood how internal mechanisms in the ATPases control these allosteries to drive their function. We had initially hypothesized that the proteasomal ATPases would bind to opposite ("para") subunits, but this was based only on conjecture (e.g. see Fig. 1.2B). Therefore, in order to determine the binding pattern we used a nucleotide-nucleotide FRET approach to determine the distances between nucleotides. Surprisingly, we determined that both PAN and the 19S ATPases bound nucleotides on adjacent ("ortho") subunits (e.g. see Fig. 2.1E-F). Although such an ortho-binding pattern was initially surprising, we determined that such a pattern may 
make sense given that PAN contains trans-arginine fingers (i.e. arginine fingers that are contributed by the clockwise-subunits to the nucleotide binding site. Therefore, we mutated the arginine finger residues to alanines to determine their effect on nucleotide binding and hydrolysis. As expected, hydrolysis was completely inhibited (as the arginine fingers are thought to coordinate the $\mathrm{y}$-phosphate during nucleotide hydrolysis) (see Fig. 2.2A). Interestingly, although hydrolysis was completely impaired, these mutants exhibited completely normal binding (see Fig. 2.2B \& 2.3) and even maintained their 2 high and 2 low affinity binding sites, where ATP bound the high affinity sites on adjacent subunits (see Fig. 2.4). However, although this binding was normal, PAN could no longer evoke its ATP-binding effects (substrate binding and 20S gate opening, see Fig. 2.5). We thus hypothesized that the trans-functioning arginine-finger contacts ATP in its counter-clockwise neighbor, and that this must allosterically trigger substrate binding and HbYX exposure in its own subunit (arginine subunit) (see Fig. 2.7E). Indeed, following our mutagenesis study on PAN, another group solved the cryo-EM structures of another ATP-dependent protein translocase (YME1) that also contains trans-arginine fingers, and they observed what they termed an "intersubunit signaling" motif that appears to be an allosteric communication network that allows the arginine fingers to trigger substrate binding when it senses an ATP bound to its counterclockwise neighbor (Puchades et al. Science, 2017).

Since the nucleotide binding pattern was unaffected in arginine finger mutants, we reasoned that the nucleotide binding pattern must be controlled by the global structure of the ATPases. Many AAA+ ATPases, including the eukaryotic 19S 
proteasomal ATPases, adopt a "lockwasher" or "spiral staircase" conformation, and we proposed that this conformation was what regulated the ATP-binding pattern. Note that at the time, we had no way of testing which subunits were bound to ATP, since PAN itself is made up of 6 identical subunits. We will discuss in the "Future Directions" section below the strategy we developed that we believe will help circumvent this issue. However, for the purposes of building a model (e.g. see Fig. 2.7B-E), we placed the two ATP-bound subunits at the "top" subunits in the lockwasher, while the two lagging, ADPbound subunits were in the immediately clockwise subunits, and represent ATPs that have been hydrolyzed but prior to release. In order to maintain this nucleotide binding pattern over multiple rounds of ATP hydrolysis, a single subunit must hydrolyze ATP, followed by release of the lagging ADP, and then rebinding of ATP to the "new" high counter-clockwise subunit. Importantly, the arginine fingers allow for a subunit to hydrolyze ATP and move the substrate downwards without losing its 'grip' on substrate for as long as its neighboring subunit still contains an ATP. In this way, we proposed that the proteasomal ATPases maintain a tight grip on substrate throughout their ATP hydrolysis cycle, and thus must be highly processive once a substrate is engaged.

Our proposal that the proteasomal ATPases are highly processive and ordered struck some controversy in the field, as there was evidence that other ATP-dependent proteases (e.g. ClpX) are stochastic, where each of their subunits bind and hydrolyze ATP in a more-or-less independent manner. Therefore, at low levels of ATP, ClpX "slips" at more tightly folded domains of a protein (e.g. see Fig. 3.1A-possibility 1). It is important to note that ClpX does not contain trans-arginine fingers, and all of the 
conserved residues for ATPase activity are localized in one subunit. Based on this fact alone, it seemed obvious to us that ClpX likely exhibited a different mechanism of ATP hydrolysis to drive unfolding than the proteasomal ATPases. However, up until this time, we did not believe that the field appreciated the differences between proteasomal ATPases and ClpX_as each time we proposed a sequential model for PAN and the 19S, we were often cited to be incorrect on the basis of studies on ClpX. Therefore, we deemed it of high importance to clarify that although we propose a sequential mechanism of hydrolysis for the proteasomal ATPases, this does not necessarily contradict the ClpX field. Therefore, we conducted a study where we highlighted the differences between ClpX and the proteasomal ATPases (Snoberger et al. Front. Mol. Biosci., 2017). In this manuscript we presented data showing that, unlike ClpX, both the $19 S$ and PAN do not "slip" and "stall" when they reach a tightly folded domain (GFP) (e.g. see Fig. 3.2-3.3). This provided strong evidence for an ordered mechanism whereby constant grip is maintained on substrate, and we proposed that this high processivity and maintenance of grip was due to the trans-arginine fingers that sense bound nucleotides. 


\section{Future Directions}

During our extensive disulfide crosslinking analysis to determine the structure that PAN's CC domains adopt, we mutated 20 out of 28 residues in PAN's $1^{\text {st }} 4 \mathrm{CC}$ domain heptads to cysteines. We noticed that several of these mutations were capable of forming a disulfide crosslink with our model substrate (GFPssrA), both prior to translocation in an ATP-binding dependent manner (i.e. in the presence of ATPYS), as well as during translocation after unfolding (i.e. in the presence of ATP). Since the CC domains of PAN and the 19S are thought to play a crucial role in substrate binding, we propose that we can use this extensive cysteine library to generate a "substrate interaction interface" to determine residues that are important for interacting with substrate. The model substrate we use (GFPssrA) contains two cysteines, one that is solvent-exposed and another that is buried inside the beta-barrel of the protein. By selectively blocking the solvent-exposed cysteine with an irreversible thiol modifier (Nethylmaleimide), we can distinguish between the CC-substrate binding interface after substrate binding but prior to unfolding (unblocked GFPssrA), as well as the binding interface after unfolding (blocked outer cysteine, unblocked inner cysteine). Using this method, we will be able to generate a substrate-binding interface pre-unfolding and post-unfolding. In this way, we can determine which portions of the $\mathrm{CC}$ domains are responsible for substrate binding as well as chaperoning. We already have preliminary data that that provides the first direct evidence PAN's CCs interacting with substrate, and have begun to generate its full substrate interaction interface. 
Since we know that PAN's CCs adopt 3 different conformations, we wondered whether any of these 3 CCs preferentially interact with substrate. Prior to our extensive disulfide engineering approach, it would have been impossible to determine which CC interacts with substrate, since all 3 CCs are made up of identical residues. However, we hypothesize that we could use our crosslinkable $\mathrm{C} 1, \mathrm{C} 2$, and $\mathrm{C} 3$ mutants in combination with mutants that can crosslink with substrate in order to distinguish which coiled-coil (if any) preferentially interacts with substrate. Since locking the C3 conformation places PAN in an inactive conformation, we plan to focus on the mutations that can crosslink C1 and $\mathrm{C} 1+\mathrm{C} 2$ : $\mathrm{PAN}-87 \mathrm{C}$ and $\mathrm{PAN}-59 \mathrm{C}$, respectively (which lock fully functional versions of PAN). The disulfide engineering approach we plan to use is quite unique, since even though each of PAN's 6 subunits are identical, this approach allows us to identify PAN's subunits based on the conformations they adopt (e.g. uncrosslinked monomers and disulfide crosslinked dimers on non-reducing SDS-PAGE). So, we have combined 7 of our substrate-crosslinkable mutations with these $\mathrm{C} 1$ and $\mathrm{C} 1+\mathrm{C} 2$ PAN mutants (14 mutants total), and will to ask whether substrate preferentially interacts with any of these 3 CCs (i.e. we will ask whether GFPssrA preferentially formed disulfide bonds with either PAN monomers or dimers in these specific mutants, or whether there was no preference). In Figure $\mathbf{5 . 1}$ we have laid out the logic for determining the CC responsible for binding substrate given for each possible result.

We have tested 4 of these 14 mutants, and preliminary data suggest that GFPssrA interacts exclusively with dimers in both the $\mathrm{C} 1$ and $\mathrm{C} 1+\mathrm{C} 2$ crosslinkable variants during unfolding. Based on Figure 5.1, this indicates that GFPssrA binds only 


\begin{tabular}{|c|c|c|}
\hline \multirow[b]{2}{*}{ Conformation } & \multicolumn{2}{|c|}{ Which $\mathrm{CC}$ dimers interact with substrates } \\
\hline & (C1 $\frac{87 \mathrm{C}}{\text { crosslink) }}$ & $\left(\mathrm{C} 1+\mathrm{C} \frac{\mathbf{5 9 C}}{2 \text { crosslink })}\right.$ \\
\hline C1 & 100\% Dimer & 100\% Dimer \\
\hline C2 & 0\% Dimer & 100\% Dimer \\
\hline C3 & 0\% Dimer & 0\% Dimer \\
\hline C1 \& C2 & $50 \%$ Dimer & 100\% Dimer \\
\hline C1 \& C3 & $50 \%$ Dimer & $50 \%$ Dimer \\
\hline C2 \& C3 & 0\% Dimer & $50 \%$ Dimer \\
\hline $\begin{array}{l}\text { No Preference } \\
(C 1+C 2+C 3)\end{array}$ & 33\% Dimer & $67 \%$ Dimer \\
\hline
\end{tabular}

Figure 5.1: General logic to determine which subunits bind to nucleotides, substrates, or the $20 \mathrm{~S}$ proteasome. This is a general logic table to determine which subunits are responsible for the various functions of PAN, including chaperoning, nucleotide binding, substrate engagement, and $20 \mathrm{~S}$ engagement. All experiments utilize the $87 \mathrm{C}$ and $59 \mathrm{C}$ crosslinkable mutants, which dimerize the $\mathrm{C} 1$ or $\mathrm{C} 1+\mathrm{C} 2$ coiled-coils, respectively. The red box indicates the results of our preliminary data on PAN's chaperone activity, where a mutant that forms a disulfide crosslink with GFPssrA following unfolding (e.g. PAN-86C) was combined with either the 87C or 59C mutations. One can deduce which subunit is bound to substrate by quantifying the percentage of substrate bound to monomers vs. dimers (\% dimers are shown above for simplicity). Assays to test nucleotide binding, substrate engagement, and 20S engagement use similar logic as outlined in this table, and are described in more detail in Figure 5.2. 
to the $\mathrm{C} 1 \mathrm{CC}$ during unfolding. We believe that this unfolding-dependent binding occurs during PAN's "chaperoning" of substrates. Indeed, up until the time that we started studying PAN's CC domains, such prevention of aggregation ("chaperone") activity was the only function that they had been definitively attributed to these CC domains in vitro. One study reported that PAN's CC domains were quite flexible (Zhang et al. Mol Cell 2009), and at the time it was presumed that this flexibility played a large role in chaperoning. Also based on this flexibility, it was expected that all $3 \mathrm{CCs}$ played a role in chaperoning, where they could flexibly and reversibly "take turns" binding and releasing substrate during unfolding. However, our preliminary data suggest that only PAN's C1 CC domain plays a role in unfolding-dependent binding, and since we have already demonstrated that this CC remains in-register and zipped throughout PAN's ATP hydrolysis cycle (discussed in detail in Chapter 4), we hypothesize that stabilizing the C1 CC domains via disulfide crosslink should actually enhance (not decrease) chaperone activity. Therefore, to test this hypothesis, we propose to conduct similar chaperoning experiments as in Benaroudj \& Goldberg, 2000 using our C1 and C1+C2 crosslinkable mutants.

Inspired by our promising preliminary studies on the substrate binding interface on PAN, we propose that we can learn more about PAN's asymmetries using these crosslinkable CC mutations. As was alluded to earlier in this discussion's summary section, we know that PAN has two high-affinity ATP binding sites that are located on adjacent subunits, but we are unsure which (if any) subunits bind first to ATP. Previously, this would have been a nearly impossible question to answer, since PAN is 
made up of 6 identical subunits. In our discussion in Chapter 4 , we envisioned that PAN's CC domains helped to "set" PAN to allow for initial ATP binding, and then hydrolysis occurs around the ring, pausing once it's reached a full $360^{\circ}$ round of ATP hydrolysis. Therefore, we propose that if one could find a way to irreversibly modify subunits upon nucleotide binding, that using our crosslinkable $\mathrm{C} 1$ and $\mathrm{C} 1+\mathrm{C} 2$ mutants, we should be able to determine which of these subunits initially bind to nucleotide using a similar logic strategy that we used to determine the subunit binding interface. Indeed, we have found two such modifiers of ATPase subunits, "desthiobiotin-ATP" and "desthiobiotin-ADP" (Sigma), which irreversibly modify active site lysines (e.g. Walker A lysines) with a desthiobiotin group following nucleotide binding. Using this probe we can ask which subunits have been modified with a desthiobiotin via western blotting (e.g. we can ask whether biotin modifies monomers or dimers in specific subunits, using similar logic as in Figure 5.1). We currently have preliminary data to show that these probes bind to PAN (WT PAN, PAN-C1, and PAN-C1+C2) in the presence, but not absence of magnesium, indicating its specificity for PAN's ATP binding sites. Future experiments will test whether this probe binds to monomers or dimers in the $\mathrm{C} 1$ or $\mathrm{C} 1+\mathrm{C} 2$ mutations, and in this manner, we can determine which two subunits have high affinity for ATP using the logic in Figure 5.1. Furthermore, assuming desthiobiotin modification of the active site doesn't alter the nucleotide binding pattern, we also hypothesize that we will be able to determine the low affinity nucleotide binding sites by using increasing amounts of dethiobiotin-ATP, or by utilizing nonhydrolyzable ATPYS to occupy the two high affinity sites, then adding desthiobiotin-ADP. 
Notably, we do not yet know in what order the CCs oligomerize (e.g. C1-C2-C3 or $\mathrm{C} 1-\mathrm{C} 3-\mathrm{C} 2$, Fig. 5.2A). In Chapter 4, we proposed a $\mathrm{C} 1-\mathrm{C} 3-\mathrm{C} 2$ conformation based on a cryo-EM structure of the 19S proteasomal ATPases, but note that although the reported resolution of this structure is $\sim 6 \AA$, based on the reported b-factors we estimate the resolution at the CC domains to be $\sim 20 \AA$. With the above desthiobiotin-ATP results, we believe that we will narrow down the ATP-binding pattern to a few possibilities, but further experimentation would be required to differentiate between these models. We expect that the ADP-bound subunits will occupy the two clockwise-subunits to the two ATP-bound subunits. In Figure 5.2A-B, we provide an example of how we can use data from the desthiobiotin experiments to determine the order in which the CCs oligomerize. In Figure 5.1A, we have hypothetically placed the high affinity ATP-bound subunits at the two subunits at the interface of $\mathrm{C} 1 / \mathrm{C} 2$ (red circles). Based on similar logic as in Figure 5.1, we show the result one would expect if ATP binds at the interface of $\mathrm{C} 1$ and C2, for both possible PAN topologies: $50 \%$ of biotin would crosslink to dimers in the $\mathrm{C} 1$ mutant, and $100 \%$ would crosslink to dimers in the $\mathrm{C} 1+\mathrm{C} 2$ mutant in both the $\mathrm{C} 1-\mathrm{C} 2-\mathrm{C} 3$ and C1-C3-C2 PAN topologies (Fig. 5.2B, red text). We expect the two low affinity ADP sites to be in the subunits directly clockwise of the high affinity sites, which indicates that if PAN is in the $\mathrm{C} 1-\mathrm{C} 2-\mathrm{C} 3$ conformation, one would expect the low affinity nucleotide sites (blue circles) should occupy the subunits at the interface of C2/C3, whereas if PAN is in the $\mathrm{C} 1-\mathrm{C} 3-\mathrm{C} 2$ conformation, one would expect these low affinity sites to occupy the subunits at the C1/C3 interface (Fig. 5.2A-B; blue circles). 

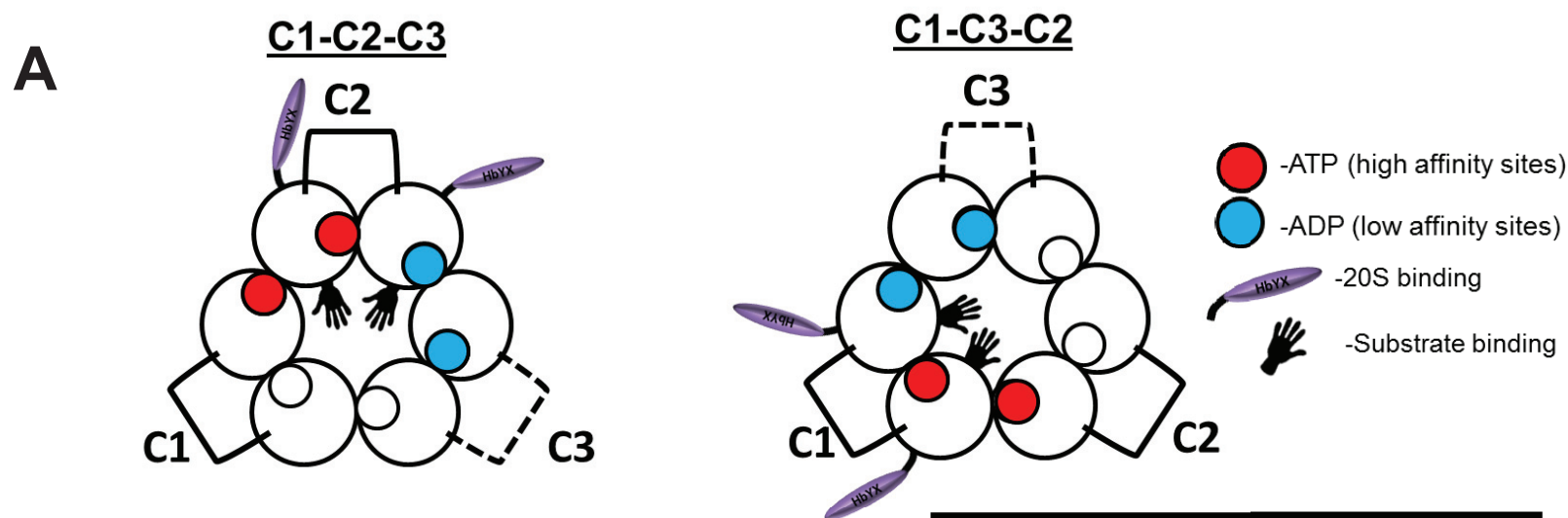

\begin{tabular}{|c|c|c|c|c|c|c|c|}
\hline \\
\hline$\frac{\text { PAN function }}{\text { tested }}$ & Substrate Used & $\frac{\text { PAN }}{\text { modification }}$ & 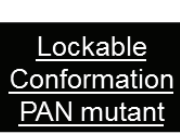 & $\begin{array}{r}\underline{\mathrm{C} 1-} \\
\text { \% dimers } \\
\end{array}$ & $\begin{array}{l}\text { 2-C3 } \\
\text { Bound } \\
\text { subunit(s) } \\
\end{array}$ & $\begin{array}{r}\text { C1- } \\
\text { \% dimers } \\
\end{array}$ & $\begin{array}{l}\text { 3-C2 } \\
\text { Bound } \\
\text { subunit(s) } \\
\end{array}$ \\
\hline \multirow{2}{*}{$\begin{array}{l}\text { ATP (high } \\
\text { affinity) }\end{array}$} & \multirow{2}{*}{ db-ATP } & \multirow{2}{*}{$\begin{array}{l}\text { None (except } \\
\text { C1 or C1+C2) }\end{array}$} & C1 & $\begin{array}{c}50 \% \\
\text { dimers }\end{array}$ & \multirow{2}{*}{$\mathrm{C} 1+\mathrm{C} 2$} & $\begin{array}{c}50 \% \\
\text { Dimers }\end{array}$ & \multirow{2}{*}{$\mathrm{C} 1+\mathrm{C} 2$} \\
\hline & & & $\mathrm{C} 1+\mathrm{C} 2$ & $\begin{array}{c}100 \% \\
\text { dimers }\end{array}$ & & $\begin{array}{c}100 \% \\
\text { dimers }\end{array}$ & \\
\hline \multirow{2}{*}{$\begin{array}{l}\text { ADP (low } \\
\text { affinity) }\end{array}$} & \multirow{2}{*}{$\begin{array}{l}\text { db-ATP or } \\
\text { db-ADP }\end{array}$} & \multirow{2}{*}{$\begin{array}{l}\text { None (except } \\
\text { C1 or } \mathrm{C} 1+\mathrm{C} 2)\end{array}$} & C1 & $0 \%$ & \multirow{2}{*}{$\mathrm{C} 2+\mathrm{C} 3$} & $50 \%$ & \multirow{2}{*}{$\mathrm{C} 1+\mathrm{C} 3$} \\
\hline & & & $\mathrm{C} 1+\mathrm{C} 2$ & $50 \%$ & & $50 \%$ & \\
\hline \multirow{2}{*}{$\begin{array}{c}\text { Substrate } \\
\text { Engagement }\end{array}$} & \multirow{2}{*}{$\begin{array}{c}\text { GFP- } \\
\text { N6CssrA }\end{array}$} & \multirow{2}{*}{$\begin{array}{l}\text { Pore loop } \\
\text { cysteine }\end{array}$} & $\mathrm{C} 1$ & $100 \%$ & \multirow{2}{*}{$\mathrm{C} 2$} & $100 \%$ & \multirow{2}{*}{ C1 } \\
\hline & & & $\mathrm{C} 1+\mathrm{C} 2$ & $100 \%$ & & $0 \%$ & \\
\hline \multirow{2}{*}{ 20S Binding } & \multirow{2}{*}{ 20S-K66C } & \multirow{2}{*}{$\begin{array}{c}\text { C-terminal } \\
\text { HbYX } \\
\text { cysteine }\end{array}$} & C1 & $100 \%$ & \multirow{2}{*}{$\mathrm{C} 2$} & $100 \%$ & \multirow{2}{*}{ C1 } \\
\hline & & & $\mathrm{C} 1+\mathrm{C} 2$ & $100 \%$ & & $0 \%$ & \\
\hline
\end{tabular}

Figure 5.2: Determining the topology that the PAN hexamer adopts. A) 2 possibilities for PAN topology are C1-C2-C3, and C1-C3-C2. B-left half of table) Substrate and PAN variants used to test PAN functions: ATP/ADP binding, substrate engagement, and $20 \mathrm{~S}$ binding. The $87 \mathrm{C}$ and $59 \mathrm{C}$ mutants can be used alone to determine nucleotide binding sites by using desthiobiotin(db)-ATP or db-ADP, which irreversibly modify active site lysines with a desthiobiotin group. The $87 \mathrm{C} \& 59 \mathrm{C}$ mutants can also be combined with 2 other types of PAN cysteine mutants: 1) PAN pore-loop cysteine mutants that allow for crosslinking to a GFPssrA with a cysteine on its ssrA tag (GFP-N6CssrA), or 3) PAN with a cysteine on its C-terminus (the end of its $\mathrm{HbYX}$ motif), which can crosslink to a 20S proteasome with a cysteine on its binding pocket into which PAN's C-terminus inserts. In $\mathbf{A}$ and the right half of $\mathbf{B}$, as an example of how to distinguish between $\mathrm{C} 1-\mathrm{C} 2-\mathrm{C} 3$ vs. $\mathrm{C} 1-\mathrm{C} 3-\mathrm{C} 2$ topologies, we have laid out a hypothetical experiment where high affinity ATP sites bind at the interface of C1 and C2 (red text). It is expected that ADP will bind to the two lagging subunits clockwise to ATPbound sites, while substrate and $20 \mathrm{~S}$ engagement will occur in the immediate clockwise subunits to the ATP-bound subunits. The table in B uses hypothetical quantifications of \% dimers (using similar logic as in Figure 5.1) in order to determine which subunits are bound to nucleotide, substrate, or 20 S. 
We believe that the above experiment alone could theoretically show the topology that PAN adopts. However, in all likelihood, these experiments will not turn out as "clean" as we have laid out here. For example, we do not know to what extent binding of desthiobiotin-ATP will affect the binding of subsequent nucleotides. Therefore, we may need to utilize combinations of ATP or nonhydrolyzable ATPYS with the desthiobiotin-nucleotides in order circumvent this issue. Of note, in our preliminary data, PAN does retain its quaternary structure following desthiobiotin modification as observed by Native-PAGE, so we are hopeful that the general nucleotide binding pattern will be similar following modification with desthiobiotin. Another potential issue we may encounter with PAN's high affinity ATP sites. These experiments will require saturating PAN and very low amounts of desthiobiotin-ATP in order to see binding to only the high affinity sites. Therefore, most of the PAN population is expected to unbound, while the PAN that are bound will likely be mostly bound to only 1 nucleotide, with a minor subset of them binding 2 nucleotides. We have insufficient evidence to determine whether both of these high affinity sites have equal affinity for ATP, or whether one of these sites binds to ATP prior to the second site binding ATP. In stopped flow experiments where we compete off fluorescently-labeled nucleotides from the high affinity sites with unlabeled ADP, we only observed a $\sim 50 \%$ drop in fluorescence when we subtracted the basal fluorescence of free mant-nucleotides (see Fig. 2.6A\&C). So, it seems as though one of the two fluorescent nucleotides may remain tightly bound, and it's therefore likely that these two high affinity ATP binding sites have different ATP binding kinetics. Due to the nature of these experiments, the results may not be as straightforward to interpret as in Figure 5.1, and will likely require 
additional experiments to gain a full understanding of the nucleotide binding sites. So, it would be desirable if the ring topology could be confirmed by another method.

Based on our discussion of the arginine fingers in Chapter 2, we hypothesized that the clockwise-subunits of ATP-bound subunits engage with substrate and open the gate of the 20S (Fig. 5.2A, pore loop 'hands' and purple HbYX motifs, respectively). We therefore wondered whether we could develop a strategy with our $\mathrm{C} 1$ and $\mathrm{C} 1+\mathrm{C} 2$ crosslinkable mutations to determine which subunits are bound to substrate, and perhaps which subunits were bound to the 20S. In order to test this, in future experiments we will engineer cysteine mutations that can crosslink the GFPssrA substrate to the pore loops on PAN (which engage substrate). We have already engineered a cysteine mutation onto the $6^{\text {th }}$ residue of the ssrA tag, which we call "GFPS6CssrA", and determined in preliminary data that this GFP variant can be engaged and unfolded by WT PAN. We also plan to engineer cysteine mutations to PAN's pore loops. The pore loops of protein-translocating proteins are made up of an aromatichydrophobic-glycine motif (in PAN the sequence is "FIG"). We propose to mutate each of these residues to cysteines and determine which of these three mutations retains its capacity to bind to substrate, then will move forward to combine that mutant with our $\mathrm{C} 1$ and $\mathrm{C} 1+\mathrm{C} 2$ crosslinkable mutants. Since an isoleucine to cysteine mutation is a conserved one, we hypothesize that this mutant is the most likely candidate to retain its ATP-dependent substrate binding, but this will need to be confirmed experimentally. We propose that using this pore loop-substrate crosslinking assay, that we will be able to determine where initial substrate binding occurs based on whether substrate crosslinks 
with monomers or dimers in the $\mathrm{C} 1$ or $\mathrm{C} 2$ mutations. In our hypothetical example, one would expect one of two possibilities: if PAN adopts the C1-C2-C3 PAN topology, substrate will crosslink to $\mathrm{C} 2$ alone, but if PAN adopts the C1-C3-C2 topology substrate will bind to C1 alone (e.g. see Fig. 5.2A-B; pore loop "hands").

Future experiments will also test the other ATP-binding effect-20S bindingwhich we expect to be the same subunits that engage substrate (e.g. see the purple HbYX Motif in Fig. 5.2A-B, compared to the pore loop "hands"). We plan to test this binding by engineering a cysteine on the $20 \mathrm{~S} \mathrm{HbYX}$ binding pocket $(\mathrm{K} 66 \mathrm{C})$ as well as on the ' $\mathrm{X}$ ' residue in PAN's C-terminal HbYX motif. We expect that these residues will form disulfide crosslinks with one another, and using similar experiments and logic that we used to determine substrate binding and ATP-binding, we expect to obtain similar results as with the substrate-pore loop crosslinking assays (see Fig 5.2A-B).

Future experiments will also aim to determine whether ATP is hydrolyzed cyclically around the ring, or whether only a subset of subunits bind and hydrolyze ATP (which we discussed in detail in the Chapter 4 discussion). We favored the former explanation, but we could not rule out the latter. Using our disulfide crosslinkable mutants and varying mixtures of ATP and destihobiotin-ATP, we hypothesize that we will be able to capture PAN in the middle of its ATP hydrolysis cycle, rather than only in its basal binding state (which was the basis of Fig. 5.2). This novel technique should distinguish between binding to only a subset of subunits vs. cyclical hydrolysis. 
Lastly, we believe that our crosslinkable mutations will aid in the structural determination of PAN via electron microscopy. In EM, averaging is often used to gain high-resolution structures. However, in a homohexamer like PAN, it is often difficult to differentiate between subunits, and thus it can be difficult to align them properly. In future experiments, we will modify our exposed cysteine residues with electron-dense particles (e.g. with gold-maleimide) in order to determine the orientation of particles. For example, we propose that we could use the $C 1+C 2$ crosslinkable mutant (59C), precrosslink it, then add gold maleimide. In this experiment, gold would only modify the $\mathrm{C} 3$ conformations, since in this mutant, only the C3 CC has exposed thiols. Using this technique we would be able to definitively differentiate between subunits and properly align the particles, yielding invaluable structural information for PAN. Additionally, we believe that using this technique we will be able to obtain a high-resolution structure of PAN, and we would likely even be able to capture different nucleotide-hydrolyzing states (e.g. determining whether or not ATP is hydrolyzed around the ring, and if so, how the conformations in the AAA+ ATPase domains are linked to the CC conformations). 


\section{Impact}

The techniques we've developed here have focused on the proteasome, the cell's main degradation machinery, and we have used highly novel methods to better understand the inner workings of this complex multimeric machine. These studies have contributed greatly to our knowledge of the proteasome, and the hope is that a better understanding of this complex machine will aid in the development of new therapies to combat diseases in which the proteasome is dysregulated.

We would be remiss, however, if we focused only on how this research will impact the proteasome field. This technique we've developed can capture and distinguish between different subunits in a protein that are identical, but that adopt asymmetric intermediate structures during their functionings. Indeed, a multitude of multimeric motor proteins use energy from nucleotide hydrolysis to carry out virtually every process that requires mechanical work within the cell, and many of these proteins are homomeric. In addition to protein degradation (which we've studied here), things such as ATP synthesis, cargo transport, DNA unwinding, and cell motility utilize a variety of molecular motors. Many of these multimeric proteins are closely related to one another and may have even evolved from the same evolutionary precursor proteins. However, many of these proteins have drastically different modes of operation that allow for them to perform many different functions. Therefore, it is critical to understand the underlying mechanisms that drive these motors, but up until this point, it has been difficult to study the detailed molecular mechanisms of these proteins, in part due to the inherent structural and mechanistic heterogeneities present in these complex motors. 
Many of these motors are made up of identical subunits that adopt different conformations, but a complete understanding of these conformations has remained elusive.

There are many techniques researchers can utilize to understand the underlying mechanisms of these molecular motors, but as powerful as many techniques are, it's important that one understand each technique's advantages as well as its limitations. For example, structural data is invaluable in understanding molecular machines, however, many of these techniques are limited in the size of macromolecular complexes they can probe (e.g. nuclear magnetic resonance), and often these methods provide only snapshots of macromolecular complexes in action (e.g. X-ray crystallography, electron microscopy). Furthermore, the conditions under which structures are determined are often unnatural (high concentrations, inorganic solvents, unnatural $\mathrm{pH}$, etc.), and more mobile protein domains-which often serve important regulatory functions—often must be cleaved in order for high resolution structural information to be elucidated. Indeed, although we found the CC-OB crystal structures of PAN to be incredibly useful for designing our disulfide engineering approach in PAN's CCs, in order to generate crystals, these structures completely lacked their crucial AAA+ ATPase domains. Therefore, PAN's CC-OB domain appeared to be symmetrical in the crystal structure, with all 3 of its CCs forming in-register and zipped coiled-coils, but when we used full-length PAN to generate structural information using our disulfide crosslinking strategy, we discovered that the presence of these AAA+ ATPase domains were critical in determining what conformations the $\mathrm{CC}$ domains adopt. The newly 
emerging field of cryo-electron microscopy can literally "freeze" these motors under more "natural" conditions, however, tens or hundreds of thousands of molecules must be averaged to generate high-resolution picture. Additionally, symmetry is often imposed on these particles, and these observed averages, combined with imposing symmetry can result in inaccurate depictions of, for example, the number of nucleotides bound and the pattern of their hydrolysis. In the case of proteasomal ATPases, although from biochemical data we know that only 2 ATPs bind at a time on adjacent subunits, in cryo-EM reconstructions, weak densities can be found in every nucleotide binding pocket, likely a byproduct of such averaging.

One exciting application of our disulfide crosslinking approach is the possibility of using it in conjunction with emerging cryo-EM techniques. At present, computer programs are often used to impose symmetry and determine the orientation of particles, but such programs simply utilize a best guess of particles that resemble one another and can miss the minutia of conformational changes, especially when relying on many thousands of particles to generate a decent resolution snapshot of a protein, and such averaging is likely to miss the smaller conformational differences and less frequently populated conformational states. One striking example of this is in verbal correspondence with a researcher working on solving the structure of PAN via cryo-EM. In preliminary structures where they used an averaging technique, they found an odd "zig-zag" conformation of PAN, where the subunits were "high-low-high-low-high-low". We believe, based on the structures of the 19S ATPases as well as many other AAA+ ATPases, that PAN is in fact a "spiral staircase" conformation, but due to the imposed 
symmetry and the inability to distinguish between subunits, this odd architecture was deemed a byproduct of cryo-EM averaging, and not the actual structure that PAN adopts. In the future directions section above, we envision a method whereby our crosslinkable mutants could be used to definitively differentiate between subunits and properly align the particles. We are excited at the prospect of using this technique to study not only PAN, but to see the broad impact such a technique will have on the multimeric motor protein field in general.

In conclusion, although much can be learned from individual techniques, it is crucial to combine various techniques (e.g. structural, single molecule, biochemical, and biophysical techniques) in order to generate the most accurate picture possible of how molecular motors function in their native environments. We envision that similar disulfide engineering approaches that we've used in PAN could be new tool in this molecular tool-belt for scientists that will propel forward our understanding of molecular underpinnings of other multimeric motor proteins whose detailed mechanisms have thus far eluded us. This exciting disulfide engineering approach we've developed is a powerful method to analyze structural asymmetries in homomeric protein complexes with minimal structural perturbations, which has not been accomplished before and opens the door to an entire new approach to studying the function of the molecular motors. 


\section{References}

Benaroudj, N. \& Goldberg, A. L. PAN, the Proteasome-activating Nucleotidase from Archaebacteria, is a Protein-unfolding Molecular Chaperone. Nat. Cell Biol. 2, 833-9 (2000).

Puchades, C., Rampello, A. J., Shin, M., Giuliano C. J., Wiseman, R. L., Glynn, S.E., Lander, G.C. Structure of the mitochondrial inner membrane AAA+ protease YME1 gives insight into substrate processing. Science. Nov 3, 358 (2017).

Matyskiela, M. E., Lander, G. C. \& Martin, A. Conformational Switching of the 26S Proteasome Enables Substrate Degradation. Nat. Struct. Mol. Biol. 20, 781-8 (2013).

Zhang, F., Hu, M., Tian, G., Zhang, P., Finley, D., Jeffrey, P. D., et al. Structural Insights into the Regulatory Particle of the Proteasome from Methanocaldococcus jannaschii. Mol. Cell 34, 473-484 (2009). 